\title{
INTEGRATIONSPROJEKTE ALS AUFGABE URBANER FORSTWIRTSCHAFT
}

\section{- EIN BEITRAG ZUR SOZIALEN NACHHALTIGKEIT -}

\author{
Dissertation \\ zur Erlangung des Doktorgrades \\ der Fakultät für Forstwissenschaften und Waldökologie \\ der Georg-August-Universität Göttingen \\ vorgelegt von \\ Katharina Vering \\ geboren in Rheda-Wiedenbrück / Nordrhein-Westfalen
}


D7

1. Berichterstatter:

2. Berichterstatter:

Tag der mündlichen Prüfung:
Prof. Dr. Max Krott

Prof. Dr. Bernhard Möhring

19. September 2007

http://resolver.sub.uni-goettingen.de/purl/?webdoc-1722 


\section{Danksagung}

Die vorliegende Arbeit ist am Lehrstuhl für Forstpolitik, Forstgeschichte und Naturschutz der GeorgAugust-Universität Göttingen unter der Leitung von Prof. Dr. Max Krott entstanden. Den Grundstein für das Thema „Integrationsprojekte“ hat ein Vortrag gelegt, der „Backpacker“ als Randgruppen bezeichnete, denen der Wald eine Möglichkeit zur Unterkunft bieten könnte. Mein Interesse war geweckt, denn ich wollte herausfinden, ob Wald und somit Forstverwaltungen in der Lage sind, Integration zu fördern und ob „Backpacker“ wirklich zu einer Randgruppe gehören.

Prof. Dr. Max Krott gilt mein herzlichster Dank für das Einlassen auf ein solch spezielles Thema. Die qualifizierte Betreuung, die Gespräche und Diskussionen im Verlauf des Forschungsprozesses haben den entscheidenden Beitrag zur Fertigstellung der Arbeit geleistet. Besonders die motivierende Worte zum Ende der Fertigstellung haben die Arbeit wesentlich unterstützt.

Bei Prof. Dr. Bernhard Möhring möchte ich mich herzlich bedanken, da er sich bereit erklärt hat, die Arbeit als Zweitgutachter zu beurteilen und sich auf dieses Thema eingelassen hat.

Mein besonderer Dank gilt den Gutachtern der Georg-Ludwig-Hartig-Stiftung, die den Beitrag zur sozialen Nachhaltigkeit als fördernswert empfanden und das Forschungsprojekt mit Sach- und Reisemitteln unterstützt haben. Ohne die finanzielle Hilfe wäre das Projekt nicht umsetzbar gewesen.

Den Kolleginnen und Kollegen am Institut für Forstpolitik möchte ich ganz herzlich für vertrauensvolle und erfolgreiche Zusammenarbeit in all den Jahren danken. Die fachlichen Diskussionen in den Doktorandenkolloquien haben einen entscheidenden Beitrag zur Qualität der Arbeit geleistet. Die Zeit am Institut möchte ich nicht missen. Aus dieser Zeit sind für mich ganz wertvolle Kontakte und Freundschaften entstanden. Dr. Daniela Kleinschmit und Alejandra Real gilt mein ganz besonderer Dank, da sie mich nicht nur fachlich, sondern auch durch ihre Freundschaft unterstützen. Dr. Alexander Bittner möchte ich ganz herzlich für die „Grundsteinlegung“ dieser Arbeit danken.

Meinem „sozialen Umfeld“ gilt ein großer Dank, da es mir in der stressigen Zeit nicht mit „Desintegration" gedroht, sondern Verständnis aufgebracht hat und immer wieder motivierende Worte gefunden hat. Hier gilt mein Dank besonders Markus Euring, der mir eine unglaubliche Stütze ist.

Von ganzem Herzen möchte ich mich bei meiner Familie bedanken, ohne deren gemeinsame Unterstützung ich meinen Weg nicht hätte gehen können. Meinen Eltern und Schwestern bin ich für die vielen motivierenden und ehrlichen Gespräche während der letzten Jahre unendlich dankbar. Sie haben einen großen Anteil an dieser Arbeit. Meinen bisherigen Lebensweg haben vor allem meine Großmütter und Hanne geprägt - Ihnen ist diese Arbeit gewidmet. 


\section{INHALTSVERZEICHNIS}

\section{Abbildungsverzeichnis II Tabellenverzeichnis}

\section{EINLEITUNG}

1.1 Bedeutung von urbanen Grün- und Waldflächen .................................... 10

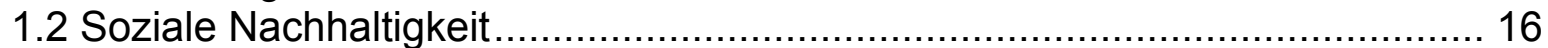

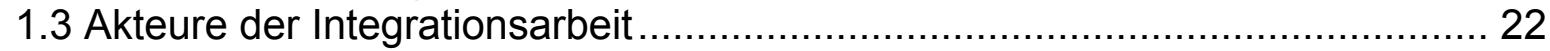

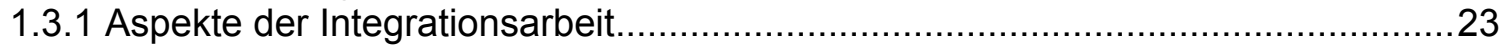

1.3.1.1 Soziale Dienste .................................................................................... 24

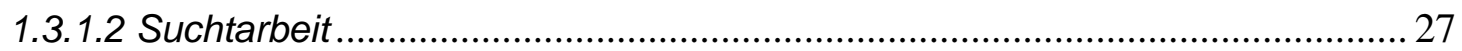

1.3.1.3 Wohnungslosenhilfe .................................................................................. 29

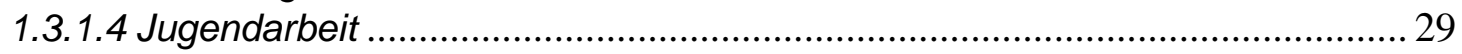

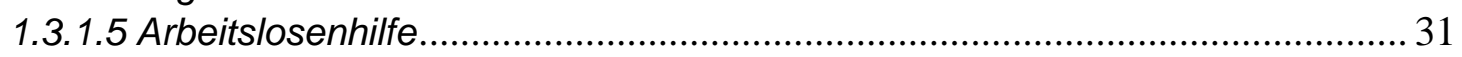

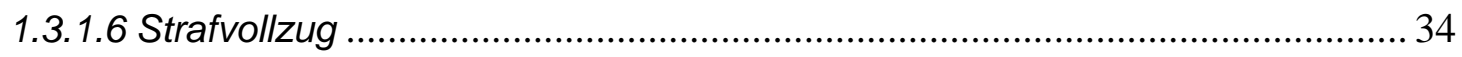

2. FORSCHUNGSANSATZ

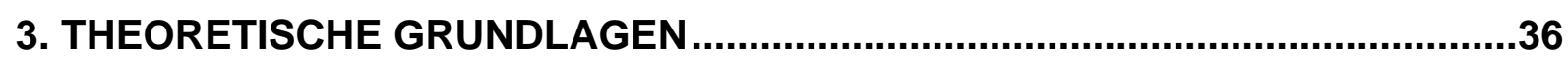

3.1 Der Randgruppenbegriff und soziale Ungleichheit ................................... 36

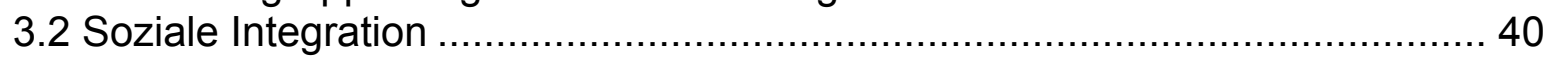

3.2.1. Liberales Modell der Sozialen Integration ......................................................4 4

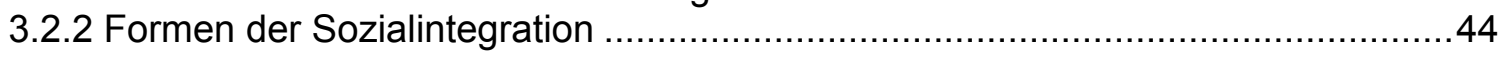

3.2.2.1 Exkurs: Potential des Waldes für die soziale Integration....................................47

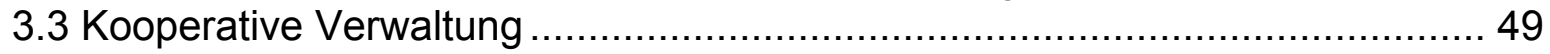

3.3.1 Merkmale kooperativen Verwaltungshandelns .............................................. 49

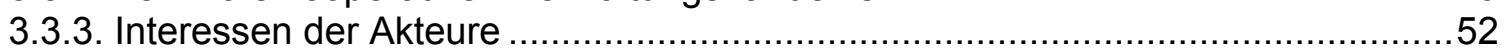

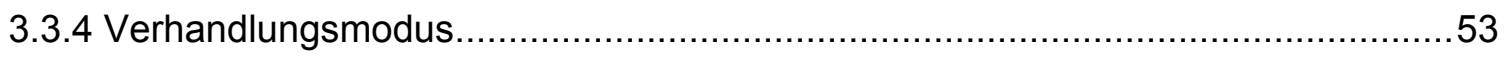

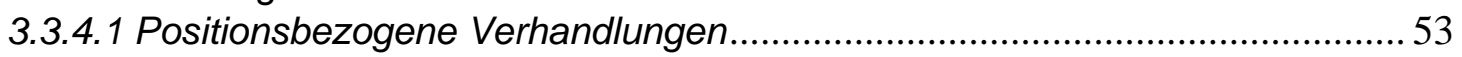

3.3.4.2 Kompromissorientierte Verhandlungen ........................................................... 54

3.3.4.3 Verständigungsorientierte Verhandlungen ...................................................... 54

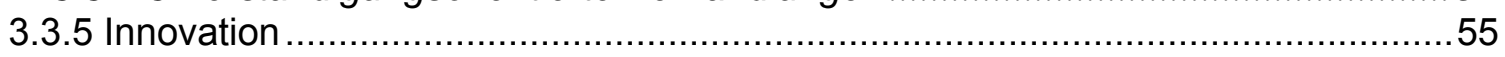

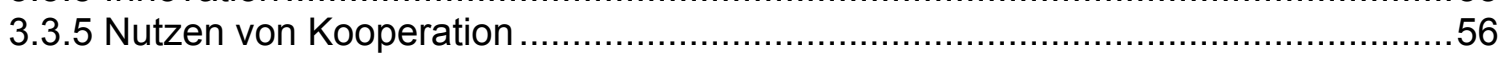

3.4 Aufgabenwahrnehmung der Forstverwaltung ……................................ 58

3.5 Neue Produkte der Forstwirtschaft....................................................... 58

3.6 Forschungsansatz und Hypothesenformulierung ...................................... 61

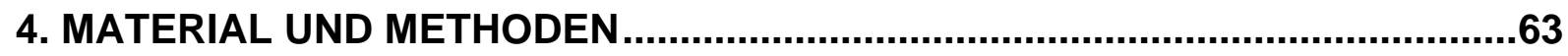

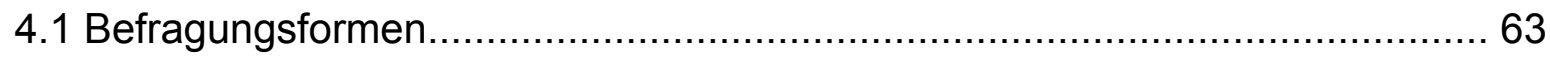

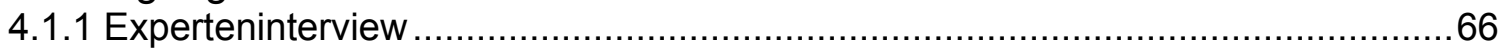

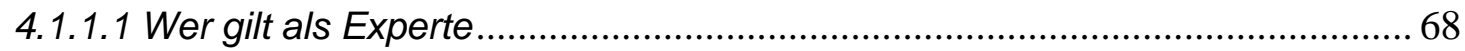

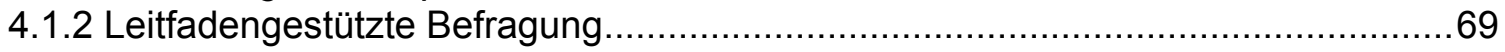

4.1.3 Datenerfassung bzw. Datenaufzeichnung ....................................................

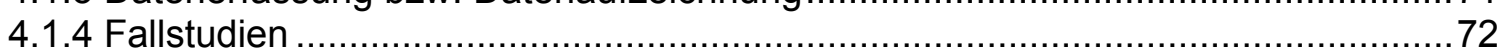

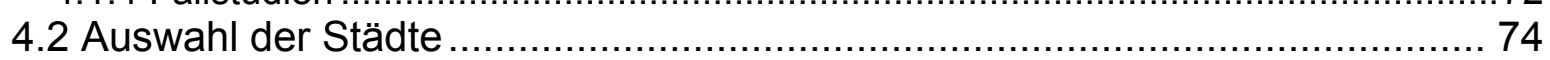

4.3 Erstellung des Fragenbogens und Kategoriensystems .............................. 76

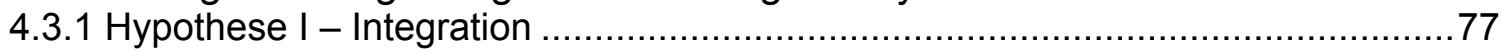

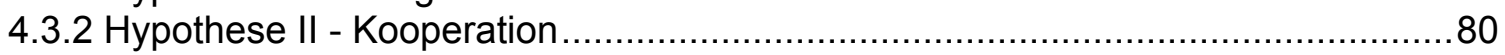

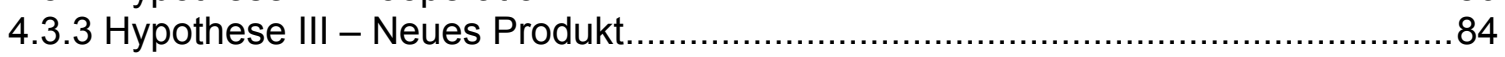




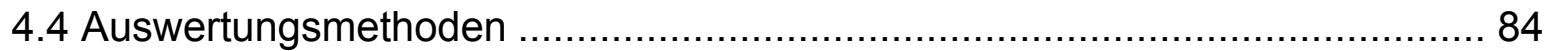

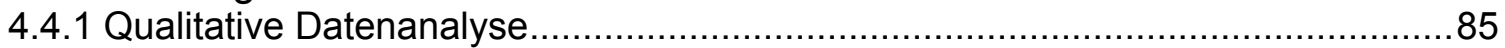

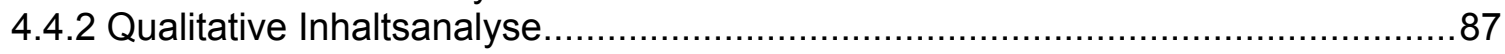

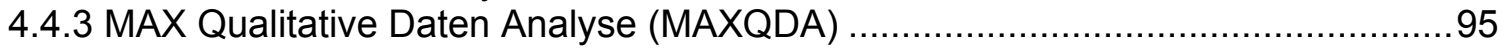

5. ERGEBNISSE UND ERGEBNISDISKUSSION ..............................................98

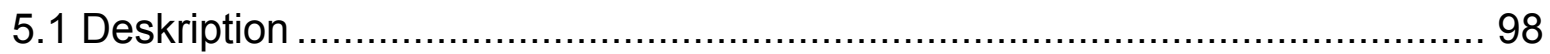

5.1.1 Berührungspunkte der Institutionen mit Randgruppen .....................................98

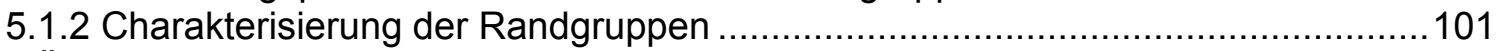

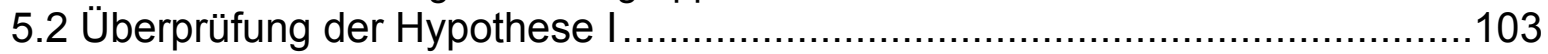

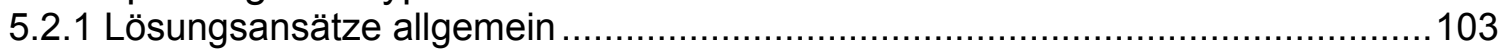

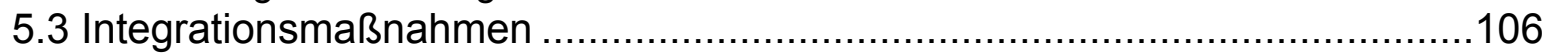

5.3.1 Fallstudie Göttingen............................................................................. 107

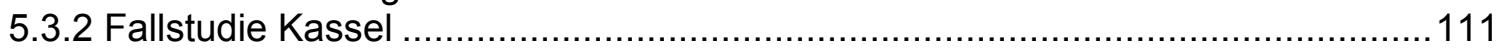

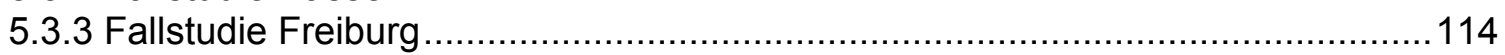

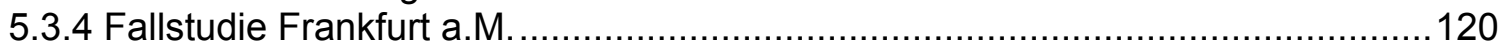

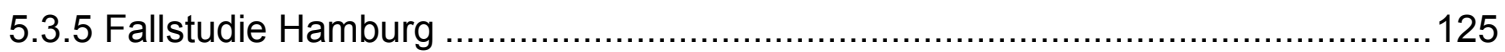

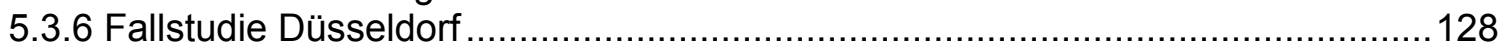

5.3.7 Fallstudie Berlin ................................................................................. 133

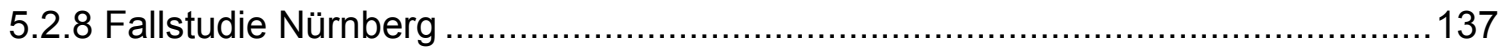

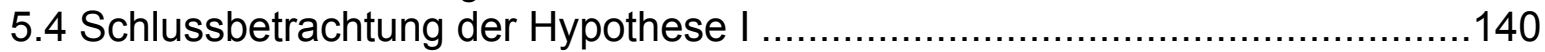

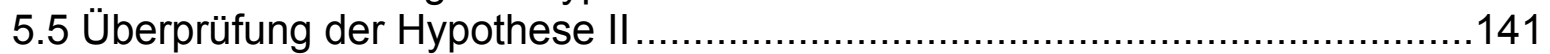

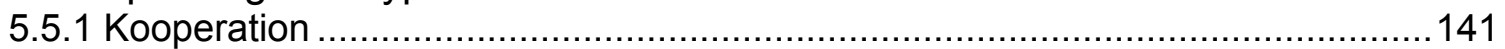

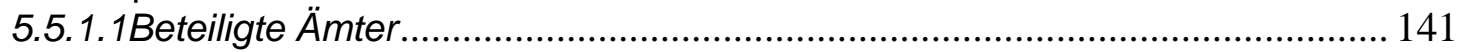

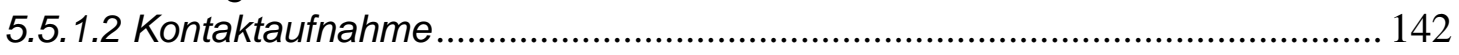

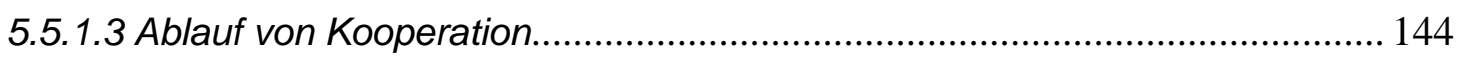

5.5.1.4 Entstehung von Kooperation ...................................................................... 147

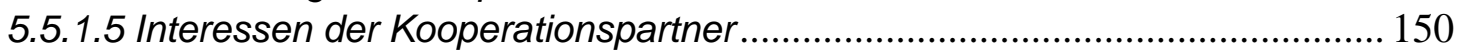

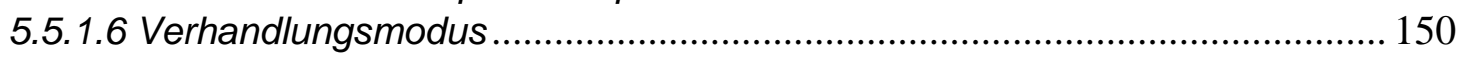

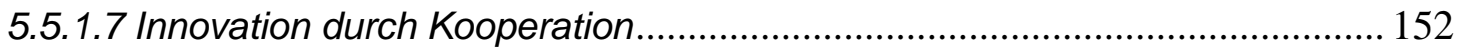

5.5.1.8 Merkmale einer erfolgreichen, integrativen Maßnahme................................. 153

5.5.1.9 Nutzen von Kooperation .............................................................................. 157

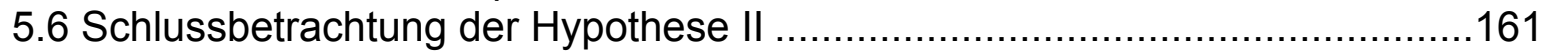

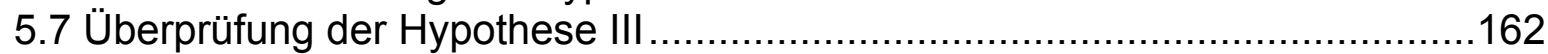

5.7.1 Neues Handlungsfeld / Produkt..................................................................... 162

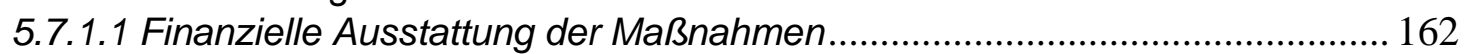

5.7.1.2 Personelle Ausstattung der Maßnahmen ....................................................... 165

5.7.1.3 Erschließung eines neuen Handlungsfeldes .................................................. 166

5.7.1.4 Aufgabenerweiterung............................................................................... 167

5.8 Schlussbetrachtung der Hypothese III ................................................168

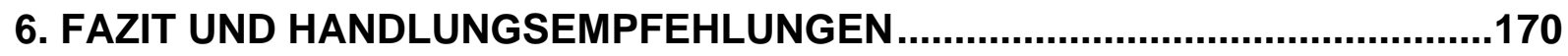

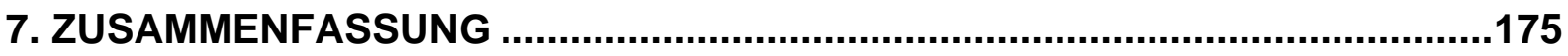

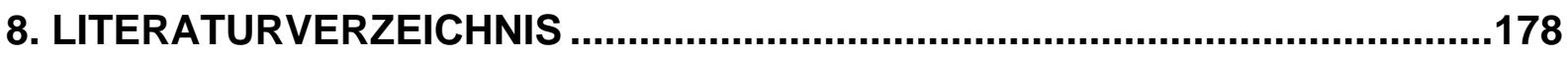

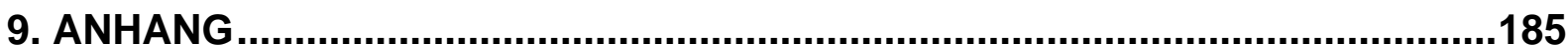




\section{Abbildungsverzeichnis}

Abbildung 1: Ebenen der Demokratie ( vgl. Fuchs, 1999, S. 165) ................................... 41

Abbildung 2: Liberales Modell der sozialen Integration (nach Fuchs, 1999, S. 169) ...........42

Abbildung 3: Prozessebene des Liberalen Modells der sozialen Integration .......................42

Abbildung 4: Forschungsansatz: Forstverwaltungshandeln für Liberale Integration............61

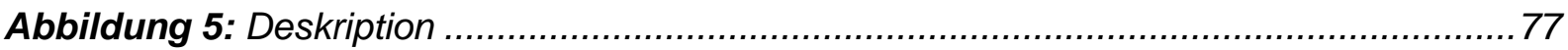

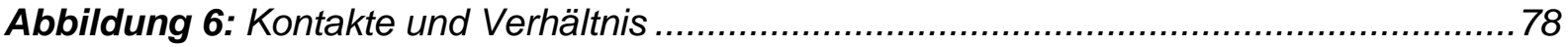

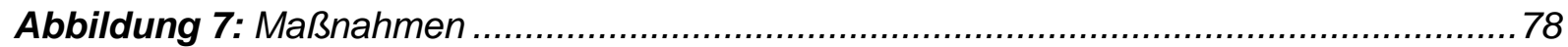

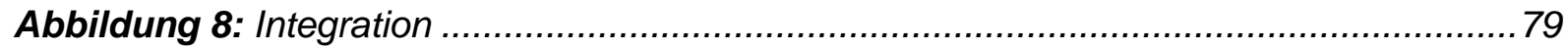

Abbildung 9: Mehrdimensionalität von Kooperation ................................................. 80

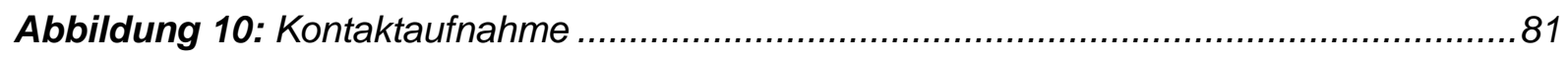

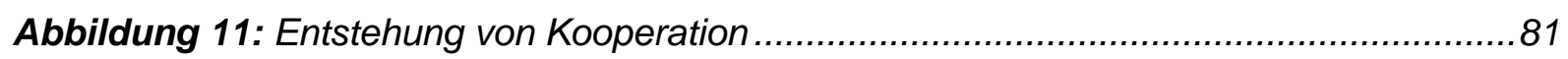

Abbildung 12: Interessen von Akteuren .............................................................. 82

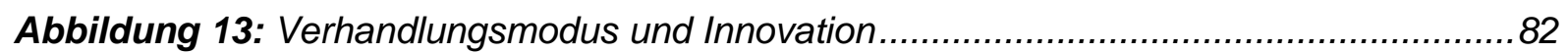

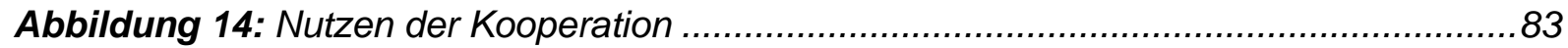

Abbildung 15: Ausstattung der Maßnahmen und Aufgabenerweiterung .............................8 84

Abbildung 16: Allgemeines inhaltsanalytisches Ablaufmodell (Mayring, 1997, S. 54) .........90

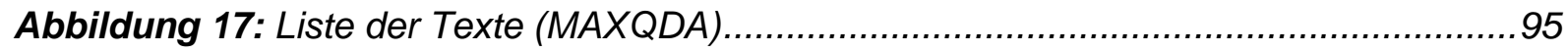

Abbildung 18: Beispiel für die Kategorisierung der Texte (MAXQDA) ................................96

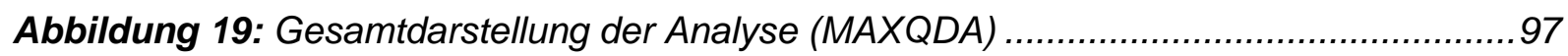




\section{Tabellenverzeichnis}

Tabelle 1: Vorteile und Nutzen von urbanem Wald und Bäumen (Tyrväinen et al., 2005, S. 82 übersetzt durch Verf.).

Tabelle 2: Indikatoren des sechsten Kriteriums und Erläuterungen (MCPFE, 2002, Übersetzung durch Verf.).

Tabelle 3: Erste Ebene der sozialen Nachhaltigkeit und deren Leitindikatoren 19

Tabelle 4: Zweite Ebene der sozialen Nachhaltigkeit und deren Leitindikatoren 19

Tabelle 5: Dritte Ebene der sozialen Nachhaltigkeit und deren Leitindikatoren 20

Tabelle 6: Vierte Ebene der sozialen Nachhaltigkeit und deren Leitindikatoren 20

Tabelle 7: Kommunale Ämter der sozialen Dienste und deren Hauptaufgaben. 22

Tabelle 8: Typen der Sozialintegration von Migranten (ebd., S. 19) 23

Tabelle 9: Ausgewählte Anlässe, Zielgruppen und Handlungstypen sozialer Hilfs- und Unterstützungsleistungen.

Tabelle 10: Wichtigste Merkmale der drei Modelle der Drogenpolitik (vgl. Jungblut, 2005, S. 285) 28

Tabelle 11: Ausgewählte Expertlnnen nach Fachbereichen 69

Tabelle 12: Strukturdaten der Städte (Quellen: Websites der aufgeführten Städte) .75

Tabelle 13: Institutionen in Göttingen, deren Maßnahmen und Partner 107

Tabelle 14: Institutionen in Kassel, deren Maßnahmen und Partner. 111

Tabelle 15: Institutionen in Freiburg i.Br., deren Maßnahmen und Partner....... 114

Tabelle 16: Institutionen in Frankfurt a. M., deren Maßnahmen und Partner. 120

Tabelle 17: Institutionen in Hamburg, deren Maßnahmen und Partner. 125

Tabelle 18: Institutionen in Düsseldorf, deren Maßnahmen und Partner. 128

Tabelle 19: Institutionen in Berlin, deren Maßnahmen und Partner 133

Tabelle 20: Institutionen in Nürnberg, deren Maßnahmen und Partner 137

Tabelle 21: Erfolg der Maßnahmen 153

Tabelle 22: Erfolgsfaktoren der Maßnahmen 154

Tabelle 23: Finanzierungsformen der Projekte und Anzahl der Nennungen. 163 


\section{Einleitung}

In der Bundesrepublik Deutschland ist das Thema Integration seit Jahren ein zentraler Diskussionspunkt der Politik. Die Bundesregierung hat sich 2006 für einen Nationalen Integrationsplan ausgesprochen. Im Vordergrund des Plans steht eine nachhaltige Integrationspolitik, die mit allen Ebenen, Bund, Ländern und Kommunen und den Akteuren der Bürgergesellschaft und Migrantinnen und Migranten erarbeitet werden soll. Es sollen klare Ziele, konkrete Maßnahmen und Selbstverpflichtungen der staatlichen und nichtstaatlichen Akteure in diesem Integrationsplan verankert werden. Der zweite Integrationsgipfel wird am 12. Juli diesen Jahres stattfinden und den Nationalen Integrationsplan vorstellen. Zuvor haben sich die Akteure in Arbeitsgruppen mit verschiedenen Themen zur Integration auseinandergesetzt. In diesen Arbeitsgruppen sind Empfehlungen erarbeitet worden, die in den Plan einfließen werden. Die Empfehlungen beziehen sich u.a. auf eine gezielte und verbesserte Sprachförderung, auf eine gute Bildung und Ausbildung, auf die positive Wirkung von kultureller Pluralität und der Integration durch Bürgerschaftliches Engagement (Bundesregierung, 2006). Eine Ausrichtung in den Integrationsbemühungen auf Migratinnen und Migranten ist in der Öffentlichkeit deutlich zu erkennen. Die Diskussion um die neue Unterschicht, das „Prekariat“, hat auch die Notwendigkeit von Integrationsmaßnahmen für andere Gesellschaftsgruppen hervorgehoben. Der 2. Armuts- und Reichtumsbericht der Bundesregierung legt offen, dass Armut und soziale Ausgrenzung nicht mehr als Randphänomen zu bewerten sind und auf einen bestimmten Bevölkerungsteil zutreffen, sondern alle Gesellschaftsschichten betreffen (Bundesministerium für Gesundheit und Soziale Sicherung, 2005a). Die politische Reaktion auf die soziale Lage in Deutschland basiert auf einer Verbesserung von Teilhabe- und Verwirklichungschancen der Bevölkerung. Um am gesellschaftlichen Leben in sozialer und ökonomischer Hinsicht teilzunehmen, müssen Armut und soziale Ausgrenzung gemindert werden. Die Armut in Deutschland korrespondiert deutlich mit der Arbeitslosigkeit. Die Betroffenen sind abhängig von finanziellen Hilfesystemen und sollen durch gezielte Maßnahmen wieder in den Arbeitsmarkt integriert werden. Mit der Reform der Grundsicherung für Arbeitssuchende (SGB II) werden Anreize und Chancen geschaffen, die den Weg aus der Langzeitarbeitslosigkeit weisen sollen. Hierzu zählen vor allem die

intensive Beratung, Vermittlung und individuellen Eingliederungshilfen und die Aktivierung der Betroffenen durch das Leistungssystem (Zuverdienstmöglichkeiten oder auch Kürzungen bei Ablehnung von Arbeitsangeboten) (ebd.). Von Armut und sozialer Ausgrenzung sind neben Arbeitslosen auch die Familien betroffen. Auch hierfür stehen Hilfsmaßnahmen zur Verfügung, die eine Verbesserung der Situation herbeiführen können. Zu nennen ist hier vor 
allem die finanzielle Entlastung der Familien. Durch die Erhöhung des Kindergeldes, die steuerliche Entlastung durch die Geltendmachung von erwerbsbedingten Betreuungskosten, einen Kinderzuschlag von 140 EURO für ALG II Empfänger und den Ausbau der Tagesbetreuung für Kinder sollen Familien vor den Risiken der Armut und Ausgrenzung (z.B. vom Berufsleben) bewahrt werden. Vor Ausgrenzung und Armut soll die Investition in Bildung schützen. Denn nur mit angemessenen und guten Berufs- und Bildungsabschlüssen ist ein Weg zur Positionierung auf dem Arbeitsmarkt und finanzieller Unabhängigkeit geebnet. Neben den Bemühungen der Bundesregierung auf dem Arbeitsmarkt (z.B. Ausbildungspakt zwischen Bundesregierung und Wirtschaft) trägt eine Wohnraumförderung für einkommensschwache Haushalte zur Verminderung von Armut und sozialer Ausgrenzung bei. Hierunter fallen die Wohngeldzahlungen und Projekte wie „Die Soziale Stadt", die eine Verbesserung der Lebenssituation Betroffener in benachteiligten Stadtteilen zum Ziel haben. Die Maßnahmen zur Verbesserung der Lebenssituation von Menschen mit Behinderung zielen auf die Integration in den Arbeitsmarkt und auf ein selbstständiges und selbstbestimmtes Leben ab. Besonders Suchtkranke, Obdachlose und Straffällige sind von extremer Armut und sozialer Ausgrenzung betroffen. Diesen Personengruppen kann durch die Bereitstellung von Wohnungen, den Zugang zur medizinischen Versorgung, Beratungsund Betreuungsangebote und finanzielle Unterstützung geholfen werden, die individuelle Situation zu überwinden und vor extremer Armut und Ausgrenzung schützen (ebd.). Diese Darstellung der Lebenslagen in Deutschland und die Maßnahmen, die von der Bundesregierung zur Verbesserung der Lebenssituationen ergriffen werden, weisen eindrücklich darauf hin, dass es sich bei der Integration nicht nur um die Eingliederung von Menschen mit Migrationshintergrund handelt. Der immense Bedarf an Maßnahmen wird angesichts der sich wandelnden Lebensumstände der Bevölkerung deutlich.

Da es sich bei der Integration von Problemgruppen um eine gesellschaftliche Aufgabe handelt, die sich über alle staatlichen Ebenen erstreckt, sehen sich die großen Kommunen mit dieser Aufgabe konfrontiert. Die Kommunen stehen dem Problem der ständig steigenden Sozialleistungen gegenüber, die bei einem bundesweiten durchschnittlichen Haushaltsdefizit von 5,2 Mrd. EURO kaum zu finanzieren sind (Stadt Bonn, 2006). Da ein gesamtgesellschaftlicher Nutzen durch die Integration entsteht, müssen Akteure in die Integrationsmaßnahmen einbezogen werden, die diese nicht originär in der Aufgabenwahrnehmung verankert sehen, z.B. die Forstverwaltungen. Gerade die Forstverwaltungen in urbanen Zentren haben mit einer Entwicklung zu kämpfen, die in den letzten Jahren eine abnehmende Tendenz der Bedeutung und des Einflussbereiches der Forstverwaltungen erkennen lässt. Durch die immensen Haushaltsdefizite der Stadtverwaltungen ist ein Bürokratieumbau und -abbau in den Großstädten zunehmend an der Tagesordnung (Pelz, 1996). Von diesem Um- und Abbau von Verwaltungseinheiten 
bleibt die Forstverwaltung nicht verschont. Vor dem Hintergrund dieser Ausführungen lässt sich die Fragestellung für die hier vorliegende Arbeit ableiten: Können die Forstverwaltungen in urbanen Zentren einen Beitrag zur sozialen Integration von Problem- bzw. Randgruppen leisten?

Zur Klärung der Frage ist es notwendig, einleitend die Bedeutung von urbanen Grün- und Waldflächen, die Diskussion um die soziale Nachhaltigkeit und die Akteure und Aspekte der Integrationsarbeit zu beleuchten (Kap. 1). Hieran schließt sich die Darstellung des Forschungsansatzes und der theoretischen Grundlagen der vorliegenden Arbeit an (Kap. 2 und 3). Aus den theoretischen Grundlagen werden die Hypothesen, die es zu überprüfen gilt, abgeleitet. Im Kapitel 4 sind die empirische Datenerhebung und deren Auswertungsverfahren beschrieben. Im anschließenden Ergebnisteil (Kap. 5) werden die empirischen Befunde nach Fallstudien betrachtet, theoriegeleitet interpretiert und diskutiert. Das abschließende Kapitel 6 soll die Folgen der Untersuchung für die kommunalen Forstverwaltungen offen legen und Handlungsempfehlungen liefern.

\subsection{Bedeutung von urbanen Grün- und Waldflächen}

Die Interessen am Wald sind ganz unterschiedlicher Natur. Waldeigentümer haben das Interesse des Bestandes der eigenen Herrschaft, also die Einflussnahme von außen so gering wie möglich zu halten und eigene Entscheidungen durchzusetzen. Der Erhaltenswert zählt ebenfalls zu den Interessen des Waldeigentümers, denn er ist darauf gedacht, sein Waldeigentum auf Dauer zu erhalten. Als weiteres Interesse des Eigentümers ist der Ertragswert des Waldes zu nennen, denn jeder Waldbesitzer ist darauf bedacht, wirtschaftliche Erträge zu erzielen. Die wirtschaftliche Lage eines Waldeigentümers kann sich auch durch den Verkauf des Waldes verändern (Zuwachs des Vermögens), so dass der Verkaufwert des Waldes ein weiteres Interesse darstellt. Der Liebhaberwert als Interesse der Eigentümers bezieht alle Interessen ein, die nicht direkt wirtschaftlich umrechenbar sind, wie z.B. der Prestigewert des Waldes (Krott, 2001, S. 36ff.). Neben den Interessen der Waldeigentümer stellen die Bürger eine weitere Interessensgruppe dar. Als Bürger werden „alle Mitglieder einer politischen Gemeinschaft“ (Krott, 2001, S. 48) bezeichnet. Sie kennzeichnet der direkte Zugriff auf den Wald und die Gestaltung der wirtschaftlichen und politischen Aktivitäten. Das Interesse des Bürgers am Wald bezieht sich vor allem auf den Freizeitbereich und nicht auf die Holzproduktion und Nutzung, da hierzu den meisten Bürgern der Bezug fehlt. Eine Vielfalt und die Konflikthaftigkeit sind die prägnantesten Merkmale der Bürgerinteressen. Je nach Milieu (Lebenswelt), dem die Bürger angehören, unterscheiden sich die Nutzungsinteressen deutlich voneinander. So nutzen Angehörige des 
„Niveaumilieus“ (über 50 Jahre, hohes Bildungsniveau, Handlungsstil von Ordnung geprägt) den Wald als Augleich für den oftmals stressigen Alltag und besuchen ihn häufig an den Wochenenden, wobei dieser von Menschen möglichst unbeeinflusst sein sollte (Braun, 2000, S. 183). Für das „Integrationsmilieus“ (über 50 Jahre, kein hohes Bildungsniveau, Handlungsstil von Ordnung geprägt) ist der Waldbesuch selbstverständlich und gehört zur Lebenswelt der Personen. Die Waldbehandlung ist von ordnenden Strukturen und der Kontrolle durch den Menschen geprägt (ebd., S. 191). Das „Harmoniemilieu“ (über 50 Jahre, niedriges Bildungsniveau, Handlungsstil weit mehr von Ordnung geprägt als das des "Integrationsmilieus“) empfindet die Gesellschaft als negativ und den Wald als Ausgleich dazu besonders positiv. Die Waldbesuche dieses Milieus sind sehr selten und meist von positiven Erlebnisse aus der Vergangenheit geprägt, welches mit einem Gefühl von Heimat verbunden wird (ebd., S. 198). Im „Selbstverwirklichungsmilieu“ (unter 50 Jahre, hohes Bildungsniveau, Handlungsstil von Spontaneität bzw. Selbstentfaltung geprägt) verliert der Wald an symbolischer Bedeutung und da er nur eingeschränkte Freizeitaktivitäten bietet, werden die Waldbesuche weniger bzw. die Distanz zum Wald größer. Dieses Milieu ist über die sachlichen Zusammenhänge des Waldes sehr gut informiert (ebd., S. 206). Die Wahrnehmung des Waldes im „Unterhaltungsniveau“ (unter 50 Jahre, niedrigeres Bildungsniveau als das "Selbstverwirklichungsmilieu“, Handlungsstil ist auch von Spontaneität geprägt) ist durch die Bedürfnisbefriedigung gekennzeichnet. Da der Wald die Bedürfnisse aber nicht befriedigen kann, ist die Distanz zum Wald sehr groß und das Leben auf die Stadt konzentriert (ebd., S. 214). Die Ausführungen zeigen, dass die gesellschaftlichen Gruppen ganz unterschiedliche Wahrnehmungen von Wald und Interessen am Wald haben. Alle Interessen von Waldnutzern sollten miteinander vereinbar und aufeinander abgestimmt sein. Die Aufgabe der Koordinierung der Interessen und Vorstellungen ist daher in Stadtwäldern bzw. stadtnahen Wälder besonders wichtig und schwierig zu lösen. Gerade in Wälder, die direkt an das Stadtgebiet angrenzen oder auch z.T. in der Stadt liegen, ist ein enormer Nutzungsdruck durch die Bevölkerung vorhanden. Der Interessensvielfalt kann die Forstwirtschaft, wie sie in ruralen Gebieten betrieben wird, wo die Holznutzung und die Produktion im Vordergrund stehen, nicht gerecht werden. Für die Bewirtschaftung des stadtnahen Waldes, wo eindeutig dessen Erholungs- und Schutzfunktion erhalten und gefördert werden soll, hat sich das „Urban Forestry“ - Konzept durchgesetzt. Dieses Konzept hat seinen Ursprung in den 60iger und 70iger Jahren in Nordamerika und unterliegt verschiedenen Definitionsvarianten. Die Stärken des Konzeptes „Urban Forestry“ lassen sich für Europa wie folgt zusammenfassen:

„It ist integrative, incorporating different elements of urban green structures into a whole (the 'urban forest')

It is strategic, aimed at developing longer-term policies and plans for urban tree resources, connecting to different sectors, agendas and programs.

It is aimed at dilivering multiple benefits, stressing the economic, environmental and socio-cultural goods and services urban forests can provide. 
It is multidisciplinary and aiming to become interdisciplinary, involving experts from natural as well as social science.

It is participatory, targeted at developing partnerships between stakeholders." (Randrup et al., 2005, S. 15)

Das Konzept ist auf die Planung, Entwicklung und die Bewirtschaftung von Wald in der Stadt und in stadtnaher Umgebung ausgelegt. Dies soll unter Berücksichtigung aller Disziplinen geschehen, die eine Verbindung zum Wald und urbanem Grün haben. Hierbei soll der größtmögliche Nutzen für alle Interessensvertretungen erreicht werden. Der Nutzen des urbanen Waldes und der Stadtbäume variiert durch die unterschiedlichen Entwicklungen hinsichtlich der Umwelt und dem sozio-kulturellen Hintergrund in Europa sehr stark. Dennoch können generelle Nutzenkategorien aufgestellt werden:

Tabelle 1: Vorteile und Nutzen von urbanem Wald und Bäumen (Tyrväinen et al., 2005, S. 82 übersetzt durch Verf.)

\begin{tabular}{|l|l|}
\hline Soziale Vorteile & $\begin{array}{l}\text { Erholungsmöglichkeiten, Verbesserung der häuslichen und Arbeitsumgebung, } \\
\text { Auswirkungen auf die physische und geistige Gesundheit. Kulturelle und geschichtliche } \\
\text { Werte von Grünräumen. }\end{array}$ \\
\hline $\begin{array}{l}\text { Ästhetische und } \\
\text { architektonische } \\
\text { Vorteile }\end{array}$ & $\begin{array}{l}\text { Landschaftliche Variationen durch verschiedene Farben, Beschaffenheit, Formen und } \\
\text { Dichte von Pflanzen. Wachstum von Bäumen, saisonale Veränderungen und erfahrbare } \\
\text { Natur. Offene Fläche definieren, Rahmen und Abschirmen der Sicht, Gebäude } \\
\text { landschaftliche gestalten. }\end{array}$ \\
\hline $\begin{array}{l}\text { Klimatische und } \\
\text { physische Vorteile }\end{array}$ & $\begin{array}{l}\text { Kühlen, Windkontrolle, Wirkung auf das urbane Klima durch Temperatur und } \\
\text { Feuchtigkeitskontrolle. Reduktion der Luftverunreinigung, Lärmkontrolle, Strahlen- und } \\
\text { Reflektionsreduktion, Vorbeugung vor Flut und Erosionskontrolle }\end{array}$ \\
\hline Ökologische Vorteile & Biotope für Flora und Fauna in städtischer Umwelt \\
\hline Ökonomische Vorteile & $\begin{array}{l}\text { Wert von Produkten (Holz, Beeren, Pilze etc) zu Marktpreisen, gestiegene } \\
\text { Eigentumswerte, Tourismus }\end{array}$ \\
\hline
\end{tabular}

Die Varianten von Nutzen und Vorteilen des städtischen Waldes und Grüns spiegeln die oben bereits erwähnte Konflikthaftigkeit der Nutzung wider. Sollen nun alle Interessen und Vorteile verwirklicht werden, bedarf es einem starken Konzept, welches in der Lage ist, die Ansprüche und Interessen auf kleinem Areal zu erfüllen und umzusetzen. Diese Aufgabe soll das Konzept Urban Forestry übernehmen durch die Einbeziehung verschiedener Disziplinen, die Interessen und Nutzungen aufeinander abstimmen sollten. Für die vorliegende Arbeit sind vor allem die Sozialen Vorteile bzw. der soziale Nutzen von Stadtwald und -grün von Interesse. So lässt sich das Spazierengehen im Wald als allgemein häufigste Erholungsmöglichkeit ausmachen (Tyrväinen et al., 2005, S. 84). Andere Freizeitaktivität, wie Fahrradfahren, Joggen, Picknicken, Beeren oder Pilze pflücken variieren in der Häufigkeit oder Intensität von Land zu Land in Europa, so ist das Fahrradfahren im Wald nicht sehr populär in Italien (ebd.). Das Vorhandensein von Wald und städtischem Grün führt weiterhin zu einem gesundheitlichen Vorteil für die Bevölkerung. Durch die Spende von Schatten und 
die Funktion als Luftfilter von Abgasen erhöhen Bäume und Wald die Qualität des Wohnumfeldes (ebd. S. 85f.). Ist die Wohnumgebung in direkter Nähe zu Wald oder Grünflächen halten sich die Anwohner häufiger draußen auf und sind eher geneigt sich sportlich zu betätigen, was sich positiv auf die Gesundheit auswirkt (ebd., S. 86). Ein positiver Effekt auf die Gesundheit durch den Wald ist in den sozialen Beziehungen zu sehen. Studien in den USA haben gezeigt, dass die soziale Beziehungen und Zusammenhalt von Menschen, die sich im Freien, in Grünräumen mit Bäumen, treffen positiv beeinflußt werden (ebd. S, 87). Das soziale Potential des Waldes soll vor allem den Ansprüchen der unterschiedlichen Nutzergruppen gerecht werden, die sich in den letzten Jahren stark verändert haben, z.B. aufgrund des demographischen Wandels leben mehr ältere Menschen in den Städten deren Ansprüche beachtet werden sollten (ebd.). Den wachsenden Bedarf an sozial integrativem Potential des Waldes stellten Germann-Chiari und Seeland (2004) fest, wobei die Integrationsmaßnahmen abhängig sein sollen vom sozialen Status der Menschen, dem Alter, dem Geschlecht und dem ethnischen Hintergrund bzw. auf die daraus erwachsenden Ansprüche abgestimmt sein sollen.

Krott (1998, S.9) zeigt auf, dass sich das Konzept der Urbanen Forstwirtschaft stark von dem der ruralen Forstwirtschaft unterscheidet. Die unterschiedlichen Ansprüche können im urbanen Raum nur mit innovativen Konzepten befriedigt werden. Die Problemlösung durch urbane Forstwirtschaft bedarf spezieller Programme für die Mehrfachnutzung und die Nutzer, geeignete Institutionen und spezielle Strategien für die Zusammenarbeit mit Interessengruppen. Für die Zusammenarbeit mit anderen Institutionen sollte die Forstverwaltung in den urbanen Zentren offen sein. Sie kann einen entscheidenden Beitrag leisten, ist aber nicht in der Lage, dies in Eigenleistung bzw. in Eigenverantwortung zu tun. Die Maßnahmen im Wald zur Integration von z.B. Suchtkranken oder älteren Menschen können in Zusammenarbeit mit den entsprechenden Ämtern ausgearbeitet und umgesetzt werden.

Ottitsch und Krott (2005) sprechen von einer „aufgabenorientierten unfassenden urbanen Forstwirtschaft", die allen Ansprüchen und Interessen gerecht werden sollte. Um dies zu erreichen werden Faktoren genannt, die neue Optionen für die Forstwirtschaft in urbanen Räumen aufzeigen können. Zum einen können dies marktorientierte Projekte sein, wie z.B. neue urbane „Vergnügungscenter“, neue Baugebiete am Stadtrand, Erneuerung von Stadtteilen innerhalb der Stadt. Die Herausforderung für die Forstwirtschaft besteht hierbei darin, dass erkannt wird, wie die Gestaltung von Bäumen und Wäldern diese Projekte positiv unterstützen kann und deren Ansprüchen gerecht werden kann. Die großen Vorteile einer Beteiligung der Forstwirtschaft bei Neubau- bzw. Umbauprojekten sind die immense finanzielle Grundlage und die politische Bedeutung der Projekte. Hinzu kommt die professionelle Planung, die innovative Konzepte unter Beteiligung vieler Institutionen Realität 
werden lässt (Ottitsch, Krott, 2005, S. 142f.). Ein weiterer Faktor, den Einfluss von urbaner Forstwirtschaft zu stärken, ist den symbolischen Charakter des Waldes zu nutzen. Das Ziel der Politik in einer Stadt ist u. a. den Zusammenhalt, das Zusammenleben und die Identität der Bevölkerung in und mit der eigenen Stadt zu fördern und aufrechtzuerhalten. Hierzu können die positiv besetzten Assoziationen von Wald beitragen. So kann der Wald zur Identität einer Stadt beitragen, auf den die Bewohner stolz sind und so die individuelle Bindung an die Stadt verstärkt wird. Durch die gemeinsame Sichtweise der Stadtbevölkerung wird der Zusammenhalt intensiviert (ebd., S. 144f.). Die Integration von Randgruppen kann ebenfalls einen Faktor darstellen, der zu einer neuen Aufgabe der urbanen Forstwirtschaft werden kann. Gerade in Städten nehmen auch sog. Randgruppen bzw. Minderheiten den Wald in Anspruch. Die Aufgabe der Forstverwaltung könnte darin bestehen, die Sozialpolitik der Stadt so mitzugestalten, dass die Ansprüche von Minderheiten an den Wald erfüllt werden können und somit eine Integration in die Gesellschaft stattfinden kann, z.B. durch Bereitstellen von Grillplätzen und Treffpunkten für Ausländer. Zudem kann die Forstverwaltung zu einem Bündnispartner oder Interessensvertreter von Randgruppen werden und sich für deren Rechte und Ansprüche stark machen. Hierdurch erlangen sie politischen Einfluss und sind in der Lage die eigenen Interessen zu artikulieren und zu vertreten. Werden die Problemgruppen von Institutionen unterstützt, können sie sich an politischen Entscheidungen innerhalb der Stadt, die sie betreffen, beteiligen. Der Partizipierungsprozess kann nicht allein von der Forstverwaltung getragen und forciert werden, sondern bedarf der Zusammenarbeit von entsprechenden öffentlichen Institutionen (ebd., S. 145). Auch Konijnendijk (2000) stellt heraus, dass die Forstwirtschaft in Städten durch die sich wandelnden Aufgaben von Partnern und anderen Berufsgruppen lernen und sich anderen Disziplinen öffnen sollten. Dies ist eine Voraussetzung für die Schaffung neuer Produkte und Dienstleistungen, die auf die Ansprüche der Stadtbevölkerung zugeschnitten sein sollen. Die besondere Stellung und Aufgabenerfüllung der Forstverwaltung innerhalb einer Stadt soll das Beispiel des Städtischen Forstamtes Freiburg i.Br. aufzeigen. Das Forstamt Freiburg ist als eigenständige Verwaltungseinheit in der gesamten Stadtveraltung zu bezeichnen. Dies stellt bis heute eine Ausnahme dar, da die meisten Großstädte in Deutschland keine eigenen Forstverwaltungen unterhalten und diese an z.B. die Umweltoder Grünflächenämter der Stadt angegliedert haben. Burgbacher (1996) spricht von der Interessensvielfalt und -inhomogenität bezüglich des Waldes. Um diese Interessen möglichst ausreichend zu erfüllen, bedarf es ständigen Abstimmungsprozessen unter den Verantwortlichen mit der Verwaltungsspitze, dem Gemeinderat und der übrigen Bürgerschaft. Das Forstamt in Freiburg ist neben dem Umweltschutzamt, dem Gartenamt und dem Eigenbetrieb Abfallwirtschaft dem Umweltdezernat zugeordnet, dessen Leitung der Umweltbürgermeister innehat. Die wichtigen Fragen der einzelnen Fachverwaltungen 
werden in wöchentlichen Besprechungen aufeinander abgestimmt und hierbei informiert der Bürgermeister die Amtsleiter über die aktuellen stadtpolitischen Entscheidungen. Sind Entscheidungen innerhalb der Forstverwaltung zu fällen, so werden sie zunächst mit dem Dezernat und den beteiligten Ämtern besprochen und dem zuständigen Fachgremium vorgetragen. Neben den Gemeinderäten werden hierzu auch die Naturschutzverbände und Naturschutzbeauftragten hinzugezogen. Dieses Prozedere versetzt die Forstverwaltung in die Lage, fundierte Argumente für die Entscheidungen vorzubringen und so Akzeptanz und Vertrauen auf allen politischen Ebenen zu erzielen. Die städtischen Forstverwaltungen stehen in der Regel im Zwiespalt die Holzproduktion voranzutreiben, weil damit eine gesamtstädtische Einnahmequelle verbunden ist und die Schutz- und Erholungsfunktion des Waldes zu erhalten und zu verbessern. Die sehr kostenintensiven Maßnahmen zur Erhaltung und Verbesserung z.B. von Erholungseinrichtungen im Wald, kann z.T. nur aus den Einnahmen der Holznutzung finanziert werden. Hierzu bedarf es einer Zielrangfolge der Forstverwaltungen, die in Freiburg wie folgt umgesetzt wurde:

- „Optimierung der Waldfunktionen durch standorts- und funktionenbezogene Schwerpunktsetzung;

- Beschränkung der Holznutzung auf Flächen, auf denen sie aufgrund der standörtlichen Bedingungen ökonomisch sinnvoll ist.

- Ausrichtung von Holzproduktion und -nutzung in Art und Umfang an ökologischen Gesichtspunkten (nicht nur als Konzession an den Naturschutz, sondern vor allem als ökonomisches Gebot zur Vermeidung der Folgekosten einer naturfernen, risikobehafteten und damit unrentablen Waldbewirtschaftung);

- Ausscheidung von Vorrangflächen für Naturschutzziele mit Verzicht auf Holznutzung;

- gezielte Gestaltungsmaßnahmen an Erholungsschwerpunkten unter Nachrangigkeit von Naturschutz- und Nutzungszielen." (Burgbacher, 1996, S. 1096)

Diese Zielrangfolge für den Stadtwald Freiburg macht deutlich, wie die unterschiedlichen Funktionen des Waldes aufeinander abgestimmt und gleichwertig nebeneinander existieren und umgesetzt werden können.

Die vorhergegangen Ausführungen sollen die Besonderheiten in der Bewirtschaftung des Stadtwaldes herausstellen. Besonders zu beachten ist hierbei die stets erwähnte Vielfalt der Interessen, denen der Wald und damit die Forstverwaltung, als eigenständige Verwaltung oder als Fachbereich innerhalb einer Stadt gerecht werden muss. Deutlich sollte auch werden, dass die Forstverwaltungen den Einfluss auf die städtische Entwicklung nur mit an die Nachfrage angepassten Angeboten und Dienstleistungen erhalten können. Aufgrund der sich verändernden Ansprühe an den Wald und die sich ebenfalls verändernde Stadtbevölkerung muss die Forstveraltung die Sozialleistung des Waldes neu überdenken. 


\title{
1.2 Soziale Nachhaltigkeit
}

Die Forstwirtschaft prägt die Diskussion um die Nachhaltigkeit seit Beginn des 18. Jahrhunderts (Schanz, 1996, S. 20). Ein Zitat von Georg-Ludwig Hartig aus dem Jahre 1795 soll verdeutlichen, welche Dimensionen die Nachhaltigkeit aus früherer Sicht besaß:

\begin{abstract}
„Es läßt sich keine dauerhafte Forstwirtschaft denken und erwarten, wenn die Holzabgabe aus den Wäldern nicht auf Nachhaltigkeit berechnet ist. Jede weise Forstdirektion muß daher die Waldungen die Staates ohne Zeitverlust taxieren lassen und sie zwar so hoch als möglich, doch so zu benutzen suchen, daß die Nachkommenschaft wenigstens ebenso viel Vorteil daraus ziehen kann, als sich die jetzt lebende Generation zueignet." (Georg-Ludwig-Hartig Stiftung, 2007)
\end{abstract}

Der Schwerpunkt liegt hier auf der ökonomischen Dimension von Nachhaltigkeit, wobei die ökologische Dimension auch berücksichtigt wird. Nachhaltigkeit wird zudem von weiteren Aspekten geprägt (Schanz, 1996, S. 22), sozialen, zeitlichen und räumlichen Aspekten. Ist demnach von Nachhaltigkeit der Forstwirtschaft die Rede, werden meist alle fünf o.g. Dimensionen angeschnitten (ebd., S.23). Einen europäischen Standard für die nachhaltige Bewirtschaftung des Waldes liefert eine einheitliche Definition der Ministerkonferenz zum Schutz der Wälder in Europa (MCPFE):

„[...] „sustainable management“ means the stewardship and use of forests and forest land in a way, that maintains their biodiversity, productivity, regeneration capacity, vitality and their potential to fulfil, now and in the future, relevant ecological, economic and social functions, at local, national and global levels, and that does not cause damage to other ecosystems." (MCPFE, 1993)

Um die Nachhaltige Waldbewirtschaftung europaweit umsetzen zu können, wurden sechs Kriterien erarbeitet, an denen die Nachhaltige Bewirtschaftung von Wäldern gemessen wird und die als wichtiges Instrument der europäischen Forstpolitik gelten. Nachfolgend sind die Kriterien einer Nachhaltigen Waldbewirtschaftung aufgelistet:
1. Erhaltung und angemessene Verbesserung der forstlichen Ressourcen und ihr Beitrag zu globalen Kohlenstoffkreisläufen.
2. Erhaltung der Gesundheit und Vitalität von Forstökosystemen.
3. Erhaltung und Förderung der Produktionsfunktionen der Wälder (Holz- und Nichtholzprodukte).
4. $\quad$ Erhaltung, Schutz und angemessene Verbesserung der Biodiversität in Forstökosystemen.
5. $\quad$ Erhaltung und angemessene Verbesserung der Schutzfunktion bei der Waldbewirtschaftung (v.a. Boden und Wasser).
6. $\quad$ Erhaltung anderer sozio-ökonomischer Funktionen und Bedingungen.

(Deutscher Forstwirtschaftsrat, 2006)

Anhand der Kriterien wird deutlich, dass die drei Hauptdimensionen der Nachhaltigkeit, nämlich die ökologische, ökonomische und soziale, Berücksichtigung finden. Die einzelnen Kriterien werden wiederum durch Indikatoren spezifiziert. So wird das erste Kriterium z.B. durch die Waldfläche und den Zuwachs quantifiziert. Der Aspekt der sozialen Nachhaltigkeit wird im sechsten Kriterium aufgegriffen und durch folgende Indikatoren beschrieben: 
Tabelle 2: Indikatoren des sechsten Kriteriums und Erläuterungen (MCPFE, 2002, Übersetzung durch Verf.)

\begin{tabular}{|c|c|c|}
\hline Nr. & Indikator & Erläuterungen \\
\hline 6.1 & Waldbesitz & Anzahl der Waldbesitzer, eingestuft nach Besitzart und Größe \\
\hline 6.2 & $\begin{array}{l}\text { Beitrag des } \\
\text { Forstsektors zum BIP }\end{array}$ & $\begin{array}{l}\text { Beitrag der Forstwirtschaft und der Produktion von Holz- und Papierprodukten } \\
\text { zum BIP (Bruttoinlandsprodukt) }\end{array}$ \\
\hline 6.3 & Nettoeinkommen & Nettoeinkommen der Forstbetriebe \\
\hline 6.4 & $\begin{array}{l}\text { Aufwendungen für } \\
\text { Serviceleistungen }\end{array}$ & $\begin{array}{l}\text { Gesamtaufwendungen für langfristige, nachhaltige Serviceleistungen aus } \\
\text { Wäldern }\end{array}$ \\
\hline 6.5 & $\begin{array}{l}\text { Arbeitskraft des } \\
\text { Forstsektors }\end{array}$ & $\begin{array}{l}\text { Zahl der Beschäftigten und Arbeitsaufwand im Forstsektor, eingestuft nach } \\
\text { Geschlecht und Altersgruppe, Ausbildungs- und Arbeitsplatzeigenschaften }\end{array}$ \\
\hline 6.6 & $\begin{array}{l}\text { Arbeitssicherheit und } \\
\text { Gesundheit }\end{array}$ & Häufigkeit der Arbeitsunfälle und Berufskrankheiten in der Forstwirtschaft \\
\hline 6.7 & Holzverbrauch & Verbrauch von Holz und Holzprodukten pro Kopf \\
\hline 6.8 & Holzhandel & Im- und Export von Holz und Holzprodukten \\
\hline 6.9 & $\begin{array}{l}\text { Energie aus } \\
\text { Holzressourcen }\end{array}$ & $\begin{array}{l}\text { Anteil der Energie aus Holz vom Gesamtenergieverbrauch, eingestuft nach } \\
\text { Herkunft des Holzes }\end{array}$ \\
\hline 6.10 & $\begin{array}{l}\text { Zugänglichkeit für } \\
\text { Erholung }\end{array}$ & $\begin{array}{l}\text { Walflächen und andere bewaldete Flächen, auf denen die Öffentlichkeit das } \\
\text { Recht auf Zutritt hat zum Zwecke der Erholung und Angabe der } \\
\text { Nutzungsintensität }\end{array}$ \\
\hline 6.11 & $\begin{array}{l}\text { Kulturelle und } \\
\text { spirituelle Werte }\end{array}$ & $\begin{array}{l}\text { Zahl der Plätze in Wäldern und bewaldeten Flächen, die einen ausgewiesenen } \\
\text { kulturellen und spirituellen Wert besitzen }\end{array}$ \\
\hline
\end{tabular}

Bei diesem Kriterium handelt es sich um die Erhaltung der sozio-ökonomischen Funktionen und Bedingungen des Waldes, wobei der Schwerpunkt auf den ökonomischen Funktionen liegt. Diese Indikatoren haben durch die ökonomische Ausrichtung immer auch Einfluss auf das soziale Leben, denn ohne Einkommen aus dem Wald können soziale Standards nicht aufrechterhalten werden.

Schanz (1994, S. 82) stellt in seiner Untersuchung fest, dass die Forstleute im Begriff „Forstliche Nachhaltigkeit“ wichtige Besonderheiten des Berufsstandes sehen, wie eine gesamtgesellschaftliche Verantwortung, eine Langfristigkeit im Denken und den Aspekt des Bewahrens. Es schließt sich hier die Frage an, ob die gesamtgesellschaftliche Verantwortung durch die Erhaltung der Schutz- und Erholungsfunktionen erfüllt ist. Diese Schutz- und Erholungsfunktionen des Waldes werden von Speidel (1984) als Infrastrukturleistungen und Teil der „dynamischen Nachhaltigkeit“" (ebd., S. 44) angesehen, wobei die dynamische Nachhaltigkeit die Fortdauer einer Leistung beschreibt. Dass die Forstwirtschaft für die Nachhaltigkeit auf multifunktionaler Ebene verantwortlich ist, macht die Definition von Nachhaltigkeit nach Speidel (1984, S. 43) deutlich:

„Als „Nachhaltigkeit“ soll die Fähigkeit des Forstbetriebes bezeichnet werden, dauernd und optimal Holznutzungen, Infrastrukturleistungen und sonstige Güter zum Nutzen der gegenwärtigen und künftigen Generationen hervorzubringen. Danach sind diejenigen Handlungen und Leistungen "nachhaltig“, die dem Forstbetrieb diese Fähigkeit verschaffen und/oder dauernd erhalten." 
Auch das Niedersächsische Ministerium für den ländlichen Raum, Ernährung, Landwirtschaft und Verbraucherschutz greift in der Nachhaltigkeitsdiskussion auf die Vielfachnutzung des Waldes zurück und setzt diese mit der Nachhaltigkeit der Schutz- und Erholungsfunktion gleich (Niedersächsisches Ministerium für den Ländlichen Raum, Ernährung, Landwirtschaft und Verbraucherschutz, 2007). Das „Nachhaltigkeitsdreieck“ gibt hier zunächst Auskunft über die Ziele der Nachhaltigkeit:

- „ökologische Nachhaltigkeit = die Bewahrung der Vielfalt der Schöpfung mit den natürlichen Lebensgrundlagen für jetzigen und künftige Generationen

- soziale Nachhaltigkeit = für Alle sollen die gleichen Chancen in Bezug auf Wohlstand, Bildung und Kultur gelten

- $\quad$ ökonomische Nachhaltigkeit = die leistungsfähige Wirtschaft, die keine Probleme für die nachfolgenden Generationen hinterlässt“ (Niedersächsisches Ministerium für den Ländlichen Raum, Ernährung, Landwirtschaft und Verbraucherschutz, 2007)

Interessant ist nun die Ableitung der Nachhaltigkeitsdimensionen aus forstlicher Sicht. Sie werden mit der Nachhaltigkeit der Holzerträge, der Holzerzeugung, der Gelderträge und eben der Vielfachnutzung gleichgesetzt (ebd.). Hier wird deutlich, dass die soziale Nachhaltigkeit nicht näher beschrieben wird, sondern mit der Schutz- und Erholungsfunktion des Waldes abgedeckt ist. Als ausgewiesene Waldfunktionen (Wullschleger, 1982, Leibundgut, 1975, S. 148ff.) ist die Schutzfunktion des Waldes greifbar und kann mit Indikatoren beschrieben und gemessen werden, z.B. Schutzfunktion gegen Erosion, Lärm, Abgase, Wasserverschmutzung etc. (Wullschleger, 1982 S. 9). Die Erholungsfunktion dagegen wird meist nur genannt und nicht mit Inhalt gefüllt (Leibundgut, 1975, S. 150), wobei im Allgemeinen darunter das Erholen im Wald in jeglicher Form, z.B. Spazierengehen, Sport etc., gemeint ist. Leibundgut (1975, S. 13) fasst den Erholungsraum Wald, seine Rolle als Landschaftselement und die Schutzwirkungen des Waldes als Sozialfunktionen des Waldes zusammen und weist darauf hin, dass diese Funktionen nachhaltig zu sichern sind. Dieterich (1953) greift in seiner Funktionenlehre ebenfalls die Wohlfahrtswirkungen des Waldes auf, die in der Bewirtschaftung Berücksichtigung finden sollen. Er zieht hier die Arbeitsfunktion des Waldes hinzu, die den Menschen wirtschaftliche und soziale Vorteile bringt (ebd., S. 18). Die Menschen können wichtige Bedürfnisse befriedigen, die im ländlichen Raum z.B. in der Brennholzbeschaffung und im urbanen Raum in der Erholung zu sehen sind (ebd., S.18 u. 22). Ist es aber legitim, die Schutz- und Erholungsfunktion des Waldes mit der sozialen Nachhaltigkeit und sozialen Waldfunktion gleichzusetzen? Zum einen ist es legitim, denn die Verbesserung der Wasser- und Klimaverhältnisse wirken sich gesamtgesellschaftlich aus, aber die ökologischen und ökonomischen Argumente der Forstseite reichen, nach Meinung der Autorin, nicht aus, um die soziale Nachhaltigkeit ausreichend zu definieren.

Im Zuge der unklaren Definition von sozialer Nachhaltigkeit erarbeiteten Empacher und Wehling (2002) einen Vorschlag für Indikatoren, welche die soziale Dimension von Nachhaltigkeit beschreiben und messbar machen. Unter Einbeziehung 
sozialwissenschaftlicher Theorie- und Wissensbestände, wie z.B. Grundbedürfnis Konzepte, sozialwissenschaftlicher Funktionalismus, Konzepte des Sozialkapitals und die Diskussion um Sozialindikatoren, Lebensqualität und Wohlfahrtswirkungen, sollten konkrete Indikatoren zur sozialen Nachhaltigkeit erarbeitet werden (ebd., S. 4). Für die unterschiedlichen Ebenen der sozialen Nachhaltigkeit werden je zwei Leitindikatoren vorgeschlagen, die als Stellvertreter für viele Phänomene bzw. Ausprägungen der Ebene stehen (z.B. in der Ökologie steht die CO2-Emmission als Leitindikator für den Ausstoß weiterer klimarelevanter Stoffe) (ebd., S. 65). Die Leitindikatoren sollen Auskunft über die objektiven und subjektiven Aspekte der Ebenen geben. Diese Ebenen der sozialen Nachhaltigkeit sollen im Folgenden mit den Leitindikatoren verdeutlicht werden:

Tabelle 3: Erste Ebene der sozialen Nachhaltigkeit und deren Leitindikatoren

\begin{tabular}{|c|c|c|}
\hline Ebene sozialer Nachhaltigkeit & & Indikator \\
\hline \multirow{2}{*}{$\begin{array}{l}\text { Befriedigung der Grundbedürfnisse / } \\
\text { Arbeit }\end{array}$} & objektiv & HPI 2 (Human Poverty Index 2) \\
\hline & subjektiv & Allgemeine Lebenszufriedenheit \\
\hline
\end{tabular}

Die objektive Beurteilung der Grundbedürfnisbefriedigung soll über den HPI 2 erfolgen, der Auskunft über die Armut in Industrieländern in Bezug zu den Bildungs-, Gesundheits-, Einkommens- und Beschäftigungssituationen gibt (ebd., S. 68) (der HPI 1 beschreibt diese Faktoren für die Entwicklungsländer). Beschrieben wird dieser Index durch den Prozentsatz der Menschen, deren Lese- und Schreibfähigkeit unzureichend ist, deren Lebenserwartung unter 60 Jahren liegt, deren Einkommen unter 50\% des Durchschnitteinkommens liegt und den Anteil der Langzeitarbeitslosen (ebd., S. 59). Um die Grundbedürfnisse zu befriedigen und somit zur sozialen Nachhaltigkeit beizutragen, sollte es Ziel sein, diesen HPI 2 Index möglichst gering zu halten. Die allgemeine Lebenszufriedenheit sollte hingegen möglicht hoch eingeschätzt werden, wobei hier die persönliche Meinung zu den Bereichen Gesundheit, Arbeit, Einkommen, Wohnung, und Umwelt abgefragt wird (ebd., S. 68)

Tabelle 4: Zweite Ebene der sozialen Nachhaltigkeit und deren Leitindikatoren

\begin{tabular}{|l|l|l|}
\hline Ebene sozialer Nachhaltigkeit & \multicolumn{1}{|c|}{ Indikator } \\
\hline Sozialressourcen & subjektiv & $\begin{array}{l}\text { Anteil der Bevölkerung mit Gefühlen der } \\
\text { Sinnlosigkeit und Einsamkeit }\end{array}$ \\
\cline { 2 - 3 } & objektiv & $\begin{array}{l}\text { Zeitaufwand für soziale, politische, ehrenamtliche } \\
\text { Aktivitäten }\end{array}$ \\
\hline
\end{tabular}

Die Sozialressourcen mit Indikatoren zu beschreiben, gestaltet sich sehr schwierig, da hier die Quantität sozialer Phänomene wenig über deren Qualität aussagt (ebd., S.69). Bei den Sozialressourcen geht es objektiv um die Messung der sozialen Beziehungen der Menschen zu ihrem Umfeld und ihr soziales Engagement, das in einigen Studien zeitlich erhoben wurde 
und quantifiziert werden konnte (ebd., S. 72). Der subjektive Indikator beschreibt die eigene Einschätzung der sozialen Einbindung in die Gesellschaft, die sich in dem Gefühl von Einsamkeit und einer empfundenen Macht- und Sinnlosigkeit der Bevölkerung erkennen lassen (ebd.). Um die Sozialressourcen zu nutzen und damit einen Beitrag zur sozialen Nachhaltigkeit zu leisten, sollte der objektive Indikator möglichst hoch, der subjektive Indikator dagegen möglichst gering eingeschätzt werden.

Tabelle 5: Dritte Ebene der sozialen Nachhaltigkeit und deren Leitindikatoren

\begin{tabular}{|l|l|l|}
\hline Ebene sozialer Nachhaltigkeit & & \multicolumn{1}{c|}{ Indikator } \\
\hline Chancengleichheit & Ressourcenverteilung & Gini-Koeffizient zur Einkommensverteilung \\
\cline { 2 - 3 } & Geschlecht & Gender Empowerment Measure (GEM) \\
\hline
\end{tabular}

In der oben stehenden Tabelle ist die Chancengleichheit als Ebene der sozialen Nachhaltigkeit mit den zugehörigen Leitindikatoren aufgeführt. Um die Ressourcenverteilung zu messen, gibt der Gini-Koeffizient Auskunft über die Einkommensverteilung innerhalb der Bevölkerung, wobei er als Maß für Ungleichheit gilt und je höher der Wert liegt, desto ungleicher ist die Verteilung (in diesem Fall) des Einkommens (Bundesministerium für Gesundheit und Soziale Sicherung, 2005a, Anhang, S. 13). Grundlagen für eine gerechte Chance am Leben teilhaben zu können, sind die gerechte Verteilung der verfügbaren Ressourcen und die Zugänglichkeit der Ressourcen. Dieser Koeffizient sollte möglich niedrig ausfallen, um Chancengleichheit zu gewährleisten (Empacher, Wehling, 2002, S.73 u. 75). Die Chancengleichheit bezieht sich neben den unterschiedlichen Gesellschaftsschichten auch auf die Gleichberechtigung der Geschlechter. Dieser Faktor wird über den GEM gemessen, der sich aus dem Anteil von Frauen im Parlament, in Verwaltungs- und Management Tätigkeiten und in technischen Berufen und dem geschlechtsspezifischen Anteil am Einkommen zusammensetzt (ebd., S. 60). Dieser GEM sollte dem Wert eins möglichst nahe kommen, um eine Chancengleichheit zwischen den Geschlechtern zu belegen.

Tabelle 6: Vierte Ebene der sozialen Nachhaltigkeit und deren Leitindikatoren

\begin{tabular}{|c|c|c|}
\hline Ebene sozialer Nachhaltigkeit & & Indikator \\
\hline Partizipation & objektiv & $\begin{array}{l}\text { Gewichteter Anteil derer, die sich an Wahlen und } \\
\text { nicht-institutionalisierten Partizipationsformen } \\
\text { beteiligen }\end{array}$ \\
\hline & subjektiv & Zufriedenheit mit politischer Partizipation \\
\hline
\end{tabular}

Die vierte Dimension von sozialer Nachhaltigkeit stellt die Partizipation dar. Hier wird als objektiver Indikator der Anteil der Bevölkerung, der sich an Wahlen beteiligt mit dem Anteil, der z.B. an Demonstrationen oder Unterschriftenaktionen teilnimmt, verwendet. Diese 
Teilnahme an gesellschaftlichen Entscheidungsprozessen, wobei die Beteiligung an nichtinstitutionalisierten Entscheidungsprozessen ein höheres persönliches Engagement erfordert als die Teilnahme an Wahlen, sollte möglichst hoch sein (ebd., S.75). Die subjektive Einschätzung der Zufriedenheit mit der eigenen politischen Partizipation sollte ebenfalls möglichst hoch eingeschätzt werden.

Zusammenfassend lässt sich festhalten, dass die soziale Nachhaltigkeit seitens der Soziologie durch die unterschiedlichen Ebenen beschrieben und mit Hilfe der Indikatoren erhoben werden kann. Die aufgeführten subjektiven Indikatoren können aus den Erhebungen des Sozio-ökonomischen Panels (SOEP) gewonnen werden.

Die gesellschaftlichen Belange der Nachhaltigkeit wurden vom damaligen Bundesministerium für Gesundheit und Soziale Sicherung in den Blickpunkt gerückt. So werden als wichtigste Komponenten der sozialen Dimension von Nachhaltigkeit folgende Punkte aufgeführt:

- „Verteilungsgerechtigkeit zwischen den Generationen (z.B. Altersversorgung), innerhalb der Generationen (sowohl national, z.B. zwischen arm und reich, als auch international, z.B. zwischen Industrie- und Entwicklungsländern).

- Dauerhaftigkeit (Sicherung des sozialen Friedens, Bildung, Sicherheit, Risikovermeidung).

- Integration (Annerkennung kultureller Unterschiede statt Ausgrenzung, Vernetzung).

- Partizipation (Mitsprache und Mitentscheidung der Betroffenen).“(Bundesministerium für Gesundheit und Soziale Sicherung, 2005b)

Die Dimensionen des Bundesministeriums decken sich zum großen Teil mit denen, die sich aus dem Vorschlag des Instituts für sozial-ökologische Forschung (s.o. Tabellen) ergeben. Werden die Aussagen und Indikatoren dieser Institutionen mit denen der Ministerkonferenz (MCPFE) verglichen, sollte an dieser Stelle festgehalten werden, dass der Forstsektor nicht in der Lage ist, die soziale Dimension von Nachhaltigkeit ausreichend zu definieren. Die forstlichen Indikatoren zur Beschreibung der sozialen Nachhaltigkeit sind von ökonomischen Ausgangslagen geprägt und konzentrieren sich nicht auf gesamtgesellschaftliche Aspekte bzw. Zusammenhänge. Dies ist aber angesichts der Situationen und Gegebenheiten der Gesellschaft innerhalb der urbanen Ballungszentren dringend erforderlich. Eine Neuausrichtung der Sozialfunktion des Waldes könnte die Integration von Problemgruppen entscheidend mitgestalten. Diese Aufgabe sollte von den Forstverwaltungen in urbanen Zentren wahrgenommen werden. 


\subsection{Akteure der Integrationsarbeit}

Die vielfältigen Aufgaben von Kommunen sind von einzelnen Fachveraltungen zu erfüllen. Eine allgemeine Definition von Verwaltung liefert Krott (2001, S. 96):

„Die Verwaltung ist jene öffentliche Institution, die auf der Grundlage von generellen gesetzlichen Normen Entscheidungen in speziellen Problemen trifft und die gefundenen Problemlösungen mit besonderen Maßnahmen durchsetzt."

Zur Durchführung der sozialen Aufgaben in Kommunen stehen Ämter, Einrichtungen und Dienste zur Verfügung. Die Ausgestaltung der Institutionen ist von der Organisationsfreiheit der Kommunen geprägt und orientiert sich an der örtlichen Bedarfslage. Dennoch haben sich weitgehend einheitliche Strukturen in den Kommunen herausgebildet (Bäcker et. al, 2000). Die wichtigsten Ämter für die Bereitstellung von sozialen Diensten und deren Hauptaufgaben sollen in der folgenden Tabelle zusammengefasst werden:

Tabelle 7: Kommunale Ämter der sozialen Dienste und deren Hauptaufgaben

\begin{tabular}{|c|c|}
\hline Ämter & Aufgaben \\
\hline Sozialämter & $\begin{array}{l}\text { - Abwicklung der Grundsicherung für Arbeitsuchende (Beratung, Information, } \\
\text { Geldleistungen und Sachleistungen zur Wiedereingliederung) } \\
\text { - Abwicklung der Sozialhilfe (Ermöglichung eines würdigen Lebens, umfasst u.a. Hilfe } \\
\text { zum Lebensunterhalt, Grundsicherung im Alter und bei Erwerbsminderung, Hilfen zur } \\
\text { Gesundheit, Eingliederungshilfen für behinderte Menschen, Hilfe zur Pflege) } \\
\text { - Hilfen für Wohnungslose } \\
\text { - Kriegsopferfürsorge } \\
\text { - Arbeit mit Spätaussiedlern und Asylbewerbern } \\
\text { - kommunale Sozialplanung }\end{array}$ \\
\hline Jugendämter & $\begin{array}{l}\text { - Aufgaben der Kinder- und Jugendhilfe ( u.a. Förderung und Entwicklung junger } \\
\text { Menschen, Förderung der Erziehung in der Familie, Förderung von Kindern in } \\
\text { Tageseinrichtungen und-pflege) }\end{array}$ \\
\hline Gesundheitsämter & $\begin{array}{l}\text { - gesundheitspolizeiliche Aufsicht und Überwachung } \\
\text { - ambulante psychiatrische Versorgung der Bevölkerung } \\
\text { - allgemeine Gesundheitsvorsorge und -beratung } \\
\text { - Kinder-, Jugend- und Müttergesundheitspflege } \\
\text { - Gesundheitsberichterstattung }\end{array}$ \\
\hline $\begin{array}{l}\text { (andere kommunale } \\
\text { Ämter) } \\
\text { - Wohnungsämter } \\
\text { - Versorgungsämter } \\
\text { - Ausgleichsämter }\end{array}$ & $\begin{array}{l}\text { - Wohnraumversorgung } \\
\text { - Behindertenhilfe } \\
\text { - Bearbeitung von Kriegs- und Vertreibungsschäden }\end{array}$ \\
\hline
\end{tabular}

Quellen: Bäcker et al. 2000, S. 364f.; Sozialgesetzbuch (SGB) II, VIII und XII

Die kommunalen Ämter des sozialen Dienstes sind demnach erste Ansprechpartner für Integrationsprojekte für unterschiedliche Personengruppen. 


\subsubsection{Aspekte der Integrationsarbeit}

Die Vereinigung der Sozialarbeiter in den USA hat 1990 in der Erklärung "Code of Ethics“ die Grundsätze ihrer Arbeit zusammengefasst (Edwards et al. 1995, zitiert nach Ginsberg, 1996, Übersetzung durch Verf.):

„Sozialarbeiter sollen das allgemeine Wohlergehen der Gesellschaft fördern.

1. Der/die Sozialarbeiter/in soll handeln, um Diskriminierung von Personen oder Gruppen aufgrund von Rasse, Hautfarbe, Geschlecht, sexueller Orientierung, Alter, Religion, nationaler Herkunft, Familienstand, politischer Überzeugung, geistiger oder körperlicher Behinderung, oder jeder anderen Vorliebe oder persönlichen Eigenschaft, Voraussetzung oder Zustand zu verhindern und abzuschaffen.

2. Der/die Sozialarbeiter/in soll handeln, um allen Menschen den Zugang zu den Ressourcen, Leistungen und Möglichkeiten zu sichern, die sie benötigen.

3. Der/die Sozialarbeiter/in soll handeln, um die Auswahl und Möglichkeiten für alle Menschen, mit besonderer Berücksichtigung benachteiligter und unterdrückter Gruppen und Personen zu erweitern.

4. Der/die Sozialarbeiter/in soll die Umstände fördern, die den Respekt für die Vielfalt der Kulturen bestärken, die die amerikanische Gesellschaft bilden.

5. Der/die Sozialarbeiter/in soll geeignete professionelle Leistungen für allgemeine Notfälle anbieten.

6. Der/die Sozialarbeiter/in soll für Änderungen in Politik und Gesetzgebung einstehen, um soziale Zustände zu verbessern und soziale Gerechtigkeit zu fördern.

7. Der/die Sozialarbeiter/in soll die informierte Partizipation der Öffentlichkeit bestärken durch Gestaltung der Sozialpolitik und Institutionen.

Diese Grundsätze gelten nicht nur für die amerikanische Gesellschaft bzw. die Sozialarbeiter in Amerika, sondern besitzen eine gewisse Allgemeingültigkeit. Denn auch in Europa und gerade in Deutschland finden sich z.B. große Unterschiede in der kulturellen Zusammensetzung der Gesellschaft und auch hier sollte sich für den Respekt gegenüber anderen Kulturen eingesetzt werden.

Mit dem Begriff der Integration wird zunächst die Eingliederung von Ausländern in die Gesellschaft verbunden. Esser (2001) beschreibt vier verschiedene Integrationstendenzen bei Menschen mit Migrationshintergrund.

Tabelle 8: Typen der Sozialintegration von Migranten (ebd., S. 19)

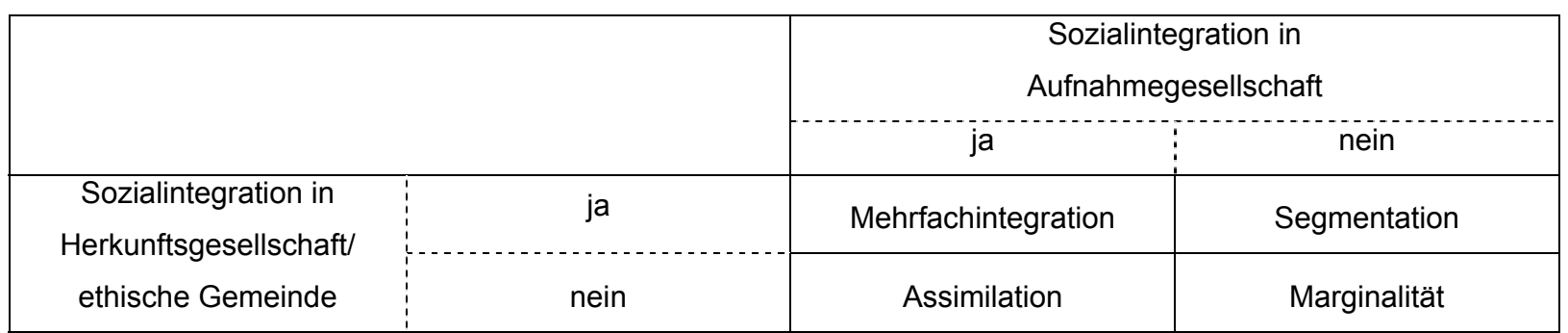

Die Sozialintegration von fremdethnischen Migranten kann sich auf drei gesellschaftliche Systeme beziehen, das Herkunftsland, das Aufnahmeland und die ethnische Gemeinde im Aufnahmeland (Esser, 2001, S.19). Die ethnischen Gemeinde und die Herkunftsgesellschaft 
sollen hier als ein System betrachtet werden, auf das sich die Integration bezieht. Es treten somit vier verschiedene Formen von Sozialintegration auf. Ist eine Person sowohl in die Aufnahmegesellschaft und die Herkunftsgesellschaft integriert, handelt es sich um eine Mehrfachintegration. Bei der ethnischen Segmentation ist die Person in die Gesellschaft des Herkunftslandes integriert bei gleichzeitiger Exklusion aus der Aufnahmegesellschaft. Als assimiliert kann eine Person bezeichnet werden, die in die Aufnahmegesellschaft sozial integriert ist, die eigenen ethnischen Bezüge aber aufgegeben hat. Ist eine Person in kein gesellschaftliches System integriert, handelt es sich um Marginalität bzw. um einen sozialintegrativen Ausschluss aus allen Bereichen (ebd., S. 20). Glatzer (2004) hat in einer Studie die Integration und Partizipation von 18-30 jährigen Personen mit türkischer, italienischer und deutscher Herkunft verglichen. Bei den jungen ausländischen Personen handelt es sich um die sog. ,Zweite Generation', d.h. deren Eltern bzw. Verwandte sind in den vergangenen Jahrzehnten zugewandert und sie selbst sind zum größten Teil in Deutschland geboren, ein geringerer Teil ist im Kindes- oder Jugendalter, noch weniger sind im jungen Erwachsenenalter nachgezogen (Glatzer, 2004, S. 102). Die Untersuchung zeigt ein große Ähnlichkeit der zweiten Generation mit ausländischer Herkunft und den deutschen jungen Erwachsenen im Hinblick auf sozialstrukturelle (Schulabschluss, Erwerbstätigkeit, Wohneigentum etc.), sozialkulturelle (deutsche Sprachkenntnisse, Fremdheitsgefühl, Akzeptanz eines deutschen bzw. ausländischen Ehepartner etc.) und soziopolitische (Vereinsmitgliedschaft, Cliquenzugehörigkeit, Demonstrationsteilnahme, Parteimitgliedschaft etc.) Aspekte. Dieses Ergebnis zeigt, dass eine Integration der ausländischen jungen Erwachsen durch wechselseitige Anpassung und Akzeptanz stattgefunden hat (ebd., S. 106). Die ausländischen jugendlichen Erwachsenen könnten somit als mehrfachintegriert bezeichnet werden, denn einige ethnische Gruppen pflegen die Traditionen der Herkunftsgesellschaft, nehmen aber auch gleichzeitig am gesellschaftlichen Leben der Aufnahmegesellschaft teil. Die soziale Integration bezieht sich aber nicht allein auf die Akzeptanz und Toleranz von Menschen mit ausländischer Herkunft, sondern umfasst einen weiten Rahmen von Personengruppen. Im Folgenden sollen die Grundsätze der Sozialen Arbeit allgemein dargestellt werden.

\subsubsection{Soziale Dienste}

Soziale Probleme von Menschen in einer Gesellschaft können z. T. nicht durch die Breitstellung von mehr Einkommen gelöst oder gemindert werden. In bestimmten Lebenslagen kann Menschen durch ein höheres Einkommen kurzfristig geholfen werden, aber die Lebensqualität hängt auch von den personengebundenen Hilfesystemen ab (Bäcker 
et al., 2000, S. 332). Der Hilfebedarf der Betroffenen kann nicht nur in außergewöhnlichen sondern auch in typischen Lebenslagen existieren. So sind z.B. Kinder auf die Hilfe Anderer angewiesen, um sich zu entwickeln. Die nachfolgende Tabelle soll einen Überblick der Hilfeund Unterstützungsleistungen geben (nach Bäcker et al., 2000, S. 333):

Tabelle 9: Ausgewählte Anlässe, Zielgruppen und Handlungstypen sozialer Hilfs- und Unterstützungsleistungen

\begin{tabular}{|l|l|l|}
\hline \multicolumn{1}{|c|}{ Anlässe } & \multicolumn{1}{|c|}{ Zielgruppen } & \multicolumn{1}{c|}{ Handlungsformen } \\
\hline - Versorgungs- und Betreuungsbedarf & - Kinder und Jugendliche & - Unterrichten und Erziehen \\
bei Kindern & - Ältere & - Beraten und Informieren \\
- Krankheit, Behinderung, & - Kranke & - Betreuen und Versorgen \\
Pflegebedürftigkeit & - Behinderte & - Behandeln, Pflegen, \\
- Bildungs-, Beratungs- und & - Pflegebedürftige & Rehabilitieren \\
Erziehungsbedarf & - Migranten und Flüchtlinge & - Hilfe bei häuslichen Verrichtungen \\
- Kontaktarmut, Ausgrenzung, Isolation & & - Emotionale und psychosoziale \\
- Hilfsbedürftigkeit bei der alltäglichen & & Zuwendung \\
Lebensführung & & \\
- Erziehungs- und & & \\
Partnerschaftsprobleme & & \\
- Sucht & & \\
- Seelische Notlagen & & \\
- Soziale Gefährdung & & \\
\hline
\end{tabular}

Als Soziale Dienste werden diejenigen Hilfeleistungen angesehen, die professionell, organisiert und entgeltlich (Nutzer zahlt für die Leistung) erbracht werden (ebd.). Die sozialen Dienste unterscheiden sich von anderen erwerbsförmigen personenbezogenen Diensten durch die Existenz eines sozialen Problems bzw. dessen, was von der Gesellschaft als soziales Problem angesehen und anerkannt wird (ebd.). Die Dauer der Nutzung sozialer Dienste hängt von der Lebenslage ab, in der sich der Hilfebedürftige befindet. So können sie längerfristig bis ständig von Nöten sein, z.B. Hilfen für Behinderte, Pflegebedürftige oder die Betreuung von Kindern. Kurzfristige oder sporadische Hilfen sind z.B. bei akuten Krankheitsfällen oder spezifischen Beratungen notwendig. Z.B. bei Krankheiten oder Unfällen kann der Hilfebedarf unerwartet und plötzlich auftreten oder er kann sich schleichend entwickeln, z.B. bei Pflegebedürftigen oder absehbar auftreten, wie z.B. die Betreuung von Kindern (ebd., S. 334). Charakteristika sozialer Dienste sind weiterhin, dass sie unterstützend, beratend und helfend wirken (s. Tab. 6). Sie können als personenorientiert bezeichnet werden und es gilt das „uno-actu-Prinzip“, was bedeutet, das Dienstleistung bzw. Produktion und Konsumption zeitlich und räumlich zusammenfallen, da der Dienst an und mit Personen vollzogen wird (ebd.). Eine wichtige Voraussetzung für ein qualitativ gutes Ergebnis sozialer Dienste ist die Kooperationswilligkeit und -fähigkeit des 
Leistungsnehmers, denn nur mit dessen aktiver Beteiligung kann dies gelingen. Ein weiteres Merkmal der sozialen Dienste ist die Zuteilung zu den Erfahrungsgütern, denn erst nach Erbringung der Leistung kann die Qualität beurteilt und geprüft werden, z.B. die Bewertung einer Pflegeleistung (ebd.). Zudem handelt es sich bei den sozialen Diensten um Vertrauensgüter. Der Leistungsnehmer muss darauf vertrauen, dass er die für ihn richtige Leistung erhält. Bei mangelnder Sachkenntnis des Leistungsnehmers, welche Leistungen die erfolgsversprechenden sind, begibt er sich in ein Abhängigkeitsverhältnis zu dem Leistungsanbieter, der berät und Einzelleistungen vorschlägt (ebd.). Soziale Dienste werden in der Regel nicht zu Marktpreisen angeboten und nachgefragt, was ebenfalls ein Charakteristikum darstellt. Die Dienste werden größtenteils öffentlich finanziert und als Sachleistungen erbracht und sind somit dem Markt- und Wettbewerbsprozess entzogen (ebd., S. 335). Gründe für die öffentliche Finanzierung liegen in der fehlenden unmittelbaren Qualitätsprüfung durch den Leistungsempfänger und weiterhin sollen die Probleme bzw. Bedarfe gelöst und gedeckt werden, ohne von der individuellen Zahlungsbereitschaft und Zahlungsfähigkeit der betroffenen Personen abhängig zu sein (ebd.). Die sozialen Dienste werden den Zielgruppen bzw. dem Klientel entsprechend eingesetzt. So gelten für die Beratung von Menschen mit Behinderungen andere Methoden/Richtlinien/Maßstäbe und Ziele als in der Beratung von Suchtkranken. Die Angebote und Dienste für Menschen mit Behinderungen erstrecken sich meist auf alle Lebensphasen. Beginnend mit der ,Frühförderung' in der Kindheit, über die schulische Betreuung und Bildung bis hin zur Arbeit oder alternativen Beschäftigungsmöglichkeiten. Das übergeordnete Ziel der Maßnahmen besteht darin, den Menschen mit Behinderungen eine Teilnahme am Leben in der Gemeinschaft zu ermöglichen, eine Behinderung bzw. deren Folgen zu vermeiden oder zu lindern und die Förderung einer selbständigen Lebensführung (Metzler u. Wacker, 2005, S.132). 


\subsubsection{Suchtarbeit}

Bevor die Maßnahmen zur Bekämpfung von Sucht beschrieben werden, soll zunächst der Begriff der Sucht eingegrenzt werden. Von Wolffersdorff (2005, S. 324) definiert Sucht wie folgt:

„Im Kontext der heutigen Drogenpolitik wird unter Sucht vor allem der zwanghafte Gebrauch bzw. Mißbrauch von Substanzen (Suchtgiften), das Bedürfnis nach Steigerung der Dosis und das Moment des Nicht-mehr-Aufhörenkönnens verstanden.“

Süchtige Verhaltensweisen zeichnen sich weiterhin dadurch aus, dass die Betroffenen die Kontrolle über ihren Willen und die Verantwortung für das eigene Tun verlieren. Der Wunsch nach dem Wohlbefinden, welches die Substanz auslöst und die erfahrene Euphorie noch zu steigern, entwickelt sich zu einem körperlichen Verlangen, dem sich die Betroffenen sehr schwer entziehen können. Die Sucht ist als psychologischer Prozess zu verstehen, der durch die Reaktion des Körpers auf eine bestimmte Substanz ausgelöst wird, also nicht um einen Vorgang, den die Substanz bzw. das ,Gift' mechanisch im Körper verursacht (ebd.). Die Drogenhilfe bzw. die Angebote der Drogenhilfe richten sich flexibel nach den Bedürfnissen der Leistungsnehmer (Jungblut, 2005). Unterschieden wird in der Drogenhilfe zwischen Präventiv- und suchtbegleitenden Maßnahmen. Die Suchtprävention zielt darauf ab, dass Situationen im Zusammenleben ohne den Rückgriff auf Drogen bewältigt werden können. Die Suchtbegleitende Drogenhilfe bedeutet:

- „Hilfen nicht an Auflagen wie Abstinenz und Therapiebereitschaft zu binden.

- Psychosoziale, medizinische, hygienische und pädagogische Hilfen so zu organisieren, dass sie der Verelendung von Drogenabhängigen entgegenarbeiten, dazu gehören auch die Beratung und Betreuung von Substituierten sowie die Beratung von Konsumenten in Hinblick auf Substitutionsmaßnahmen.

- Diese Hilfen in den alltäglichen Lebensvollzügen von Drogenabhängigen organisieren durch das Bereitstellen eines vernetzten Hilfeangebotes, dazu gehören Kontaktläden und Streetwork ebenso wie Notschlafstellen. Entzugsmöglichkeiten durch Krankenhäuser oder eigene, dafür eingerichtete medizinischen Ambulatorien sowie Wohngemeinschaften für Abhängige bzw. Substituierte, Beratungsstellen und Präventionsfachstellen." (Jungblut, 2005, S. 292)

In der Drogenpolitik werden drei Modelle unterschieden, dass Modell der Schadensminimierung, das therapeutische Modell und das Modell der Kontrolle. Im Folgenden soll eine Tabelle Aufschluss über die wichtigsten Merkmale der drei Modelle geben: 
Tabelle 10: Wichtigste Merkmale der drei Modelle der Drogenpolitik (vgl. Jungblut, 2005, S. 285)

\begin{tabular}{|c|c|c|c|}
\hline Dimension & $\begin{array}{c}\text { Modell der Schadens- } \\
\text { minimierung }\end{array}$ & Therapeutisches Modell & Modell der Kontrolle \\
\hline Hauptziel & $\begin{array}{l}\text { - Minimalisierung der durch } \\
\text { Sucht erzeugten Probleme }\end{array}$ & $\begin{array}{l}\text { - Heilung der } \\
\text { Drogensüchtigen }\end{array}$ & - Drogenfreie Gesellschaft \\
\hline $\begin{array}{l}\text { Fundamentaler } \\
\text { Wert }\end{array}$ & - individuelle Integrität & - gesellschaftliche Integrität & - Integrität der Gemeinschaft \\
\hline Prävention & $\begin{array}{l}\text { Ziel: Verantwortung } \\
\text { - pluralistische Aussagen } \\
\text { - differenzierte Prävention } \\
\text { - Schadensminimierung }\end{array}$ & $\begin{array}{l}\text { Ziel: Abschreckung } \\
\text { - „technische“ Aussagen } \\
\text { - wenig Prävention }\end{array}$ & $\begin{array}{l}\text { Ziel: Abschreckung } \\
\text { - moralisierende Prävention } \\
\text { - große Verbreitung der } \\
\text { präventiven Aussagen }\end{array}$ \\
\hline Betreuung & $\begin{array}{l}\text { - Begleitung (Stabilisierung) } \\
\text { - diversifiziertes } \\
\text { Therapieangebot } \\
\text { - Wiedereingliederung } \\
\text { - substitutive Medikament } \\
\text { - streetwork }\end{array}$ & $\begin{array}{l}\text { - Notfalldienste } \\
\text { - Betreuungs-/ } \\
\text { Pflegeangebote } \\
\text { - individualisierte Behandlung } \\
\text { - minimale Wiederein- } \\
\text { gliederungsangebote } \\
\text { - Substitution marginal }\end{array}$ & $\begin{array}{l}\text { - abstinenzorientierte } \\
\text { Therapie } \\
\text { - Wiedereingliederung } \\
\text { - Substitution marginal } \\
\text { - streetwork }\end{array}$ \\
\hline Kontrolle & $\begin{array}{l}\text { - Repression des } \\
\text { organisierten Drogenhandels } \\
\text { - Toleranz gegenüber } \\
\text { Kleinhandel und Konsum } \\
\text { - Alternative Therapie statt } \\
\text { Gefängnis }\end{array}$ & $\begin{array}{l}\text { - Repression des } \\
\text { organisierten Drogenhandels } \\
\text { - Toleranz gegenüber } \\
\text { Konsum } \\
\text { - Alternative Therapie statt } \\
\text { Gefängnis }\end{array}$ & $\begin{array}{l}\text { - Repression des Drogen- } \\
\text { handels und -konsums } \\
\text { - spezielle Gefängnisse } \\
\text { - Alternative Therapie statt } \\
\text { Gefängnis }\end{array}$ \\
\hline Koordination & $\begin{array}{l}\text { - intensive Koordination } \\
\text { zwischen versch. Bereichen } \\
\text { - institutionalisierte } \\
\text { Konfliktlösungsinstanzen }\end{array}$ & $\begin{array}{l}\text { - Koordination innerhalb des } \\
\text { therapeutischen Bereiches }\end{array}$ & $\begin{array}{l}\text { - starke Integration und } \\
\text { Interpenetration der versch. } \\
\text { Bereiche }\end{array}$ \\
\hline Politikstil & $\begin{array}{l}\text { - Verantwortung des } \\
\text { Einzelnen } \\
\text { - trial and error - Politik } \\
\text { - experimentierender Staat } \\
\text { "Anreizstaat" } \\
\text { - Beteiligung der privaten } \\
\text { Organisationen }\end{array}$ & $\begin{array}{l}\text { - staatliche Verantwortung } \\
\text { gegenüber den „Kranken“ } \\
\text { - technokratischer Staat } \\
\text { - lokale Tradition vs. } \\
\text { Zentralstaat } \\
\text { - subsidiäre Rolle der privaten } \\
\text { Organisationen }\end{array}$ & $\begin{array}{l}\text { - staatliche und zivile } \\
\text { Verantwortung gegenüber } \\
\text { den Süchtigen } \\
\text { - Vorsorgestaat } \\
\text { - moralisierende Politik } \\
\text { - Beteiligung der privaten } \\
\text { Organisationen }\end{array}$ \\
\hline Hauptstärken & $\begin{array}{l}\text { - pluralistische und flexible } \\
\text { Antworten } \\
\text { - Integration Staat / } \\
\text { Gesellschaft }\end{array}$ & $\begin{array}{l}\text { - kohärente Therapieketten } \\
\text { - Respekt der individuellen } \\
\text { Autonomie }\end{array}$ & $\begin{array}{l}\text { - soziale Problematik } \\
\text { - keine größeren Konflikte }\end{array}$ \\
\hline
\end{tabular}

Diese Auflistung zeigt, wie komplex und vielseitig die Hilfesysteme in der Drogenpolitik aufgebaut sind. Hieran wird deutlich, dass nur mit Hilfe von professionellem Fachpersonal 
die Ziele der einzelnen Modelle erreicht werden und Hilfeprogramme umgesetzt werden können.

\subsubsection{Wohnungslosenhilfe}

Im Folgenden sollen die Maßnahmen und Strukturen der Sozialarbeit in Bezug auf Obdachlose dargestellt werden. Zunächst ist es wichtig zu erwähnen, dass es keine einheitliche, von allen Fachleuten anerkannte Definition zur Beschreibung von Menschen gibt, die nicht über hinreichenden Wohnraum verfügen (Wolf, 2005, S. 1292). Der Deutsche Städtetag hat Ende der 80iger Jahre den Begriff des Wohnungsnotfalls in die Diskussion eingebracht. So werden diejenigen Menschen als Wohnungsnotfall beschrieben, die unmittelbar von Wohnungslosigkeit bedroht sind, diejenigen, die aktuell von Wohnungslosigkeit betroffen sind und die Personen, die in unzumutbaren Wohnverhältnissen leben (vgl. ebd.). Die Angebote und Maßnahmen der Obdachlosenhilfe bzw. der Obdachlosenarbeit sind vielfältig und haben im Laufe der Geschichte eine starke Wandlung erfahren. So wurde mit Beginn der Industrialisierung eine vermehrte Obdachlosigkeit und Wohnungslosigkeit festgestellt, da Unterkünfte und Wohnung als Ware gehandelt wurden und es entstand die sogenannte "Wanderarmut". Von dieser waren Menschen betroffen, die der Arbeit „hinterher wandern“ mussten. In der Hilfe wurde hier ein Schwerpunkt in der Verbesserung der Situation für die Kinder gesetzt, wodurch die „Vererbung“ von Obdachlosigkeit verhindert werden sollte. Die Erwachsenen wurden in abgestuften Unterkünften untergebracht und sollten nach einer Bewährungszeit in normale Wohnverhältnisse ,aufsteigen' (ebd., S. 1294). Ziel der heutigen Wohnungslosenhilfe ist es, den Personengruppen, die auf dem Wohnungsmarkt nur eingeschränkte oder gar keine Chancen haben, in verschiedenen Formen Wohnraum zur Verfügung zu stellen (ebd., S. 1298). Dies kann durch Kooperationen zwischen der Sozialarbeit und den Wohnungsunternehmen geschehen. Der Bereich der Wohnungslosenhilfe ist stark von Einzelfällen geprägt, für die ein ganz individuelles Hilfekonzept erarbeitet werden muss.

\subsubsection{Jugendarbeit}

Ein ähnlich komplexes Gebiet der Sozialarbeit stellt die Jugendarbeit dar. Die Ausführungen in der vorliegenden Arbeit können nur einen kurzen Einblick in die Struktur und die Ziele dieses Bereiches geben. Die Kinder- und Jugendarbeit wird wie folgt zusammengefasst: 


\begin{abstract}
„Kinder- und Jugendarbeit umfasst alle außerschulischen und nicht ausschließlich berufsbildenden, mehr oder weniger pädagogisch gerahmten, nicht kommerziellen erlebnis- und erfahrungsbezogenen Sozialisationsfelder von freien und öffentlichen Trägern, Initiativen und Arbeitsgemeinschaften, in denen Kinder und Jugendliche ab dem Schulalter selbständig, mit Unterstützung oder in Begleitung von ehrenamtlichen und/oder beruflichen MitarbeiterInnen individuell oder in Gleichaltrigengruppen zum Zweck der Freizeit, Bildung und Erholung einmalig, sporadisch, über einen turnusmäßigen Zeitraum oder für eine längere, zusammenhängende Dauer zusammen kommen können. (Thole, 1995; zitiert nach Hafeneger u. Schröder, S. 840).
\end{abstract}

Allgemein kann als Ziel der Jugendarbeit formuliert werden, dass diese eine Unterstützung der Jugendlichen bei der Selbstwerdung leisten will. Hierbei sollen keine Vorgaben über die Entwicklungsziele gemacht werden, aber dennoch soll gegenseitiger Respekt und Annerkennung zwischen Jugendlichen und Pädagogen/innen herrschen (Hafeneger $u$. Schröder, 2005, S. 846). Als weiteres allgemeines Ziel der Jugendarbeit ist zu nennen, dass die Jugendlichen in dem Prozess des Erwachsenwerdens unterstützt und gefördert werden und in der Nachdenklichkeit und Einsicht gestärkt werden sollen. Die Erwachsenen treten hierbei als Begleiter mit der Bereitschaft zur Konfliktaustragung, Aufzeigen von Grenzen und Aufgabenverteilung auf. So sollen die Jugendlichen einen respektvollen zwischenmenschlichen Umgang erlernen, der nicht von Aggressionen geprägt ist (vgl. ebd., S. 846). Die praktische Jugendarbeit wird in unterschiedlichen Formen angeboten, die im Folgenden kurz beschrieben werden sollen.

Gruppenarbeit: In der Gruppenarbeit liegt das Hauptaugenmerk im Erlernen und Erfahren in der Gemeinschaft. Die Gruppen können ganz unterschiedlich organisiert sein, z.B. als weitgehend selbstorganisierte Gruppe bis hin zu trägergeleiteten Gruppen, die einen Rahmen für die Arbeit vorgeben. Einen neuen Stellenwert erhält die Cliquenarbeit als Form der aufsuchenden Jugendarbeit, da Jugendliche meist in Cliquen integriert und sie dort am besten erreicht werden können.

Offene Arbeit: Die offene Arbeit kann mit dem Begriff ,offener Betrieb' gleichgestellt werden. In den Jugendhäusern, -zentren und -einrichtungen stehen den Jugendlichen Räume zu bestimmten Zeiten und unter Beachtung von Regeln zur Verfügung. In den meisten Fälle können sie diese mitbestimmen, mitgestalten und die Räume selbst verwalten. Im Vordergrund steht hierbei die Geselligkeit mit Gleichaltrigen. Der offene Betrieb ist ebenfalls eine Chance für die Mitarbeiter/innen, die Jugendlichen und die Cliquen kennen zu lernen, ihnen Beratung und Hilfe anzubieten und gezielte Veranstaltungen zu bewerben. Zu erwähnen ist hierbei noch, dass die offene Jugendarbeit allen Jugendlichen aus allen sozialen Schichten offen steht und auch aus dieser Vielfalt heraus lebt.

Seminare und Freizeiten: Diese Form der Jugendarbeit findet über einen gewissen Zeitraum, wie z.B. ein Wochenende oder eine Woche statt. Jugendliche kommen hier meist in Bildungseinrichtungen zusammen und behandeln für die Dauer ein bestimmtes Thema, z.B. abwechselungsreiche Gestaltung der Freizeit. Die Seminare oder Freizeiten werden in der 
Regel frei ausgeschrieben oder richten sich an bestimmten Gruppen (politische Jugendorganisationen, kulturelle Jugendgruppen etc.)

Projektarbeit: In dieser Arbeitsform sollen die Jugendlichen handlungsorientiert und ganzheitlich ein bestimmtes Thema erlernen und bearbeiten. Die Besonderheit liegt in der gemeinsamen Planung, Durchführung und Präsentation des Projektes aller Teilnehmer. Die Methode, die vor allem als Alternative zur traditionellen schulischen Lernsituation entwickelt wurde, ist aus dem Bereich der Jugendarbeit nicht mehr wegzudenken. (vgl. Hafeneger u. Schröder, 2005, S. 848-849). Festzuhalten bleibt hier, dass die Jugendarbeit als Hilfestellung für Jugendliche bei der Bewältigung von Problemen im Erwachsenwerden und dem Selbstfindungsprozess zu sehen ist.

\subsubsection{Arbeitslosenhilfe}

Die Sozialarbeit setzt sich ebenfalls mit der Bewältigung der Probleme von Arbeitslosen bzw. Langzeitarbeitslosen auseinander. Einen Schwerpunkt stellt hierbei die Arbeitsförderung dar. Die Arbeitsförderung soll allgemein dazu beitragen, einen hohen Beschäftigungsgrad zu gewährleisten und die Beschäftigungsstruktur ständig zu verbessern. Die Leistungen der Arbeitsförderung sollen folgende Ziele verfolgen:

1. „den Ausgleich von Angebot und Nachfrage auf dem Ausbildungs- und Arbeitsmarkt unterstützen,

2. die zügige Besetzung offener Stellen ermöglichen,

3. die individuelle Beschäftigungsfähigkeit durch den Erhalt und Ausbau von Kenntnissen, Fertigkeiten sowie Fähigkeiten fördern,

4. unterwertiger Beschäftigung entgegenwirken und

5. zu einer Weiterentwicklung der regionalen Beschäftigungs- und Infrastruktur beitragen (Sozialgesetzbuch (SGB) III, Arbeitsförderung, §1(2))

Das Gesetz unterscheidet unter den Leistungsempfängern zwischen „Arbeitslosen', ,von Arbeitslosigkeit bedrohte Arbeitnehmer' und ,Langzeitarbeitslosen'. In die Kategorie der Arbeitslosen fallen diejenigen Personen, die vorübergehend nicht in einem Beschäftigungsverhältnis stehen, eine versicherungspflichtige Arbeit suchen und somit den Vermittlungen der Agentur für Arbeit zur Verfügung stehen und dort arbeitslos gemeldet sind (SGB III, §16). Von Arbeitslosigkeit bedroht sind Personen, die versicherungspflichtig beschäftigt sind, aber in der nächsten Zukunft mit der Beendigung der Beschäftigung rechnen müssen und daraufhin voraussichtlich arbeitslos werden (SGB III, §17). Als langzeitarbeitslos gelten Personen, die ein Jahr und länger arbeitslos sind (SGB III, §18). Um den o.g. Personengruppen bei der Arbeitsplatzerhaltung und der Arbeitsplatzsuche bzw. Eingliederung in den Arbeitsmarkt zu unterstützen, sieht die aktive Arbeitsförderung folgende Leistungen vor: 
- „Berufsberatung sowie Ausbildungs- und Arbeitsvermittlung und diese unterstützende Leistungen,

- Maßnahmen der Eignungsfeststellung, Trainingsmaßnahmen zur Verbesserung der Eingliederungsaussichten,

- Mobilitätshilfen zur Aufnahme einer Beschäftigung,

- Gründungszuschuss zur Aufnahme einer selbstständigen Tätigkeit,

- Berufsausbildungsbeihilfe während einer beruflichen Ausbildung oder einer berufsvorbereitenden Bildungsmaßnahme,

- Übernahme der Weiterbildungskosten der während der Teilnahme an einer beruflichen Weiterbildung,

- allgemeine und als behinderte Menschen zusätzlich besondere Leistungen zur Teilhabe am Arbeitsleben und diese ergänzende Leistungen nach diesem und dem Neunten Buch, insbesondere Ausbildungsgeld, Übernahme der Teilnahmekosten und Übergangsgeld

- Kurarbeitergeld bei Arbeitsausfall,

- Wintergeld,

- Transferleistungen." (SGB III, § 3(1))

Zu den aktiven Leistungen der Arbeitsförderung zählen nach dem vierten Abschnitt dieses Paragraphen nicht die Zahlung von Arbeitslosengeld, Teilarbeitslosengeld und Insolvenzgeld. Neben den Arbeitnehmern können auch die Arbeitgeber die Leistungen der Arbeitsförderung beziehen. Hierzu zählen u. a. die Arbeitsmarktberatung, Zuschüsse zur Ausbildungsvergütung, Erstattung von Praktikumsvergütung, Zuschüsse zur Weiterbildung beschäftigter Mitarbeiter und auch die Zuschüsse bei Arbeitsentgelten zur Eingliederung von leistungsgeminderten Arbeitnehmern (SGB III, §3(2)). Die Maßnahmen und Leistungen auf Arbeitnehmer- und -geberseite haben allgemein die Vermeidung von Arbeitslosigkeit bzw. Langzeitarbeitslosigkeit zum Ziel. Die Auswahl der Leistungen für den Einzelfall hängt von den Fähigkeiten der zu fördernden Person ab, von der Aufnahmefähigkeit des Arbeitsmarktes und dem arbeitsmarktpolitischen Handlungsbedarf (SGB III, §7). Zur Umsetzung der Arbeitsförderung ist die Agentur für Arbeit angehalten, mit den Trägern der Maßnahmen auf Gemeinde-, Kreis- oder Bezirksebene zusammen zu arbeiten (SGB III, 9(3)). Hierzu stehen die Beschäftigungsförderungsorganisationen, wie z.B. KiK e.V. in Göttingen (Gemeindeebene), AGIL mbH in Kassel (Kreisebene), passage gGmbH (Stadtteil) in Hamburg als Partner zur Verfügung. Diese Organisationen beschäftigen vor allem die Klientel der Langzeitarbeitslosen und versuchen mit geeigneten Maßnahmen eine Wiedereingliederung in den Arbeitsmarkt zu erleichtern. Einige der Maßnahmen sind aus dem Konzept der Gemeinnützigen Beschäftigung entnommen, dem zwei Ideen zugrunde liegen:

„Alle gering qualifizierten Transferempfänger sollen die Möglichkeit haben, durch eigene Arbeit einen Beitrag zu ihrem Lebensunterhalt zu erwirtschaften und sich wieder in die Gesellschaft zu integrieren. Und das brachliegende Arbeitspotential soll genutzt werden, um die Träger von Sozialleistungen zu entlasten und wirtschaftliches Wachstum zu erleichtern. (Eeckhoff u. Roth, 2002, S.70)

Die Gemeinnützige Beschäftigung ist durch einige Merkmale charakterisiert, die im Folgenden beschrieben werden sollen. Nehmen Personen an einer Maßnahme zur 
gemeinnützigen Beschäftigung teil, beziehen sie weiterhin Sozial- und Arbeitslosenhilfe und scheiden erst mit dem Abschluss eines normalen, nicht subventionierten Arbeitsvertrages aus dem Programm aus. Während der Beschäftigungszeit erhalten sie keinen zusätzlichen Lohn, sondern eine Aufwandsentschädigung, welche die Kosten der Beschäftigung deckt, wie z.B. Fahrtkosten oder Kosten für spezielle Arbeitskleidung. Die Gemeinnützige Arbeit wird direkt in Kommunen oder gegen eine Gebühr bei privaten Unternehmen geleistet. Die Maßnahmenträger sind für alle Organisations- und Materialkosten sowie die Aufwandsentschädigungen verantwortlich. Es entstehen dabei keine Subventionsleistungen. Ein weiteres Merkmal der Gemeinnützigen Arbeit ist, dass die Teilnehmer ihren Fähigkeiten entsprechend eingesetzt werden und Weiter- und Fortbildungsmöglichkeiten nutzen können. Hierbei soll keine künstliche Beschäftigungssituation angestrebt werden. Bis zum Abschluss eines Arbeitsvertrages und damit dem Verzicht auf die Transferzahlungen kommen entweder die direkte Arbeitsleistung oder der Gegenwert der Leistung aus der Gemeinnützigen Arbeit dem Träger der Transferzahlung zugute. Zudem steht die Gemeinnützige Arbeit allen Hilfeempfänger offen und ist keiner zeitlichen Begrenzung unterlegen (im Gegensatz zu z.B. den Arbeitsbeschaffungsmaßnahmen) (Eeckhoff, Roth, 2002, S. 70-71). Zudem wurde das Kriterium der Zusätzlichkeit eingeführt. Die Tätigkeiten, die sonst nicht, nicht im gleichen Umfang oder erst zu einem späteren Zeitpunkt durchgeführt werden könnten, gelten als zusätzlich. Im Vordergrund dieser Überlegung steht die Verdrängung regulärer Arbeitsplätze durch die Gemeinnützigen Beschäftigungen (ebd., S. 84-85). Eine weitere Möglichkeit, gering qualifizierte Personen ohne Arbeitsverhältnis wieder in den Arbeitsmarkt zu integrieren, sind die sog. Maßnahmen mit Mehraufwandsentschädigung (MAE). Hier erhalten die Teilnehmer einer Maßnahme weiterhin die Hilfe zum Lebensunterhalt und für den Mehraufwand, den sie durch eine angebotene Arbeit haben, wird eine Entschädigung gezahlt. Auch hier besteht das Kriterium der Zusätzlichkeit. Die unterschiedlichen Programme zur Arbeitsförderung haben das gemeinsame Ziel der Wiedereingliederung von Arbeitslosen bzw. Langzeitarbeitslosen in den Arbeitsmarkt. Die aus den Programmen entstehenden Maßnahmen werden auf dieses Ziel abgestimmt und an deren Ende sollte zumindest eine Qualifizierung stehen, welche die Bewerbungschancen der Personen auf dem Arbeitsmarkt erhöhen. 


\subsubsection{Strafvollzug}

Einen weiteren Aufgabenbereich der Sozialarbeit stellt der Strafvollzug (Jugend- und Erwachsenenstrafvollzug) dar. Die allgemeine Aufgabe der Sozialarbeit ist die Verhinderung und Vermeidung von schädlichen Folgen des Strafvollzugs, um eine Resozialisierung schon während des Vollzugs zu fördern. Die Hilfe der Sozialarbeit richtet sich sowohl an die Gefangenen als auch an die Angehörigen (Mührel, 2005, S. 1844). Die Resozialisierung steht im Strafvollzug als erreichbares Ziel stets im Vordergrund und ist im Gesetzt wie folgt definiert:

„Im Vollzug der Freiheitsstrafe soll der Gefangene fähig werden, künftig in sozialer Verantwortung ein Leben ohne Straftaten zu führen (Vollzugsziel). Der Vollzug der Freiheitsstrafe dient auch dem Schutz der Allgemeinheit vor weiteren Straftaten." ( $\$ 2$ StVollzG)

Der Vollzug sollte weiterhin so gestaltet sein, dass er dem Gefangenen hilft, sich in das anschließende Leben in Freiheit einzugliedern (§3 (3) StVollzG). Die Sozialarbeit erstreckt sich hierbei über alle Phasen des Strafvollzugs, von der Aufnahme der Haft bis zur Entlassung. Die Hilfe zur Entlassung und direkt im Anschluss an die Haft ist von entscheidender Bedeutung. Das Gesetz sieht folgende Hilfe zur Entlassung vor:
„Um die Entlassung vorzubereiten, ist der Gefangene bei der Ordnung seiner persönlichen, wirtschaftlichen und sozialen Angelegenheiten zu beraten. Die Beratung erstreckt sich auch auf die Benennung der für Sozialleistungen zuständigen Stellen. Dem Gefangenen ist zu helfen, Arbeit, Unterkunft und persönlichen Beistand für die Zeit nach der Entlassung zu finden." (§74 StVollzG)

Die Entlassung stellt für die Betroffenen und deren Angehörigen oftmals eine große Herausforderung dar, die sie allein nicht bewältigen können. Die Entlassungshilfe der Sozialarbeit in der Anstalt wir meist in enger Zusammenarbeit mit den staatlichen und freien Trägern der Straffälligenhilfe durchgeführt (Mührel, 2005, S. 1846). Es soll hierbei konkret Hilfe zur Suche einer Unterkunft und deren Finanzierung, einer Beschäftigung, die Vollständigkeit der Papiere (Ausweise, Sozialversicherungen etc.), die Beratung bzgl. wirtschaftlicher (z.B. Schuldnerberatung) und persönlicher (z.B. Bewältigungsstrategien) Probleme (ebd.) geleistet werden. Alle Hilfsmaßnahmen während und direkt im Anschluss an den Strafvollzug zielen auf die Wiedereingliederung der Personen in die Gesellschaft ab und sollen den Weg in ein straffreies Leben ebnen. 


\section{Forschungsansatz}

Das Forschungsprojekt setzt sich mit der Frage auseinander, wie die kommunalen Forstverwaltungen ihr Handeln umstellen müssen, um zur Integration von sozialen Problemund Randgruppen beizutragen. Zunächst kann festgehalten werden, dass es eine Reihe von Institutionen gibt, die sich mit der Aufgabe der Integration beschäftigen und diese mit ihren Instrumenten positiv beeinflussen. Generell sollten zwei Formen der Handlungen zur Integration unterschieden werden. Verwaltungen sind zunächst durch die hierarchischrestriktive Aufgabenerledigung gekennzeichnet, d.h. die untergeordnete Stelle erledigt die Aufgaben, die ihr von übergeordneter Stelle aufgetragen werden. Ein hierarchisches Aufgabenverständnis kann zu hierarchisch-restriktiven Handlungen führen, die sich auch auf die Integration übertragen lassen. Ein Beispiel für eine die restriktive Vorgabe bzw. Handlung könnte sein, Kinder nur in den Kindergarten bzw. in die Schule gehen zu lassen, wenn sie z.B. die deutsche Sprache beherrschen. Diese restriktive Vorgabe einer Verwaltung könnte trotzdem zur einer erfolgreichen Integration beitragen, denn durch die Sprache ist eine kulturelle Annäherung gegeben, eine gesellschaftliche Plazierung wäre eher möglich, Interaktionen könnten schneller aufgebaut und eine Identifikation mit dem System könnte erfolgreich stattfinden (Esser, 2001). Eine zweite Handlungsmöglichkeit, Integration positiv zu beeinflussen, stellt die Solidarität mit Anderen und die Tolerierung der Anderen dar. Durch diese liberalen Handlungen, wie die Menschen, die sich durch Andersartigkeit auszeichnen, zu akzeptieren und sich solidarisch mit innen zu erklären, kann Integration positiv beeinflusst werden (Fuchs, 1999). Diese liberalen Handlungen haben die gleichen Auswirkungen auf die Integration bzw. deren Ausprägungen, wie oben beschrieben. Soll die Integration von Randgruppen durch Handlungen der Forstverwaltung erfolgen, ist davon auszugehen, dass sie dies, aufgrund ihres Aufgabenverständnisses, nicht ohne Instrumente bzw. Hilfestellung leisten können. Eine Kooperation mit Institutionen des sozialen Sektors könnte eine Strategie der Forstverwaltung darstellen, das Potential des Waldes für die soziale Nachhaltigkeit und soziale Integration zu erschließen. Eine zweite Möglichkeit der Forstverwaltung, die Integration von Problemgruppen zu fördern, kann in der Entwicklung eines neuen Produktes liegen, das auf dem Markt angeboten wird. Durch die Mitwirkung an der Förderung von Integration sozialer Problemgruppen kann sich für die Forstverwaltung in urbanen Zentren der eigene Handlungsspielraum erweitern. Weiterhin wird davon ausgegangen, dass durch die Strategie der Kooperation oder die Strategie der Produktentwicklung die Erschließung neuer Ressourcen für die Forstveraltung ermöglicht wird. 


\section{Theoretische Grundlagen}

Die theoretischen Grundlagen dieses Kapitels setzten sich aus der Definition von Randgruppen, der Theorie der sozialen Integration, der Kooperationstheorie, der Aufgabenwahrnehmung der Forstverwatung und der Produktentwicklung auf dem Markt zusammen.

\subsection{Der Randgruppenbegriff und soziale Ungleichheit}

Um Überlegungen zur Integration anzustellen, muss zunächst geklärt werden, auf welche Problem- bzw. Randgruppen sich die Integration beziehen soll und wie es zu sozialer Ungleichheit kommen kann.

Der Randgruppenbegriff lässt sich grob in drei Zyklen einteilen, in denen er auf verschiedene Art verwendet wurde. In den 50iger Jahren hatte der Randgruppenbegriff stigmatisierenden Charakter, indem man Personen als randständig bezeichnete, die aus persönlichem Versagen und eigener Schuld die Eingliederung in die Gesellschaft versäumt haben. Hilfestellung wurde zu dem Zeitpunkt in der Disziplinierung der Menschen gesehen. Zum Ende der 60iger und in den 70iger Jahren bezog sich der Begriff auf benachteiligte Bevölkerungsschichten, die als Unterprivilegierte galten und denen z.B. durch eigene Mobilisierung und Auflösen von sozialen Brennpunkten und später durch die generelle Forderung der Chancengleichheit geholfen werden sollte. Die Entwicklung in den 80iger Jahren rückt die Benachteiligungsprozesse in der Gesellschaft in den Mittelpunkt und umfasst somit den Kern und nicht nur den Rand der Gesellschaft. Die Entwicklung des Randgruppenbegriffs führte dazu, dass die Sozialarbeit und Sozialpädagogik heute weitgehend auf den Randgruppenbegriff verzichtet und die sozialen Faktoren, die realen Benachteiligungen und Lebenssituationen einzelner Gruppen erforscht und in den Vordergrund gestellt werden. So fand durch die Randgruppendiskussion eine Entwicklung zur mehrdimensionalen Betrachtung (ökonomisch und politisch) der Probleme statt und die Arbeit mit den Randgruppen hat die Aufhebung von Unterversorgung und Benachteiligung zum Ziel (Chassé, 1992). Mit dem Ergebnis der 2006 von der Friedrich - Ebert- Stiftung in Auftrag gegebenen Studie "Gesellschaft im Reformprozess" ist eine neue Debatte um die Unterschicht entbrannt. Die Studie zeigt eine Einteilung der Bevölkerung in neun politische Typen auf der Basis der politischen Wertvorstellungen und Einstellungen (Friedrich-EbertStiftung, 2006). Hier wird festgestellt, dass $8 \%$ der Wahlbevölkerung zum so genannten abgehängten Prekariat zu zählen sind. Diese Bevölkerungsgruppe ist u. a. gekennzeichnet durch eine einfache Bildung, einen hohen Arbeitslosenanteil, finanzieller Unsicherheit 
(niedriges Haushaltseinkommen, kaum finanzielle Rücklagen), einen hohen Männeranteil. Dieser Typ ist im Osten stark ausgeprägt und generell eher im ländlichen Raum zu finden (ebd.). So sehr der Randgruppenbegriff in der Soziologie in den Hintergrund getreten ist, umso erstaunter gestaltete sich das Echo auf die Ergebnisse der Studie, denn die Bemühungen der Sozialarbeit sollten eine abgehängte Bevölkerungsgruppe verhindern.

Bolte und Hradil (1988, S. 237) haben eine umfassende Definition des Randgruppenbegriffs geliefert:

„[...] daß bestimmte Gruppen der Bevölkerung [...] jeweils so gravierende Anhäufungen von sozialen Benachteiligungen auf sich vereinen, daß sie vom üblichen Leben in unserer Gesellschaft in gewisser Weise ausgeschlossen sind und daher „randständig“ erscheinen. Randständigkeit kann sich sowohl aus ungünstigen materiellen Lebensbedingungen (z.B. Armut oder Wohnungslosigkeit) als auch aus einem besonderen Verhältnis zu staatlichen Stellen (Betreuung, Aufsicht, Haft etc.) oder aus problematischen Beziehungen zur übrigen Bevölkerung (Vorurteile, Diskriminierung, Isolation o. ä.) ergeben."

Von dieser Randständigkeit sind Behinderte, Obdachlose und Nichtsesshafte, ausländische Arbeiter, Drogenabhängige, Strafentlassene, aus stationärer Therapie Entlassene sowie langjährige Insassen von Strafanstalten, psychiatrischen Krankenhäusern, Pflege- und Fürsorgeheimen betroffen (Bolte u. Hradil, 1988, S. 238). Zu den Randgruppen sind ebenfalls Langzeitarbeitslose und problematische Jugendliche zu zählen, die in der vorliegenden Arbeit Berücksichtigung finden. Die Randgruppen der o. g. Auflistung unterscheiden sich untereinander durch die Art der Benachteiligung und die spezifischen Lebenslagen. Innerhalb einer Gruppe herrschen jedoch charakteristische Gemeinsamkeiten vor, die deren soziale Lage, Mentalität und Verhaltensweisen betreffen. Bei einigen Personengruppen handelt es sich um umfangreiche Bevölkerungsgruppen, die nicht mehr als „Randerscheinung“ zu bezeichnen sind (ebd.). So waren in Deutschland im Dezember 2003 6,6 Mill. Menschen als Schwerbehinderte anerkannt, was einem Bevölkerungsanteil von ca. 8,0\% entspricht (Statistisches Bundesamt, 2005). Der Randgruppenbegriff ist eng mit der Diskussion um die Soziale Ungleichheit verbunden. Bolte und Hradil (1988) stellen heraus, dass in der Gesellschaft sozial relevante Differenzierungen vorherrschen, die das Zusammenleben beeinflussen. So sind generelle Unterschiede zwischen den Menschen in Bezug auf das Geschlecht, das Alter, den Beruf, den Familienstand, die Zugehörigkeit zu Vereinen oder Konfessionsgemeinschaften etc., für das Leben und Zusammenleben von Menschen von großer Bedeutung (ebd., S. 22). Werden den sozialen Differenzierungen, die Menschen als verschieden oder übereinstimmend charakterisieren (z.B. Bildungsstand), ,Bewertungen' zugeschrieben, handelt es sich um Erscheinungen der sozialen Ungleichheit. Diese Bewertungen differenzieren die Menschen im Sinne von gleich-, besser-, schlechter, höher- oder tiefergestellt (ebd., S. 23). Die soziale Ungleichheit schlägt sich in verschiedenen Ausprägungen nieder, wie z.B. die berufliche Über- und Unterordnung, die Abstufung zwischen ärmeren und reicheren Menschen, die Zugehörigkeit zu mehr oder weniger 
angesehenen Gruppierungen oder besser bzw. schlechteren Wohn-, Arbeits- und Freizeitbedingungen (ebd.). $\mathrm{Zu}$ den Bewertungen und Ausprägungen von sozialer Ungleichheit werden auch die Merkmale hinzugezogen, die von den Menschen beeinflussbar und nicht beeinflussbar sind. Bolte und Hradil (1988) beschreiben diese Merkmale als ,ascribed positions', wie Geschlecht und Herkunft, die einem Menschen unbeeinflussbar zugeschrieben sind und als ,achieved positions', wie der Beruf, eben die Merkmale, die von Menschen erreicht oder erworben werden können (ebd., S. 23). So können soziale Ungleichheiten zusammenfassend als Merkmale bezeichnet werden, die Unterschiede der Lebenssituation von Menschen als gleich-, besser- oder schlechter, höher- oder tiefergestellt bewerten bzw. ungleiche Lebenslagen aufzeigen (ebd.). Die oben erwähnten Randgruppen befinden sich in Lebenslagen, die als schlechter einzustufen sind, weil sie von einer Vielzahl von Benachteiligungen betroffen sind, die sie in der Beurteilung an den Rand der Gesellschaft stellen.

Kreckel (2005) empfiehlt eine begriffliche Präzisierung des sozialen Ungleichheitsbegriffs. Soziale Ungleichheit sei zunächst von der physischen Verschiedenartigkeit der Menschen, z.B. Geschlecht, Alter, Hautfarbe etc., zu unterscheiden und beziehe sich auf die „Begünstigung“ oder „Bevorrechtigung“ bzw. „Benachteiligung“ und Diskriminierung von Menschen in einer Gesellschaft (ebd., S. 1730). Weiterhin differenziert Kreckel (2005) soziale Ungleichheit und soziale Differenzierung. Die soziale Differenzierung spiegelt die sozial verankerten Unterschiedlichkeiten der Menschen wider, wie z.B. berufliche Arbeitsteilung, Religionszugehörigkeit, regionalen oder nationalen Besonderheiten. Diese Unterschiede müssen nicht zwangsläufig zu einer Benachteiligung oder Bevorzugung in der Gesellschaft führen. So können Menschen mit unterschiedlicher Sprache und Hautfarbe gleichberechtigt in einer Gesellschaft zusammenleben. Es kann aber auch gerade aus diesen Gründen zu Diskriminierungen und Benachteiligungen kommen. Ein Beispiel hierfür stellt die berufliche Differenzierung in der Gesellschaft dar. Es existieren differenzierte Berufe, die aber gleichgestellt und gleichgeachtet nebeneinander betrachtet werden, z.B. verschiedene Handwerksberufe. In anderen Berufen dominieren die hierarchischen Beziehungen, z.B. Arzt und Krankenschwester, Ingenieur und Facharbeiter. Ein Ideal von sozialer Gleichheit wäre hier die gleichwertige Annerkennung von Denk- und Verhaltensmöglichkeiten der Mitglieder einer Gesellschaft, die daraufhin nicht zu einseitigen Benachteiligungen oder Begünstigungen führen (ebd., S. 1731). Soziale Ungleichheit liegt nach Kreckel (2005) vor, wenn soziale Differenzierungen bestehen, die Individuen oder Gruppen einer Gesellschaft dauerhaft begünstigen und andere benachteiligen (ebd.). Er nimmt folgende Präzisierung des Begriffes vor:

„Soziale Ungleichheit liegt dort vor, wo die Möglichkeiten des Zugangs zu allgemein verfügbaren und erstrebenswerten sozialen Gütern und / oder zu sozialen Positionen, die mit ungleichern Macht- und / oder Interaktionsmöglichkeiten ausgestattet sind, dauerhafte 
Einschränkungen erfahren und dadurch die Lebenschancen der betroffenen Individuen, Gruppen oder Gesellschaften beeinträchtigt oder begünstigt werden. (ebd., S. 1731)

Wird der Begriff der sozialen Ungleichheit diskutiert, sollte auch das ,abweichende Verhalten' von Gesellschaftsmitgliedern betrachtet werden. Dieses abweichende Verhalten wird von großen Teilen der Gesellschaft als „ungewöhnlich, befremdlich und jenseits des durchschnittlichen Erwartungshorizonts wahrgenommen“ (Keupp, 1976, S. 13). Menschen bzw. Gruppen der Gesellschaft, die abweichendes Verhalten zeigen, verletzen auf verschiedene Art den Verhaltenskonsens, der in einer Gesellschaft bzw. deren Gruppen als Regel verankert ist (ebd.). So werden Gruppen bzw. Individuen, die von der Verhaltensnorm der Gesellschaft abrücken zu Außenseitern, Ausgeschlossenen oder Randgruppen. Keupp (1976) setzt sich hierzu kritisch mit dem Labeling-Ansatz auseinander, dessen Hauptvertreter Lemert ist. Dieser besagt, dass unterschiedliche Ursachen für ein primäres, abweichendes Verhalten von Individuen oder Gruppen verantwortlich sind, wie z.B. soziale oder kulturelle Faktoren, die sich aber nur am Rande auf den Status und die psychologische Struktur der Personen auswirken (Keupp, 1976, S. 32). Wird diesem primären, abweichenden Verhalten von Individuen, Gruppen oder Institutionen Bedeutung und Aufmerksamkeit geschenkt und werden aufgrund der Bedeutungen Handlungen ausgelöst, entsteht ein sekundäres, abweichendes Verhalten (ebd.). Die betreffenden Personen werden zunächst mit dem „Etikett“ (Label) abweichend versehen, weil es sich nicht um ein alltägliches, routinehaftes Verhalten handelt und dies führt zu gesellschaftlichen Reaktionen. Hieraus entsteht das sekundäre, abweichende Verhalten der betroffenen Personen, welches sich gravierender auf den Status und die psychologische Struktur auswirkt, als das primäre, abweichende Verhalten. Die Individuen werden so auf den abweichenden Status festgelegt und in diesem auch verstärkt (ebd., S. 35). Kitsuse fasst den Labeling-Ansatz folgendermaßen zusammen: Das Verhalten von Menschen in einer Gesellschaft führt nicht zu einer Unterscheidung zwischen abweichenden und nicht-abweichenden Personen, sondern erst die Reaktionen und Definitionen der ,normalen', angepassten Mitglieder der Gesellschaft verwandeln durch die Interpretation und Identifikation von abweichendem Verhalten, Menschen zu ,Abweichlern' (nach Keupp, 1976, S. 33).

Nach ausführlicher Darstellung des Randgruppenbegriffs und der Entstehung von sozialer Ungleichheit soll aufgezeigt werden, wie die Integration und der Abbau von sozialer Ungleichheit theoretisch vollzogen werden kann. 


\subsection{Soziale Integration}

Allgemein bezieht sich Integration auf die Differenz vom Ganzen und Teilen, wobei die Integration die Verbindung von den Teilen zu einem Ganzen darstellt (Fuchs, 1999, S. 150). Die Teile des Ganzen müssen demnach in Verbindung zueinander stehen, d.h. in einer Gesellschaft müssen die Individuen durch Interaktionen miteinander verbunden sein. Interaktionen zwischen Mitgliedern einer Gemeinschaft kommen aber nur dann zustande, wenn diese koordiniert, aufeinander bezogen und wechselseitig anschlussfähig sind (ebd., S. 151). Die Handlungen von Akteuren in einer Gemeinschaft sind an generalisierte Verhaltenserwartungen gebunden, die festlegen, welche Handlungen in Situationen typischerweise und normativ erwartet werden können (ebd.). Dies bedeutet, dass in einer Gesellschaft Handlungsnormen bestehen, die bei Nichtbeachtung der Mitglieder mit Sanktionen bedacht werden. Die Handlungsnormen können in zwei generelle Bereiche aufgeteilt werden. Zum einen bestehen soziale Normen, die ein Akteur befolgen sollte und zum anderen soll er sich an rechtliche Normen halten. Die sozialen und staatlichen Sanktionen, die bei Nichtbeachtung bzw. Nichtbefolgung der Normen eintreten, sind von unterschiedlichem Ausmaß. Die Stärke der staatlichen Sanktionen ist gegenüber der Stärke der sozialen Sanktionen als höher einzustufen (ebd., S. 152f.). So kann die staatliche Sanktion für die Regelverletzung z.B. einen Freiheitsentzug bedeuten, wohingegen sich die soziale Sanktion für einen Betrug in dem Ausschluss aus einer Gemeinschaft (z.B. Freundeskreis, Verein) ausdrückt. Sanktionen und Handlungen der Akteure sind an Handlungsregeln gebunden. Diese Handlungsregeln für die Akteure müssen zentral zusammengefasst und überprüfbar sein. Diese Aufgabe kann von politischen Institutionen übernommen werden, die zum einen selbst als Regel-Komplexe gesehen werden können und zum anderen die Funktion besitzen, Entscheidungen herzustellen und durchzusetzen, die für eine gesellschaftliche Gemeinschaft bindend sind (ebd., S. 164). Der Zusammenhang von politischen Institutionen (soziologische Definition) und der sozialen Integration soll Grundlage des folgenden Kapitels sein. 


\subsubsection{Liberales Modell der Sozialen Integration}

Soziale Integration setzt zunächst die Annahme eines demokratischen System voraus, welches aus den drei Ebenen Kultur, Struktur und Prozess besteht.

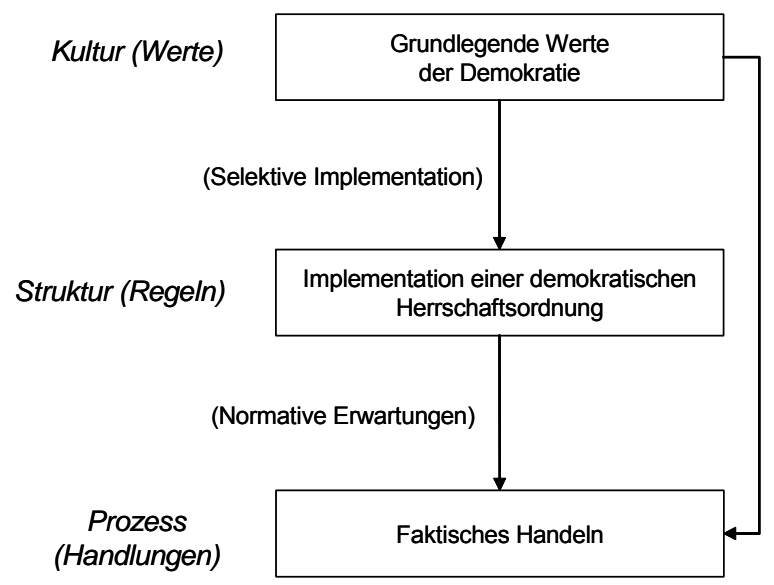

Abbildung 1: Ebenen der Demokratie (vgl. Fuchs, 1999, S. 165)

Diese Ebenen sind hierarchisch nach den Elementen, die sie festsetzen, angeordnet. Die höchste Hierarchieebene ist die Kulturebene, auf der die Werte verankerte sind. Es handelt sich um die grundlegenden Werte der Demokratie, wie z.B. die Annerkennung aller Menschen als frei und gleich. Durch die Festlegung bestimmter demokratischer Werte findet eine selektive Implementation statt, die sich auf der Strukturebene in den Regeln einer demokratischen Herrschaftsordnung manifestiert. Durch die Aufstellung von Regeln werden an die Prozessebene normative Erwartungen gestellt, die sich in faktischen Handlungen widerspiegeln.

„Die soziale Integration einer gesellschaftlichen Gemeinschaft bezieht sich auf die Koordination der Handlungen der Mitglieder dieser Gemeinschaft. Die Koordination erfolgt durch Regel-Strukturen, die aus generalisierten Verhaltenserwartungen der Akteure in konkreten Handlungssituationen bestehen, welche sich in bestimmten Handlungskontexten ergeben. Die Handlungskoordination - und damit auch die soziale Integration - ist in dem Maße erfolgreich, in dem die Akteure den generalisierten Verhaltenserwartungen tatsächlich entsprechen." (Fuchs, 1999, S. 165f.)

Bei der Koordination von Handlungen, ergeben sich zwangsläufig auch Konflikte. Zum einen können diese als interpersonelle Konflikte bezeichnet werden und zum anderen als Konflikte, die sich aus der Festlegung und Verwirklichung kollektiver Ziele ergeben (ebd., S. 166). Fuchs (1999) stellt an dieser Stelle fest, dass sich Integration einer Gemeinschaft nur ergeben kann, wenn Handlungsregeln bestehen, die einen allgemeinen Geltungsanspruch erheben und auch im Zweifel kontrafaktisch durchgesetzt werden. Die Grundlage von Integration können somit Rechts-Normen darstellen, die für alle Mitglieder einer Gemeinschaft bindend sind und auch bei abweichendem Verhalten für alle gleich 
durchgesetzt werden. Um soziale Integration zu untersuchen, wird als Grundlage der Handlungsregeln die Bindung der Akteure an die Verfassung gesehen (s. Abb. 1).

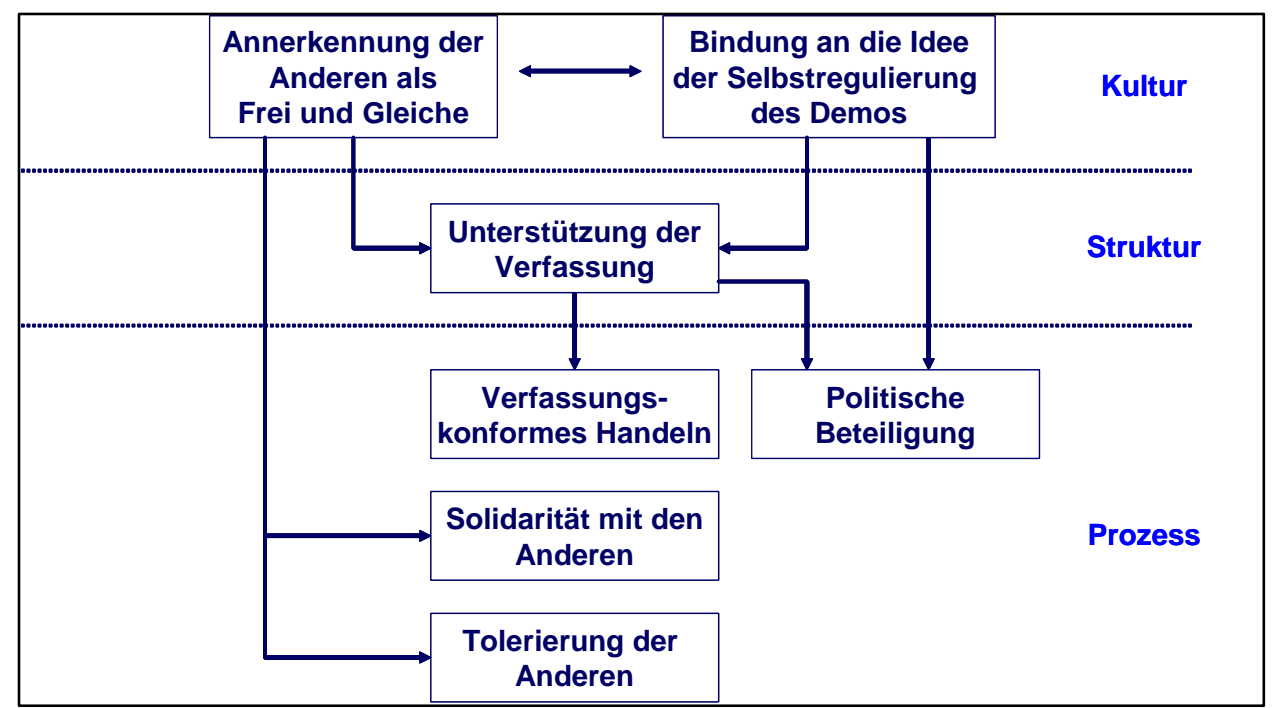

Abbildung 2: Liberales Modell der sozialen Integration (nach Fuchs, 1999, S. 169)

Auf der Kulturebene des „Liberalen Modells der sozialen Integration“ (Fuchs, 1999), auf dem das Forschungsvorhaben basiert, sind die grundsätzlichen liberalen Werte nach der liberalen Demokratietheorie zu finden, wie die Annerkennung der Anderen als Freie und Gleiche und der Demokratiegrundsatz (Selbstregulierung des Demos, Letztbestimmungsrecht des Volkes). An dieser Stelle wird also die Zustimmung der Akteure zu den Grundwerten, die sich aus der Verfassung ergeben, erfragt. Diese Zustimmung auf der Kulturebene soll sich in aktives Engagement zur Unterstützung der Verfassung auf der Strukturebene entwickeln. Die aktive Unterstützung veranlasst die Bürger zu einem Verfassungskonformen Handeln und zu Politischer Beteiligung, mit der minimalen Anforderung Beteiligung an Wahlen. Diese Faktoren sind der dritten Ebene des Modells, der Prozessebene zuzuordnen.

\begin{tabular}{|c|c|c|c|}
\hline $\begin{array}{l}\text { 1. Kategorie politischen } \\
\text { Handelns }\end{array}$ & $\begin{array}{c}\text { Verfassungs- } \\
\text { konformes Handeln }\end{array}$ & $\begin{array}{l}\text { Politische } \\
\text { Beteiligung }\end{array}$ & $\begin{array}{l}\text { Beteiligung } \\
\text { an Wahlen }\end{array}$ \\
\hline \multirow{2}{*}{\multicolumn{4}{|c|}{$\underset{\substack{\text { Solidarität mit den } \\
\text { Anderen }} \rightarrow \begin{array}{l}\text { Handlungsebene }=\text { Aktivitäten der } \\
\text { Bürger }\end{array}}{ }$}} \\
\hline & & & \\
\hline $\begin{array}{l}\text { 2. Kategorie politischen } \\
\text { Handelns }\end{array}$ & $\begin{array}{l}\text { Tolerierung der } \\
\text { Anderen }\end{array}$ & \multicolumn{2}{|c|}{$\begin{array}{l}\text { Respekt, Toleranz = Akzeptanz der } \\
\text { Andersartigkeit }\end{array}$} \\
\hline
\end{tabular}

Abbildung 3: Prozessebene des Liberalen Modells der sozialen Integration

Die Prozessebene kann in zwei Kategorien politischen Handelns eingeteilt werden, wobei das Verfassungskonforme Handeln und die Wahlbeteiligung die beiden Faktoren der ersten 
Kategorie widerspiegeln. Nach Auffassung der neoliberalen Demokratietheorien wäre mit der Erfüllung dieser beiden Faktoren bzw. der ersten Kategorie des Handelns ein ausreichendes Maß an Integration gegeben. Diese Auffassung ist nach der liberalen Demokratietheorie normativ zu anspruchslos. So gilt eine gesellschaftliche Gemeinschaft erst als integriert, wenn auch die zweite Kategorie politischen Handelns erfüllt ist. Die zweite Kategorie politischen Handelns auf der Prozessebene ist gekennzeichnet durch die Faktoren Solidarität mit Anderen und Tolerierung der Anderen. Besonders wichtig ist hierbei, dass die Konstrukte bzw. Faktoren als Handlungen verstanden werden, was sich in Aktivitäten der Bürger ausdrückt. Solidarität wird zunächst als gesellschaftstheoretischer Begriff verstanden und meint die wechselseitige Verantwortung und wechselseitiges Einstehen der Mitglieder einer gesellschaftlichen Gemeinschaft. Solidarität auf der Handlungsebene (Prozessebene) wird durch die Vermeidung von Diskriminierung und Marginalisierung sozialer Gruppen der gesellschaftlichen Gemeinschaft als aktive Handlung festgemacht (Fuchs, 1999, S. 158). Die Solidarität mit Anderen als Aktivität der Bürger kann in zwei Formen ausgedrückt werden. Zum einen können sich Akteure an nicht-staatlichen Organisation und Initiativen beteiligen, welche die Zielsetzung Vermeidung von Diskriminierung und Marginalisierung vertreten. Zum anderen können Akteure ihren Anspruch an politische Eliten geltend machen, der zu einem Kriterium der Wahlentscheidung wird und so eine Handlungsrelevanz erhält (ebd., S. 169). Der zweite Aspekt der zweiten Kategorie politischen Handelns ist als Tolerierung der Anderen zu bezeichnen. Die Aktivitäten der Bürger sollen hier den wechselseitigen Respekt oder die wechselseitige Toleranz ausdrücken. Dies soll aber nicht auf der abstrakten Ebene geschehen, auf der Alle als frei und gleich angesehen werden, sondern soll sich in der Akzeptanz und Wahrnehmung der Andersartigkeit (Identität) und Besonderheit manifestieren (ebd.). Die Annerkennung der Anderen in ihrer Andersartigkeit ist schon auf der Kulturebene zu finden und soll nun in Handlungen auf der Prozessebene ausgedrückt werden. Um die Tolerierung der Anderen als Handlung zu begreifen, müssen sprachliche und gestische Äußerungen als Ausdruck der Tolerierung angesehen werden. Dieser Ausdruck von eigenen Wertung, verstanden als Handlungen, kann in Befragungen durch missbilligende oder respektvollen Äußerungen ermittelt werden (ebd., S. 170). Die Dimension Tolerierung der Andern als Faktor des „Liberalen Modells der sozialen Integration“ lässt eine normativ anspruchsvolle Definition von sozialer Integration zu:

\footnotetext{
„Je stärker die Tolerierung der Anderen in den Interaktionen der Mitglieder einer gesellschaftlichen Gemeinschaft, desto größer ist die soziale Integration dieser Gemeinschaft (und vice versa). (Fuchs, 1999, S. 170)
}

Die Definition von sozialer Integration soll in der Untersuchung durch die Befragung von Experten überprüft werden. Dies soll durch die Befragung von Akteuren zur Integration von Problem- bzw. Randgruppen geschehen, wobei auf deren sprachlichen und gestischen Äußerungen abgezielt wird. In der Untersuchung liegt das Hauptinteresse auf den Aktivitäten 
(Äußerungen) der Forstverwaltungen in urbanen Zentren und denen der an Projekten beteiligten Organisationen.

\subsubsection{Formen der Sozialintegration}

Lockwood (1979, S. 125) unterscheidet in der Diskussion des sozialen Wandels der Gesellschaft zwischen Sozialer Integration und Systemintegration. Bei der Sozialen Integration geht es um die Beziehungen (geordnet oder konflikthaft) der Handelnden eines sozialen Systems. Die Systemintegration hingegen beschäftigt sich mit den Beziehungen (geordnet oder konflikthaft) zwischen den Teilen eines sozialen Systems. Sozialer Wandel der Gesellschaft soll hier als Umbildung der herrschenden institutionellen Ordnung einer Gesellschaft verstanden werden (ebd., S. 124).

Esser (2000, S. 261f.) beschreibt Integration als:

„Unter Integration wird generell der Zusammenhalt von Teilen in einem „systemischen“ Ganzen und die dadurch erzeugte Abgrenzung von einer unstrukturierten Umgebung verstanden, gleichgültig zunächst worauf dieser Zusammenhalt beruht. Die Teile müssen, wie man auch sagen könnte, „,integraler“, also ein nicht wegzudenkender, Bestandteil des Ganzen sein."

Im Kontext der Integration von Menschen mit Migrationshintergrund ist mit Sozialintegration die Einbeziehung der Akteure in das gesellschaftliche Geschehen, wie z.B. die Gewährung von Rechten, der Erwerb von Sprachkenntnissen, die Beteiligung am Bildungssystem und dem Arbeitsmarkt etc. Weiterhin werden vier Varianten der Sozialintegration aufgeführt, die auch auf andere zu integrierende Personengruppen bezogen werden können (Esser, 2001).

1. Kulturation. Diese Variante der Sozialintegration setzt voraus, dass die Akteure Wissen und Kompetenzen besitzen, um sinnhaft, verständig und erfolgreich Agieren und Interagieren zu können. Hierbei stehen das Wissen und die Kompetenz der wichtigsten Regeln und die Beherrschung der kulturellen Fertigkeiten im Vordergrund. Diese Fertigkeiten beziehen sich vornehmlich auf die Sprache. Um am gesellschaftlichen Leben teilzunehmen, wie z.B. an interessanten Interaktionen oder an der Besetzung von angesehenen Positionen, muss in Wissen, Kompetenz und sprachliche Fertigkeit investiert werden. Die Sozialintegration als Kulturation kann als Prozess verstanden werden, in welchem Wissen und Kompetenzen erworben werden. Gerade die sprachliche Fertigkeit ist bei Ausländern und Menschen mit Migrationshintergrund ein entscheidender Faktor zur sozialen Integration. Aber auch der Erwerb von diversen Kompetenzen, wie z.B. eine Arbeitsmethode oder Kompetenz über das öffentliche Hilfesystem, kann Arbeitslosen oder Hilfebedürftigen den Zugang in die Gesellschaft erleichtern bzw. verschaffen. 
2. Plazierung. Hierunter wird allgemein die Besetzung einer gesellschaftlichen Position durch einen Akteur verstanden. Durch die Plazierung wird ein Akteur in ein schon bestehendes soziales System aufgenommen und eingegliedert. Die soziale Integration durch Plazierung findet durch die Verleihung bestimmter Rechte statt, wie z.B. das Wahlrecht, die Übernahme beruflicher und anderer Positionen und die Möglichkeit, soziale Gelegenheiten wahrzunehmen, um soziale Beziehungen zu anderen Mitgliedern des sozialen Systems zu aufzubauen oder zu unterhalten. Esser (2001, S. 9) spricht im Hinblick auf die Plazierung von einer „Spezialvariante des Marktgeschehens“. Die Akteure tätigen „Angebote“ in der Form von Fertigkeiten und Ressourcen z.B. auf dem Arbeitsmarkt, dem Wohnungsmarkt oder auch dem Beziehungsmarkt, wobei andere Akteure diese Angebote nachfragen. Die erfolgreiche Plazierung eines Akteurs hängt aber auch von „Entscheidungen“ anderer Akteure ab, so. z.B. Lehrer, die eine weiterführende Schule empfehlen oder Arbeitgeber die über die Einstellung entscheiden. Diese Entscheidungen sind von instrumentellen (z.B. hohe Produktivität eines Bewerbers) und nicht-instrumentellen Beweggründen (z.B. Sympathie oder Antipathie) der Entscheidenden abhängig. Somit können nicht-instrumentelle Beweggründe bzw. „Vorurteile“ und Diskriminierung eine erfolgreiche Plazierung von Akteuren behindern und erschweren. Zwischen der Kulturation und der Plazierung als Formen der Sozialintegration bestehen Zusammenhänge. Zum einen erwerben die Akteure durch die Plazierung in der Gesellschaft bestimmte Kompetenzen und Wissen. Zum anderen ist die Kulturation ein wichtiger Faktor für die Plazierung eines Akteurs, wie z.B. wer eine gute Schulausbildung genossen hat, kann eine gute berufliche Position erlangen. Durch die Erlangung von Kompetenzen hat ein Akteur Zugang zu interessanten Ressourcen, die inn als Person oder in einer Gruppe wiederum interessant für andere Akteure des Systems macht. Der Zugriff auf Ressourcen, wie ökonomisches Kapital, Humankapital (Wissen, Kompetenz etc), institutionelles Kapital (Rechte) und politisches Kapital (Vertretung der eigenen Interessen) verleiht dem Akteur allgemein Macht, sichert die Plazierung des Akteurs und so eine nachhaltige Sozialintegration.

3. Interaktion: Akteure treten in diesem speziellen Fall von sozialem Handeln über Wissen und Symbole in Relationen und orientieren sich aneinander. Es existieren drei Spezialfälle der Interaktion, nämlich die gedankliche Koorientierung, die symbolische Interaktion und die Kommunikation, die hier nur kurz Erwähnung finden sollen. Interaktionen können als soziale Beziehungen mit mehr oder weniger festen und verbindlichen Regeln für typische Arten der Interaktionen bezeichnet werden. So tragen Beziehungen, wie eine Nachbarschaft, eine Freundschaft oder ein eheliche 
Beziehung zur sozialen Integration bei. Ein Kennzeichen von Integration durch Interaktionen ist der emotionale Bezug. Demnach können „Dissonanzen“ hemmend auf die Aufnahme von Interaktionen wirken, die es bei wechselseitigem Interesse an der Interaktion abzubauen gilt.

4. Identifikation: identifiziert sich ein Akteur mit dem sozialen System, so sieht er sich und das soziale Gebilde als Einheit an, ist mit inm „identisch“. Die Identifikation ist als gedankliche und emotionale Beziehung zwischen dem einzelnen Akteur und dem sozialen System (Ganzheit, Kollektiv) zu verstehen, wobei sich der Akteur z.B. an dem Nationalstolz oder dem Wir-Gefühl zu anderen Mitgliedern der Gesellschaft oder Gruppe orientiert. Die soziale Integration durch Identifikation kann in drei unterschiedlich intensiven Formen stattfinden.

A) Werte: Die Wertintegration stellt die Identifikation der Akteure mit dem sozialen System als Kollektiv dar, z.B. einer Gesellschaft, einer Gruppe oder einer Organisation. Die emotionale Unterstützung der Institutionen, die Zurücknahme der individuellen und egoistischen Motive, das ausgeprägte Gefühl der Solidarität mit dem System und die unbedingten Werte sind Kennzeichen dieser Form der sozialen Integration.

B) Bürgersinn: Als Form der Unterstützung der Gesellschaft geht es vornehmlich um die Sicherung der Verfassung, die ein Eindringen kollektiver und „fundamentalistischer" Ansprüche abwehren soll.

C) Hinnahme: Auch die Hinnahme spiegelt eine Form der Identifikation und Unterstützung der Gesellschaft wider und ermöglicht soziale Integration. Zum einen nehmen die Akteure das System hin, weil eine Vielfalt von internen Konflikten und Orientierungen vorherrscht und die Akteure nicht mehr zu einer Gruppe mit gleichen Interessen zusammengeschlossen werden kann. Durch diese „Individualisierung“ lehnen sich die Akteure nicht gegen das System auf und nehmen dies hin (Verkettungsintegration). Auch die Aussichtlosigkeit das System in irgendeiner Form zu verändern, lässt einige Akteure das System desillusioniert hinnehmen, wobei hier von Deferenzintegration gesprochen wird und eine der schwächsten Form der sozialen Integration darstellt.

Zusammenfassend kann festgestellt werden, dass die unterschiedlichen Formen der Sozialintegration Schnittstellen aufweisen und nicht trennscharf voneinander zu betrachten sind, wie bei der Ausführung von Kulturation und Plazierung schon erwähnt wurde. Um nachhaltig eine Sozialintegration zu gewährleisten, sollten sich Akteure auf einer zentralen und für alle Akteure interessanten Position plazieren. Die Position und die Kontrolle von Kompetenzen sind wiederum wichtige Bedingungen der Systemintegration und deren Mechanismen Markt, Organisation und Medien, die so beeinflußt werden können. So kann 
die Positionierung und somit die Machtstellung als Voraussetzung für alle Prozesse der Integration angesehen werden (vgl. Esser, 2001, S. 8-14). Die Formen der Sozialintegration lassen sich auf alle Personen und Personengruppen innerhalb einer Gesellschaft beziehen.

\subsubsection{Exkurs: Potential des Waldes für die soziale Integration}

In einem Exkurs soll das Potential des Waldes für die soziale Integration anhand der theoretischen Ausführungen abgeleitet und präzisiert werden. Die Formen der Sozialintegration stehen hierbei im Vordergrund. Die vier Sozialintegrationsformen von Esser (2001) werfen die Frage auf, welchen Beitrag der Wald zu diesen einzelnen Stufen leisten und wie dieser konkretisiert werden kann.

Die Kulturation (Erwerb von Wissen, Kompetenzen, sprachlichen Fertigkeiten), als Prozess, soll das Interagieren und so die Sozialintegration der betroffenen Personengruppen fördern. Die Möglichkeiten, die der Wald für die Kulturation bieten könnte, wären im Bereich der Wissensvermittlung denkbar. Der Wald kann als Lern- und Lehrraum für Menschen fungieren, die eine sprachliche Barriere zu überwinden haben. Vorstellbar wäre das Angebot von Sprachkursen bzw. Kurseinheiten im Wald. Da der Wald ein positiv besetztes Element in fast allen Kulturkreisen ist, kann er somit ein Medium darstellen, in dem Sprache lebendig erlernt bzw. gelehrt werden kann. Ein weiterer Aspekt soziale Integration über Kulturation zu fördern, stellt der unterschiedliche Zugang zum Wald dar. Die verschiedenen Kulturen haben ein unterschiedliches Verständnis von der Nutzung und dem Umgang mit dem Wald. Um Integration zu schaffen, könnten diese Unterschiede im Wald aufgezeigt und erfahrbar gemacht werden, z.B. durch die Zuweisung von Waldarealen, die nach den kulturellen Hintergründen bearbeitet werden könnten. Durch die Auseinandersetzung mit den verschiedenen Kulturen werden Wissen und Kompetenzen erworben, die Integration und Toleranz insgesamt positiv beeinflussen.

Die zweite Form der Sozialintegration, die Plazierung, bezeichnet die Aufnahme von Personen in ein schon bestehendes soziales System. Die Plazierung innerhalb der Gesellschaft hängt eng mit der Position auf dem Arbeitsmarkt zusammen. Wald könnte hierzu ebenfalls einen Beitrag leisten. Durch die Beschäftigung von Arbeitslosen im Wald könnten die Personen Kenntnisse und Fähigkeiten erwerben, die sie in die Lage versetzen, auf dem Arbeitsmarkt Fuß zu fassen. Eine direkte Integration durch einen Arbeitsplatz im Forstbetrieb wäre ein optimaler Beitrag, der aber aufgrund von Personalabbau kaum zu leisten ist. Es stellt sich hier die Frage, ob eine Daueranstellung im Wald von Arbeitslosen überhaupt angestrebt wird, da es sich um körperlich harte Arbeit handelt (ein Großteil der Langzeitarbeitslosen wäre dazu körperlich nicht mehr in der Lage). Die Position und die 
Eingliederung von Personen in den Arbeitsmarkt kann die Forstverwaltung über Beschäftigungsmaßnahmen dennoch beeinflussen. Durch die Arbeit im Wald können wichtige Fähigkeiten vermittelt werden, die ganz allgemein im Berufsleben von Bedeutung sind. So kann der Umgang mit Maschinen, die Arbeit im Team, die soziale Kompetenz, die Verantwortung für andere, die Planung von Arbeitseinsätzen, der geregelte Tagesablauf etc. erlernt werden. Diese Faktoren sind nicht nur für die Arbeit im Wald wichtig, sondern können auf alle Berufe übertragen werden. Kann die Forstverwaltung zum Erwerb dieser Fähigkeiten beitragen, die zu einer besseren Plazierung der Personengruppen führen, ist ein ganz entscheidender Anteil für die Sozialintegration geleistet.

Die Interaktion, als Form der Sozialintegration, nimmt Bezug auf die emotionalen Beziehungen der Personen zu ihrem Umfeld. Den Beitrag den der Wald und die Forstverwaltung zu diesem Aspekt leisten können, liegt in der Bereitstellung des Waldes für Begegnungen. Wird Wald als Raum genutzt, wo sich Menschen begegnen und austauschen, einander kennenlernen und Beziehungen aufbauen können, ist der Grundstein für die Sozialintegration durch Interaktion gegeben. Im Wald können Treffpunkte gestaltet und eingerichtet werden, die von allen gesellschaftlichen Gruppen genutzt werden und so können bei wechselseitigen Interessen Interaktion zwischen unterschiedlichen gesellschaftlichen Gruppen aufgebaut werden. Die Gestaltung von Veranstaltungen im Wald, wie Feste, Theater, Konzerte etc., die von einer bestimmten Gruppe organisiert werden, würden die Interaktionen der Menschen fördern. Den Wald als Interaktionsraum zur Verfügung zu stellen, ist zudem eine kostengünstige Alternative zu einem Begegnungszentrum in einem festen Gebäude.

Die vierte Form der Sozialintegration, die Identifikation, kann auch von Seiten der Forstverwaltung bedient werden. Hierbei geht es generell um die gedankliche und emotionale Bindung zwischen der einzelnen Person und dem gesamten sozialen System. Ein Zugehörigkeitsgefühl kann durch die Übernahme von Verantwortung entstehen. Durch den Einsatz im Bereich des Waldschutzes, z.B. Kontrolle von Käferbäumen, Käferfallen oder das Errichten eines Zaunes für eine Anpflanzung, wird einer Person Verantwortung für die Vitalität des Waldes übertragen. Mit der Übernahme von Verantwortung entsteht ein Gefühl der Bindung an den Wald, was mit anderen Menschen geteilt werden kann. Dies führt zu einer Identifikation der Personen mit Anderen, dem Wald und somit dem gesamten System, da Leistungen erbracht werden, die gerade diesem System nutzen.

Abschließend ist festzuhalten, dass die Forstverwaltung zu den Formen der Sozialintegration von Personengruppen einen Beitrag leisten kann. Der Beitrag liegt nicht immer offen auf der Hand, so dass ein Umdenken im Forstbetrieb stattfinden muss und bei der Umsetzung der Maßnahmen Partner hinzugezogen werden müssen. 


\subsection{Kooperative Verwaltung}

Das kooperative Verhalten findet sich in der alltäglichen Arbeitsroutine der öffentlichen Verwaltungen wieder. Dies lässt sich darin feststellen, dass Entscheidungen durch Verhandlungen mit Betroffenen vorbereitet werden, Eingriffe erst vorgenommen werden, wenn eine einvernehmliche Problemlösung nicht erreicht werden kann und anstelle von einseitigen Anordnungen, Absprachen und Verträge eingesetzt werden (Benz, 1994, S. 13). Kooperationsbeziehungen sind in allen Politikbereichen zu finden, so auch als prägendes Merkmal der Sozialpolitik. Hierbei ist die Zusammenarbeit zwischen öffentlichen Aufgabenträgern und privaten oder freigemeinnützigen Organisationen vor allem an den Stellen erforderlich, an denen selbstständige Einrichtungen die Leistung erbringen müssen. Ein Beispiel stellt hier die Kooperation zwischen staatlichen, kommunalen und nichtstaatlichen Organisationen in der Umsetzung von Sozialleistungsprogrammen dar, z.B. Arbeitsförderungsmaßnahmen und die Eingliederung von Menschen mit Behinderungen in den Arbeitsprozess (ebd., S. 25). Kooperatives Verwaltungshandeln zeichnet sich vor allem darin aus, dass sich Beziehungen zwischen staatlichen Organisationen, Verbänden, Gemeinschaften und Akteuren des Marktes entwickeln. Die Beziehung betrifft Prozesse und Mechanismen zur Entscheidungsfindung und verbindet Akteure aus den unterschiedlichen gesellschaftlichen Sektoren (ebd., S. 34). In der vorliegenden Untersuchung soll die Kooperationsbeziehung zwischen dem forstlichen und dem sozialen Sektor analysiert werden. Kooperation zwischen Institutionen tritt vor allem dort auf, wo die Aufgabenerfüllung einzelner Institutionen an Grenzen stößt.

Für die erfolgreiche Kooperation müssen Merkmale bzw. Voraussetzungen gegeben sein, die im Folgenden beschrieben werden.

\subsubsection{Merkmale kooperativen Verwaltungshandelns}

Kooperation beinhaltet strukturelle, prozessuale und ergebnisbezogene Aspekte, die eine Mehrdimensionalität von Kooperation charakterisieren. Das Strukturelle Merkmal von Kooperation bezeichnet die Art der Zusammenarbeit von Partnern in Kooperationsbeziehungen. Als Voraussetzungen für eine erfolgreiche Kooperation gilt die wechselseitige Anerkennung der beteiligten Akteure im jeweiligen Interaktionsprozess als gleichberechtigt. Diese Forderung zieht die gleiche Verfahrens- und Entscheidungsrechte der Akteure im Kooperationsprozess nach sich. Auf einseitige Entscheidungskompetenzen und Handlungsmöglichkeiten wird verzichtet (auch wenn sie grundsätzlich bestehen) und stattdessen agieren alle Beteiligten als Verhandlungs- und Kooperationspartner mit gleichen 
Rechten und Pflichten. Das Prozessuale Merkmal kooperativer Aufgabenerfüllung bezeichnet die unmittelbare Interaktion von Kooperationspartner und setzt dialogische Kommunikation im Prozess voraus. Das zentrale Kennzeichen in Kooperationsbeziehungen besteht in der Beziehung der beteiligten und betroffenen Akteure, die durch "face-to-face" Kontakte geprägt ist. Weiterhin ist als prozessuales Merkmal die wechselseitige, direkte, sprachliche Kommunikation über Ziele, Interessen, Problemdefinitionen, Situationseinschätzungen, Kenntnisse und Werthaltungen der Akteure zu nennen, die aufeinander abzustimmen und in Einklang zu bringen sind. Das Ergebnisbezogene Merkmal von Kooperation beruht immer auf Verhandlungsprozessen. Hierbei soll zur kooperativen Aufgabenerfüllung auf eine freiwillige Einigung der beteiligten Akteure und Organisationen abgezielt werden. Demnach ist eine von allen Beteiligten akzeptierte Lösung der anstehenden Probleme angestrebt. Kooperation beschränkt sich zur Problemlösung nicht auf den Informations- und Meinungsaustausch, sondern zielt auf konkrete Lösungen ab. Das Ziel der Interaktions- und Kommunikationsprozesse ist eine von allen Beteiligten anerkannte gemeinsame Entscheidung. Nur wenn dieses Ziel gegeben ist, soll von kooperativem Verwaltungshandeln gesprochen werden (Benz, 1994, S. 37ff.).

\subsubsection{Entstehung von Kooperation}

Entscheidet sich eine Institution für eine kooperative Aufgabenerfüllung, so handelt es sich hierbei um das Ergebnis einer Strategieentscheidung. Strategien sind als Mittel zur Verfolgung von Zielen zu bezeichnen, während Egoismus, Altruismus und kooperative Orientierungen Zielrichtungen des Handelns darstellen. In egoistischen oder altruistischen Handlungsorientierungen bedarf es keiner Kooperation zur Verfolgung der eigenen Ziele, diese können durch einseitige Anordnungen oder Entscheidungen erreicht werden (Benz, 1994, S. 88). Es werden vor allem zwei wesentliche Handlungsorientierungen von Verwaltungen unterschieden, dogmatische und pragmatische Handlungsorientierungen. Unter dogmatischer Handlungsorientierung ist eine einseitig hoheitliche Handlungsweise zu verstehen, z.B. bei der Durchsetzung von rechtlichen Grundsätzen. D.h. in derartigen Konfliktsituationen wird es nicht zu einer kooperativen Aufgabenerfüllung kommen. Pragmatische Handlungsorientierungen fragen dagegen nach der Art der Konfliktregelung. Das Handeln wird vor einem pragmatischen Hintergrund auf die Bedingungen der Realisierbarkeit abgestellt, wobei die Wahl der Handlungsmittel wichtig ist. Denn die Strategiewahl eines pragmatischen Akteurs ergibt sich aus einer Optimierung von Zielen und Mitteln und ist nicht allein auf die Maximierung eines Ziels ausgerichtet. Akteure entscheiden sich deshalb in einer Konfliktsituation für eine Kooperation, zeigen also 
Kooperationsbereitschaft, wenn die Vor- und Nachteile aller verfügbaren Handlungsstrategien gegeneinander abgewogen sind. Einen Einfluss auf die positive Entscheidung für eine Kooperationsstrategie hat die Tatsache, dass die Akteure durch die Einigung mit anderen Akteuren besser gestellt sind als im Falle eigenständiger bzw. autonomer Interessensverfolgung. Um Entscheidungen überhaupt treffen zu können, ist es für Verwaltungen in bestimmten Situationen unumgänglich, Verhandlungen zu führen bzw. Kooperationen einzugehen. Die Erledigung oder Erfüllung von Aufgaben, die eine hohe Komplexität aufweisen, verlangen einen immensen Informations- und Kenntnisstand. Verwaltungen und Behörden sind häufig nicht in der Lage sich diesen umfassenden Wissensstand allein mit den verfügbaren personellen und sachlichen Mitteln zu verschaffen. So können durch die Verhandlungen mit Adressaten der anstehenden Entscheidungen für die Verwaltung notwendige Informationen gewonnen werden. Klienten der Verwaltung besitzen ebenfalls ein Kooperationsmotiv, was sich in dem Erhalt der eigenen Handlungsund Entscheidungsfähigkeit ausdrückt. Dies ist vor allem der Fall wenn diese auf staatliche Unterstützungsleistungen angewiesen sind. Für Verbände stellen Verhandlungsbeziehungen bzw. kooperative Beziehungen zu staatlichen Institutionen eine Bestandsgarantie dar. Auch Unternehmen sehen in der Kooperationsbereitschaft mit staatlichen Institutionen den eigenen Einfluss auf politisch-administrative Entscheidungen gesichert. Ein wesentlicher Grund Verhandlungen einzugehen bzw. Kooperationsbereitschaft zu zeigen, ist eine Unsicherheit über Ergebnisse und das Risiko des Scheiterns auf Seiten der Akteure. Diese Faktoren der Unsicherheit veranlassen Verwaltungen und deren Adressaten oftmals zum Eintritt in direkte Kommunikationsprozesse. Ein weiterer Grund, Kooperationen einzugehen, liegt in der Steigerung des Ansehens der beteiligten Akteure gegenüber der eigenen Klientel. Verwaltung können durch die Kooperationsbeziehungen ein bürgerfreundliches Image nach außen vertreten und Wirtschaftsunternehmen können das kooperative Verhalten werbewirksam einsetzen. Neben den Vorteilen, die kooperatives Verhalten für die Akteure bringt, sind Kooperationen auch immer mit Kosten verbunden, die eventuell Verhandlungen zwischen zwei Konfliktpartnern verhindern könnten. Die Kosten entstehen vor allem durch die zeitaufwendige Konsensfindung und den Einsatz von Kräften (Geld, Zeit, Personal) der Akteure. Für staatliche Institutionen sind die Kosten kooperativen Verhaltens jedoch meist günstiger als aufwendige Gerichts- und Widerspruchsverfahren mit den Adressaten ihrer Entscheidungen. Somit fällt die Entscheidung der Verwaltungen häufig zu Gunsten von Kooperation aus (Benz, 1994, S. 88ff.). 


\subsubsection{Interessen der Akteure}

Für die Kooperation zwischen Akteuren sind deren Interessen von entscheidender Bedeutung. Die Konstellationen von Interessen geben Auskunft darüber, wie sich inhaltliche Ziele der Akteure zueinander verhalten (Benz, 1994, S. 93). Diese können in zwei Klassen unterteilt werden, gleichgerichtet oder entgegengesetzt. Gleichgerichtete Interessen von Akteuren können sich identisch oder komplementär ausprägen. Identische Interessen von zwei Akteuren sind vollständig miteinander vereinbar und sind auf dasselbe Ziel ausgerichtet („was einer will, wollen auch die anderen“) (ebd.). Komplementäre Interessen von Akteuren haben das gleiche Ziel bzw. wirken in die gleiche Richtung, beziehen sich aber auf unterschiedliche Gegenstände, z.B. soziale Arbeit mir unterschiedlichen Personengruppen (ausländische Jugendliche und Langzeitarbeitslose) mit dem gemeinsamen bzw. sich ergänzendem Ziel der Integration dieser Gruppen. Im Gegensatz zu den ,harmonischen' Interessen, die eine Kooperation leicht machen bzw. die Bereitschaft zur Kooperation erheblich erleichtern, stehen entgegengesetzte Interessen der Akteure. Diese können als konkurrierend oder antagonistisch bezeichnet werden. Treffen konkurrierende Interessen von Akteure aufeinander, behindert die Handlung des einen Akteurs das Ausmaß der Interessensbefriedigung des anderen. Antagonistische Interessen schließen sich gegenseitig aus, da sie sich in der Zielerreichung gegenseitig behindern (ebd.).

Festzuhalten bleibt an dieser Stelle, dass bei identischen Interessen der Akteure eine Kooperation nicht notwendig ist, denn die Akteure verhalten sich aus eigenem Interesse den gemeinsamen Zielen entsprechend (ebd., S. 94). In Konfliktsituationen, d.h. bei Interessenkonstellationen in der die Handlungen von Akteuren nicht automatisch übereinstimmen, muss eine Entscheidung zwischen Kooperation und einseitiger Machtanwendung getroffen werden. Zu beachten ist an dieser Stelle auch, dass die Ausrichtung von Interessen allein noch nichts über die Kooperationsbereitschaft von Akteuren aussagt. Relevant für die Bereitschaft, eine Kooperation einzugehen ist dagegen das Ausmaß des Konflikts, d.h. die Differenz zwischen den angestrebten Zielen, die wiederum durch die Richtung der Ziele und ihre Bedeutung für den betreffenden Akteur bestimmt wird (ebd.). Ist ein Konflikt sehr stark und komplex, wird eine Einigung zwischen den Akteuren als unwahrscheinlich eingeschätzt und die Beteiligten rechnen eher mit einem Scheitern der Verhandlungen und treten gar nicht erst in diese ein. Eine Erfolgreiche Verhandlungslösung von Interessenskonflikten hängt zudem von der Zahl der involvierten Akteure ab, wobei hierfür die Unterscheidung zwischen bilateralen und multilateralen Konfliktbeziehungen bedeutsam ist. Bilaterale Interessenskonstellationen sind im Rechtsvollzug und bei der Leistungsvergabe im unmittelbaren Kontakt zwischen der Verwaltung (z.B. Ordnungsbehörden, Steuerverwaltung, Sozialverwaltung) und einzelnen Bürgern zu finden. Wobei multilaterale Beziehungen insbesondere bei Planungsprozessen, 
bei der Lösung von Verteilungsproblemen oder bei komplexen Genehmigungsverfahren vorliegen (Verwaltung - Adressaten - Auswirkung auf Dritte).

Für die Kooperation ist der Verhandlungsmodus von Bedeutung. Der Verhandlungsmodus gibt Auskunft über den Verhandlungsprozess bzw. -verlauf zwischen Akteuren, die zur Lösung eines Problems beitragen wollen.

\subsubsection{Verhandlungsmodus}

Zunächst lassen sich die Verhandlungsverläufe wie folgt unterscheiden: positionsbezogen, kompromissorientiert und verständigungsorientiert. Die Verhandlungsmodi unterscheiden sich im Ausmaß, wie Verhandlungsmacht von Akteuren in Kommunikation eingeht und zur Beeinflussung von Verhandlungsprozessen eingesetzt wird (Benz, 1994, S. 119).

\subsubsection{Positionsbezogene Verhandlungen}

Diese Form der Verhandlung ist darauf abgestellt, die Kontrahenten zur Einwilligung der eigenen Ziele zu bewegen. Eine Einigung durch ein solches Verhalten ist eher unwahrscheinlich. Trotzdem kann eine Lösung der Interessendivergenzen bei positionsbezogenen Verhandlungen gefunden werden, wenn sich der taktische Einsatz von Verhandlungsmacht der Akteure auf Gegenstände außerhalb des eigentlichen Verhandlungsthemas bezieht. Ein Beispiel hierfür wäre die Konzentration von Umweltverbänden auf ökologische Ausgleichmaßnahmen, wenn die Standortwahl des Unternehmens auf einem bestimmten schützenswerten Areal nicht mehr zur Disposition steht und eine Kooperation zu scheitern droht. Mit der Aufweitung des Verhandlungsthemas sind Zugeständnisse möglich, ohne die völlige Aufgabe der ursprünglichen Positionen der Akteure. Einigungen kommen in solchen Fälle durch Tausch zustande. Tausch bezeichnet die Verhandlungsform der Akteure, die ihre Position in Verhandlungen durchsetzen und dafür andere Akteure für ihr Nachgeben entschädigen. Dieser Tausch ist nur durchzuführen, wenn Änderungen von Verhandlungsinhalten und Argumentationsweisen möglich sind. Werden in Kooperationsbeziehungen Tauschprozesse aufgedeckt, gelten sie als Indiz für den Modus positionsorientierter Verhandlungen der Akteure, in denen die Position auf Kosten anderer Werte durchgesetzt und für die Erreichung der eigenen Ziele eine Gegenleistung erbracht wird (ebd., S. 120 ff.). 


\subsubsection{Kompromissorientierte Verhandlungen}

Die Basis der kompromissorientierten Verhandlungen zwischen Akteuren besteht darin, dass alle verhandelnden Akteure von ihren ursprünglichen Standpunkten abrücken und diese in Übereinstimmung bringen, indem ursprüngliche Ziele bzw. Forderungen nur teilweise realisiert werden. Sind Verhandlungspartner kompromissorientiert, akzeptieren diese eine Reduktion der eigenen Ansprüche und Forderungen zugunsten einer Einigung. Die kann nur bei einer hohen Kooperationsbereitschaft der Partner erreicht werden. Ein besonderes Merkmal dieses Verhandlungsmodus ist der Versuch der Teilnehmer die gemeinsame Basis ihrer individuellen Ziele auszuloten, die eine Einigung ermöglicht. Hierbei findet keine Verteidigung der einzelnen Standpunkte der Akteure statt, sondern eine Ermittlung der Konzessionsspielräume und Verhandlungsbereiche. Im Gegensatz zu den positionsbezogenen Verhandlungen, die von unterschiedlichen Interessen der Partner ausgehen, die nur durch Tauschprozesse zu einer Konfliktlösung beitragen, soll bei den kompromissorientierten Verhandlungen versucht werden, Interessensdivergenzen abzubauen. Die Kommunikationsstrategie in dieser Art von Verhandlung beruht auf Argumentationen, die Gemeinsamkeiten hervorheben, auf Streitvermeidung und auf Vertrauensbildung bzw. -stabilisierung. Der Prozess ist geprägt von inkrementellem Abtasten möglicher Verhandlungsbereiche, vergleichender Analyse von Standpunkten und Interessen, der Überzeugung, der Begründung relativer Vorteile und der schrittweisen Annäherung von Zielen und Interessen der Akteure. In diesem Kommunikations- und Verhandlungsmodus sind folgende Spielregeln von den Akteuren zu beachten, die zu einem erfolgreichen Ergebnis führen können. Zum einen sollten die Eingriffe in die Interessenbereiche der Verhandlungspartner minimal gehalten werden. Ein Entgegenkommen der Gegenseite kann nur in einem bestimmten Maß erwartet werden und Konzessionen müssen von allen Verhandlungsparteien erbracht werden. Die Verhandlungen in diesem Modus sind deutlich auf die Vermeidung von Konflikten ausgerichtet und hierzu einigen sich die Parteien meist auf den „kleinsten gemeinsamen Nenner“. Generell bedeutet die Einigung von Akteuren durch einen Kompromiss, dass die Verhandlungsteilnehmer auf eine vollständige Interessenverwirklichung zugunsten der Erhaltung einer Kooperationsbeziehung verzichten (Benz, 1994, S. 122ff.).

\subsubsection{Verständigungsorientierte Verhandlungen}

In diesem Verhandlungsmodus sollten Akteure aus sachlicher Überzeugung einem Ergebnis zustimmen, welches in Verständigungsprozessen zustande kommt. Die Voraussetzung 
hierfür ist eine weitestgehende Ausschaltung der Verhandlungsmacht von Akteuren. In diesen Verhandlungen gilt die reale (und nicht nur die formale) Gleichheit der Beteiligten als Ausgangsbasis, wobei sich die Akteure als Teilnehmer an einem kollektiven Lernprozess verstehen. Das Ziel dieser Form von Verhandlung ist die gemeinsame Konsensfindung in offener Diskussion und ein bewusster Verzicht auf Kompromisse. Siegrist (1970) definiert Konsens wie folgt:

„[...] das zwischen Interaktionspartnern aufgrund reflexiver Ko-orientierung erreichte Einverständnis“ (zitiert nach Benz, 1994, S. 125)

Der Verlauf der Verhandlungen ist von einem sachbezogenen Informations- und Meinungsaustausch zwischen diskussionsbereiten, selbstkritischen und lernwilligen Teilnehmern geprägt. Die Verständigung in diesen Verhandlungsprozessen setzt eine Anerkennung eines Wertepluralismus voraus und will keine Übereinstimmung von Werten oder Ideologien der beteiligten Akteure erreichen. Die Kooperation kann durch einen zweifachen Innovationsprozess als effektiv bezeichnet werden.

1. Entfalten die Beteiligten vollständig ihre "hermeneutisch - analytische Kompetenz", d.h. sie lernen sich gegenseitig umfassend zu verstehen und sich verständlich zu machen.

2. Tragen die Kommunikationsprozesse zur Überprüfung von Bewertungen bzw. Interessen und / oder zur Erfindung neuartiger Lösungen bei, die im Spektrum ursprünglicher Standpunkte und Verhandlungsbereiche der Akteure noch nicht angelegt waren.

Konsensfindung geht mit reflexiven Lernprozessen einher, die eine Veränderung von normativen und kognitiven Entscheidungsprämissen nach sich ziehen. Als Folge des Lernprozesses und der Veränderung von Entscheidungsgrundlagen steht eine ungezwungene Einwilligung in Entscheidungen, die ausschließlich durch akzeptierte Argumente motiviert ist. (Benz, 1994, S. 125ff.).

Nach der Darstellung von Interessen und Verhandlungsprozessen von Akteuren in Kooperationsbeziehungen soll die Innovation als weiterer Faktor beschrieben werden, der für die Kooperation entscheidend ist.

\subsubsection{Innovation}

Die Kooperationsbereitschaft von Akteuren variiert mit dem Innovationsgrad von Interessen, neben dem o.g. Ausmaß von Interessendivergenzen und der Zahl der zu beteiligten Akteure. Generell lässt sich die Aussage treffen, dass Akteure, die Veränderungen herbeiführen wollen, eher verhandlungswillig sind als jene, die bestehende Zustände verteidigen bzw. 
erhalten wollen. Reformen werden in der Regel auf dem Wege von Verhandlungen versucht zu realisieren, während Reformgegner Konfliktstrategien bevorzugen und keine Kooperation eingehen würden. Die kooperative Form der Aufgabenerfüllung gilt als wahrscheinlicher, wenn Interessen von Akteuren an einer Veränderung gegenüber Status-quo-Interessen überwiegen. Haben sich demnach Institutionen Innovationen, Reformen und Veränderungen zum Ziel gesetzt, sind sie bereit sowohl die Auflösung bestehender als auch die Ausbildung neuer Kooperationsformen einzugehen. Im Gegensatz hierzu sind Interessen, die eine Verteidigung des gegenwärtigen Zustandes vertreten, tendenziell weniger bereit aus bestehenden Kooperationsprozessen auszutreten und neue Kooperation zu forcieren (Benz, 1994, S. 98f.).

\subsubsection{Nutzen von Kooperation}

Der Nutzen der Kooperation für die einzelnen Institutionen ist eng mit den Faktoren der Entsehung von Kooperation verbunden. Der Nutzen der Zusammenarbeit zwischen Institutionen kann in der Steigerung der Position des Amtes oder der ausführenden Person liegen. Für das Amt kann die Kooperation mit anderen Institutionen eine Reputation bedeuten, die das Image der Institution in der Öffentlichkeit positiv beeinflusst. Die ausführende Person innerhalb der Institution hat ebenfalls einen Nutzen von der Kooperation, denn sie kann so die eigene Stellung in der Institution festigen und für kommende Zusammenarbeiten nutzen. Hierzu ist es notwendig, der für die Kooperation verantwortlichen Person, eine gewisse Autonomie in den Entscheidungsprozessen zu übertragen, damit die Kooperationsergebnisse auch entsprechend umgesetzt werden können (Benz, 1994, S. 183, 197). Der Informationsgewinn durch die Kooperation in verschiedenen Projekten kann zu einer Erhöhung des Machtpotentials der Institution beitragen. Informationen stellen ein Machtmittel der Fachverwaltungen dar, die so politische Maßnahmen gestalten können (Krott, 2001, S. 95). Informationen können das Handeln und die Entscheidungen von Akteuren beeinflussen. Zum einen geschieht dies über Aufklärung, was bedeutet, dass ein Akteur über einen Sachverhalt oder die Folgen seines Handelns faktenrichtig aufgeklärt wird. So entsteht eine umfangreiche Basis für dessen Entscheidungen. Wird ein Akteur durch Informationen in ein Abhängigkeitsverhältnis gebracht, ist dies Ausdruck von Machtausübung. Dies geschieht ebenso durch den Einsatz von Falschinformation und symbolischer Information (Informationen, die starke Gefühle verdichten), denn die Entscheidungen und das Handeln von Akteuren werden beeinflusst und gesteuert (ebd., S. 115f.). 
Den Ausführungen folgend, stellt der Informationsgewinn durch die Kooperation in Integrationsprojekten für die Forstverwaltungen eine Erhöhung des Machpotentials dar. Das forstliche Fachwissen kann durch das Wissen, wie Problemgruppen durch Projekte im Wald wieder in die Gesellschaft integriert werden können, erweitert werden. Dieses Wissen kann zur Gestaltung von Integrationsmaßnahmen innerhalb einer Stadt beitragen und stärkt den Einflussbereich der Forstverwaltung. Der Nutzen von Kooperation kann weiterhin durch die Erweiterung des Handlungsspielraumes, Erschließung finanzieller Ressourcen und Einflusssteigerung in gesellschaftliche Problembereiche lassen. Mantau (2001 a, b) und Mertens (2000) weisen ausdrücklich auf den schwindenden Handlungsspielraum und die Reaktion auf die gesellschaftlichen Belange der Forstverwaltung hin. Die Erschließung finanzieller Ressourcen durch die Kooperation schließt sich den Überlegungen an, denn die finanzielle Position der Forstverwaltungen in urbanen Zentren ist bedenklich, da die Produktionsfunktion des Waldes nur eingeschränkt genutzt werden kann und die Belastungen und Aufwendungen des Forstbetriebes hoch sind. Neue Produkte, die durch die Kooperation mit anderen Institutionen entstehen können, sollten sich daher positiv auf die Finanzlage der Forstverwaltungen auswirken (Gewinn erzielen).

Kontakte für weitere Projekte als Nutzen aus Kooperationsbeziehungen beschreibt Benz (1994). Die Wahrscheinlichkeit, dass eine Institution wiederholt in Kooperationsbeziehungen mit einer anderen Organisation tritt, erhöht sich mit einer erfolgreich abgeschlossenen Kooperation im Vorfeld. So entstehen Kontakte, die bei einer nachfolgenden Problemsituation wieder aufgenommen werden können und zu einer schnellen Lösung beitragen. Mit den Synergieeffekten als Nutzen von Kooperation sind Leistungen gemeint, die nur durch diese Kooperationsprojekte entstehen. Zu diesem Faktor werden die klassischen win-win-Situationen für die Institutionen gezählt, die einen Vorteil für beide Kooperationspartner bedeuten. Killich (2005) beschreibt dies als Zusammenarbeit, von der die Partner in gleichem Maße profitieren. Der Lerneffekt als Nutzen von Kooperationsbeziehungen stellt einen entscheidenden Faktor dar. Wie oben schon erwähnt, tragen die verständigungsorientierten Verhandlungen zu einem Lernprozess bei, der zu neuen und innovativen Lösungen von Problemen führen kann. Dieser Lerneffekt wird von Küker (2002, S. 129) als gemeinsames Grundwissen bezeichnet, das bei einer Kooperation entsteht, in die Kernkompetenzen der Akteure eingebracht und in einem Know-How-Transfer weitergegeben werden. Die Akzeptanz von Institutionen als Nutzen von Kooperationsbeziehungen hängt eng mit der Steigerung des Ansehens der Institutionen als Entstehung von Kooperation zusammen. Da das Ziel der Kooperation in einer gemeinsamen Aufgabenerfüllung besteht und dies von den Kooperationspartnern öffentlichkeitswirksam eingesetzt wird (Benz, 1994, S.90), kann davon ausgegangen werden, dass so die Akzeptanz der einzelnen Institutionen in der Öffentlichkeit gesteigert werden kann. 
Entscheidungen, die auf der Basis eines breiten Wissens- und Informationshintergrundes von Institutionen gemeinsam getroffen werden, finden in der Bevölkerung eher Vertrauen und so auch Akzeptanz.

Da davon ausgegangen wird, dass die Forstverwaltungen Kooperationsbeziehungen eingehen müssen, um einen Beitrag zur sozialen Integration zu leisten, soll die originäre Aufgabenwahrnehmung der Forstverwaltung Inhalt des nächsten Kapitels sein.

\subsection{Aufgabenwahrnehmung der Forstverwaltung}

Die staatlichen Forstverwaltungen sind durch die Bewirtschaftung des Waldes für betriebliche und forstpolitische Aufgaben zuständig. Hierzu gehören die Beratung und Betreuung von Waldbesitzern, die finanzielle Förderung und die Hoheit (Krott, 2001, S. 97). Kennzeichen für die Verwaltung dieses Sektors ist das Bürokratiekonzept, nach dem die politischen Programme streng rational in Problemlösungen vor Ort umgesetzt werden können (ebd., S. 98). Dies Konzept, mit den Merkmalen festgelegten Autoritätshierarchien und Kompetenzen, mit einer Regelgebundenheit und gleichförmigen Aufgaben- und Problemlösungen kann auf sich wandelnde Probleme eher langsam reagieren. Aus diesem Grunde sind Elemente bzw. Steuerungselemente des Managementkonzeptes auf die Problemlösung von Verwaltung zu übertragen. Kennzeichen dieses Konzeptes ist, neben der Qualitätssicherung durch Controlling, der Flexibilität und Leistungsanreizen für die Verwaltungseinheiten, das Angebot von kundenorientierten Verwaltungsprodukten (ebd., S. 98ff.). Die Verwaltungen sollten ihre Leistungen demnach aus Sicht der Betroffenen beschreiben und Produkte formulieren, die auf die Problemlösung des Kunden ausgerichtet sind. Zur Festlegung der Aufgaben bzw. Produkte ist die vorherige Ermittlung der Kundenwünsche erforderlich (ebd., S. 103f.). An diese Überlegungen schließen sich die Fragen an, ob soziale Probleme auch unter der Mitwirkung von Forstverwaltungen gelöst werden können? Kann die Forstwirtschaft durch die Produktentwicklung das soziale Potential des Waldes erschließen?

\subsection{Neue Produkte der Forstwirtschaft}

Aufgrund der Erläuterungen über die Aufgabenwahrnehmung der Forstverwaltung in den Großstädten entsteht die Diskussion über neue Produkte aus dem Wald. 
Um den Einfluss und die Position der Forstpartie zu stärken, müssen neue Produkte und Dienstleistungen geschaffen werden. Mantau (2001a) spricht von einer forstlichen Produktlücke, die es zu füllen gilt. Neue vermarktbare Produkte sollten aus den Funktionen des Waldes abgeleitet und der Forstwirtschaft zur Erweiterung des Arbeitsfeldes bzw. Handlungsraums dienen. Eines dieser neuen Produkte kann die Integration von sozialen Rand- bzw. Problemgruppen darstellen. Da der soziale Bereich einen Sektor mit der höchsten finanziellen Ausgabenbelastung des Staates darstellt, kann über forstliche Projekte zur Integration eine Entlastung der Ausgaben erreicht werden bzw. der Forstsektor kann von diesen Ausgaben partizipieren.

Ein innovatives Produkt, was der Wald bzw. die Forstverwaltung anbieten kann, muss folgenden Grundsatz für die erfolgreiche Platzierung beinhalten:

„Der Erfolg jedes Produktes hängt ganz stark zusammen mit seinem Umfeld: Je stärker ein Produkt in seinem Umfeld problemlösend wirkt, umso größer sind die Erfolgsaussichten." (aid infodienst, 2006)

Mertens (2000, S. 2f.) konstatiert, dass die Umwelt- und Erholungsleistungen als positive externe Effekte der Bewirtschaftung des Waldes angesehen werden können. Die Theorie der externen Effekte besagt, dass externe Effekte dann auftreten, wenn die Aktivität einer wirtschaftlichen Einheit eine andere Wirtschaftseinheit positiv oder negativ beeinflusst und dafür keinerlei Gegenleistungen (Bezahlung oder Entschädigung) vorgenommen werden. Es entstehen demnach Kosten (negative externe Effekte) oder Nutzen (positive externe Effekte), die als "extern“ zu bezeichnen sind, weil sie nicht der Wirtschaftlichkeitsrechnung der Verursacher zuzurechnen sind, sondern andere Wirtschafteinheiten bzw. die Gesellschaft betreffen. Demnach können die Umwelt- und Erholungsleistungen des Waldes als positive externe Effekte der Waldbewirtschaftung charakterisiert werden, da hier ein Nutzen entsteht, welcher der Gesellschaft zu Gute kommt. Die Gesellschaft stellt weithin Ansprüche an den Wald, die für einen Waldbesitzer als nicht ökonomisch zu bewerten sind, da hier keine Einnahmen erzielt werden können. Dies kann zu einer Unterversorgung der gesellschaftlich gewünschten Leistungen führen, wofür Marktversagen verantwortlich gemacht und das Eingreifen des Staates gefordert wird (Mertens, 2000, S. 3). Das Eingreifen des Staates wird über direkte $\mathrm{Ge}$ - und Verbote erreicht, die eine Bereitstellung oder Unterbindung von Leistungen durch die Waldbesitzer durchsetzen. Das Verhalten der Verursacher von positiven und negativen externen Effekten soll über Subventionen oder Steuern beeinflusst werden (Mertens, 2000, S. 3). Die Vermarktung von innovativen Umweltund Erholungsprodukten des Waldes bzw. des Forstbetriebes hängt von den Transaktionskosten ab, die bei der Vermarktung von Produkten entstehen. Hierzu sind Kosten zu zählen, die bei Such-, Informations- und Verhandlungsprozessen zwischen Anbietern und Nachfragern anfallen. Sind diese Kosten höher als die möglichen Einnahmen des Produktes, wird eine Vermarktung des Produktes aus ökonomischer Perspektive als 
nicht lohnend erachtet (ebd., S. 8). Bei einer steigenden Nachfrage der Gesellschaft nach Umwelt- und Erholungsprodukten und knapper werdenden Naturressourcen, könnte es für die Forstbetriebe lohnend werden, die Transaktionskosten für die Entwicklung neuer Produkte auf sich zu nehmen (ebd., S. 8). Die Nachfrage nach neuen innovativen Waldprodukten der Bevölkerung in Ballungsgebieten ist bei einer knappen Naturressource eine Chance für die ansässigen Forstbetriebe bzw. Forstverwaltungen, neue Produkte zu entwickeln und zu vermarkten. Diese Produktentwicklung findet verstärkt im Privatwald statt, da die Gewinnerzielung hier im Vordergrund steht. Die Waldleistungen als Produkte zu formulieren, fällt bei dieser Waldbesitzart leichter als im Kommunal- oder Staatswald. Dies liegt z.T. an der forstlichen Denkweise, dass öffentliche Güter nicht marktfähig seien (Mantau, 2001a). Öffentliche Güter besitzen folgende Eigenschaften:

„1. Ein Ausschluss von der Nutzung kann nicht durchgeführt werden.

2. Es besteht keine Rivalität im Konsum, d.h. die Nutzung durch eine Person schließt die weitere Nutzung durch andere nicht aus." (Mantau, 2001a, S. 7)

Öffentliche Güter können aber trotz ihrer Eigenschaften einen Raum für erlösorientiertes Handeln offen halten (ebd.). Als Beispiel kann hier die die Förderung eines Naturschutzgebietes durch ein Unternehmen angeführt werden. Das Unternehmen hat sich durch die Unterstützung des Gebietes das Recht erkauft, damit Werbung zu machen. Das Naturschutzgebiet bleibt weiterhin ein öffentliches Gut, aber das Recht auf Werbung mit der Unterstützung dieses Gebietes kann als ökonomisches Gut bezeichnet werden. Ein ökonomisches Gut zeichnet sich insbesondere dadurch aus, dass ein Defizit zwischen einem Bedürfnis und den zur Verfügung stehenden Mitteln besteht (hier: das Recht mit der Unterstützung des Naturschutzgebietes zu werben, schließt andere von der Nutzung dieses Rechts aus, so entsteht eine Rivalität in der Nutzung dieses Rechts) (ebd., S. 7). Nach diesen Ausführungen könnte die soziale Integration von Problemgruppen ein Produkt der Forstverwaltung darstellen. Dies Produkt ist von großem gesellschaftlichen Nutzen, dem sich die Forstwirtschaft nicht verschließen darf, sondern sich den gesellschaftlichen Anforderungen stellen muss (ebd., S. 7). Nach Mantau (2001b) muss in der Forstwirtschaft eine Entwicklung von der Waldfunktionenlehre zu einer Waldproduktlehre stattfinden. Hierfür sind vor allem zwei Aspekte erforderlich:

„1. Leistungen des Waldes aktiv zu gestalten und damit zugleich das Problem der NichtAusschliessbarkeit und der geringen Rivalität nicht hinzunehmen, sondern zu beeinflussen.

2. Institutionelle Rahmenbedingungen für die Vermarktung schaffen. Erst vorhandene Institutionen und vertragliche Lösungen ermöglichen den Marktzugang." (Mantau, 2001b, S. 12)

Dies stellt eine große, aber dennoch notwendige Herausforderung an den Forstsektor dar. Notwendig, da die Forstwirtschaft mit schwindenden Handlungsrechten zu kämpfen hat, weil die Umwelt- und Erholungsleistungen des Waldes die Handlungsrechte im Wald einerseits einschränken, auf der anderen Seite von anderen Sektoren übernommen werden und so den 
Handlungsbereich des Forstsektors eingrenzen (ebd.). Eine Produkterweiterung wirkt sich demnach auf den Handlungsspielraum des Forstes aus, der hierdurch ebenfalls eine Ausdehnung erfährt.

Im folgenden Kapitel sollen das Handlungsmodell und die Hypothesen zusammengefasst dargestellt werden.

\subsection{Forschungsansatz und Hypothesenformulierung}

Die theoretischen Grundlagen lassen folgenden Forschungsansatz ableiten:

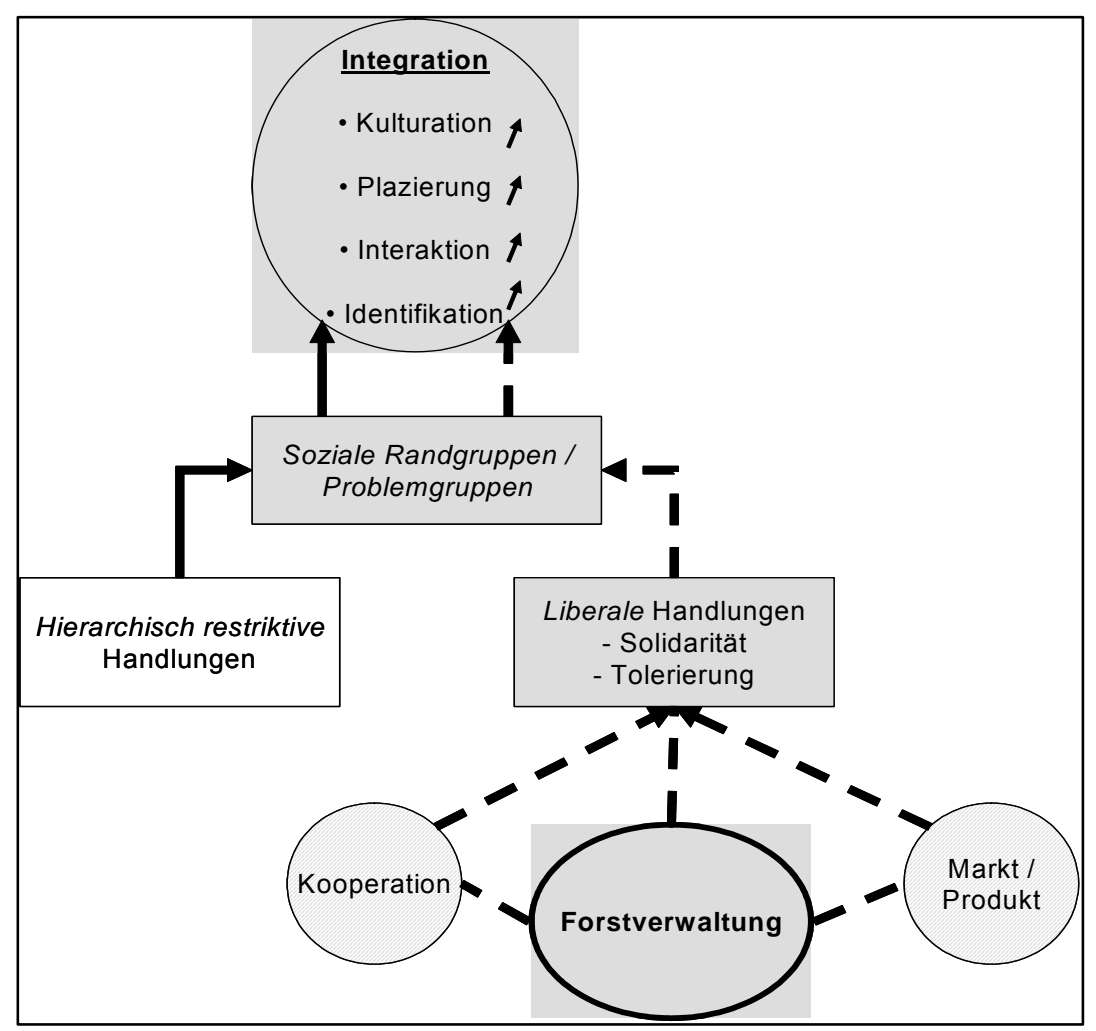

Abbildung 4: Forschungsansatz: Forstverwaltungshandeln für Liberale Integration

Die graphische Darstellung beinhaltet alle theoretischen Grundannahme des vorangegangenen Kapitels. Es soll untersucht werden, ob die Forstverwaltung zur sozialen Nachhaltigkeit und sozialen Integration beitragen kann. Es wird davon ausgegangen, dass der Wald ein soziales Potential besitzt, dies von der Forstverwaltung aber nur eingeschränkt genutzt wird. Diese eingeschränkte Nutzung ist vor allem auf das eingeschränkte Verständnis von sozialer Nachhaltigkeit zurückzuführen. Folgende Hypothese lässt sich aus den Überlegungen ableiten: 
Hypothese I: a) Der Wald in urbanen Räumen besitzt ein großes Potential für soziale Nachhaltigkeit und soziale Integration.

b) Aufgrund ihres eingeschränkten Verständnisses der sozialen Nachhaltigkeit erschließt die Forstverwaltung dieses Potential nur in geringem Umfang.

Das Forschungsprojekt soll weiterhin aufzeigen, ob die Forstverwaltung, durch die Strategieentscheidung in Kooperationsbeziehungen zu anderen Institutionen zu treten, dieses Potential des Waldes erschließen und so zur Integration von Randgruppen beitragen kann. Zudem ist davon auszugehen, dass die Forstverwaltung einen Nutzen aus diesen Kooperation ziehen kann, der sich in der Erschließung neuer Ressourcen und er Erweiterung des eigenen Handlungsspielraumes ausdrückt. Diese Überlegungen lassen folgende Hypothese zu:

Hypothese II: a) Die Forstverwaltung kann trotz ihres eingeschränkten Verständnisses durch Kooperation mit anderen Institutionen Lösungen für die Nutzung des Waldes zur sozialen Integration finden.

b) Die Forstverwaltung kann durch die Kooperation mit anderen Institutionen neue Ressourcen erschließen und den eigenen Handlungsspielraum erweitern.

Eine weitere Strategieentscheidung ist dem Forschungsansatz zu entnehmen, nämlich die Entwicklung des neuen Produktes „Integration“. Es soll untersucht werden, ob die Forstverwaltung durch diese Produktentwicklung das Potential des Waldes erschließen und zur Integration beitragen kann. Die Möglichkeit der Erweiterung des eigenen Handlungsspielraumes und der Erschließung neuer Ressourcen soll hier ebenfalls untersucht werden. Hierzu lässt sich eine dritte Hypothese formulieren:

Hypothese III: a) Die Forstverwaltung kann durch die Vermarktung des Produktes „Integration“ Lösungen zur Nutzung des sozialen Potentials des Waldes bieten. b) Die Forstverwaltung kann durch die Vermarktung des Produktes „Integration“ neue Ressourcen erschließen und den eigenen Handlungsspielraum erweitern. 


\section{Material und Methoden}

Zur Überprüfung der aufgestellten Hypothesen ist das empirische Datenmaterial von größter Bedeutung. Das folgende Kapitel beinhaltet die Auseinandersetzung mit der Erhebungsform der Daten, die konkrete Umsetzung der Erhebung und die Auswertungsmethoden, mit deren Hilfe das Material analysiert wird.

\subsection{Befragungsformen}

Im folgenden Kapitel soll die Methode der empirischen Datenerhebung erläutert werden. Die Untersuchung basiert auf der qualitativen Erhebungsmethode des teilstandardisierten Interviews. Um die übergeordneten Forschungsfragen zu beantworten und die aus der Theorie abgeleiteten Hypothesen zu belegen oder zu verwerfen, erschien die Anwendung der qualitativen Datenerhebung sinnvoll. Die Besonderheiten bzw. die Anforderungen der qualitativen Forschung an die Datenerhebung sind a) die Subjektbezogenheit, d.h. das Interesse an den „Sinndeutungen“ des Befragten, b) die Offenheit im Hinblick auf die Fragen, Antworten und Methoden und c) die Untersuchung in alltäglichen Situationen (Diekmann, 2000, S. 444). Ein Kritikpunkt, dem sich die qualitativen Methoden in der Sozialforschung häufig stellen müssen bzw. in der Vergangenheit mussten, ist die Behauptung, sie seien zur Hypothesenüberprüfung nicht geeignet, denn dies sei die Stärke der quantitativen Methoden.

Werden die von Diekmann (2000, S. 444) zusammengestellten Forschungsziele der qualitativen Erhebungs- und Auswertungsmethoden betrachtet, dann wird der o.g. Kritikpunkt widerlegt:

„1. Deskription empirischer Sachverhalte und sozialer Prozesse,

2. Aufstellung von Klassifikationen oder Typologien,

3. Gewinnung von Hypothesen am empirischen Material,

4. Prüfung von Forschungshypothesen." (ebd., S. 444)

Ein Kennzeichen der qualitativen Sozialforschung ist weiterhin die Untersuchung von Fallstudien und der wesentlich kleinere Stichprobenumfang als dies in der quantitativen Forschung üblich ist. Die qualitativen Methoden erlauben dagegen eine Vertiefung in der Datenerhebung, da z.B. der Interviewpartner in einer Befragung ausführlich zu Wort kommen kann und eine intensivere Analyse möglich ist, die auf statistische Kennzahlen verzichten kann (ebd. S, 445).

Im Folgenden wird der Einfachheit halber die männliche Form der interviewenden Person (Interviewer) verwendet, was keinerlei Wertung darstellen soll. Gleiches gilt für die Begriff ExpertIn und ForscherIn. Das Interview ist in der qualitativen Sozialforschung eine häufig angewandte Methode, die meist in Form eines Einzelinterviews stattfindet (Lamnek, 2005, S. 
342). Das Interview als Form der Befragung soll an die Alltagssituation des Befragten anknüpfen wodurch eine angenehme Gesprächssituation geschaffen wird. In der Befragung werden vor allem drei Formen des Interviews unterschieden. 1. das fokussierte, 2. das narrative und 3. das problemzentrierte Interview. Die fokussierte Interviewform hat einen bestimmten „Stimulus“ als Ausgangsbasis, der allen Befragten gleich ist. Hierbei stützt sich der Interviewer auf die Verwendung eines Leitfadens, der offene Fragen enthält und deren Reihenfolge nicht festgelegt ist. Weitere Besonderheiten bzw. Merkmale dieser Interviewform sind: a) alle Personen habe die gleiche konkrete Situation erlebt; b) eine Analyse der Situation ist im Vorfeld durch den Forscher vorgenommen worden, wobei Merkmale und Hypothesen erarbeitet wurden; c) durch die Situationsanalyse gelangt der Forscher zu dem Leitfaden, der alle wichtigen thematischen Aspekte des Interviews enthält; d) im Interview werden die subjektiven Erfahrungen der Personen bezüglich der Situation erhoben und die im Vorfeld definierten Hypothesen können überprüft werden, wobei das Interview offen bleibt für Antwortreaktionen der Befragten und neue Gesichtspunkte, die sich im Verlauf des Gespräches ergeben (ebd., S.44). Der Leitfaden hat im fokussierten Interview vor allem den Zweck, dass alle relevanten Aspekte und Themen angesprochen werden und eine Vergleichbarkeit der Antwortreaktionen unterschiedlicher Befragten gegeben ist. In der Interviewphase gelten vornehmlich folgende Prinzipien (ebd., S. 447):

1. Nicht-Beeinflussung: bedeutet, dass der Interviewer nicht direktiv in den Gesprächsfluss eingreifen soll und die Befragten die Möglichkeit haben, alle Informationen, die ihnen wichtig erscheinen, mitzuteilen.

2. Spezifizität: besagt, dass der Forscher versucht, Reaktionen auf bestimmte Details der erlebten Situation zu erlangen.

3. Erfassung eines breiten Spektrums: nimmt Bezug auf die thematischen Aspekte des Interviews, die angesprochen werden. Das Spektrum umfasst die erwarteten Reaktionen der Befragten, sowie die unerwarteten Reaktionen der Situation.

4. Tiefgründigkeit und personaler Bezugsrahmen: bezieht sich auf die Äußerung von Emotionen, die in Worten wie "gut" oder "hässlich“ ausgedrückt werden. Die Tiefgründigkeit einer Interviewsituation sollte nicht bei diesen Worten stehen bleiben, sondern die Reaktionen gezielt nachfragen.

Die Form des narrativen Interviews zielt auf erfahrungsnahe und subjektive Aussagen über Ereignisse und Situationen ab. Anwendung findet diese Interviewform, wenn Auskunft über z.B. besondere Phasen im Lebenslauf oder biographische Ereignisketten (Berufsverlauf, Ausbildungsverlauf etc.) erhalten werden soll. Im Vergleich zum fokussierten Interview sind die Anforderungen an den Leitfaden weniger strukturiert. Durch den Anstoß über eine einleitende Themenstellung soll der Befragten in den Erzählfluss gelangen und wird nur noch nicht-direktiv ermuntert fortzufahren. In dem Erzählfluss sollte der Befragte möglichst nicht 
unterbrochen werden, wobei Nachfragen nach Beendigung der Erzählung zulässig sind. Die Form des narrativen Interview ist nicht für jeden Interviewpartner geeignet, denn es kommt hierbei stark auf die "Erzählkompetenz" des Befragten an, die nicht bei jedem Interviewpartner gegeben ist (ebd., S. 449f.). So wurde die dritte Form des Interviews, nämlich das problemzentrierte Interview entwickelt. Hierbei kommt dem Interviewer eine aktivere Rolle zu als im vorhergenannten Beispiel. Fragen dürfen anhand des Leitfadens auch während der Erzählphase des Befragten gestellt werden ohne die Erzähllogik zu unterbrechen und der Situation angepasst. Hierdurch wird eine leicht höhere Strukturiertheit des Interviews erreicht, als das bei einem narrativen Interview der Fall ist (ebd., S. 451). Die einschlägige Literatur empfiehlt zur Erhebung von Daten, die über einen umfangreichen Sachverhalt Auskunft geben sollen, eine Kombination aus qualitativen und quantitativen Methoden.

In dieser Arbeit wurde nur die qualitative Erbungsform des Interviews gewählt, da es sich um ein neues und bis jetzt in der Forstwissenschaft unerforschtes Feld handelt und sich die Methode zum Einstieg in einen neuen Erkenntnisbereich sehr gut eignet. Die Interviewform (fokussiert, narrativ oder problemzentriert) ist im Gesprächsprozess dieser Untersuchung nicht trennscharf zu unterscheiden und enthält Teile aller drei Formen. Die Interviews wurden durch ein zuvor geführtes Telefonat thematisch eingegrenzt, so dass der Fokus des Gespräches auf den Projekten zur sozialen Integration liegt. Den Interviews liegt ein teilstrukturierter Leitfaden zugrunde, der größtenteils aus offenen Fragen besteht (s. Anhang). Im Interviewverlauf können Teile ausgemacht werden, die eher der narrativen Interviewform zuzuordnen sind, da in längeren Passagen der Befragte von seinen Erlebnissen und Erfahrungen in den Projekten berichtet. In diesen Phasen ist es vorgekommen, dass einige Fragen des Leitfadens, die im späteren Verlauf des Gespräches hätten gestellt werden sollen, schon behandelt wurden. Hier war dem Interviewer die Möglichkeit gegeben, Nachfragen oder vertiefende Fragen während des Erzählflusses zu stellen, was die problemzentrierte Form des Interviews widerspiegelt. Da es sich bei allen Interviewpartner der Untersuchung um VertreterInnen von Verwaltungen, Behörden oder Organisationen handelt, welche die Interessen und Erfahrungen der eigenen Organisationen vertreten, kann hier von einer besonderen Form des Interviews, nämlich dem Experteninterview gesprochen werden. Diese spezielle Form einer qualitativen Methode soll im folgenden Kapitel näher beschrieben werden. 


\subsubsection{Experteninterview}

Um die Fragestellung der vorliegenden Arbeit beantworten zu können und die Hypothese verifizieren oder falsifizieren zu können, finden Experteninterviews statt. Das Experteninterview kann als Form der Befragung als persönliches „Face-to-face“-Interview bezeichnet werden (Diekmann, 2000, S. 373). Interviews können nach der Form der Strukturierung unterschieden werden, d.h. von „vollständig strukturiert" bis hin zu „unstrukturiert, offen“. Vollständig strukturierte Interviews haben den Vorteil der Objektivität und Reliabilität, wobei mit Objektivität gemeint ist, dass die Befragung bzw. das Interview von zwei verschiedenen Personen durchgeführt werden könnte und wiederholt das gleiche Ergebnis erzielt wird. Reliabilität bezeichnet die Verlässlichkeit oder Zuverlässigkeit der erhobenen Daten bzw. gibt das Maß der Reproduzierbarkeit der Meßergebnisse an (ebd. S. 217 u. 374). Eine vollständige Standardisierung eines Interviews birgt die Gefahr, dass der Interviewer keine zusätzlichen Informationen neben denen der Antwortkategorien enthält. Die Form des standardisierten Interviews gilt dann als zweckmäßig, wenn ein erhebliches Vorwissen über die soziale Situation, die erforscht werden soll, vorhanden ist (ebd., S. 374).

Das Experteninterview, als spezielle Form des Interviews, findet in der Sozialforschung häufig Anwendung. Hierbei wird auf das Wissen spezifischer Akteure zurückgegriffen, die für das Thema oder das Fachgebiet als kompetent erachtet werden (Bogner u. Menz, 2002a, S. 7). Die Vorteile des Experteninterviews liegen in der Datengewinnung in der Explorationsphase des Forschungsvorhabens, denn zu diesem Zeitpunkt ist das Projekt noch wenig strukturiert und vernetzt, so dass mit relativ geringem Aufwand ein hohes Maß an qualitativ hochwertigen Daten erhoben werden kann. Weiterhin kann das Experteninterview den einfachen Zugang zu schwierigen sozialen Untersuchungsfeldern, wie z.B. tabuisierte Themen liefern. Experten werden in dem meisten Fällen als Vertreter einer Gruppe von Akteuren gesehen und interviewt, welche die Erfahrungen und das Wissen dieser spezifischen Gruppe wiedergeben. Diese Vorteile sind vor allem forschungsökonomischer Natur, da sie den Forschern Investitionen in zeitlicher, organisatorischer und finanzieller Hinsicht sparen. Gelangt der Forscher bei dem Experteninterview an eine Person, die eine Schlüsselposition innehat, kann so der gesamte Feldzugang erleichtert werden, denn die befragte Person verweist auf weitere Personen, die als potentielle Gesprächspartner in Frage kommen könnten. Mit dem Vertrauensbonus des Experten kann der Forschungsprozess wesentlich erleichtert werden (ebd., S. 8). Als weiterer Vorteil dieser Methode gilt die Motivation der Experten an der Untersuchung teilzunehmen, denn die Relevanz von Forschung muss nicht erst erläutert werden, da sich die Interviewpartner meist auf einer bildungsgleichen Ebene bewegen. Auf den ersten Blick ermöglicht diese Ebene eine ,symmetrische Kommunikationsbeziehung' (ebd., S. 9). Durch die genannten Vorteile avancierte das Experteninterview zu einer beliebten 
Erhebungsmethode, da sie schnell, objektiv und relativ unproblematisch Daten liefert. Die Expertise ist besonders gefragt, wenn gesellschaftliche Zusammenhänge und politische Steuerungsprozesse untersucht werden sollen (ebd., S. 10). In der empirischen Sozialforschung schlägt der Methode des Experteninterviews Kritik entgegen. So wird das Experteninterview nur als Einstieg in das Forschungsfeld, in der explorativen Phase, für sinnvoll erachtet. Es kann zudem nur zur Vertiefung eines vorwissenschaftlichen Wissens dienen, zur Hypothesengenerierung und gilt somit als „unreife Vorstufe zum eigentlichen Forschungsprozess“ (ebd., S. 18). Die anklingende Geringschätzung dieser Erhebungsform begründet sich vor allem aus der wenig ausgeprägten theoretischen und methodischen Fundierung (ebd., S. 20). Gerade diese Methodenkritik versuchen Bogner und Menz (2002b) zu entschärfen, indem sie zunächst die Formen des Experteninterviews nach deren erkenntnisleitenden Funktion getrennt betrachten. Die Trennung erfolgt zwischen dem explorativen, dem systematisierenden und dem theoriegenerierenden Experteninterview (ebd., S. 37). Das explorative Experteninterview dient, wie oben schon angedeutet, einer ersten Orientierung im Forschungsfeld, der Problemdefinition des Forschers, der Hypothesenformulierung oder zur Erstellung eines abschließenden Leitfadens. Hierbei können die Experten sowohl zur Zielgruppe der Untersuchung zählen, als auch zur Informationsquelle über eine bestimmte Zielgruppe. Die Form der Gesprächsführung in den explorativen Interviews ist offen, wobei die zentralen Themen in einem Leitfaden vorstrukturiert sein sollten. Vergleichbarkeit, Vollständigkeit und Standardisierbarkeit der Daten sind keine vordergründigen Ziele dieser Form des Experteninterviews. Das systematisierende Experteninterview zielt ebenfalls auf das exklusive Handlungs- und Erfahrungswissen der Experten ab. Eine systematische und lückenlose Information von den Experten soll erlangt werden, wobei die Experten als Ratgeber zu sehen sind, die über objektive Tatbestände aufklären, die eigene Sicht zu bestimmten Themen erläutern und über Wissen verfügen, welches den Forschern ohne das Interview verschlossen bliebe (ebd., S. 37). Dem Interview sollte ein ausdifferenzierter Leitfaden zugrunde liegen, denn die Vergleichbarkeit der Daten steht bei dieser Form des Experteninterviews im Vordergrund, im Gegensatz zum explorativen Interview. Im theoriegenerierenden Experteninterview dienen die Experten nicht mehr allein zur Gewinnung von Information und Aufklärung, sondern tragen zur Entwicklung einer Theorie, z.B. über die gesellschaftlichen Zusammenhänge bei. Dies wird möglich, da die Experten über Wissensbestände, Weltbilder und Routinen verfügen, diese wiederum durch deren organisatorisch-institutionelle Anbindung abgesichert sind und sich daraus Typologien und Konzepte ableiten lassen. Durch die Vergleichbarkeit der Daten sind die Forscher in der Lage, die Informationen generalisierend zu interpretieren und daraus eine Theorie zu entwickeln (induktive Theoriebildung) (ebd., S. 38f.). Die Ausführungen über die unterschiedlichen Formen des Experteninterviews betrachtend, kann 
für die vorliegende Arbeit festgehalten werden, dass die Form des systematisierenden Experteninterview abgewendet worden ist, da es sich um ausdifferenzierte Leitfadengespräche handelte, um die Daten für die Analyse möglich vergleichbar zu erheben. Im Vordergrund der Untersuchung stand, neben der Vergleichbarkeit der Daten, die Gewinnung objektiver Information durch das Erfahrungs- und Handlungswissen der interviewten Experten.

\subsubsection{Wer gilt als Experte}

Meuser und Nagel (2002, S. 72) betonen, dass in Experteninterviews der organisatorische oder institutionelle Hintergrund bzw. die Eingebundenheit der befragten Person wichtig ist und von den individuellen Orientierungen und Einstellungen der Person abzutrennen ist. Experten sind als Teil des Handlungsfeldes, welches den Forschungsgegenstand darstellt, zu betrachten. Sie fungieren demnach nicht als externe Gutachter, die Stellung zu dem Handlungsfeld nehmen. Dieser Typus von Experten wurde in die vorliegende Untersuchung einbezogen, denn (fast) alle befragten Personen sind Teilnehmer an den sozial integrativen Projekten. Die Frage, die sich logisch anschließt ist, wie wird eine Person zu einer Expertin bzw. einem Experten. Dies soll im Folgenden kurz erläutert werden. Zwei allgemeine Faktoren weisen Personen als Experten aus:

- „wer in irgendeiner Weise Verantwortung trägt für den Entwurf, die Implementierung oder die Kontrolle einer Problemlösung oder

- wer über einen privilegierten Zugang zu Informationen über Personengruppen oder Entscheidungsprozesse verfügt." (Meuser und Nagel, 2002, S. 73)

Diese Charakteristika von Experten beschränken die Auswahl von Interviewpartnern nicht nur auf die oberen Ebenen in Organisationen, sondern beziehen sich ebenfalls auf die Ebenen, wo die Entscheidungen vorbereitet und umgesetzt werden (ebd., S. 74). Die Erfahrungen und das Wissen der Experten, die im Interview erhoben werden, können als repräsentativ für die Organisation oder Institution der die Befragten angehören, gewertet werden (ebd.). Experten sind in dieser Studie als Zielgruppe zu sehen, da sie Informationen über das eigene Handlungsfeld geben sollen (ebd., S. 75). Die in der Studie ausgewählten Experten erfüllen die hier aufgeführten Kriterien. Es handelt sich um Personen, die Schnittstellen der Institution oder Organisation bekleiden, sie verfügen über detaillierte Informationen und Entscheidungskompetenz in Bezug auf die untersuchten Projekte oder sind direkt der ausführenden Ebene angeschlossen. Die Tabelle 11 soll einen Überblick der Experten geben: 
Tabelle 11: Ausgewählte ExpertInnen nach Fachbereichen

\begin{tabular}{|c|c|}
\hline Fachbereich & Befragte ExpertInnen \\
\hline Forst & $\begin{array}{ll}\text { - } & \text { Forstamtsleiterlnnen } \\
\text { - } & \text { Revierleiterln }\end{array}$ \\
\hline Grünflächen & $\begin{array}{ll}- & \text { Fachbereichsleiterln } \\
\text { - } & \text { Leiterln der Personalabteilung }\end{array}$ \\
\hline Sozial & $\begin{array}{ll}- & \text { Leiterlnnen gemeinnütziger Einrichtungen (Arbeitsförderung, } \\
\text { - } & \text { Suchtkrankenhilfe, Straffälligenhilfe) } \\
\text { - } & \text { SozialarbeiterInnen } \\
\text { - } & \text { Leiterln Jugenamt } \\
\text { - } & \text { Leitarbeiterlnnen des Sozialamtes umweltpädagogischen Verein } \\
\text { - } & \text { Leiterln Jugendförderung } \\
\text { - } & \text { Suchbeauftragte der Stadt } \\
\text { - } & \text { Mitarbeiterln Behindertenverein }\end{array}$ \\
\hline Städtische Ämter & $\begin{array}{ll}\text { - } & \text { Stadtrat } \\
\text { - } & \text { Leiterln Ferienspiele }\end{array}$ \\
\hline
\end{tabular}

Experteninterviews kommen dort zum Einsatz, wo auf das Wissen bestehend aus Erfahrungsregeln von Personen, die wiederum das Funktionieren sozialer Systeme bestimmen, abgezielt wird (Meuser und Nagel, 2002). Die Schwierigkeit eines Experteninterviews besteht darin, zu beurteilen, ob die Befragten die „Wahrheit" sagen. Es können demnach in den Interviews beschönigte oder zu lückenhafte Erfahrungsberichte entstehen. Dadurch, dass die Befragten durchaus damit rechnen können, dass ihre KollegInnen ebenfalls befragt werden, ist davon auszugehen, dass sie „wahrheitsgemäße“ Aussagen treffen. Ein gewisses Maß an Subjektivität ist selbstverständlich nicht auszuschließen.

\subsubsection{Leitfadengestützte Befragung}

In der vorliegenden Arbeit wird das teilstandardisierte Interview zur Erhebung der Daten herangezogen. Bei dieser Form des Interviews wird zum größten Teil mit offenen Fragen gearbeitet, die dem Interview die Möglichkeit geben, das Gespräch in Teilen selbst zu strukturieren (Kromrey, 1994, S. 286). Der besondere Vorteil dieser Befragungsmethode liegt in der Chance, bestimmte Themen genauer nachzufragen und Sachverhalte intensiver und tiefergehend zu erfassen (ebd.). Als weiteren Vorteil des Leitfadeninterviews nennt Marotzki (2006, S. 114) die Sicherstellung, dass interessierende Aspekte im Verlauf des Gespräches auch wirklich angesprochen werden. Dies gewährleistet wiederum die Vergleichbarkeit der 
Interviews, denen der gleiche Leitfaden zugrunde liegt. Das Erstellen eines Leitfadens erfordert im Vorfeld Kenntnisse über das Forschungsobjekt, denn hier sollen relevante Themen näher beleuchtet werden. Der Leitfaden gilt allgemein als Gedächtnisstütze für den Interviewer und gibt einen Orientierungsrahmen vor, der dem Interviewten die Möglichkeit geben soll flexibel auf die Fragen zu reagieren. Aus diesem Grund sollte der Leitfaden in der Interviewsituation flexibel gehandhabt und nicht als starres Konstrukt gesehen werden. Für das Experteninterview betonen Meuser und Nagel (2002) die Verwendung eines Leitfadens für das Experteninterview als unerlässlich für den Erfolg des Gespräches. Da sich Forscher schon im Vorfeld mit dem Themenbereich bei der Erstellung des Leitfadens auseinandersetzen, treten sie als kompetente Gesprächspartner auf, geben dem Gespräch die gewünschte Richtung und werden nicht in Themen abdriften, die nicht von Interesse sind. Nur der Leitfaden bzw. die Auseinandersetzung des Forschers mit dem Thema ließe die Offenheit des Interviewverlaufes und eine „lockere, unbürokratische“ (ebd., S. 78) Unterhaltung zu. Der Leitfaden kann auch zu Schwierigkeiten führen, wenn der darin gewählte Sprachstil nicht dem des Experten entspricht. Befindet sich ein Forscher in einer solchen Situation, sollte er sich, um das Gespräch zu einem positiven Abschluss zu bringen, auf die Sprache des Interviewten einlassen.

In der Methode des Experteninterviews können neben der sprachlichen Barriere weitere Schwierigkeiten auftreten, die zum Misslingen des Interviews beitragen. Zum einen kann der Experte das Interview blockieren (ebd., S. 78). Diese Blockade kann auftreten, wenn die Person fälschlicherweise als Experte ausgewählt wurde, sich im Thema nicht auskennt oder sich auf eine formale Ebene der Themenbehandlung zurückzieht. All diese Aspekte signalisieren dem Forscher, dass er sich nicht auf die noch anstehenden Themen konzentrieren, sondern sich ganz auf den Experten einlassen sollte. Ein Experteninterview kann weiterhin misslingen, wenn die Experten die Forscher zu „Mitwissern“ machen und zwar im negativen Sinne. Die Situation des Gespräches wird ausgenutzt, um eigene Themen anzubringen, welche die Experten in diesem Moment beschäftigen. Es werden z.B. Interna der Behörde oder Verstrickungen im Arbeitsumfeld ausgebreitet und sind von der Seite des Interviewers schwer einzufangen bzw. zu stoppen. Wechseln Experten in Interviews häufig die Rollen, d.h. sprechen sie mal als Experte und mal als Privatmensch und ziehen Beispiele aus diesem Umfeld heran, kann der Forscher nichts über das zu erforschen Themenfeld in Erfahrung bringen und das Interview kann als misslungen betrachtet werden (ebd., S. 78f.). Die Informationen aus den o.g. misslungenen Interviews sind sehr dürftig und können in die Auswertung kaum aufgenommen werden. Zum Gelingen eines Experteninterviews tragen dagegen die Neugierde des Experten auf die Untersuchung und die damit zusammenhängenden Themen bei. Ebenso der Respekt und die Kompetenz des Forschers und das Interesse am Gedankenaustausch seitens des Experten (wobei das Gespräch 
hierdurch auch negativ beeinflusst werden kann, indem der Experte nur die Bestätigung seiner Position durch den Forscher sucht). Die Form des rhetorischen Interviews liegt für den Forscher zwischen Ge- und Misslingen des Interviews. Hierbei referiert der Experte über sein Wissen in einem Vortrag und falls einige Themen, die den Forscher interessieren, angeschnitten werden, kann der Forscher die Informationen verwenden. Der „Referent“ kann ebenso die Themen nicht behandeln und so ist das Gespräch für den Forscher zwecklos (ebd., S. 79).

\subsubsection{Datenerfassung bzw. Datenaufzeichnung}

Ein wichtiger Punkt der qualitativen Interviews ist die Aufzeichnung des Gesprächsverlaufs. Die Datenerfassung ist ein essentieller Bestandteil des Forschungsprozesses, denn nur so kann die Güte und die Interpretation der Daten gewährleistet sein (Lamnek, 2005). So gilt es, das Interview in einer geeigneten Form aufzuzeichnen. Die beste Variante der Aufzeichnung stellt die Audiovisuelle Datenaufzeichnung dar, denn so können Gesten und Mimik der Befragten in die Interpretation einfließen und erweitern den Erkenntnisraum. In der vorliegenden Untersuchung wurden die Gespräche mit Hilfe eines digitalen Diktiergerätes aufgezeichnet, was für die Forschungsfrage adäquat erschien. Das Einverständnis der Befragten wurde vor Beginn des Gespräches eingeholt und die Anonymität der Daten und deren Verarbeitung versichert. Alle ausgewählten Gesprächspartner, bis auf eine Ausnahme, stimmten der Aufzeichnung zu. Das Gerät wurde in der Mitte zwischen Befragten und Interviewerin plaziert und fand nach einigen einleitenden Sätzen keinerlei Beachtung durch die Befragten. Das Gespräch wurde in den meisten Fälle durch die Vorstellung des Forschungsthemas und den Weg dorthin, die institutionelle Anbindung und den Werdegang der Forscherin eingleitet. So wurden die Experten zunächst informiert, häufig deren Neugierde geweckt und das Gespräch in Gang gesetzt (vgl. Meuser u. Nagel, 2002). Die Bereitschaft zur Interviewteilnahme wurde schon in dem Telefonat mit den einzelnen Akteuren deutlich, welches den ersten Kontakt herstellte und schon einige Vorinformationen auf beiden Gesprächsseiten lieferte. Die Reaktionen auf die Frage nach einem ausführlicheren Gespräch wurde von fast allen angesprochenen Personen positiv aufgenommen. Es soll an dieser Stelle erwähnt werden, dass vor allem die sozialen Einrichtungen, die sich mit sozialen Problemgruppen beschäftigen, sehr offen für ein Interview waren und häufig geäußert haben, dass sie froh seien, wenn ein Interesse an ihrer Arbeit bestünde und sie darüber berichten könnten. So war der Zugang zum Forschungsfeld und dessen Akteuren in den Fallstudien von keinen unüberwindlichen Hindernissen geprägt. 


\subsubsection{Fallstudien}

Die Untersuchungsanordnung der vorliegenden Arbeit stützt sich auf die Fallstudie. Lijphart (1971, S. 691) weist als Vorteil der Fallstudie die Konzentration auf einen Fall auf, der intensiv betrachtet und analysiert werden kann, auch bei relativ beschränkten Ressourcen der Forscher. Dieses Charakteristikum der Fallstudie benennt auch Kromrey (1994, S.426), nämlich dass interessante Fälle besonders intensiv befragt, beschrieben und analysiert werden. In der quantitativen Sozialforschung werden Fallstudien vornehmlich zur Exploration des Forschungsfeldes eingesetzt, wobei sie in der qualitativen Forschung einen höheren Stellenwert besitzen (ebd., S. 427). Lijphart (1971, S. 691) weist sechs unterschiedliche Typen von Fallstudien aus:

„1. Nichttheoretische Fallstudien;

2. Interpretative Fallstudien;

3. Hypothesen - generierende Fallstudien;

4. Theorie - bestätigende Fallstudien

5. Theorie - schwächende Fallstudien

6. Abweichende Fallstudien." (Übersetzung durch Verf.)

Im Folgenden sollen die Eigenschaften von Interpretativen Fallstudien kurz erläutert werden. Die interpretative Fallstudie wird gewählt, da der Fokus eher auf dem interessanten Fall liegt und weniger auf der Formulierung einer generellen Theorie. Hierin ähneln sich die ersten beiden Typen von Fallstudien. Der große Unterschied der interpretativen Fallstudie besteht in der Anlehnung an bzw. Nutzung von theoretischen Aussagen. Die Fallstudie soll hierbei keinen Beitrag zur empirischen Generalisierung liefern, sondern lediglich den Fall beleuchten. Auch Krott (1990, S. 88) verweist auf die Gewinnung von Wissen in einem ganz speziellen Fall mit Hilfe der Fallstudienmethode. Die Besonderheit der interpretativen Fallstudie liegt nach diesen Ausführungen in den Faktoren, die durch die angewandte Theorie vorgegeben und in den Fallstudien betrachtet werden. Hieraus ergibt sich eine direkte Überprüfung der Theorie, d.h. die Aufdeckung von Schwächen und Stärken der Theorie und versetzt die Forscher in die Lage neue Hypothesen zu entwickeln, die eine größere Aussagekraft besitzen (ebd., S. 88). Durch die verbesserten Hypothesen ist es der Fallstudienmethode möglich, diese auch empirisch zu testen. Dies kann in drei Varianten geschehen:

1. Zunächst können Plausibilitätstests anhand des empirischen Materials durchgeführt werden. Diese Tests sind nicht so aussagekräftig wie die Tests der vergleichenden Analyse, dennoch sind sie einfach durchzuführen und für den ersten Schritt der empirischen Theorieprüfung unerlässlich.

2. Durch Fallstudienketten kann die theoretische Aussagekraft von Fallstudien erhöht werden. Die Kette entsteht durch die erste Fallstudie, die als heuristische Fallstudie angelegt ist und Erfahrungen und Erkenntnisse für eine weiterführende Fallstudie 
liefert. Die um das Wissen erweiterte Studie kann daraufhin den Plausibilitätstests unterzogen werden und durchdringt so ein Politikfeld auf einer breiten empirischen Grundlage.

3. Die theoriegeleitete Fallstudienauswahl stellt die dritte Art dar, Hypothesen empirisch zu testen und zudem die theoretisch anspruchvollste, da hierbei theorieentscheidende bzw. theoriebildende Fälle untersucht werden. Die Besonderheit dieser Fallstudien liegt in der Überprüfung ganzer Theorien bzw. Theoriegebäuden, die zu einer Erweiterung, Verwerfung oder Annahme der angewandten Theorie führt (ebd., S. 88f.).

Die Methode der Fallstudie kann also einen entscheidenden Beitrag zur empirischen Überprüfung von theoriegeleiteten Hypothesen leisten. Durch diese Überprüfung ist sie ebenfalls in der Lage, die Theorie kritisch zu bewerten, d.h. Stärken und Schwächen der Theorie aufzuzeigen und zur Theoriebildung beizutragen. Hildenbrand (1995, S. 257) unterscheidet ebenfalls unterschiedliche Ansätze der Fallstudie. Zum einen differenziert er das Hypothesengenerierungs-Modell, wobei der Fall dazu dient, Hypothesen zu Beginn des Forschungsprozesses $\mathrm{zu}$ erhalten, die aufgrund von statistischer Repräsentativität generalisiert werden können. Dem gegenüber steht das Sozialreportage-Modell, in deren Fällen keine generellen Aussagen getroffen werden, sondern die Fälle als selbsterklärend gelten. Das Subsumtions-Modell nimmt eine gewisse Zwischenstellung ein und wird häufig in der qualitativen Sozialforschung angewendet. Die Besonderheit liegt hierbei in der Erhebung von Fällen, dessen Material dann z.B. durch die aus der Theorie abgeleiteten Begriffe und Hypothesen ausgewertet und erklärt wird. Eine vierte Variante der Fallstudie lässt sich als FallrekonstruktionsModell bezeichnen, bei der es darauf ankommt, zu rekonstruieren, wie ein Fall seine spezifische Wirklichkeit im Kontext allgemeiner Bedingungen konstruiert hat. Die Fallstudie wurde für diese Untersuchung als Methode aus den o.g. Gründen ausgewählt. Dem Forschungsvorhaben liegen eine Theorie bzw. ausgewählte Theorien zugrunde, woraus die Hypothesen abgeleitet worden sind. Die Hypothesen gilt es nun mit Hilfe der Fallstudien empirisch zu überprüfen und plausibel zu erläutern. Dieses Verfahren würde der ersten Variante der Hypothesenüberprüfung entsprechen (s.o.). Zudem handelt es sich um eine Methode, die organisatorisch einfach und ressourcensparend durchzuführen ist (ebd., S. 90). Die Auswahl für die geeigneten Fälle dieser konkreten Untersuchung basiert auf der wissenschaftlichen Fragestellung und der Praxis der sozialen Integration. Sie bezieht sich also auf die Projekte zur sozialen Integration mit Waldbezug und den daran teilnehmenden Institutionen und Organisationen. Da der Fokus dieser Untersuchung auf den urbanen Zentren liegt, werden die Fallstudien nach 
den Städten benannt, in denen die Experteninterviews stattgefunden haben. So können im Weiteren die Projekte nach den jeweiligen Städten betrachtet und analysiert werden.

\subsection{Auswahl der Städte}

Um die Frage nach den integrativen Projekten und die Zusammenarbeit verschiedener Institutionen in diesen Projekten zu beantworten, sollten Vertreter der Institutionen der Untersuchungseinheit angehören. Institutionen der Untersuchungseinheit sind hierbei diejenigen, deren Aufgabe die Verwaltung und das Management der Wald- und Grünflächen ist und die sozialen Institutionen, die sich mit der Unterstützung und Hilfe von sozialen Problemgruppen beschäftigen. Konkret sind dies: Forstverwaltungen (Forstämter oder Abteilungen der für den Wald zuständigen Ämter), Sozialämter, Jugendämter, Umwelt- und Grünflächenämter, gemeinnützige Einrichtungen. In empirischen Untersuchungen ist zudem die Grundgesamtheit der in die Untersuchung einzubeziehender Objekte festzulegen. Werden alle Fälle oder Objekte in die Untersuchung aufgenommen, handelt es sich um eine Vollerhebung. Erstreckt sich die Untersuchung hingegen nur auf eine Teilmenge bzw. Auswahl der Grundgesamtheit, wird von einer Teilerhebung gesprochen (Kromrey, 1994, S. 187). Die Auswahl der Untersuchungsobjekte (aus einer Grundgesamtheit von 78 Städten (Deutscher Städtetag, 2004)) für die vorliegende Studie fand nach festgelegten Kriterien statt, wobei als erstes ausschlaggebendes Kriterium die Größe der Stadt zu nennen ist. Untersucht werden sollten nur Städte, die mehr als 100.000 Einwohner haben und demnach als Großstädte bezeichnet werden (Klassifizierung nach W. Christaller, zitiert nach www.egeography.de). In die Untersuchung wurden folgende Städte einbezogen: Göttingen, Kassel, Freiburg i.Br., Frankfurt a.M., Hamburg, Düsseldorf, Berlin, Nürnberg. Auswahlkriterium neben der Stadtgröße war die Waldfläche im Stadtgebiet bzw. im direkten Einzugsgebiet der Stadt. Im Folgenden sollen die Strukturdaten der einzelnen Städte in einer Tabelle zusammengefasst werden: 
Tabelle 12: Strukturdaten der Städte (Quellen: Websites der aufgeführten Städte)

\begin{tabular}{|c|c|c|c|c|c|}
\hline Stadt & Einwohner & $\begin{array}{c}\text { Waldfläche in } \\
\text { ha }\end{array}$ & Ausländer & $\begin{array}{l}\text { Empfänger von } \\
\text { Sozialleistungen }\end{array}$ & Arbeitslose \\
\hline Göttingen & $\begin{array}{c}129.686 \\
(03 / 07)\end{array}$ & $3.491(04 / 07)$ & $12.652(03 / 07)$ & $13.777(11 / 06)$ & $9.168(05 / 07)$ \\
\hline Kassel & $\begin{array}{c}191.652 \\
(12 / 06)\end{array}$ & $2.306(01 / 07)$ & $25.415(12 / 06)$ & k.A. & $13.184(05 / 07)$ \\
\hline Freiburg i.Br. & $\begin{array}{r}217.547 \\
(01 / 07)\end{array}$ & 6,480 & $30.728(01 / 07)$ & $10.557(2004)$ & $11.552(12 / 06)$ \\
\hline Frankfurt a.M. & $\begin{array}{c}651.583 \\
(2005)\end{array}$ & 3.823 (2005) & $161.592(2005)$ & $39.578(2004)$ & $34.217(06 / 05)$ \\
\hline Hamburg & $\begin{array}{l}\text { 1.74 Mio } \\
(08 / 05)\end{array}$ & 4.700 & $\begin{array}{c}244.520 \\
(06 / 05)\end{array}$ & k.A. & 92.000 \\
\hline Düsseldorf & $\begin{array}{l}581.858 \\
(12 / 06)\end{array}$ & $2.418(12 / 05)$ & $\begin{array}{l}106.961 \\
(12 / 05)\end{array}$ & $6.649(12 / 05)$ & $33.852(04 / 07)$ \\
\hline Berlin & 3.4 Mio $(07 / 06)$ & $16.054(12 / 05)$ & $\begin{array}{l}460.555 \\
(12 / 05)\end{array}$ & $275.691(12 / 04)$ & $\begin{array}{l}277.211 \\
(10 / 06)\end{array}$ \\
\hline Nürnberg & $\begin{array}{l}499.237 \\
(12 / 05)\end{array}$ & $3.113(06 / 06)$ & 88.978 (2004) & $49.715(12 / 05)$ & $33.830(06 / 05)$ \\
\hline
\end{tabular}

Die Städte wurden aufgrund der Größe und der Waldfläche ausgewählt, wobei die Waldfläche im Eigentum der jeweiligen Stadt ist und demnach als Kommunalwald zu bezeichnen ist. Der erste Schritt des Auswahlverfahrens fand in Form eines Telefonates statt, bei dem zunächst das ansässige Forstamt bzw. zuständige Behörde für den Wald angesprochen wurde. In diesem ersten Gespräch wurde das Anliegen des Forschungsvorhabens kurz erläutert und die Frage nach integrativen Projekten im Wald oder mit Waldbezug gestellt. Wurde diese Frage mit "nein“ beantwortet, d.h. es finden keine Projekte zur Eingliederung von Problemgruppen in die Gesellschaft unter Einbeziehung des Waldes statt, wurde die Stadt nicht in die Untersuchungseinheit aufgenommen. Somit stellt das Nicht-Vorhandensein von integrativen Projekten mit Waldbezug das Ausschlusskriterium für die Untersuchung dar. Handelt es sich in Untersuchungen um Teilerhebungen, können über die Untersuchungseinheiten hinaus Aussagen über die Gesamtheit der Fälle angestrebt werden (Kromrey, 1994, S. 188). Dies kann als Repräsentationsschluss geschehen, wobei hier die Ergebnisse der Teilerhebung als Hypothesen oder generelle Aussagen auf die Grundgesamtheit verallgemeinert werden. Der Inklusionsschluss hingegen testet eine vorhandene Theorie oder Hypothese anhand der Ergebnisse der Teilerhebung bzw. Stichprobe (ebd.). In der hier vorliegenden Studie handelt es sich um eine Teilerhebung wobei Theorien und daraus abgeleitete Hypothesen getestet werden sollen, die Aussagen 
über die Grundgesamtheit (alle integrativen Projekte für Problemgruppen mit Waldbezug) zulassen (Inklusionsschluss).

In qualitativen Interviews unterliegt die Auswahl der Befragten nach Lamnek (2005, S. 384ff.) zudem fünf Kriterien.

1. Typisierung statt Repräsentativität: besagt, dass für das Erkenntnisinteresse keine Zufallsstichprobe erhoben werden muss, sondern die Fälle gezielt für die Befragung ausgewählt werden können.

2. Verzerrungen: sollten durch das gewonnene Vorwissen der Forscher und deren Interessen vermieden werden.

3. Die Auswahl der Personen: sollte nicht aus dem Bekanntenkreis des Interviewers erfolgen, da dies zur Verzerrung der Ergebnisse bzw. Erkenntnisse führt.

4. Offenheit: sollte der Forscher beweisen, indem er Fälle in die Untersuchung einbezieht, die von seiner ersten Vorstellung abweichen und die Auswahl sukzessive erweitern.

5. Der Forscher sollte auch über die inhaltliche Offenheit verfügen, die er auf die Auswahl der Gegenstände der Untersuchung anwenden sollte.

\subsection{Erstellung des Fragenbogens und Kategoriensystems}

Als wesentlicher Bestandteil der Untersuchung ist die Entwicklung des Fragebogens für die teilstrukturierten Experteninterviews zu bewerten. Um die o.g. Hypothese beantworten zu können, wird ein Fragebogen entwickelt, der die Hauptbestandteile der Theorien enthält, auf die sich das Forschungsprojekt stützt.

Für die Analyse des Datenmaterials ist die Erstellung eines Kategoriensystems von großer Bedeutung. Dieses Kategoriensystem kann nach Mayring (1997, S. 74f.) theoriegeleitet oder induktiv erstellt werden. Theoriegeleitete Kategorien sind den theoretischen Vorüberlegungen zur Beantwortung der Hypothesen entnommen. Die induktive Kategorienableitung kann als empiriegeleitet bezeichnet werden. Die Kategorien werden hierbei direkt aus dem Datenmaterial abgeleitet. Dies stellt eine Besonderheit der qualitativen Inhaltsanalyse, die in dieser Untersuchung zur Anwendung kommt, dar. Durch die Analyse des Materials können im Verallgemeinerungsprozess Kategorien direkt aus den Gesprächen gebildet werden (Mayring, 1997, S. 74f.). Hierbei wird kein Bezug zu einer Theorie hergestellt. In dieser konkreten Untersuchung wurde das Hauptaugenmerk auf die Kategorienbildung aus den verschiedenen Theorien gelegt. Dennoch wurde das Kategoriensystem in den ersten Analyseschritten um Kategorien erweitert, die sich aus den Interviews als beachtenswert ergeben haben, z.B. durch Mehrfachnennung der Experten. 
Dieses Prozedere wurde gewählt, um eine möglichst genaue Abbildung der Realität und eine detailgetreue Interpretation zu gewährleisten. Die Formulierung der Kategorien bzw. die Ableitung der Kategorien aus den Theorien sollte folgende Forderungen erfüllen. Die Kategorien sollten das Datenmaterial umfassend wiedergeben. Weiterhin müssen sie zu allen weiteren Kategorien abgegrenzt sein, eine Zuordnung einer Kategorie darf nicht zweifelhaft sein oder die Einordnung in eine andere bedingen (Mayring, 1997). Das Kategoriensystem, welches die Basis der Analyse für die vorliegende Untersuchung bildet, wird im Folgenden dargestellt. Die Operationalisierung der Fragen, die das Gerüst des Interviewleitfadens darstellen, ist im Anhang I zu finden. Die Fragen des Leitfadens sind aus den Theoriebestandteilen entnommen. Im Anhang II finden sich ebenfalls alle Definitionen der Kategorien.

Um die Übersicht des Kategoriensystem bzw. der Ableitung der Kategorien aus der Theorie zu gewährleisten, werden die Kategorien in der Reihenfolge der drei Hypothesen dargestellt.

\subsubsection{Hypothese I - Integration}

Der Einstieg zur Bearbeitung der Hypothese I gelingt über einen deskriptiven Teil des Kategoriensystems, der allgemeine und grundlegende Angaben und Informationen aufzeigt. Die Kategorie Kontakte und Verhältnis lässt Schlüsse auf die Berührungspunkte der Institutionen mit den Problemgruppen zu, weist Konflikte und die Art der Konflikte aus und soll zeigen, wie die Institution auf die Konflikte aufmerksam wird. Zudem gibt die Charakterisierung von Randgruppen Einblick in die Zusammensetzung, Eigenheiten und Besonderheiten dieser Gruppen. Die Darstellung der Kategorien und deren Codes erfolgt über die Ebenen (1. bis 4.), die im Analyseprogramm verwendet werden.

\begin{tabular}{|c|}
\hline $\begin{array}{c}\text { Kontakte und Verhältnis } \\
\text { Berührungspunkte (mit wem + wie) } \\
\text { Konflikt }\end{array}$ \\
Art des Konflikts \\
Interessenskonflikt \\
Aufenthalt an Orten \\
Umgang miteinander \\
Lärm \\
Nachbarschaftsverhältnis \\
Einhaltung von Regeln \\
Kommunikation \\
Müll \\
Hunde \\
Sonstiges \\
Charakterisierung von Randgruppen
\end{tabular}

Abbildung 5: Deskription

Nach den einleitenden, beschreibenden Informationen werden nun die Kategorien aufgezeigt, die zur eigentlichen Hypothesenüberprüfung nötig sind. Da im deskriptiven Teil 
ein Schwerpunkt auf den Konflikten mit Problemgruppen liegt, sollte zunächst geklärt werden, mit welchen Instrumenten die Institutionen zu einer Konfliktlösung beitragen, worin die Ansprüche von Randgruppen bestehen und ob diese Ansprüche von der Institution erfüllt werden können.

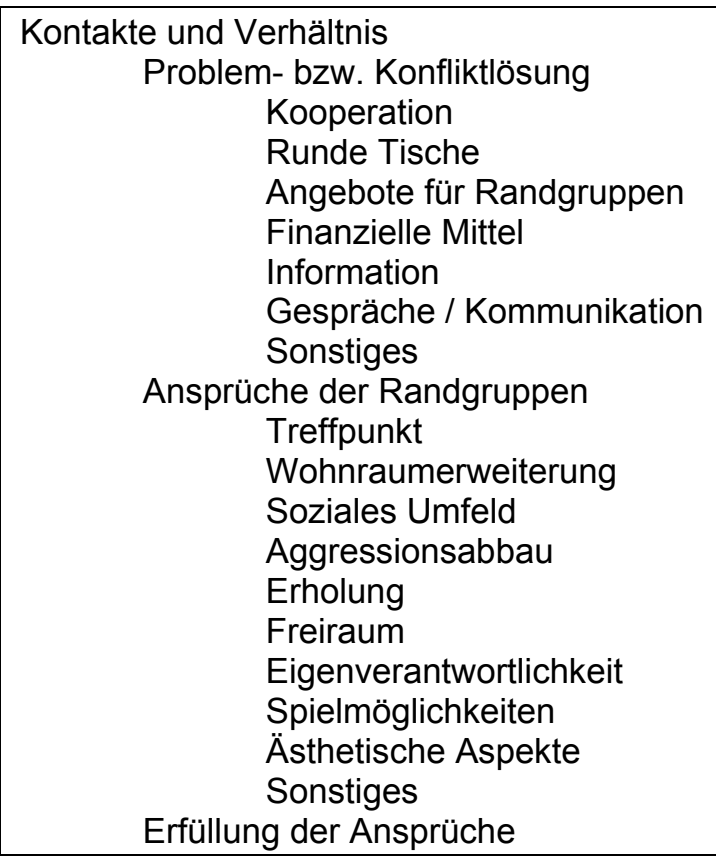

Abbildung 6: Kontakte und Verhältnis

Die Instrumente (Codes der 2. Ebene) der Problem- und Konfliktlösung (Abb. 6) sind aus den vorangegangenen Ausführungen zur sozialen Arbeit mit unterschiedlichen Randgruppen hervorgegangen (s. Kap. 1.3.1). Die Codes der 2. Ebene zu den Ansprüchen der Randgruppen sind deduktiv in der Analyse aufgenommen worden.

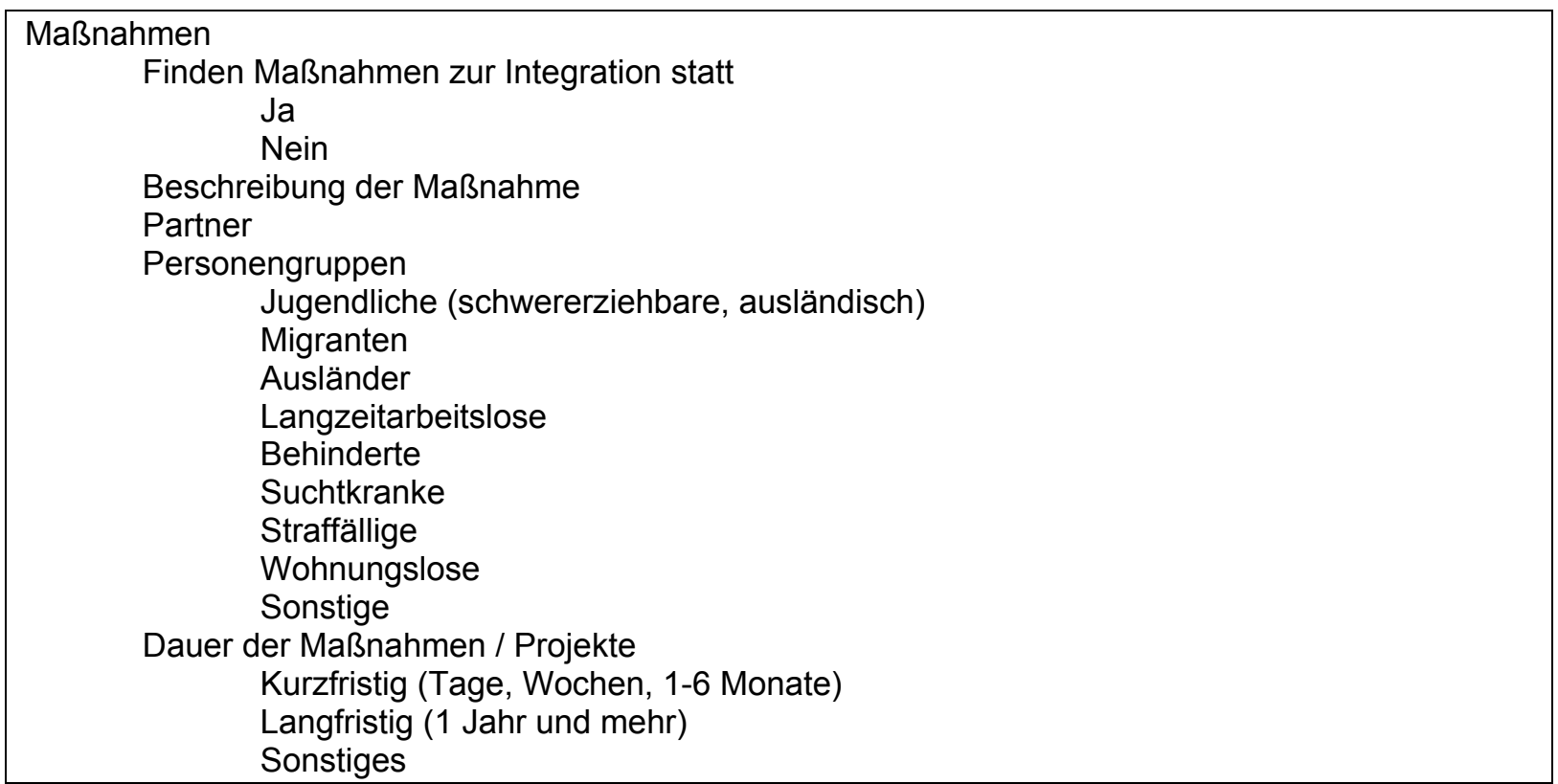


Die oben stehende Abbildung legt den Schwerpunkt auf die Kategorie Maßnahmen. Hier sollen zunächst beschrieben werden, ob überhaupt Maßnahmen oder Projekte im Wald mit Rand- bzw. Problemgruppen stattfinden. Nach der Beschreibung der Projekte und Nennung der Projektpartner, erfolgt die Einteilung für welche Personengruppen die Projekte durchgeführt werden. Die Codes der Kategorie sind wiederum dem Kap. 1.3.1 entnommen, die im Vorfeld der Untersuchung festgelegt worden sind, da das weite Feld der Problemgruppen eine Eingrenzung erfahren musste. Die Dauer der Projekte, als Code 1. Ebene, soll die Qualität der Maßnahmen überprüfen. Bei der Festsetzung der Zeiteinheiten wurde sich nach den Vorgaben aus den Projekten der Sozialen Arbeit (z.B. Arbeitsförderungsmaßnahmen) gerichtet.

Der letzte Teil der Hypothese I setzt sich direkt mit der Integration auseinander. Es werden in der Kategorie Integration Codes aufgenommen, die einen Beitrag zur sozialen Integration durch die Maßnahmen und Projekte nachweisen sollen.

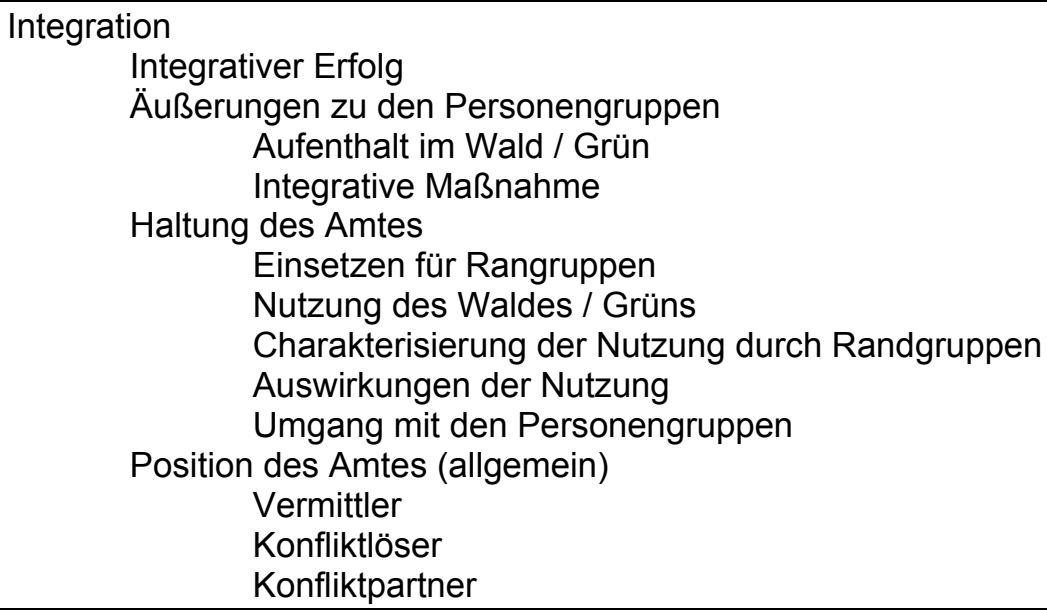

Abbildung 8: Integration

Die Kategorie Integration und deren Codes wird als Operationalisierung der Faktoren Solidarität mit Anderen und Tolerierung der Anderen des „Liberalen Modells der sozialen Integration“ verstanden. Da sich diese Aspekte auf Handlungen beziehen und diese über Äußerungen zu Werten und Haltungen gemessen werden können, wurde hier sowohl die missbilligenden oder respektvollen und auch anerkennenden Aussagen zum Aufenthalt im Wald und auf Grünflächen aufgenommen. Da es sich bei den Experten immer um Vertreter von Organisationen und Institutionen handelt, werden zudem die Äußerungen beachtet, die stellvertretend als Meinung, Haltung oder Wertung des Amtes getätigt werden. Können in der Analyse Äußerungen gefunden werden, die einen Schwerpunkt in der Kritik und Abneigung gegenüber Randgruppen und deren Nutzungen der Flächen setzen, kann nicht von einem Beitrag zur sozialen Integration dieser Gruppen gesprochen werden. Denn hier findet keine Behebung von Diskriminierung und Hilfe für Bedürftige statt, sondern Ausgrenzung und Unverständnis wird gefördert. Genau in die entgegengesetzte Richtung lassen sich die 
Aussagen und Äußerungen der Experten interpretieren, die sich positiv und respektvoll den Problemgruppen, den Maßnahmen und den Nutzungen der Flächen gegenüber äußern. Diese Äußerungen sind als Beitrag zur sozialen Integration dieser Personengruppen zu werten. Auch die Einordnung der Institution, basierend auf deren Aussagen, in die Codes Vermittler, Konfliktlöser und Konfliktpartner sind Ausdruck des Beitrages zur sozialen Integration. Tritt das Amt als Vermittler in Problemsituationen zwischen Konfliktparteien auf, zeigt Verständnis für die Gruppen und kann zur Problemlösung beitragen, kann dies einen positiven Einfluss auf die Integration der Personen nehmen. Tritt das Amt zunehmend als Gegenspieler in einem Konflikt mit Randgruppen auf, wird hier eher ein negativer bzw. kein Beitrag zur sozialen Integration zu erwarten sein.

\subsubsection{Hypothese II - Kooperation}

Aus den theoretischen Merkmalen, die Kooperation beschreiben, lassen sich zur Auswertung der Experteninterviews folgende Kategorien und Codes ableiten:

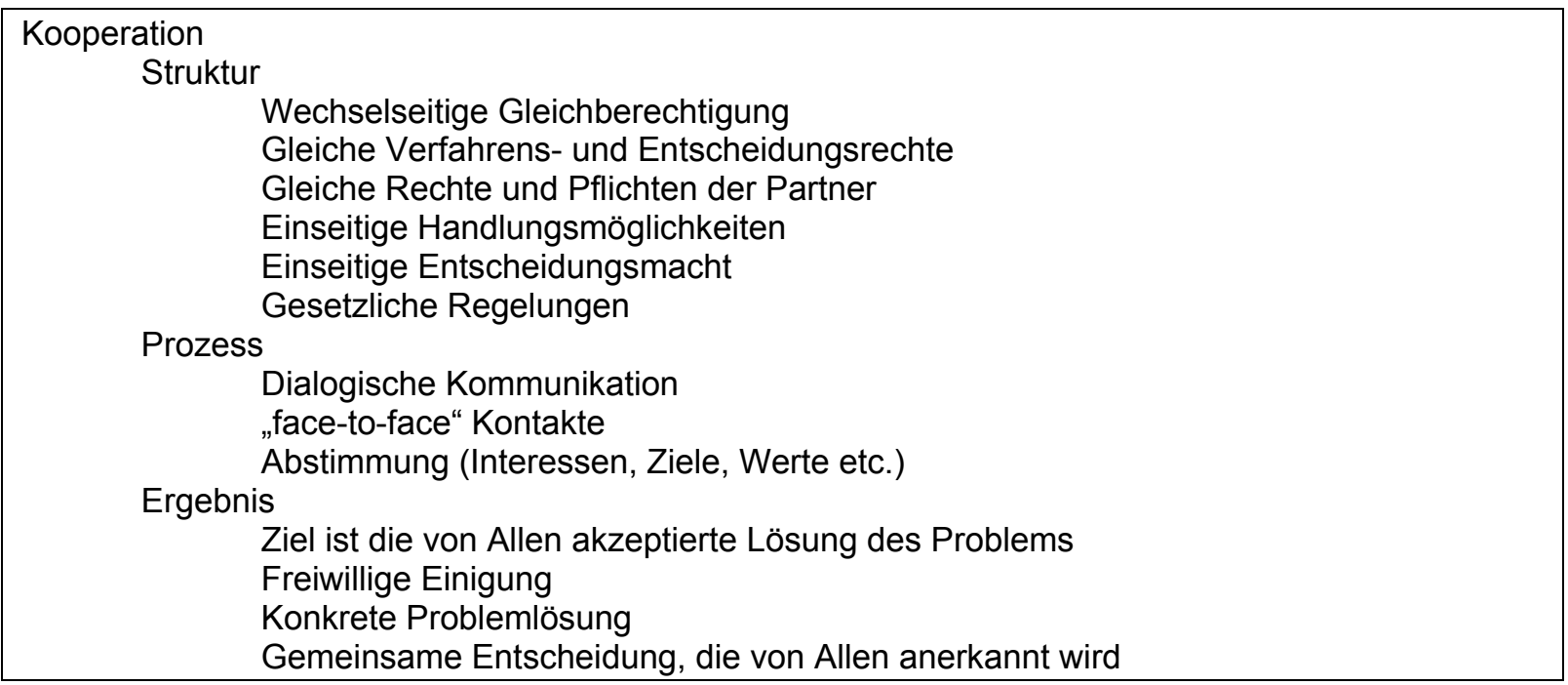

Abbildung 9: Mehrdimensionalität von Kooperation

Die Mehrdimensionalität von Kooperation ist von den Merkmalen geprägt, welche die Struktur der Zusammenarbeit, die praktische Zusammenarbeit der Partner und die Ergebnisse der Zusammenarbeit beschreiben. Ist von Kooperation die Rede, muss auch die Entstehung dieser speziellen Interaktionsform beachtet werden. Für das Forschungsprojekt ist es besonders interessant zu erfahren, von wem die Kontaktaufnahme vor Beginn der eigentlichen Kooperation ausging. Hierzu wird in das Analyseraster unter der Kategorie Kooperation ein Code Kontaktaufnahme mit vier Ausprägungen aufgenommen (s. Abb. 10) 


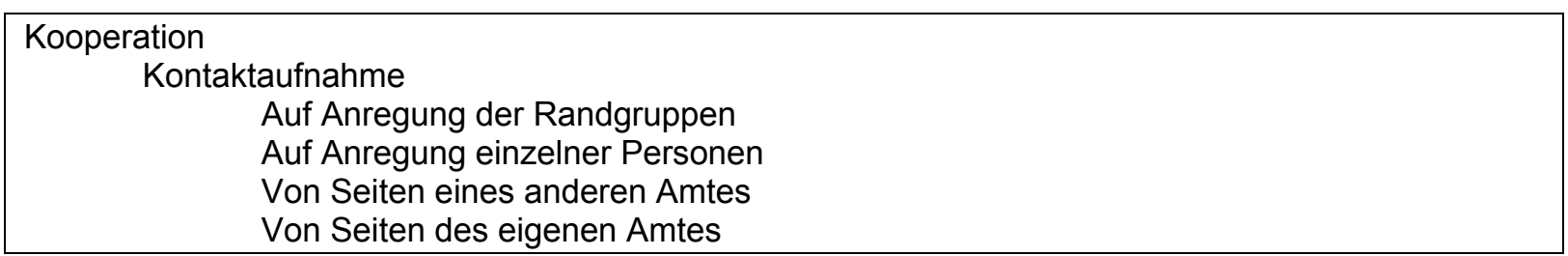

Abbildung 10: Kontaktaufnahme

Da der Hypothese II die Annahme zugrunde liegt, dass die Forstverwaltung die Kooperation nur mit minimalem Einsatz betreibt, werden die Ausprägungen ,von Seiten des eigenen Amtes' und ,von Seiten eines anderen Amtes' berücksichtigt. Diese sollen zeigen, ob die Initiative zur Kooperation in Integrationsprojekten eher von Seiten der sozialen Institutionen oder auch von Seiten der Forstverwaltung ergriffen wird. Die Ausprägung ,von Seiten der Randgruppen' soll aufzeigen, ob die Institutionen erst auf die Nachfrage oder das Vorhandensein eines Problems reagieren und dann zur Problemlösung eine Kooperation eingehen. Die Ausprägung ,auf Anregung einzelner Personen' soll zeigen, ob Kooperationsprojekte zur Integration wesentlich vom Aktionismus Einzelner abhängen. Benz (1994) beschreibt, dass Kooperationen häufig von einer Person initiiert werden, da diese entweder eine Schlüsselposition (Grenzstelle) innerhalb der Institution und die Entscheidungskompetenz innehat oder die Kooperation nutzt, um die eigene Position in der Institution zu festigen bzw. sichern. Welche weiteren Aspekte zur Entstehung von Kooperation beitragen, soll im folgenden Abschnitt dargestellt werden.

Aus diesen theoretischen Grundlagen für die Entstehung von Kooperation lassen sich folgende Analysekategorien für das Datenmaterial ableiten:

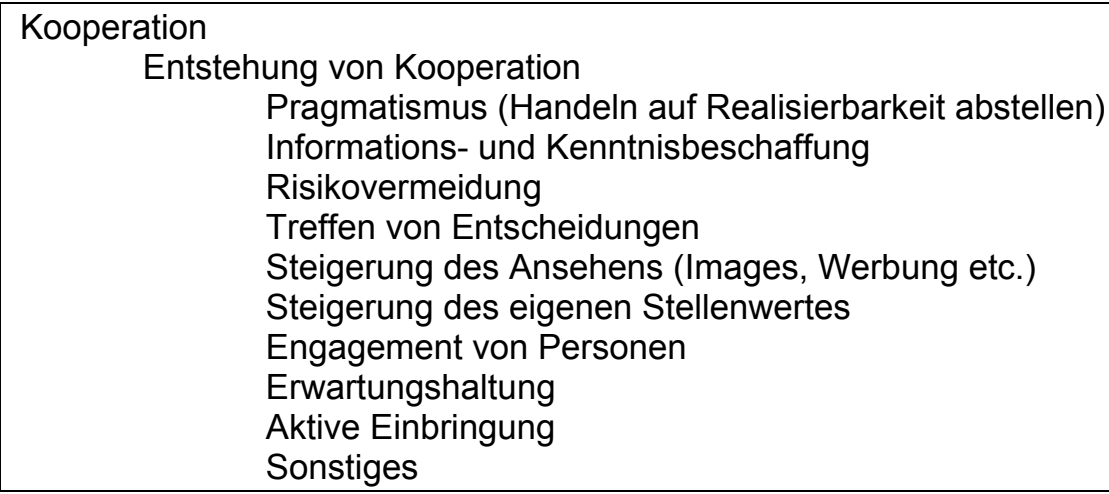

Abbildung 11: Entstehung von Kooperation

Die drei letztgenannten Codes der Kategorie Kooperation bzw. Entstehung von Kooperation (Codes 1. Ebene) ergeben sich aus dem Pre-Test der Studie und sind aufgrund der dort getätigten Aussagen und aufgedeckten Befunde in das Auswertungsschema aufgenommen worden. Die Steigerung der eigenen Position lässt sich, wie oben schon angedeutet, auf die Person beziehen, die sich in einer Institution mit der Kooperation beschäftigt und durch das kooperative Verhalten die eigene Stellung innerhalb der Institution festigen oder verbessern 
will. Das Engagement von Personen ist ebenfalls als Grund für die Entstehung von Kooperationsbeziehungen zu sehen, denn nur wenn Akteure an Schlüsselpositionen die Initiative zur Kooperation ergreifen, kann diese auch stattfinden. Die Erwartungshaltung, die an Institutionen herangetragen wird, kann ebenfalls einen Grund für die Entstehung von Kooperation darstellen. Diese Erwartungshaltung kann von übergeordneter Stelle angeordnet sein, z.B. politische Beschlüsse, die eine Kooperation unerlässlich machen um das vorgegebene Ziel zu erreichen. Der Code ,Aktive Einbringung' soll hervorheben, dass Institutionen mit aktiver Initiative zur Problemlösung beitragen können und so Kooperation erst entsteht.

In der Diskussion um die Kooperation von Institutionen zur Verfolgung der eigenen und gemeinsamen Interessen und dem Beitrag zu einer konkreten Problemlösung in Konfliktsituationen, müssen die Interessen der Akteure berücksichtigt werden.

Um die Kooperation von Akteuren umfassend darzustellen, werden die in der Theorie verankerten Interessen der Akteure in das Auswertungsschema aufgenommen.

Kooperation

Interessen

Identisch

Komplementär

Konkurrierend

Antagonistisch

Abbildung 12: Interessen von Akteuren

Der ,Verhandlungsmodus' wird ebenfalls als wichtiger Faktor von Kooperationsbeziehungen in die Analyse des Datenmaterials einbezogen. Hierzu werden die drei Verhandlungsmodi in Codes der Kategorie Kooperation eingeordnet. Weiterhin ist davon auszugehen, dass die Kooperation für einen Großteil der Institutionen und gerade für die Forstverwaltungen eine Innovation darstellen. Aus diesem Grund sollen die Innovation und deren Merkmale als Codes der Kategorie Kooperation das Analyseschema erweitern.

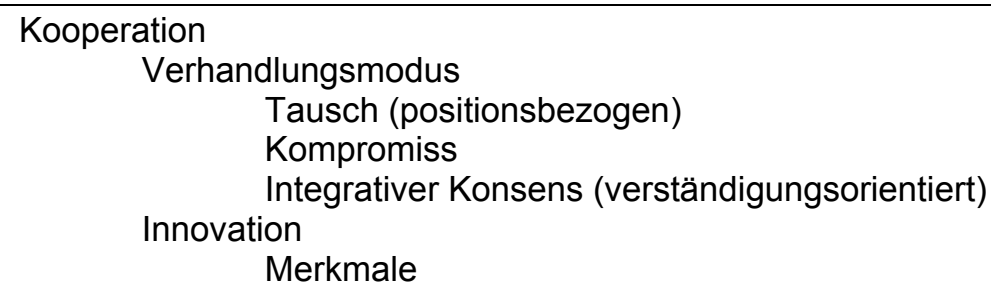

Abbildung 13: Verhandlungsmodus und Innovation

Neben den Innovationsmerkmalen, die sich durch Kooperation der unterschiedlichen Institutionen ergeben, interessieren weiterhin die Merkmale, die sich aus einer erfolgreichen integrativen Maßnahme ergeben. Die Kooperation in unterschiedlichen Projekten zur Integration kann Merkmale hervorbringen, die als Faktoren für die erfolgreiche Durchführung 
solcher Projekte gelten können. Durch diese Analysekategorie soll überprüft werden, ob die Institutionen in der Lage sind, Merkmale, die den Erfolg der Kooperationsprojekte beschreiben, herauszufiltern. Dies bezieht sich vor allem auf die Forstverwaltung, da die Hypothese eine geringe Beteiligung dieser an den Projekten vermutet. Aus den Merkmalen lassen sich anschließend Handlungsempfehlungen für die Zielsetzung in zukünftigen Kooperationsprojekten ableiten.

Die Kooperation sollte für eine Institution im günstigsten Fall mit einem Nutzen verbunden sein. Ein wichtiger Faktor, der nicht unberücksichtigt bleiben darf. Es ist davon auszugehen, dass der Forstsektor Integration von sozialen Problemgruppen nur in Kooperation mit anderen Institutionen leisten kann. In der Analyse soll vor allem der Nutzen abgebildet werden, der durch die Kooperation für die Forstverwaltungen entsteht.

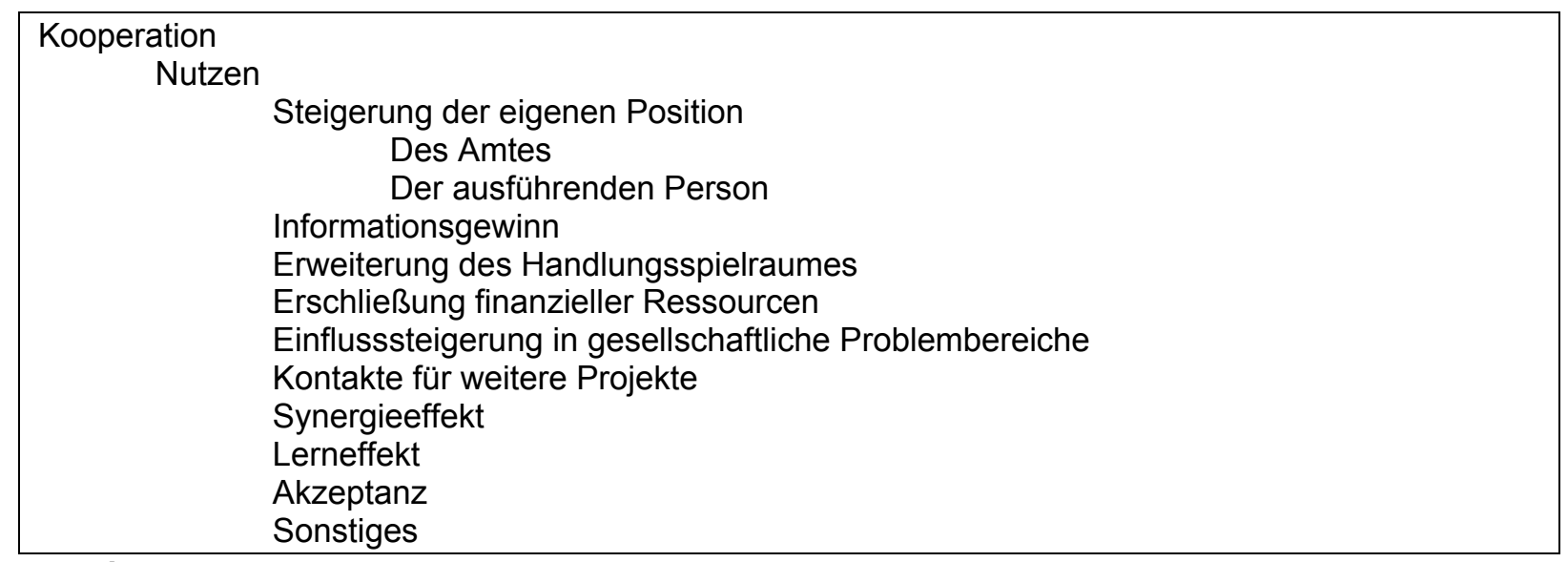

Abbildung 14: Nutzen der Kooperation 


\subsubsection{Hypothese III - Neues Produkt}

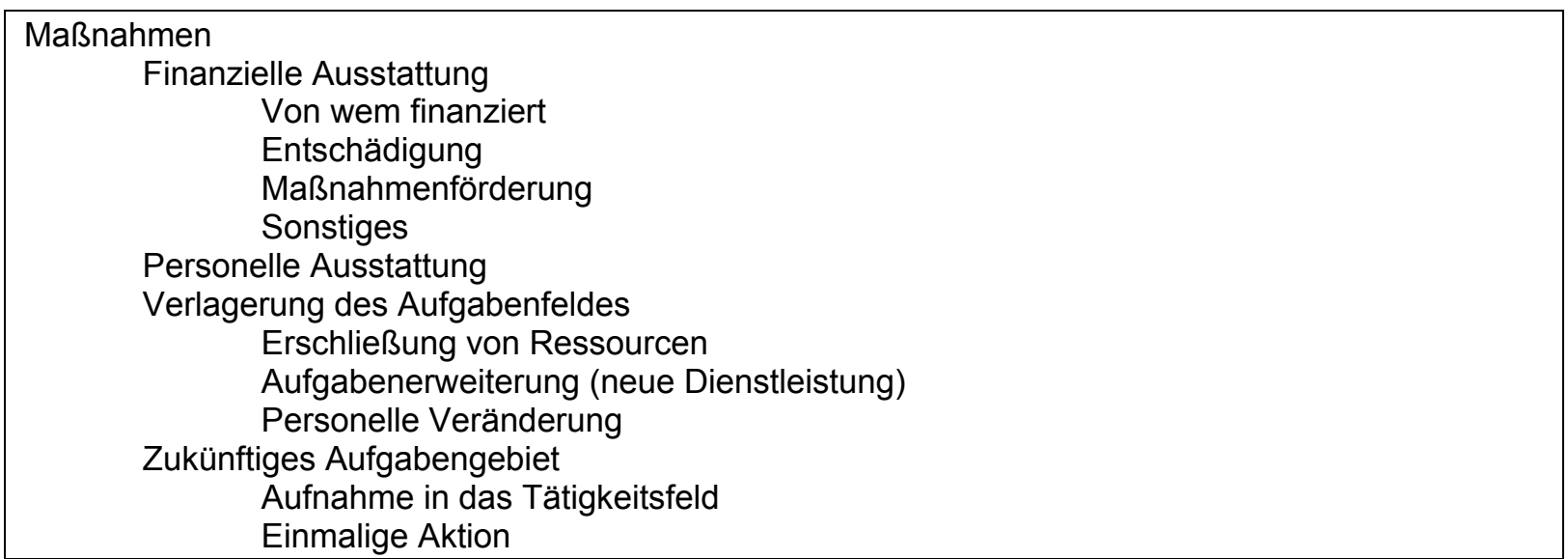

Abbildung 15: Ausstattung der Maßnahmen und Aufgabenerweiterung

Die Kategorie Maßnamen, die hier durch Codes unterschiedlicher Ebenen spezifiziert wird, soll zunächst Auskunft über die finanzielle und personelle Ausstattung der Integrationsprojekte geben. Diese Faktoren geben Auskunft über den Aufwand, den die Institutionen für diese Projekte betreiben. Der Code ,Verlagerung des Aufgabenfeldes' bezieht sich vor allem auf den Forstsektor, denn wie aus den o.g. Ausführungen hervorgeht, muss sich dieser mit der Entwicklung neuer Produkte auseinandersetzen. Es soll hier gezeigt werden, ob mit Integrationsprojekten Ressourcen für die Forstverwaltung erschlossen werden können, hieraus eine neue Dienstleistung entstehen kann und dies wiederum zu personellen Veränderungen innerhalb der Verwaltung führt. Der Faktor ,Zukünftiges Aufgabengebiet' soll verdeutlichen, ob die Maßnahmen zur Integration einen nachhaltigen Effekt auf die Aufgabenerfüllung der Forstverwaltung haben oder ob es sich nur um ein einmaliges Projekt handelt.

Nach der Darstellung des Kategoriensystems sind die Auswertungsmethoden, die sich dieser Grundlage bedienen, von Bedeutung.

\subsection{Auswertungsmethoden}

Im folgenden Kapitel sollen zunächst die allgemeinen Auswertungsmethoden der qualitativen Sozialforschung dargestellt werden. Daraufhin wird eine Präzisierung der dieser Arbeit zugrundeliegenden Methoden vorgenommen. 


\subsubsection{Qualitative Datenanalyse}

Für die Auswertung von qualitativen Interviews gelten vier allgemeine Phasen, die generelle Handlungsanweisungen und Strukturen geben und ebenso fallspezifische Modifikationen im weiteren Verlauf der Analyse zulassen. Die vier Phasen sollen im Einzelnen nachfolgend beschrieben werden:

1. Transkription: Die in den Interviews entstanden Gesprächsaufzeichnungen (im konkreten Fall liegen die Daten als audio-files vor) müssen verschriftlicht werden. Dieser Schritt erfordert eine große Konzentration und ist sehr zeitintensiv, da es sich um Alltagsgespräche handelt und der Gesprächsinhalt situations- und inhaltsgetreu wiedergegeben werden muss. Bei der schriftlichen Wiedergabe müssen Regeln für die nonverbalen Aspekte des Interviews festgelegt werden. So müssen Gesprächspausen, Lachen, Unterbrechungen etc. nach einem bestimmten Muster in den Text eingebunden werden und so interpretierbar sein. Ein wiederholtes Abhören des Gespräches soll entstandene Tipp- und Hörfehler ausmerzen. Bei der Transkription sollte direkt darauf geachtet werden, dass die genannten Personennamen anonymisiert und notwendige Daten (z.B. biographische Daten oder Statistiken), die nicht aus dem Material hervorgehen, aufgenommen werden (Lamnek, 2005). Die oben angesprochene Genauigkeit der Transkription ist für die folgende Analyse von großer Bedeutung. Denn werden Mimik und Gestik in dem Transkript vernachlässigt, kann die Interpretation in eine falsche Richtung laufen bzw. einige Passagen verlieren an Wichtigkeit (Knoblauch, 2006). Meuser und Nagel (2002) halten hingegen die genaue Aufnahme von Pausen, Stimmlagen oder sonstigen nonverbalen Aspekten für überflüssig, da es sich um "gemeinsam geteiltes Wissen“ (ebd., S. 83) zwischen Forscher und Experte handelt. Hier wird eher die Vollständigkeit der Transkription als kritisch erachtet. Die wortgenaue Abschrift des Interviews hängt von dem Diskursverlauf ab und davon, ob es sich um Betriebswissen (Experte als Zielgruppe) oder Kontextwissen (Experte als „Größe“, die im Zusammenhang mit der Zielgruppe steht) handelt. Ist das Gespräch unter idealen Bedingungen verlaufen und als gelungen zu bezeichnen (s. Kap. 4.1.2), bedarf es einer ausführlichen Transkription, weil dort sehr viel relevante Informationen enthalten sind. Ist das Gespräch nicht ideal verlaufen (Gründe Kap. 4.1.2) und als misslungen anzusehen, kann sehr selektiv transkribiert werden und der Fokus auf relevante Passagen gelegt werden. Gehört der Experte zur Zielgruppe der Untersuchung und berichtet über die Handlungsstrukturen, auf die er/sie direkt Einfluss nehmen kann, fällt auch hier die Transkription ausführlicher aus als bei Gesprächen mit Experten, die Auskunft über die Zielgruppen geben (ebd., S. 83). Da es sich bei den Experten um die Zielgruppe der Untersuchung handelt und deren Handlungs- und Entscheidungsstrukturen aufgedeckt werden sollen, wurde die Interviews fast ausschließlich wortgenau transkribiert. Von einigen Interviews, bei denen sich die „Kontextstellung“ der Gesprächspartner erst im Verlauf des Gespräches ergeben hat, wurden detaillierte 
Auflistungen der Gesprächsinhalte festgehalten, aber keine wortgenauen Abschriften angefertigt.

2. Einzelanalyse: Diese Analysephase ist von der Konzentration des auszuwertenden Materials geprägt. Die transkribierten Interviews werden einzeln einer Aufteilung in Nebensächlichkeiten und zentrale Passage unterzogen. Der neuentstandene, konzentrierte Text geht in eine Inhaltsanalyse ein, es werden Besonderheiten des Interviews bestimmt und schon erste allgemeingültige Schlüsse gezogen. Als Ergebnis dieser Phase sollte eine Beurteilung des einzelnen Interviews stehen, die Besonderheiten und das Allgemeine einbeziehen (Lamnek, 2005, S. 403f.). Bei Meuser und Nagel (2002, S. 84) besteht der erste Schritt nach der Transkription aus der Paraphrasierung des Experteninterviews. Hierbei handelt es sich um eine Verdichtung des Textes, der den Verlauf des Diskurses in den Worten des Forschers wiedergibt. Bei der Paraphrasierung ist darauf zu achten, dass dem Text inhaltlich nichts hinzugefügt wird, keine Information unterschlagen oder verzerrt wiedergegeben wird. Nach dieser Paraphrasierungsphase schließt sich bei Meuser und Nagel (2002) im speziellen Fall der Experteninterviews die Formulierung von Überschriften an. Der Text liegt nun in Sequenzen vor, die eine neue Verdichtung durch die Bildung von Überschriften erfahren. Diese sollte möglichst textnah und der Terminologie des Experten entsprechend gewählt werden. Im Falle des Experteninterviews kann hierdurch eine „Umordnung“ des Textes erfolgen, die in der klassischen Einzelanalyse nicht erlaubt ist.

3. Generalisierende Analyse: In dieser Phase wird nicht mehr nur ein Interview betrachtet, sondern alle Interviews auf deren Gemeinsamkeiten überprüft. Grundsätzliches Ziel dieses Schrittes ist die Gewinnung von allgemeinen Erkenntnissen, die theoretisch fundiert sind. Es geht hierbei nicht um eine Gleichmachung der Interviews, sondern darum, die Verschiedenartigkeit der Interviews herauszustellen. Inhaltliche Gemeinsamkeiten und Unterschiede können als Tendenzen für einige oder alle Befragten herausgestellt werden und unter Bezugnahme auf den Einzelfall interpretiert werden (Lamnek, 2005, S. 404). Auch Meuser und Nagel (2002, S. 87f.) beziehen sich nach der Sequenzierung der einzelnen Texte durch die Bildung von Überschriften auf alle Experteninterviews. Diese Phase wird als thematischer Vergleich bezeichnet, wobei Passagen aus allen Interviews, die gleiche oder ähnliche Themen behandeln, zusammengestellt und deren Überschriften vereinheitlicht werden. Diese Verdichtung des Materials sollte einer ständigen Kontrolle unterzogen werden, deren Basis der Gesamttext der Interviews darstellt. Im Anschluss an den thematischen Vergleich, werden die dem Text entnommenen Begriffe und Überschriften kategorisiert. Diese Kategorien entsprechen den soziologischen Übersetzungen der Textbausteine, z.B. die Formel „der Laden muss laufen“ wird übersetzt in „Primat der Funktionsfähigkeit der Verwaltung" (Meuser und Nagel, S. 88) und weist somit auf ein soziologisches Konzept der Verwaltung hin. Diese Abstraktionsebene der soziologischen 
Konzeptionalisierung entspricht einer empirischen Generalisierung, denn hierbei werden Aussagen über das Expertenwissen getroffen und auf dieser Grundlage die Geltung von soziologischen Konzepten überprüft (ebd., S. 89). Im nächsten Schritt der Analyse erfolgen eine Ablösung von den Interviews und die systematische Neuordnung der Kategorien. Mit dieser Neuordnung geht die Interpretation von empirisch generalisierten „Tatbeständen“ einher. Die Verknüpfung der Kategorien mit der Theorie und die theoriegestützten Interpretationen stehen bei diesem Schritt im Vordergrund. Die empirisch erhobenen Daten werden also theoretisch überprüft, fundiert und allgemeingültig interpretiert (ebd., S. 89ff.).

4. Kontrollphase: Im Analyseverlauf hat sich das Material stetig verringert und unterliegt so der Gefahr von Fehlinterpretationen. Um diesen Fehler zu umgehen, sollte immer wieder der Gesamttext des Interviews zur Analyse herangezogen werden. Die Informationsbasis kann in zweifelhaften Interpretationsfällen auch wieder erweitert werden, d.h. dem reduzierten Textfragment wird erneut Informationsmaterial aus den Interviews zugefügt. Für Untersuchungen, die in einem Forschungsteam durchgeführt werden, empfiehlt es sich die Ergebnisse auszutauschen und zu diskutieren und ggf. zu modifizieren (Lamnek, 2005, S. 404). Auch Meuser und Nagel (2002, S. 90f.) sehen in der Kontrolle und dem Rückbezug auf die vorhergegangenen Analyseschritte eine Notwendigkeit, um die Verallgemeinerung der Daten und Interpretationen aus den Experteninterviews zu gewährleisten. Besonders wichtig ist die Kontrolle des Verhältnisses von Theorie und Daten im gesamten Analyseprozess.

In der vorliegenden Arbeit wurden die Experteninterviews in der nach Meuser und Nagel (2002) definierten Methode erhoben und analysiert. Hierbei fand die Analyse nach den Kategorien statt, die aus der Theorie abgeleitet wurden und z.T. durch die Interpretation der Textbausteine um neue Kategorien erweitert wurden. Die Interviews wurden auf Grundlage der qualitativen Inhaltsanalyse ausgewertet, die Gegenstand des folgenden Kapitels ist.

\subsubsection{Qualitative Inhaltsanalyse}

Die Inhaltsanalyse bezieht sich zunächst generell auf jede Art von symbolischem Material, wozu vor allem Texte, Bilder, Tondokumente etc. zählen (Meuser, 2006). Hierbei steht die Inhaltsanalyse von Texten in der Sozialforschung im Vordergrund. Die zuvor nur in quantitativen Verfahren eingesetzte Inhaltsanalyse stieß auf Kritik, da sie die Sinngehalte der Texte vernachlässigte. Das von Mayring (1997) entwickelte Verfahren der qualitativen Inhaltsanalyse gehört zu den derzeit bekanntesten und häufig angewandten Verfahren. Es liegt dieser Arbeit als Methode zu Grunde und soll im Folgenden beschrieben werden.

Die qualitative Inhaltsanalyse hat vor allem die Analyse von Kommunikation zum Ziel, wobei die nachfolgenden Punkte zusammenfassend als generelle Ziele angesehen werden: 
"- Kommunikation analysieren;

- fixierte Kommunikation analysieren;

- dabei systematisch vorgehen;

- das heißt regelgeleitet vorgehen;

- das heißt auch theoriegeleitet vorgehen;

- mit dem Ziel, Rückschlüsse auf bestimmte Aspekte der Kommunikation zu ziehen.“

(Mayring, 1997, S. 13)

Texte sind in der Regel Gegenstand dieser Methode, wie oben bereits erwähnt. In dieser Arbeit handelt es sich um die Experteninterviews der einzelnen Fallbeispiele. Diese Interviews liegen in einer protokollierten Form vor, im konkreten Fall als Word-Dokumente, die zu einem späteren Zeitpunkt in das Analyseprogramm überführt werden können. Die Inhaltsanalyse soll einer Systematik folgen, die bestimmten Regeln unterliegt. Diese Regeln tragen zur Nachvollziehbarkeit und Überprüfung der Auswertung bei. Ein weiteres Kennzeichen der Systematik ist die theoriegeleitete Auswertung. Hierbei werden die Texte nach der Fragestellung analysiert, die aus der Theorie abgeleitet ist und die Ergebnisse vor dem Theoriehintergrund interpretiert. Jede Analysestufe nimmt hierbei Bezug auf die ausgewählte Theorie. Der letzte Punkt, den die qualitative Inhaltsanalyse verfolgt, ist der schlussfolgernde Aspekt. Die Analyse bleibt nicht auf der Textebene stehen, sondern stuft diesen als Teil des gesamten Kommunikationsprozesses ein. Die Rückschlüsse auf interessante Aspekte der Kommunikation können z.B. die Absichten des Befragten und die Wirkungen bei den Empfängern betreffen (ebd., S. 12). Der Teil der Theoriegeleitetheit spielt in der vorliegenden Arbeit eine wichtige Rolle. Die Fragestellungen wurden entsprechen aus der Theorie abgeleitet, das Analyseraster der Interviews wurde an die Theorie angelehnt und die Auswertung und Interpretation der Ergebnisse wurde vor dem Theoriehintergrund durchgeführt. Neben der Theorie- und Hypothesenbildung, der Pilot- oder Einzelfallstudie, der Vertiefung, Prozessanalyse und der Klassifizierung gehört die Theorie- und Hypothesenprüfung zu den Aufgaben der qualitativen Inhaltsanalyse. Letztere steht im Vordergrund für die vorliegende Untersuchung. Es ist demnach möglich, mit der Methode der qualitativen Inhaltsanalyse bestehende Theorien und Hypothesen zu überprüfen und ggf. umzustrukturieren. Lamnek (2005) unterscheidet zwei Formen der qualitativen Inhaltsanalyse. Eine Form der qualitativen Inhaltsanalyse unterscheidet sich von der quantitativen Innhaltsanalyse nur dadurch, dass sie nicht quantifiziert oder in Teilbereichen nicht quantifiziert (ebd., S. 506). Ferner werden bei der qualitativen Inhaltsanalyse theoretisch entwickelte Analyseeinheiten, -dimensionen und -kategorien auf die systematischen Dokumente angewendet. In diesem Punkt unterscheidet sich die zweite Form der qualitativen Inhaltsanalyse deutlich von der ersten Form. Bei dieser Analysemethode bestehen im Vorfeld keine theoretischen Analysekriterien (ebd.).

Das qualitative Merkmal der Inhaltsanalyse nach Mayring (1997) stellt die Offenheit für Kategorien dar, die empirisch aus dem Datenmaterial gewonnen werden. Das eher quantitativ zu bewertende Merkmal der Methode ist die Einzelfallbetrachtung, bei der die 
Kategorien den Fall analysieren und eine Sammlung von Merkmalsausprägungen darstellen (Lamnek, 2005, S. 518).

Die qualitative Inhaltsanalyse nach Mayring (1997) behält die Stärken der quantitativen Inhaltsanalyse bei und entwickelt vor deren Hintergrund Verfahren systematischer qualitativ orientierter Textanalyse. Zunächst sollen einige wichtige Voraussetzung bzw. Besonderheiten der Inhaltsanalyse herausgestellt werden. Der zu analysierende Text soll immer im Kommunikationszusammenhang gesehen werden und an diesen angelehnt, interpretiert werden. Die Analyse der Texte sollte nach einer festgelegten Systematik erfolgen. Die Systematik besteht in der Orientierung an im Vorfeld festgelegten Regeln der Textanalyse, wobei die Aufstellung eines Ablaufmodells für die Analyse einen zentralen Aspekt einnimmt. Es geht hierbei um die genaue Definition der Analyseschritte und deren Reihenfolge. Aufgestellte Regeln für die Analyse und die Interpretation haben vor allem die Nachvollziehbarkeit der Inhaltsanalyse und das erneute Durchführen der Analyse durch einen weiteren Auswerter zum Ziel (Mayring, 1997, S. 42f.). Als weitere wichtige Voraussetzung der qualitativen Inhaltsanalyse ist das Kategoriensystem zu bezeichnen. Diese Vorgehensweise wird von der quantitativen Analyse übernommen und stellt das zentrale Element der Analyse dar (ebd., S. 43). Das Kategoriensystem wird meist als Ergebnis der Analyse verstanden und trägt zur Vergleichbarkeit der Ergebnisse bei. Die Inhaltsanalyse in qualitativer Forschung soll immer an den Gegenstand der Untersuchung gebunden sein und auf diesen modifiziert werden. Um diesen Gegenstandsbezug herzustellen, stehen drei Grundverfahren der Analyse zur Verfügung: Zusammenfassung, Explikation und Strukturierung (ebd., S. 44). Ein weiterer wichtiger Punkt in der qualitativen Inhaltsanalyse ist die Theoriegeleitetheit. Dies bedeutet, dass in alle Verfahrensentscheidungen der Stand der Forschung systematisch einbezogen werden muss. Auch die Einbeziehung von quantitativen Analyseschritten ist ein wichtiges Merkmal der qualitativen Inhaltsanalyse. So können z.B. zur Verallgemeinerung von Ergebnissen die Häufigkeit der aufgetretenen Fälle hinzugezogen werden. Die Häufigkeit einer Kategorie innerhalb eines inhaltsanalytischen Kategoriensystems kann Auskunft über die Bedeutung der Kategorie geben (ebd., S. 45). Die Gütekriterien, Objektivität, Reliabilität (Zuverlässigkeit) und Validität (Gültigkeit) über die Einschätzung der Ergebnisse sollten in der qualitativen Inhaltsanalyse nicht vernachlässigt werden. Hauptaugenmerk liegt hierbei auf der sog. Interkoder - Reliabilität, welche die Ergebnisse von mehreren Inhaltsanalytikern vergleicht, die unabhängig voneinander das gleiche Material analysieren (ebd., S. 46). Sind die Voraussetzungen und Merkmale der qualitativen Inhaltsanalyse gegeben, lassen sich die einzelnen Analyseschritte und der Ablauf der Analyse festlegen. Die einzelnen Schritte sind in der folgenden Abbildung dargestellt und sollen im Einzelnen kurz erläutert werden. 


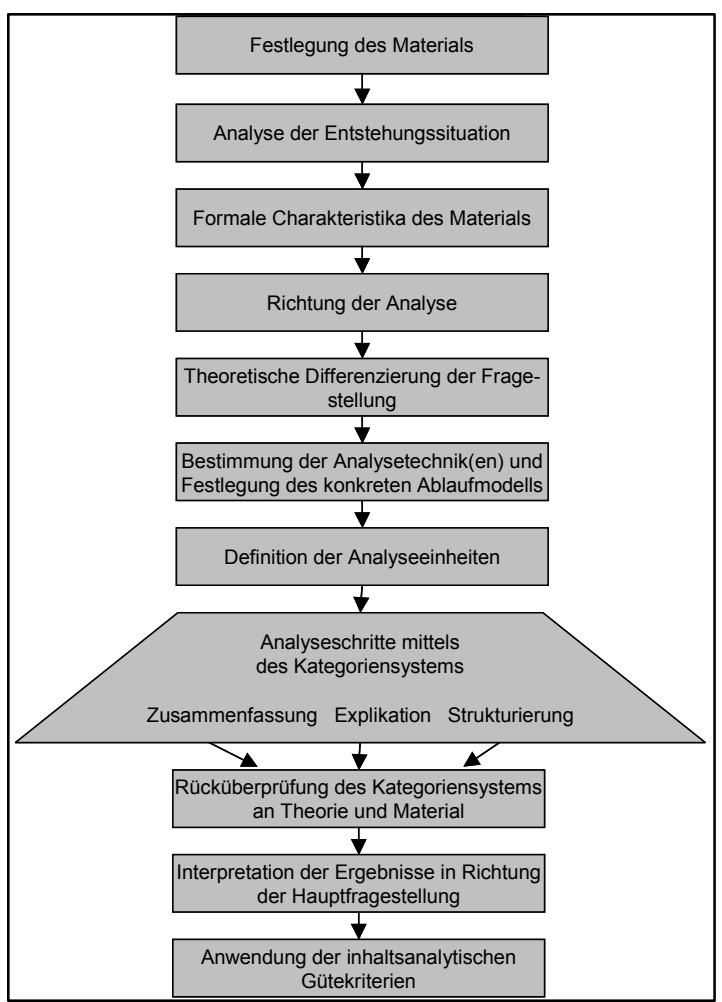

Abbildung 16: Allgemeines inhaltsanalytisches Ablaufmodell (Mayring, 1997, S. 54)

Als erste Stufe im Ablauf der Analyse ist die Festlegung des Materials zu sehen. Hier geht es um die genaue Definition des Materials, welches in die Analyse einbezogen werden soll. Dieser Schritt kann mit der Stichprobenziehung verglichen werden, denn es müssen Überlegungen über Definition der Grundgesamtheit, auf die sich die Aussagen beziehen sollen, über den Stichprobenumfang, der nach den Kriterien der Repräsentativität und Forschungsökonomie gewählt sein sollte und über das Modell der Stichprobenziehung (Zufallsauswahl, Auswahl nach festgelegten Quoten etc.) angestellt werden (ebd., S. 47). In dem konkreten Fall der vorliegenden Untersuchung handelt es sich um eine Grundgesamtheit von 27 Interviews, die mit Vertretern einzelner Verwaltungen und Organisationen geführt wurden und Projekte zur Integration von sozialen Problem- bzw. Randgruppen mit Waldbezug zum Inhalt hatten. Die Größe der Stichprobe hängt in erster Linie von den finanziellen und zeitlichen Aspekten des Forschungsvorhabens ab. Die Auswahl der Interviewpartner verlief durch das Vorhandensein sozialintegrativer Projekte, was in einem ersten Telefonat zur Kontaktaufnahme aufgedeckt wurde. Zudem fanden auch Verweise einzelner Interviewpartner auf weitere Gesprächspartner statt, die an den konkreten oder ähnlichen Projekten beteiligt waren. Die Studie kann nur in Teilen für den Forstbereich als repräsentativ gelten, was auf die Häufigkeit der auftretenden Fälle zurückzuführen ist. Nach der Festlegung des Materials steht als zweiter Schritt die Analyse der Entstehungssituation an. Diese beinhaltet genaue Angaben, von wem und unter welchen 
Bedingungen das Material produziert wurde. Hierzu gehören Kriterien wie, Informationen zum/r Verfasser/in des Materials, der Handlungshintergrund (emotional und kognitiv) des/der Verfassers/in und, die Zielgruppe, die konkrete Erhebungssituation etc. (ebd.). Im konkreten Fall bedeutet dies, dass die Teilnahme an den Interviews freiwillig war. Ein gegenseitiges Interesse an der Untersuchung wurde durch die direkte Bereitschaft zur Teilnahme an der Befragung, durch die positive Bewertung des Interesses an der eigenen Organisation der Befragten und an z.T. vorbereitetem Material für das Interview und die Herausgabe dessen deutlich. Bei den Gesprächen handelt es sich um teilstrukturierte und offene Interviews (s.o. Kap. 4.1.2). Die Interviews wurden von der Verfasserin persönlich durchgeführt und fanden hauptsächlich in den Institutionen und Organisation, demnach den Arbeitsplätzen der Gesprächspartner statt. In drei Fälle wurde das Interview nicht in den Räumen der Institution bzw. Organisation der Befragten geführt, sondern in einem Fall bei dem Gesprächspartner zu Hause aus familiären Gründen, in einem anderen Fall in einem Café, da zum Gespräch die Mittagspause genutzt wurde und in einem weiteren Fall fand das Gespräch im Wald statt, da es sich hierbei um ein Arbeitsprojekt handelt und so konkrete Eindrücke und Erfahrungen aufgenommen werden konnten. Bei diesem Gespräch konnte nur ein Gedächtnisprotokoll im Anschluss an das Gespräch notiert werden, da eine Aufzeichnung unter den Umständen eines ständig wechselnden Standortes nicht möglich war. Als dritter Punkt des Analysemodells sind die formalen Charakteristika des Materials festzulegen. Hierbei ist die Form des Materials von großer Bedeutung, was meist als niedergeschriebener Text vorliegen sollte. Beobachtungen und weitere zusätzliche Informationen können in den Text aufgenommen werden. Für den Transkriptionsvorgang sollten im Vorfeld Protokollierungsregeln festgelegt sein (ebd.). In dieser Untersuchung wurden die Gespräche mit Hilfe eines digitalen Aufnahmegerätes aufgezeichnet und im Anschluss von der Verfasserin transkribiert, so dass alle Interviews in Word-Dateien vorliegen. Die Transkription folgte hierbei folgenden Regeln:

- die Interviews wurden zu Beginn stichpunktartig, aber wörtlich transkribiert

- Laufe des Forschungsprozesses (nach der Explorationsphase bzw. Pre-Test-Phase) wurde zu einer vollständigen und wörtlichen Transkription übergegangen, da die stichpunktartigen Transkripte ebenso umfangreich waren, wie die vollständig transkribierten Interviews und auch so jegliche Information verarbeitet werden kann;

- Dialekte wurden ins Hochdeutsche übersetzt, nur bei echten Dialektausdrücken wurden die Begriffe nach dem Gehör übernommen;

- Füllwörter wie „äh“ oder „ehm“ wurden nicht aufgenommen;

- längere Pausen wurden durch Punkte, wie z.B. ....., verdeutlicht;

- Auffälligkeiten im Sprachverlauf wurden in Klammern aufgenommen (z.B. lacht); 
- Wörter, die auf der Aufzeichnung nicht zu verstehen sind, wurden wie folgt gekennzeichnet: .... (nicht zu verstehen, Verf.);

- Interviewer und Befragte wurden durch Kürzel vor Beginn der Rede- bzw. Textsequenz gekennzeichnet.

Nach Festlegung der Formalia des Materials beschäftigt sich die nächste Stufe des Analysemodells mit der Richtung der Analyse. Diese bestimmt, nach welchen Kriterien, Richtungen die Analyse stattfinden soll und Aussagen aus dem Text herausinterpretiert werden sollen. Zum einen kann der im Text behandelte Gegenstand beschrieben werden, es können Informationen über den Textverfasser herausgearbeitet oder die Wirkungen des Textes bei der Zielgruppe untersucht werden (ebd., S. 50). In der vorliegenden Arbeit sollen durch die Interviews Informationen zu sozial-integrativen Projekten, zur Kooperation in den Projekten zwischen den einzelnen Institutionen und dem forstlichen Nutzen solcher Projekte gewonnen werden. Das es sich um Experteninterviews handelt, ist die Richtung der Analyse auf den kognitiven Hintergrund und den Handlungshintergrund des Kommunikators ausgelegt. Der kognitive Hintergrund der Befragten spiegelt den Wissenshintergrund, die Erwartungen, Interessen und Einstellungen (ebd., S. 51) der Experten wider. Der Handlungshintergrund soll Aufschluss über die Intentionen, Pläne, Machtressourcen und bisherigen Handlungen (ebd., S. 51) der Interviewpartner geben. Nachdem die Richtung der Analyse festgelegt ist, wird die Fragestellung einer theoriegeleiteten Differenzierung unterzogen. D.h., die Theoriegeleitetheit der Analyse, die ein wichtiges Merkmal der qualitativen Inhaltsanalyse darstellt, basiert auf einer theoretisch begründeten inhaltlichen Fragestellung (ebd., S. 52). Für die vorliegende Arbeit werden aus den Theorien der „Sozialen Integration“ nach Fuchs (1999) und Esser (2001) und den kooperationstheoretischen Überlegungen (Benz, 1994) die Fragestellungen bzw. Hypothesen formuliert (s. Kap. 3.2.1 u. 3.2.2). Dieser Schritt der Analyse wird im konkreten Fall mit der Hypothesengenerierung gleichgesetzt. Nach der theoretischen Differenzierung der Fragestellung geht es im nächsten Schritt um die Festlegung der Analysetechniken und die Aufstellung eines Ablaufmodells. Das Ablaufmodell zerlegt die Analyse in einzelne Interpretationsschritte, die festgelegt werden müssen. Ziel hierbei ist es, die Analyse für Dritte nachvollziehbar und überprüfbar zu machen. Das Modell sollte auf das jeweilige Material und die Fragestellung ausgerichtet sein (ebd., S. 53). Das in der Abbildung 16 dargestellte Modell des Ablaufschemas besitzt allgemeinen Charakter und liegt dieser Arbeit zugrunde. Die Festlegung der Analysetechnik, die in diesem Schritt zusätzlich beachtet wird, wird in drei Grundformen des Interpretierens von Texten unterteilt:

„Zusammenfassung:

Ziel der Analyse ist es, das Material so zu reduzieren, daß die wesentlichen Inhalte erhalten bleiben, durch Abstraktion einen überschaubaren Corpus zu schaffen, der immer noch Abbild des Grundmaterials ist.

Explikation: 
Ziel der Analyse ist es, zu einzelnen fraglichen Textteilen (Begriffen, Sätzen,...) zusätzliches Material heranzutragen, das das Verständnis erweitert, das die Textsstelle erläutert, erklärt, ausdeutet.

Strukturierung:

Ziel der Analyse ist es, bestimmte Aspekte aus dem Material herauszufiltern, unter vorher festgelegten Ordnungskriterien einen Querschnitt durch das Material zu legen oder das Material aufgrund bestimmter Kriterien einzuschätzen." (Mayring, 1997, S. 58)

Die in der Arbeit angewandte Interpretationsform ist die strukturierende Inhaltsanalyse. Allgemeines Ziel der Strukturierung ist, die Textbestandteile, die Kategorien zugeordnet werden konnten, aus dem Material zu extrahieren. Die Strukturierung des Textmaterials kann auf vier Ebenen stattfinden: formale, inhaltliche, typisierende und skalierende Strukturierung. Die formale Strukturierung stellt die innere Struktur des Materials nach festgelegten, formalen Strukturierungsaspekten fest. Die typisierende Strukturierung filtert aus dem Material einzelne markante Ausprägungen und beschreibt diese. Eine Einschätzung des Materials nach Dimensionen und Skalenpunkten ist Ziel der skalierenden Strukturierung. Die inhaltliche Strukturierung fasst das Material nach bestimmten Themen zusammen und extrahiert diese aus dem Gesamtmaterial (ebd., S. 85). Diese spezifische Form der Interpretation bzw. Analyse wurde in der vorliegenden Arbeit genutzt. Die Filterung bestimmter Themen, Inhalte oder Aspekte mit Hilfe der inhaltlichen Strukturierung aus dem Interviewmaterial, wird durch das theoriegeleitete Kategoriensystem bzw. die theorieabhängigen Kategorien festgelegt (ebd., S. 89). So wurde auch in dieser Untersuchung jedes einzelne Interview durch das aus den Theorien entwickelte Kategoriensystem strukturiert, indem die interessierenden Aspekte den Kategorien zugeteilt und so aus dem Gesamttext extrahiert wurden. Sind Analysetechnik und Ablaufmodell bestimmt, sollten die Analyseeinheiten festgelegt werden. Diese können wiederum in drei Einheiten aufgeteilt werden: Kodiereinheit, Kontexteinheit und Auswertungseinheit.

„Die Kodiereinheit legt fest, welches der kleinste Materialbestandteil ist, der ausgewertet werden darf, was der minimale Textteil ist, der unter eine Kategorie fallen kann.

Die Kontexteinheit legt den größten Textbestandteil fest, der unter eine Kategorie fallen kann.

Die Auswertungseinheit legt fest, welche Textteile jeweils nacheinander ausgewertet werden." (Mayring, 1997, S. 53)

Als kleinste Texteinheit, die einer Kategorie zugeordnet werden kann, wurden in der vorliegenden Arbeit einzelne Wörter definiert. Diese müssen nachvollziehbar bzw. den eigentlichen Satzinhalt wiedergebend in die Kategorien eingeordnet werden, so dass in den meisten Fällen wenigstens zwei Wörter bzw. ganze Sätze als kleinste Einheiten kategorisiert wurden. Die Kontexteinheiten wurden im konkreten Beispiel als ganze Gesprächsabschnitte festgelegt. So finden sich z.T. in den Kategorien thematisch zusammenhängende Absätze aus den Interviews wieder. Die Auswertungseinheit der vorliegenden Untersuchung stellen die einzelnen transkribierten Interviews dar. Die Auswertung hat in der Reihenfolge der Erhebungszeitpunkte stattgefunden. Sind diese Stufen im Analyseprozess abgehandelt bzw. 
definiert worden, kann in die eigentliche Analyse der Daten beginnen. Wie oben schon erwähnt, wird die Inhaltsanalyse nach drei Grundformen vorgenommen. In der strukturierenden Inhaltsanalyse wird das Textmaterial in einem ersten Schritt mit Hilfe des Kategoriensystems analysiert. Ziel in diesem Schritt ist die Herausstellung von interessanten Aspekten und die erste Interpretation der Daten. An diesen ersten Analyseschritt schließt sich die Stufe der Rücküberprüfung des Kategoriensystems anhand der Theorie und des Materials an. Durch diesen wichtigen Schritt der Überprüfung hat der Forscher die Möglichkeit, das Kategorienschema zu erweitern oder zu kürzen. So kann der Fall eintreten, dass die Theorie eine Kategorie vorgibt, diese aber in den ersten Interviews so nicht aufgedeckt wird bzw. gar nicht auftritt. Diese Kategorie kann daraufhin modifiziert oder ganz aus dem Schema genommen werden. Die Besonderheit an der qualitativen Inhaltsanalyse ist die induktive Kategorienbildung. Durch die Analyse des Materials können im Verallgemeinerungsprozess Kategorien direkt aus den Gesprächen gebildet werden (Mayring, 1997, S. 74f.). Hierbei wird kein Bezug zu einer Theorie hergestellt. In dieser konkreten Untersuchung wurde das Hauptaugenmerk auf die Kategorienbildung aus den verschiedenen Theorien gelegt. Dennoch wurde das Kategoriensystem in den ersten Analyseschritten um Kategorien erweitert, die sich aus den Interviews als beachtenswert ergeben haben, z.B. durch Mehrfachnennung der Experten. Dieses Prozedere wurde gewählt, um eine möglichst genaue Abbildung der Realität und eine detailgetreue Interpretation zu gewährleisten. An die Modifizierung des Kategoriensystems schließt sich die Interpretation der Ergebnisse in Richtung der Hauptfragestellung an und die Anwendung der inhaltsanalytischen Gütekriterien. Die konkrete Interpretation der Ergebnisse findet sich im anschließenden Kapitel. Zur Anwendung kamen in dieser Untersuchung die Gütekriterien Re-Test-Reliabilität, wobei die Analyse ein weiteres Mal vorgenommen und geprüft wird, ob dieselben Ergebnisse erzielt werden (ebd., S. 109). Konkret wurde hier die Kategorisierung der Texte ein zweites Mal durchgeführt und ergab nur minimale (in 19 Fällen von 1077 Kodierungen) Neuzuordnungen bzw. Abweichungen zur ersten Analyse. D.h. die Genauigkeit und Zuverlässigkeit der Messung bzw. Analyse der Daten kann als gegeben angesehen werden. Die Gültigkeit der Datenanalyse soll unter Zuhilfenahme des Außenkriteriums und der Konstruktvalidität getestet werden. Um die Validität über das Außenkriterium festzustellen, werden Untersuchungsergebnisse, die einer ähnlichen Fragestellung nachgehen und einen entsprechenden Untersuchungsgegenstand zur Basis haben, als Vergleichsmaßstab herangezogen (ebd., S. 109). Die Konstruktvalidität setzt zur Plausibilitätsprüfung der Ergebnisse bewährte Theorien ein und prüft die operationalen Definitionen vor dem Theoriehintergrund (ebd., S.110). Die Feststellung der Konstruktvalidität wird in dem Ergebnisteil dieser Arbeit erörtert. Aussagen über die Interkoderreliabilität sind in der vorliegenden Arbeit nicht möglich, da allein die Verfasserin 
mit der Analyse der Interviews betraut war und aus forschungsökonomischen Gründen die Mehrfachanalyse durch mehrere Forscher nicht möglich war. Zur Analyse des Textmaterials können allgemein auch Computerprogramme hinzugezogen werden. In diesem Fall wurde das qualitative Datenanalyseprogramm MAXQDA verwendet. Die Nutzung des Programms soll Inhalt des folgenden Kapitels sein.

\subsubsection{MAX Qualitative Daten Analyse (MAXQDA)}

Bei dem Programm handelt es sich speziell um ein Datenbankprogramm (VERBI Software Consult. Sozialforschung $\mathrm{GmbH}$ ). Die Funktion des Programms besteht vornehmlich darin, Textstellen zu kodieren und diese dann in einer separaten Datenbank zu organisieren (Mayring, 1997, S. 101). In das Programm werden die Texte importiert, die als Rich Text Format-Dateien vorliegen müssen. Diese Texte bilden ein Projekt. Das konkrete Projekt ist in die Fallstudien unterteilt und enthält die Transkripte der geführten Interviews. Der Vorteil dieses Programms liegt in der Übersicht der gesamten Texte, s. Abb.18.

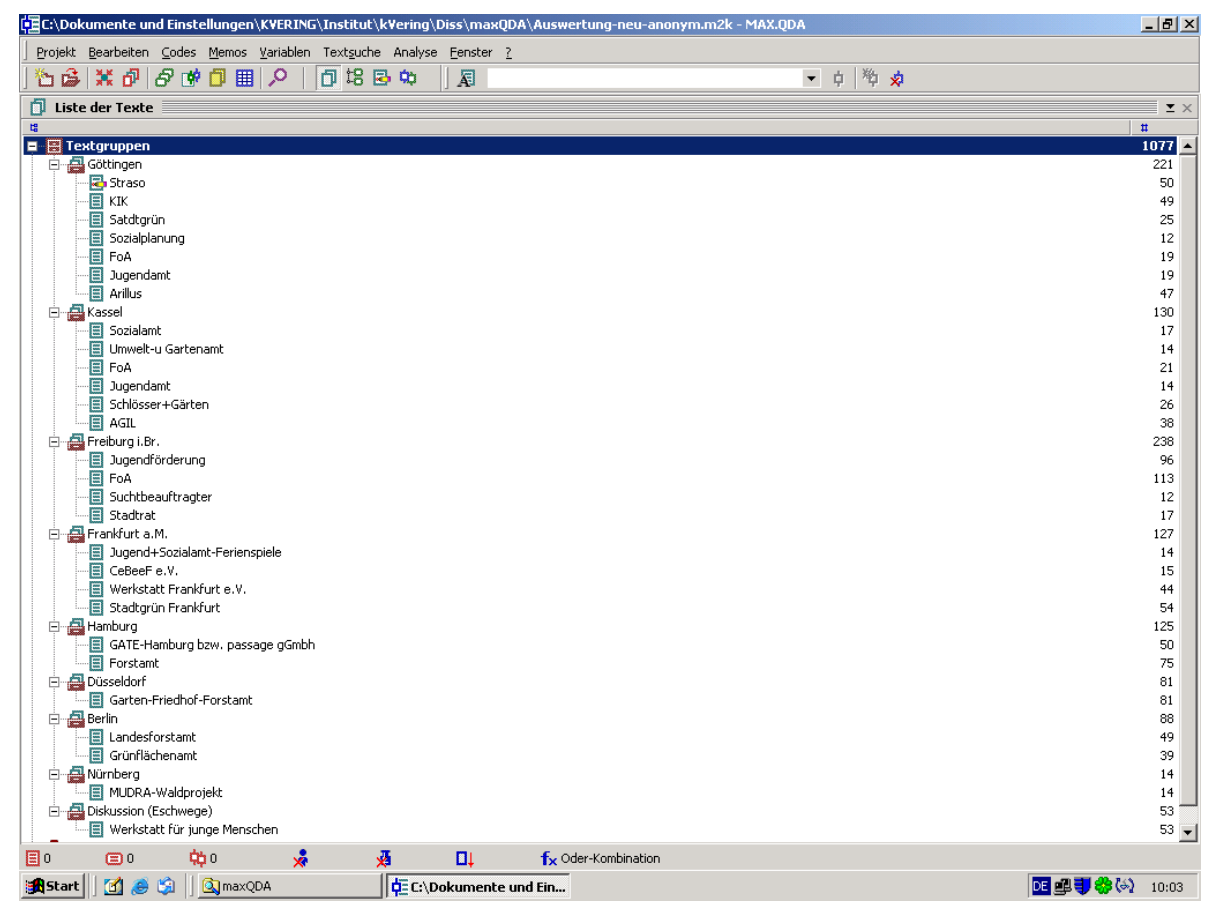

Abbildung 17: Liste der Texte (MAXQDA)

Neben der Darstellung der gesamten Texte kann in einem weiteren Übersichtsfenster jeweils ein Text angezeigt werden. Dieses Fenster bietet die Möglichkeit, Segmente des Textes zu markieren und generell zu editieren (Kuckartz, 2001). Ein weiteres Fenster, „Liste der Codes“, enthält das Kategoriensystem. Über die gemeinsame Anzeige der Fenster ist ein Gesamtüberblick möglich. Die Besonderheit hierbei besteht in der technisch einfachen 
Zuordnung der Textsegmente zu den einzelnen Kategorien. Mit Hilfe der „Drag and Drop“ Funktion können Textteile markiert und in die Kategorie eingefügt werden, der sie nach inhaltsanalytischen Aspekten angehören. Durch die Zuordnung der Textteile zu einer Kategorie werden die Textstellen mit der Kategorie sichtlich im Übersichtsfenster „Text“ gekennzeichnet.

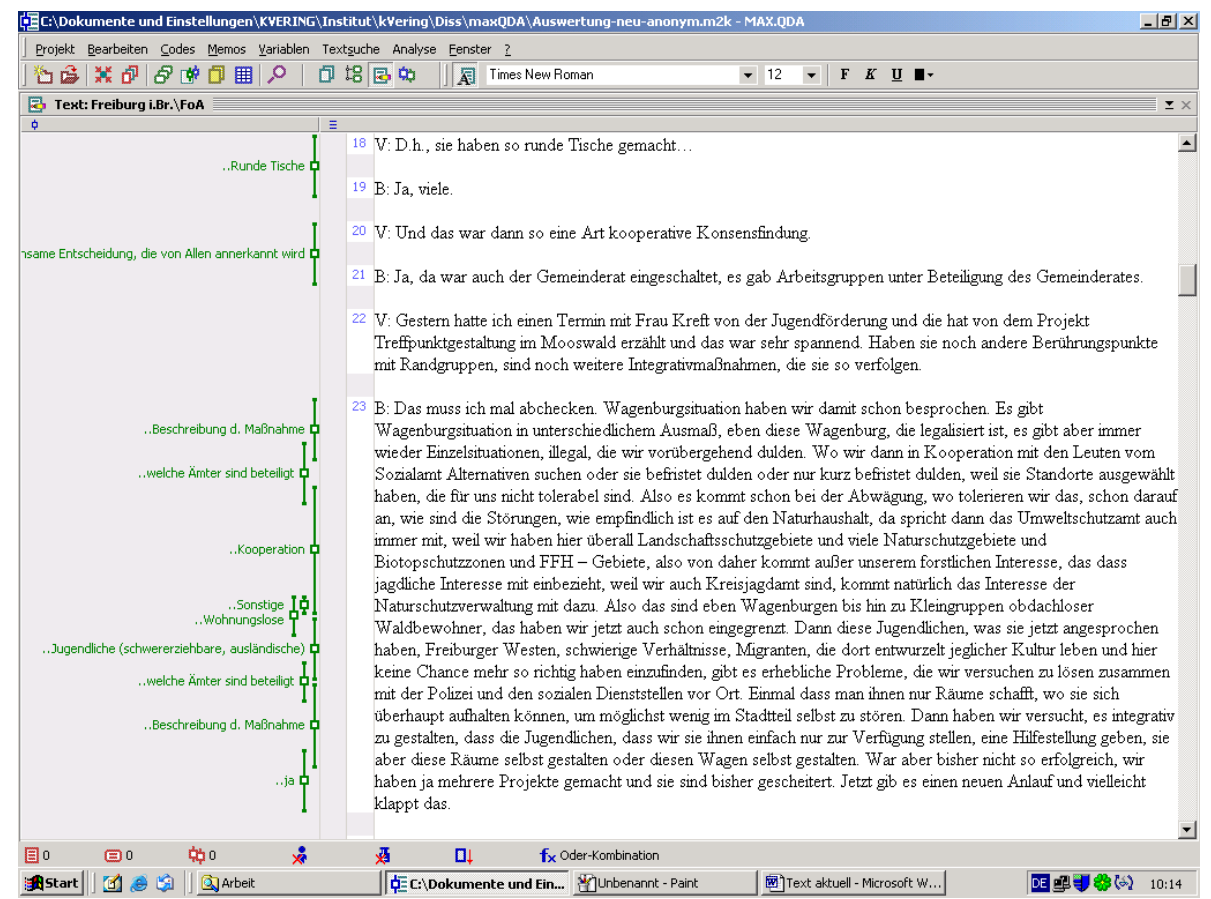

Abbildung 18: Beispiel für die Kategorisierung der Texte (MAXQDA)

Nach Analyse bzw. Kodierung aller relevanten Textstellen können einzelne Texte aus dem Projekt und einzelne Kategorien aktiviert werden. Die aktivierten und kodierten Textsegmente werden daraufhin in der "Liste der Codings“ angezeigt. Diese Funktion des Wiederfindens von zuvor kodierten Textstellen wird als Text-Retrieval bezeichnet (Kuckartz, 2001, S. 25). 


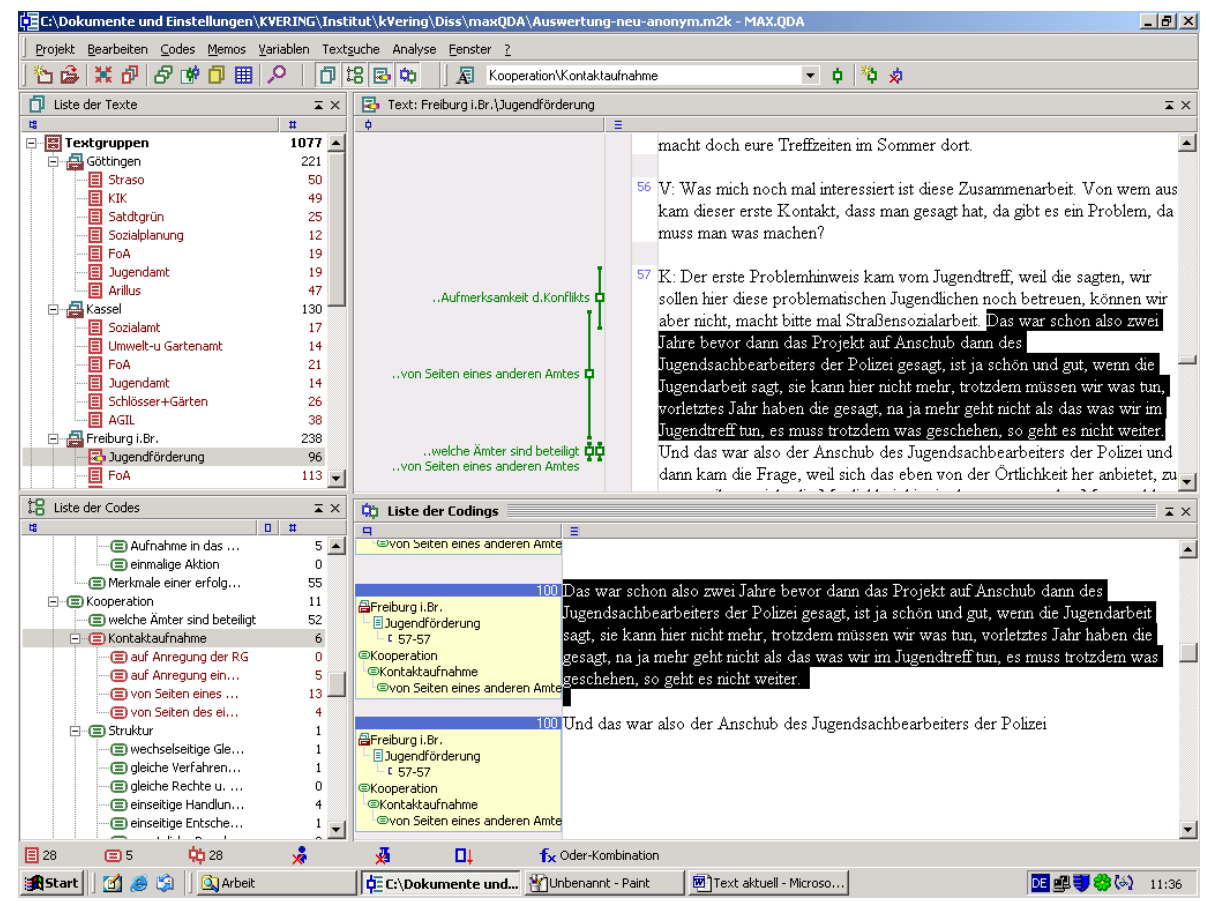

Abbildung 19: Gesamtdarstellung der Analyse (MAXQDA)

Das zentrale Kategoriensystem, als analytisches Instrument zur systematischen Datenauswertung weist vor allem drei hervorzuhebende Merkmale auf:

"- es kann ein hierarchisches System von Codes definiert werden

- die Zahl der Codes ist nicht begrenzt

- die Hierarchieebenen können bis zu zehn Stufen tief gestaffelt werden. (Kuckartz, 2001, S. 18)

In der vorliegenden Untersuchung wurden maximal vier Hierarchieebenen unterschieden, hier als "Code 1. Ebene“ bis "Code 4. Ebene“ bezeichnet. Besonders hingewiesen sei hier erneut sowohl auf die Kategorienherleitung aus den zugrundeliegenden Theorien als auch auf die induktive Kategorienbildung, welche die Möglichkeit bietet, zusätzlich Kategorien aus dem Datenmaterial zu bilden. 


\section{Ergebnisse und Ergebnisdiskussion}

Nach der Festlegung der Auswertungsmethoden sollen nun die Ergebnisse dargestellt, interpretiert und diskutiert werden.

\subsection{Deskription}

In diesem Kapitel sollen zunächst die beschreibenden Faktoren der Analyse dargestellt werden. Hierbei handelt es sich um die Berührungspunkte, welche die interviewten Institutionen mit den Problem- bzw. Randgruppen haben und welcher Art diese Kontakte sind. Weiterhin dient dies Kapitel der Eingrenzung und Charakterisierung der einzelnen Problemgruppen.

\subsubsection{Berührungspunkte der Institutionen mit Randgruppen}

Soll der Beitrag von Institutionen zur Integration von sozialen Problem- bzw. Randgruppen aufgezeigt werden, muss zunächst geklärt werden, ob überhaupt Berührungspunkte mit diesen Personengruppen bestehen und welcher Art diese Kontakte sind. In den häufigsten Fällen handelt es sich um Konflikte, die zwischen den Randgruppen und der übrigen Gesellschaft bestehen. Nur wenn Kontakt und ein Konflikt besteht, müssen Institutionen zu Problemlösungen in der Lage sein bzw. besteht erst die Notwendigkeit der Problemlösung. Die Berührungspunkte bzw. Kontakte mit den verschiedenen Problemgruppen werden von den unterschiedlichen Institutionen aufgrund ihrer Struktur ganz unterschiedlich beschrieben. So ist der Kontakt z.B. mit der Straßensozialarbeit in Göttingen zweigeteilt. Zum einen kommen die Menschen dort freiwillig hin, um z.B. ihre Post zu holen oder zu duschen und zum anderen werden sie für die Beschäftigungsprojekte der Einrichtung vom Sozial- oder Arbeitsamt geschickt (I1). Diese Art von Kontakt ist den Beschäftigungseinrichtungen gemeinsam. In den Interviews wird deutlich, dass die Berührungspunkte größtenteils einen Konflikt zum Anlass haben. Die Konflikte können unterschiedlicher Art sein, wobei die häufigste Nennung der „Aufenthalt an Orten“ (24 von 62 Nennungen; 38,7\%) als Konflikt beschrieben wird. Hierbei handelt es sich vornehmlich um den Aufenthalt von Problemgruppen auf Grünflächen und öffentlichen Plätzen:

„Ja, die Konflikte sind da mit den deutschen Anwohnern, die das so als Lebensgefühl nicht haben. Weil das Leben nach außen zu verlagern, das ist ja für deutsche Familien relativ ungewöhnlich." (I1) 
„...ca. 3000 Nutzer illegaler Drogen in Kassel, die im alläglichen Leben an Friedrichsplatz auffällig wurden." (I8)

„..135 Spielplätze, die bevorzugt von Alkoholkranken, Obdachlosen und Jugendlichen aus den GUS Staaten angenommen werden." (I8)

„...Rechtsradikale haben Treffen im Wald abgehalten“ (I10)

„...dann war es also so schwierig, dass sich sowohl im Einkaufszentrum des Stadtteils als auch in Tiefgaragen Jugendcliquen getroffen haben, die also dort überhaupt nichts zu suchen hatten, sich selbst und andere gefährdet haben und natürlich auch massiv gestört haben." (I14)

„Dann sind die natürlich auch alle alkoholabhängig, ja oder drogenabhängig, Alkohol ist ja auch eine Droge, am helllichten Tage setzen die sich einen Schuss auf der Bank und dann kommen da Familien vorbei oder Schulklassen und das ist eine Sache, die wir eigentlich nicht mögen." (I26)

Diese Beispiele spiegeln die Varianten wider, dass es durch den Aufenthalt von Problemgruppen an bestimmten, meist exponierten, Orten in den Großstädten zu diversen Konflikten kommt. Neben diesem Konfliktgrund stellt die Lärmbelästigung einen weiteren Faktor zur Auslösung von Konflikten dar. So werden in den Interviews Jugendliche beschrieben, die sich auf Spielplätzen aufhalten, dort durch laute Musik auffallen und stören (I6). Konflikte bringen in unterschiedlichen Städten große Ansammlungen von Menschen auf Freiflächen oder Plätzen, wo es zu Ruhestörungen mit den umliegenden Anwohnern kommt $(I 1 ; 16 ; 115)$.

In den Interviews wird weiterhin deutlich, dass der Aufenthalt von Personengruppen im Freien häufig zu einem konflikthaften Nachbarschaftsverhältnis führt:

\begin{abstract}
„Aber für die ist es einfach so, natürlich dadurch dass sie draußen leben im Sommer, wenn es warm ist, bis spät in die Nacht, die Kinder dabei sind, ist das natürlich potentiell für alle deutschen Nachbarn eine Provokation und eine Belästigung." (I1)

„....weil einfach diese Cliquen störten und die Anwohner haben an den Bürgermeister geschrieben,..." (I14)
\end{abstract}

Diese o. g. Konflikte mit verschiedenen Personengruppen sind die Folge von der NichtEinhaltung von Regeln, die das Zusammenleben untereinander ordnen.

\begin{abstract}
„Aber nur wenn da solche Randgruppen oder natürlich Jugendliche, die nach einem gewissen Grad von Alkoholkonsum auch nicht mehr sich an die Regeln, die sie eigentlich kennen, aber die Hemmschwelle einfach sinkt und dann kriegen sie das nicht mehr in den Griff." (I15)
\end{abstract}

Vor allem wird an diesem Punkt das Dealen mit illegalen Drogen angesprochen (I12; I16), denn hierbei wird durch illegales Verhalten gegen das Gesetz verstoßen.

Ein weiterer, häufig genannter, Konfliktgrund ist das Müllproblem. Ca. 16\% der gesamten 62 Nennungen bezüglich Konflikten mit Problemgruppen gaben als Grund die Müllproblematik an. 
„...Spuren, die auf den Grünflächen hinterlassen werden, wie Scherben, Spritzen, Hundekot, Dosen deuten auf o. g. RG hin." (19)

„Es gibt immer wieder Konflikte, wenn sich diese Menschen auffällig verhalten, d.h. wenn sie ihr Umfeld stören, indem sie Hinterlassenschaften unterschiedlichster Art, Verpackungsmüll und dergleichen, einfach sorglos damit umgehen und einfach liegenlassen..." (I15)

„Wir haben dann leider immer das Problem der Müllbeseitigung, weil also dort riesige Müllansammlungen sind, wir können da vorne gleich gerne mal an die Tonne gucken, wir haben da zwei Container mit zwei Kubikmeter Fassungsvermögen, die haben nicht gereicht den Müll aufzunehmen, den wir hier im Wald haben, die werden wöchentlich geleert und wir haben da teilweise riesige Mülllager." (123)

„...wir haben die Problematik mit Spritzen auf Kinderspielplätzen...“ (I24)

„....und das ist halt, na gut dass ist nicht gerade ein Ruhmesblatt, weil dann eben zunächst mal, später sind dann noch Spritzen dazu gekommen, aber als nur die Schwulen das genutzt haben, dann gebrauchte Präservative, Papiertaschentücher und so und da haben die Arbeiter natürlich keine große Lust...(dies zu entsorgen (Anmerk. d. Verf.)) (I17)

An diesen Interviewauszügen wird aufgezeigt, wie störend die einzelnen Institutionen und Gesprächspartner die Müllproblematik empfinden, und dass die Entsorgung zu Konflikten führt.

Hinzu kommt ein Konflikt, den Personengruppen mit Hunden hervorrufen. Es handelt sich dabei meist um Beschwerden über die Verdreckung durch Hunde oder die Angst der Bevölkerung vor freilaufenden Hunden auf öffentlichen Flächen.

„....so dass wir da ganz massive Schäden auch an der Anlage haben, auch mit den Hunden, Hundeverdreckung, Verkotung." (I3)

„...Problem mit freilaufenden Hunden..." (I12

„....oder wenn sie, die Leute haben ja meistens Hunde, fast alle, wenn sie aggressive Hunde haben oder zu viel, dann taucht das auf, dann tauchen Probleme auf." (I15)

„....Randgruppen, sagen wir mal, uneinsichtige Leute als Randgruppe, die Hunde frei laufen lassen oder solcher Art Dinge und da gibt es natürlich manchen Ärger. Ich habe jetzt vorgestern wieder einen aktuellen Fall gesehen, wo ein Rehbock durch Hundebisse so verunstaltet war, dass er im Wald verreckt ist." (I21)

Auf die Frage nach der Art des Konfliktes mit Problem- oder Randgruppen gaben die Interviewten noch weitere Kategorien an. Diese reichen von wilden Feuerstellen und großen „Zechgelangen“ (I23) bis hin zu Vandalismus von unterschiedlichen Personengruppen auf öffentlichen Plätzen und Einrichtungen.

„...Wilhelmsplatz haben wir eher das Problem der Trinker oben, so dass wir da ganz massive Schäden auch an der Anlage haben,..." (I3)

„....Jugendliche randalieren auf den Spielplätzen und Vandalismus spielt eine große Rolle, d.h. Zerstörung von Spielgeräten..." (19)

„Natürlich haben wir hier einen wahnsinnigen Vandalismus auch innerhalb der Stadt, so auch bei den Einrichtungen, Bänke, Schutzhütten, Papierkörbe oder sonstige Dinge, die 
andere normal nutzen, die treten dagegen und dann fällt der ganze Schamott raus und dann haben wir das wieder am Hals." (I21)

Vandalismus stellt in den unterschiedlichen Städten ein weitreichendes Problem dar, denn gerade die Zerstörung von Erholungseinrichtungen und Spielgeräten führt zwangsläufig zu Konflikten, da sie von verschiedenen Personengruppen, wie z.B. Kindern oder älteren Bürgerinnen und Bürgern, genutzt werden.

In den Interviews wurde die Frage an die Vertreter der einzelnen Institutionen gestellt, wie sie auf die Konflikte aufmerksam gemacht wurden. Die Antworten variieren hier sehr stark, denn z. T. nehmen eigene Mitarbeiter die Konflikte wahr, wie z. B. Müll oder Vandalismus $(I 3,112)$ oder Passanten machen auf Problemgruppen aufmerksam, wie Wohnungslose (I15), Rechtsradikale (I10) oder das Müllproblem (I23). Aber auch andere Institutionen tragen einen Teil zur Konfliktaufdeckung bei, wie z.B. das Ordnungsamt (I3) oder der Jugendsachbearbeiter der Polizei, der wahrnimmt, dass sich Jugendliche an ungeeigneten Orten im Stadtteil aufhalten und Kontakt mit den örtlichen Jugendzentrum aufnimmt (I14).

Den Ausführungen folgend, gibt es viele Varianten von Konflikten mit Personengruppen in den Großstädten, wobei als Hauptkonflikte im Forstsektor der Aufenthalt von Problemgruppen im Wald und damit einhergehend die Müllproblematik genannt werden können. Die Forstverwaltungen scheinen aber über die Konflikte wohl informiert zu sein, da betont wurde, dass meist die Revierleiter Bescheid wüssten (I15) oder aber Waldspaziergänger eine hilfreiche Informationsquelle für Probleme bzw. Konflikte seien (I15; 123). Die Information durch die Bürger lässt auf eine Identifikation derer mit „ihrem“ Wald schließen und zeigt, dass die Informationswege direkt verlaufen bzw. die Bürger direkt wissen, an wen sie sich im Problemfall wenden müssen.

\subsubsection{Charakterisierung der Randgruppen}

Nach der Analyse von Konflikten, die in den Großstädten bestehen, folgt die Charakterisierung von Problem- bzw. Randgruppen. Aus dieser Charakterisierung durch die Experten der einzelnen Institutionen ist es wichtig zu erkennen, um welche Personengruppen es sich handelt, mit denen die Institutionen fast täglich umgehen und welche Eigenschaften diesen Gruppen zugeschrieben werden.

„....also die Punker z.B., die ganz gerne in den Leineauen oder im Wäldchen wohnen oder zelten." (I1)

„Wir sind zu S., der ja eine Erdhöhle im Wald hat und da wirklich seit 10 Jahren in einer Erdhöhle wohnt..." (I1)

„Also die helfen sich ganz stark untereinander. Da ist auch egal wie alt, die Alten helfen den Jungen und die Jungen helfen den Alten, also das ist nicht so..." (I1) 
„Und die lebt ja auch nur im Grünen, die schläft da in den Büschen, da ist so ein alter Lüftungsschacht, wo warme Luft rauskommt und da schläft die im Winter. (I1)

„....es gibt Obdachlose und es gibt Obdachlose, es gibt also Obdachlose, die sind hier so, na wie sagt man, so Durchreisende oder die schlafen mal hier und schlafen mal da. Dann gibt es auch welche, die bauen sich richtig Zelte auf und machen sich es hier gemütlich..." (I26)

Bei diesen Textbeispielen handelt es sich um die Eigenschaften und Gewohnheiten von Obdachlosen. Nicht nur die hier aufgeführten Institutionen nannten den Kontakt mit Obdachlosen, sondern auch andere befragte Einrichtungen, die aber keine weiterführende Charakterisierung der Personengruppen im Verlauf des Gesprächs lieferten.

Eine weitere Personengruppe, mit der sich unterschiedliche Institutionen auseinandersetzen sind Straffällige oder von Straffälligkeit bedrohte Menschen. Ihre Lebenssituation und Umstände werden folgend beispielhaft von einer Institution beschrieben.

„Die Lage der Leute kann „idealtypisch“ folgend beschrieben werden: kein Konto mehr; wenn eine Wohnung da ist, dann weiß man nicht, wie man sie bargeldlos bezahlen soll; wer zahlt, das Sozialamt oder das Arbeitsamt; Schulden und überall warten Gläubiger; Zukunftsplanung wegen Gerichtsverhandlung schwierig." (I2)

An dem folgenden Beispiel werden die Zusammensetzung und die Struktur von Jugendcliquen wiedergegeben, die sich auch auf weitere Kontakte von Institutionen mit Jugendlichen übertragen lässt.

„Die Stabilität der Cliquen war wesentlich geringer als wir gedacht haben und entsprechend, das fluktuierte, das waren zwar alles junge Leute, die sich kannten zwischen ca. 14-15 und 18-19, weil die alle im Stadtteil aufgewachsen sind, aber es war durchaus nicht so, das diese Clique ganz fest gefügt war mit Meinungsführern, mit einer Struktur, die sie über einen längeren Zeitraum auch so in dieser Form gehalten hätte." (I14)

„Aber auch da hatten wir auch immer das Problem, dass rivalisierende Gruppen aus dem Stadtteil oder auch nur mal Konflikte, die sich zwischen zwei drei oder vieren ergeben hatten, dazu führten, dass man dann den Anderen, die gerade auf dem Platz waren, dann Einrichtungen zerstörte." (I14)

Eine weitere Personengruppe, mit der sich einige Institutionen beschäftigen, sind Langzeitarbeitslose.

„...entweder sind es ganz alte oder ganz junge Leute, die praktisch nie eine Ausbildung gemacht haben aus welchen Günden auch immer, von der Schule gleich in die Arbeitslosigkeit oder die Sozialhilfe gegangen oder eben ältere Leute, die auch wieder schlecht vermittelbar sind, weil eben alt und gebrechlich." (I26)

Dieses Beispiel fasst grob zusammen, warum sich eine große Zahl Menschen in der Situation Arbeitslosigkeit befinden. Diese Charakterisierung trifft auch auf die übrigen Institutionen mit dieser Klientel zu. 
Nach diesen oben aufgeführten deskriptiven Faktoren über die Konflikte und Kontakte einzelner Institutionen mit Problem- oder Randgruppen wird deutlich, dass Handlungsbedarf besteht. Diesen Handlungsbedarf haben soziale und karitative Einrichtungen längst erkannt und Projekte und Hilfsmaßnahmen eingeleitet. Ziel dieser vorliegenden Arbeit ist zu zeigen, ob der Forstsektor auch über ein Potential an Hilfsmaßnahmen verfügt und einen Beitrag zur integrativen Arbeit leisten kann. Hierzu gilt es die aufgestellten Hypothesen auf Basis der Experteninterviews zu überprüfen.

\section{2 Überprüfung der Hypothese I}

Hypothese I: a) Der Wald in urbanen Räumen besitzt ein großes Potential für soziale Nachhaltigkeit und soziale Integration.

b) Aufgrund ihres eingeschränkten Verständnisses der sozialen Nachhaltigkeit erschließt die Forstverwaltung dieses Potential nur in geringem Umfang.

\subsubsection{Lösungsansätze allgemein}

Einleitend wurde beschrieben, dass Konfliktpotential mit Problem- und Randgruppen vorhanden ist. Aus den Interviews kann eine Reihe von Lösungsansätzen dieser Konflikte ausgemacht werden. Hierbei handelt es sich zunächst um generelle Aussagen, die von den Institutionen im Bezug auf die Konfliktfelder benannt haben. Zum einem wurde hier schon die Kooperation als Lösungsansatz hervorgehoben.

„Ohne die Verknüpfung, möglicherweise durchaus ohne diese Persönlichkeiten, die daran mitgedacht haben, wäre es nicht gegangen." (I14)

Ein weiteres Instrument zur Konfliktlösung unterschiedlichster Probleme stellen die „Runden Tische“ dar. In vielen der untersuchten Großstädte wurde dieser Regelungsmechanismus angewandt.

„...AG Drogen: Dienstagsrunden alle 2 Monate (...) Teilnehmer: Polizeipräsident, Jugenddezernent, Sozialdezernent und gesamte Ämterbereich mit der Drogenhilfe Nordhessen e.V., Uni, staatliches Schulamt, Schulverwaltungsamt, Wohnungsamt, Sozialamt, Jugendamt, Gesundheitsamt (...) wo tauchen Probleme auf und wie kann dies gemeinsam präventiv gelöst werden." (I8)

„Und dann ist also zusammen mit einem runden Tisch in dem Stadtteil die Fragestellung aufgekommen, was können wir tun, wenn hier nicht die Möglichkeit besteht durch zusätzliche Sozialarbeiter diese Jugendlichen zu betreuen." (I14)

„Ja, das entwickelt sich dann so, dass man dann solche Arbeitsgruppen oder runde Tische hat, das entwickelt sich weiter, vielleicht eine Spezialität aus Freiburg." (I15) 
Aus den Interviews wird deutlich, dass die Institutionen das Instrument als adäquat ansehen, um die bestehenden Probleme zu lösen. In einem weiteren Gespräch wurde auch festgestellt, dass die Einrichtung von „Runden Tischen“ nicht zur Konfliktlösung beiträgt und kein Ergebnis hervorbringt. Es handle sich hierbei nur um einen inszenierten Prozess für die Presse (110). Dies Beispiel zeigt, dass es sich bei den „Runden Tischen“ nicht um ein „Allheilmittel“ handelt, mit dem jegliche Art von Problem zu lösen ist. Die weitere Nennung von Ansätzen zur Konfliktlösung, z.B. durch Gespräche bzw. Kommunikation zeigen folgende Textauszüge:

„So, dann hat es eben einen Ortstermin gegeben mit dem zuständigen Revierleiter und also mit einem Vertreter aus der Szene und mir und dann haben wir uns darauf geeinigt, dass da eben zwei Zufahrten offen bleiben und dass also auch nach wie vor das als Treffpunkt möglich ist". (I17)

Wir rechnen ja damit, dass Orte, die überwacht sind, wie die Grilleinrichtungen, die wir haben, zumindest ein Regulativ haben, dass mal einer guckt und sagt, benehmt euch oder so nicht, dass das auch mal gesagt wird. (I21)

„....ich sage mal, irgendwo hat man auch Mitleid mit diesen Menschen, das sind ja wirklich die Ärmsten der Armen dann auch, ich rede mit innen und sage, dass sie dann bitte am nächsten Tag ihre Sachen packen und weiterziehen und sich einen anderen Platz suchen, das machen sie dann in der Regel auch." (I23)

Diese Form der Konfliktlösung stellt, nach Meinung der Verfasserin, die Basis für eine Problemlösung dar. In einigen Fällen kann es durch ein Gespräch mit den Konfliktpartnern schon zu einer Lösung kommen bzw. das Problem direkt behoben werden. Geschieht dies in Konfliktsituationen nicht, ist die Kommunikation der Grundstein für weitere Maßnahmen der Konfliktregelung. Weiterhin decken die Interviews Lösungsansätze zur Konfliktregelung auf, die unter dem Stichwort Vertreibung zusammengefasst werden können. Die Personengruppen, die das Stadtbild auf verschiedenen öffentlichen Plätzen stören, werden vertrieben. Simon (2001, S. 14) beschreibt dieses Phänomen der Vertreibung mit dem Beispiel des "Junkie-Joggings", in dem versucht wird, Drogenabhängige aus dem öffentlichen Raum zu vertreiben, was zur Verlagerung der Szene führte, nicht aber zu deren Auflösung. Es werden so keine Alternativen zum Aufenthalt geboten, sondern eine zeitliche und räumliche Verlagerung der Konflikte erreicht. Da durch die Vertreibungstaktik das Problem der Öffentlichkeit mit den Problem- bzw. Randgruppen nicht gelöst wird, kann kaum von einem Problemlösungsmechanismus gesprochen werden. Auffällig ist die häufige Nennung dieses Phänomens in den Interviews, was darauf schließen lässt, dass einige der befragten Institutionen diesen Mechanismus als lösungsorientiert ansehen. Die Institutionen, die diesen Ansatz verfolgen gehören allerdings nicht zu dem Sozialsektor, sondern zählen eher zu den öffentlichen „Grünverwaltungen“ wie z.B. Grünflächenamt, deren Aufgabe in der Erhaltung der Flächen gesehen wird und die sich nicht in der Lage sehen, geeignete Alternativen zum Aufenthalt der Personengruppen zu bieten. Mit der Vermeidung von Aufenthaltskonflikten geht auch der Rückbau von Erholungseinrichtungen einher, z.B. 
werden Bänke von öffentlichen Plätzen entfernt und durch weniger „bequeme“ Drahtsessel ersetzt oder Einrichtungen direkt im Wald werden modifiziert, um diese als Treffpunkt bewusst unattraktiver zu machen.

„....Fußgängerzone, da hatten wir auch mal eine Zeit lang das Problem, dass welche betrunken auf den Bänken lagen, dann haben wir dann die Bänke umgewandelt in solche Stühle, wo man nicht mehr so bequem drauf liegen kann, solche Drahtgeflechte." (I3)

„....seitdem wir diese Modifikation Bank, also im 90Grad Winkel Bänke zu stellen, wohlmöglich noch ein Tisch dazwischen, lösen wir auf, sukzessive, machen wir nicht mehr und haben dadurch auch mehr Ruhe." (I21)

„Bloß das wird eben alles geduldet vom Staat, ja, die sagen am Bahnhof Zoo, da muss es sauber sein, da treiben sie sie weg, bloß sie sind ja nicht weg, die sind bloß woanders, das ist eine Verlagerung des Problems." (I26)

In einer der untersuchten Städte werden auch Grillplätze zurückgebaut, weil dort ein Sicherheitsrisiko in der Brandgefahr für die umliegenden Anwohner besteht. Die Situation hat sich an Wochenenden so zugespitzt, dass bestimmte ausländische Personengruppen diese Plätze okkupieren, zudem Müll und Lärm so zugenommen haben, dass sich die Ortspolitik entschieden hat, diese Grillplätze drastisch zu reduzieren (I23). In den meisten Großstädten wird der Konflikt Aufenthalt von Personengruppen im öffentlichen Raum versucht mit sozialer Kontrolle zu lösen. Dadurch dass Mitarbeiter einzelner Ämter oder die Polizei Präsenz an markanten Orten zeigen, sollen die Problemgruppen auf andere Aufenthaltsorte ausweichen (19; 112; 123). In einigen Fällen konnte ein Nutzungskonflikt durch eine Abstimmung der Interessen erreicht werden, d.h. eine Nutzergruppe verlagerte ihren Aufenthaltsort zugunsten einer anderen Gruppe (I15, I17).

Aus den oben genannten Konflikten, deren Schwerpunkt in der Nutzung von und dem Aufenthalt auf öffentlichen Plätzen bzw. Grünräumen liegt, kann auf die Ansprüche von sozialen Rand- bzw. Problemgruppen überleitet werden. In erster Linie handelt es sich um den Anspruch nach Treffpunkten im Freien. In den Interviews werden unterschiedliche Arten von Treffpunkten beschrieben. Beispielsweise sind Grünräume Treffpunkte für Ausländer, die es gewohnt sind, einen Teil ihres Lebens ins Freie zu verlagern (19) oder diese Freiräume können als informelle Treffpunkte genutzt werden, um sich der sozialen Kontrolle zu entziehen (I11). Weiterhin treffen sich auch Jugendliche im Wald, die sonst im Stadtteil negativ auffallen (I14). Ein weiteres Beispiel für den Anspruch an Treffpunkte im Freien sind Homosexuellentreffpunkte, d.h. Grünflächen auf denen sich abends in der Dunkelheit zur Kontaktaufnahme getroffen wird (I17). Als wichtiger Anspruch von Problemgruppen an den Freiraum kann in der Wohnraumerweiterung gesehen werden. Hierbei handelt es sich in den meisten Fälle um ausländische Bevölkerung, vornehmlich aus den südlichen Ländern, die einen Ersatz für die kleinen Wohnungen suchen oder sich dadurch ein Stück Freiheit schaffen wollen.

„Aber das ist ein Ersatz für eine zu kleine Wohnung und das ist auch eine Lebenserfahrung, die man aus dem Süden mitbringt. Man verlegt das Leben nach 
draußen, wenn es halt in der Wohnung stickig und eng ist, dann macht man das ganze draußen." (I1)

„Das ist ein Freiraum, den man nutzen kann, der einfach da ist, wo man genau weiß, dass die Polizei nicht viel Theater macht." (I1)

Weiterhin zeigen die Interviews die Nutzung von Holz zum Heizen als Anspruch von Randgruppen (I1), d.h. sie halten sich im Grünraum nur auf, um mit einem Einkaufswagen in den Wald zu fahren und dort Brennholz zu sammeln. Außerdem besteht der Anspruch von Wohnungslosen, z.B. den Wald zu ihrer Wohnstätte zu machen (I12).

Um zu erkennen, ob die Konflikte durch geeignete Maßnahmen gelöst und Ansprüche von Personengruppen durch diese erfüllt werden können, werden die Maßnahmen zur Integration von sozialen Rand- bzw. Problemgruppen analysiert.

\subsection{Integrationsmaßnahmen}

Bei der Frage nach integrativen Maßnahmen der einzelnen Institutionen geht es um Projekte, die im Wald bzw. mit Bezug zum Wald stattfinden. Eine erste Übersicht der verschiedenen Maßnahmen in den untersuchten Großstädten bieten Tabellen. Aufgeführt sind hier zunächst alle Aktivitäten der befragten Institutionen, die zur Integration beitragen und auf Grünflächen oder im Wald ablaufen bzw. stattfinden. Die Beschreibungen der Maßnahmen finden nach den Fallstudien getrennt statt. 


\subsubsection{Fallstudie Göttingen}

Tabelle 13: Institutionen in Göttingen, deren Maßnahmen und Partner

\begin{tabular}{|c|c|c|}
\hline \multicolumn{3}{|c|}{ Göttingen } \\
\hline Institution & Maßnahme / Projekt & Partner \\
\hline Fachbereich Stadtgrün & Platz für Wagenburg & k.A. \\
\hline Straßensozialarbeit & $\begin{array}{l}\text { Arbeitserprobungen für } \\
\text { Sozialhilfeempfänger z.B. } \\
\text { Müllsammeln }\end{array}$ & - Sozialamt, Jugendämter \\
\hline KiK e.V. (Kontakt in Krisen) & $\begin{array}{l}\text { Agrar- und Naturschutzprojekt für } \\
\text { Straffällige und von Straffälligkeit } \\
\text { Bedrohter, Drogenabhängige, } \\
\text { Langzeitarbeitslose, z.B. } \\
\text { Holzeinschlag (Brennholz), } \\
\text { Freischneidearbeiten, (zusätzliche } \\
\text { und niederschwellige Arbeit) }\end{array}$ & $\begin{array}{l}\text { - Landkreis Göttingen } \\
\text { (Naturschutzarbeiten) } \\
\text { - Stadtforstamt Göttingen } \\
\text { - Privatforst }\end{array}$ \\
\hline Fachbereich Sozialplanung & $\begin{array}{l}\text { Projekt „Soziale Stadt“: } \\
\text { Stadtteilsanierung in Grone, } \\
\text { Grünflächengestaltung als } \\
\text { Begegnungsstätte }\end{array}$ & $\begin{array}{l}\text { - Quartiersmanagement } \\
\text { - Stadt Göttingen (Bauamt, } \\
\text { Stadtplanung etc.) }\end{array}$ \\
\hline Stadtforstamt & $\begin{array}{l}\text { - Arbeiten für Langzeitarbeitslose } \\
\text { - Arbeiten für Suchtkranke } \\
\text { - Waldpädagogik für Behinderte } \\
\text { - Brennholzverarbeitung }\end{array}$ & $\begin{array}{l}\text { - Sozialamt } \\
\text { - Arillus GmbH, } \\
\text { Therapieeinrichtung Söddereich } \\
\text { - KiK e.V. }\end{array}$ \\
\hline Jugendamt & $\begin{array}{l}\text { - Spielplatzgestaltung } \\
\text { - Ferienprogramm }\end{array}$ & k.A. \\
\hline Arillus $\mathrm{GmbH}$ & $\begin{array}{l}\text { - Outdoor Programm für } \\
\text { Suchtkranke } \\
\text { - Waldpädagogik für Behinderte }\end{array}$ & $\begin{array}{l}\text { - Stadtforstamt Göttingen, } \\
\text { Forstamt Braunlage, } \\
\text { Therapieeinrichtung Södderich }\end{array}$ \\
\hline
\end{tabular}

Die einzelnen Maßnahmen zeigen ein breit gefächertes Angebot für unterschiedliche Personengruppen zur Integration. Da zur Beantwortung der Hypothese vor allem die Maßnahmen des Stadtforstamtes von Bedeutung sind, sollen diese näher beschrieben werden. In Göttingen bietet das Stadtforstamt in Ausnahmefällen Arbeitsgelegenheiten für Langzeitarbeitslose. Dieses Programm sieht vor, die Menschen, die lange nicht in Arbeit waren, durch Arbeitseinsätze wieder an regelmäßige Zeitabläufe zu gewöhnen (I5). Im Vordergrund steht hier Wiedereingliederung in den Arbeitsmarkt durch Qualifizierung der Maßnahmenteilnehmer (z.B. Umgang mit Motorsägen).

Weiterhin arbeitet das Stadtforstamt mit der Arillus $\mathrm{GmbH}$ in einem Beschäftigungsprojekt für Suchtkranke zusammen. Hierbei sollen die Teilnehmer, die aus der Therapieeinrichtung Södderich kommen, durch harte Arbeit (I5) und Outdoor-Aktivitäten, wie das Übernachten im Wald, eine Schlafstätte selbst herrichten, eigenverantwortlich für die Mahlzeiten sorgen, an ihre Grenzen geraten und körperliche Arbeit, Teamfähigkeit und Verantwortung wieder erlernen. Dieses Projekt basiert auf klaren therapeutischen Ansätzen für die meist Drogenabhängigen aus einer Suchtklinik nahe Göttingen. Die Besonderheit dieser Maßnahme besteht darin, dass die Teilnehmer weit ab von der Zivilisation diese Naturerfahrungen machen können. Da dies meist im Winter stattfindet und der Göttinger 
Wald nicht schneesicher ist (17), wird seit einiger Zeit auf das Forstamt Braunlage ausgewichen, wo die Kriterien der Schneesicherheit und Ferne von Zivilisation gegeben sind. Die Anfänge dieses Projektes liegen allerdings in der Zusammenarbeit mit dem Forstamt Göttingen, denn im Göttinger Stadtwald hat zum ersten Mal eine solche Maßnahme stattgefunden.

Zudem arbeiten Arillus GmbH und das Stadtforstamt in der Waldpädagogik zusammen (I5, 18). Es handelt sich hierbei um die Arbeit mit Behinderten, die für eine Bildungsintervention in den Wald kommen und dort Aktionen erleben, die z.B. die Motorik stärken und Sinne wie Tasten oder Hören schärfen. Die Veranstaltungen mit diesen Personengruppen sind von einer hohen Flexibilität der Betreuer, einer hohen Betreuerzahl und dem Einsatz der Forstamtsmitarbeiter gekennzeichnet. Dieser Einsatz äußert sich z.B. im Pflanzen oder Fällen von Bäumen, um einen ganzheitlichen Eindruck der Forstarbeit zu vermitteln (I5, II7). Die Dauer der einzelnen Projekte zur Integration spielt eine Rolle für deren Erfolg. Bei den o.g. Maßnahmen handelt es sich größtenteils um kurzfristige Interventionen, wie die Waldpädagogikangebote, die nur einen Tag bis hin zu einer Woche andauern. Eine Studie belegt, dass positive Effekte von kurzzeitpädagogischen Bildungsinterventionen im Naturund Umweltschutzinteresse von Kindern und Jugendlichen ohne Behinderung festgestellt werden können (Bittner, 2003). Da die Wahrnehmung von Personen mit Behinderungen in der Natur noch verstärkt ist, kann auch hier von einem nachhaltigen Effekt ausgegangen werden. Galuske (2005, S. 252) beschreibt hierzu, dass durch die Erlebnispädagogik Ziele wie z.B. die Selbständigkeit, eigene Grenzen und Ressourcen zu entdecken, Gefühle wahrzunehmen und auszudrücken, erreicht werden können. Die Zielgruppen können alle gesellschaftlichen Gruppen umfassen, von Schülern, alten Menschen, Drogenabhängigen bis hin zu chronisch bzw. unheilbar Kranken. Die Beschäftigungsprojekte, die in Zusammenarbeit mit dem Stadtforstamt ablaufen, sind ebenfalls von kurzfristiger Dauer, d.h. bis zu einem halben Jahr. Die Interviewten der Institution KiK e.V. äußern sich zu der Dauer dieser Maßnahmen ambivalent:

„Seit diesem Jahr enden die Projekte nach einem $1 / 2$ Jahr (...) reicht manchmal für dieses Klientel aus." (I2)

„1-jahres Projekt beinhaltet ein besseres Betreuungsangebot (...) bei kürzerer Dauer kann man kaum Personen aufnehmen, die einen hohen Betreuungsbedarf haben..." (I2)

Hieran wird deutlich, dass die Betrachtungsweise für die Beschäftigungsprojekte von Einzelfällen abhängig ist. Für einige Menschen, die lange nicht in Arbeit waren, reicht ein kurzer Zeitraum aus, um den Anschluss an das Arbeitsleben zu erhalten. Bei Personen, deren Problem nicht nur die Arbeitslosigkeit ist, bedarf es einer längerfristigen Maßnahme, um eine ganzheitliche Änderung der Lebensbedingungen zu schaffen.

Werden Maßnahmen betrachtet, die zur sozialen Integration beitragen, stellt sich die Frage nach dem Erfolg solcher Maßnahmen. Der Erfolg der Beschäftigungsmaßnahme in 
Zusammenarbeit mit dem Stadtforstamt und KiK e.V. wird von den Vertretern der sozialen Einrichtung in der Qualifikation der Teilnehmer gesehen, was sich in der Ausstellung von Zertifikaten und Zeugnissen über die Lehrgänge niederschlägt (I2). Weiterhin sollten die Folgeerscheinungen von Straffälligkeit und Langzeitarbeitslosigkeit, wie z.B. Schulden, planbar sein bzw. es sollte ein Konzept bestehen, diese abzubauen (12). Außerdem sollten Bewerbungsunterlagen vorhanden sein. Im Bestfall sollten sich die Teilnehmer an einer Beschäftigungsmaßnahme, nach Beendigung dieser, in Arbeit befinden. Diese Vermittlungszahlen sind sehr gering, da die allgemeine Situation auf dem Arbeitsmarkt äußerst angespannt ist und Personen, deren Lebensläufe Haftstrafen und Drogenkonsum beinhalten, häufig nicht vermittelt werden können (I2). Der Erfolg der Outdoor-Erfahrung mit Drogenabhängigen im Wald wird als nachhaltig beschrieben (17). Hier handelt es sich um Grenzerfahrungen, die von den Teilnehmern gemacht werden, wie die körperliche Arbeit, die für einen Suchtkranken schon eine Grenze darstellen kann. Es wird ihre Teamfähigkeit und das Verantwortungsbewusstsein geschult und der entspannte Umgang in der Therapieeinrichtung untereinander als Erfolg gewertet. In der Studie "Ich - Draußen“ beschreiben Gouder et al. (2003, S.93) den Erfolg von Outdoor-Aktionen zudem in der Reflektion der Naturerfahrungen in der Therapieeinrichtung. Diese Erfahrungen lassen sich auf individuelle Lebenssituationen übertragen und decken gesellschaftliche Potentiale Einzelner auf (ebd., S. 94).

Alle befragten Institutionen zeigen durch die Durchführung der einzelnen Projekte einen Einsatz für Problemgruppen bzw. ein Interesse für diese Personengruppen und die Verbesserung der Situationen derer. Das Göttinger Stadtforstamt macht die eigene Position deutlich, indem es sich als Dienstleister versteht und solche Projekte außenwirksam darstellt (I5) und den sozialen Auftrag der Institution erkennt.

„Die einzige Chance ist, dass man sich als Dienstleister darstellt und rechtfertigt und das natürlich auch zeigt. Also sie brauchen solche Gruppen und solche Arbeit, um nach außen zu zeigen, wir haben einen sozialen Auftrag unter vielen anderen Aufträgen auch." (I5)

Die Verbindung von sozialen Problemgruppen und den Vertretern des Forstsektors wird von Arillus $\mathrm{GmbH}$ als schwierig beschrieben, da häufig Berührungsängste von Seiten der Forstleute bestehen (17).

„Die Förster trauen sich nicht an Problemgruppen heran.“ (I5)

Zusammenfassend kann für die Projekte und Maßnahmen zur Integration von Problem- bzw. Randgruppen in Göttingen festgestellt werden, dass die kommunale Forstverwaltung einen Beitrag zur sozialen Integration leisten kann, indem sie den verschiedenen Problemgruppen Akzeptanz gegenüberbringt und diese toleriert. Es fällt bei der Analyse der Ergebnisse aber auf, dass die Bereitschaft zur sozialen Integration beizutragen fast ausschließlich in der Bereitstellung von Flächen gesehen wird. Die Forstseite ergreift z. Zt. keine dauerhaften 
Maßnahmen zur sozialen Integration, die z.B. die gesellschaftliche Stellung durch das Angebot eines Arbeitsplatzes im Forstbetrieb der Personen verbessern würde. Weiterhin ist die Forstsektor nicht in der Lage, die weitgreifenden Mechanismen der Sozialintegration zu überblicken, wie z.B. eine dauerhaft angelegte Hilfe (Beschäftigung) für Suchtkranke, die einen Rückfall verhindern könnte. Für alle aufgeführten Projekte muss sich die Forstverwaltung der Hilfe von anderen Institutionen bedienen, die in den einzelnen Projekten vornehmlich die Durchführung der Angebote übernehmen. Die Hypothese I kann nach der Analyse der Projekte für die Fallstudie Göttingen bestätigt werden, denn das Potential des Waldes im Hinblick auf soziale Integration kann nachgewiesen werden. Es herrschen auf der Seite der Forstverwaltung dennoch ein eingeschränktes Verständnis von sozialer Integration und damit auch ein eingeschränkter Beitrag zur sozialen Nachhaltigkeit. Das Verständnis der Forstverwaltung geht nicht in die Richtung einen entscheidenden Beitrag zur sozialen Nachhaltigkeit leisten zu wollen oder zu können, denn die aktive Einbringung in die Projekte kann nicht nachgewiesen werden. Ebenso wird das Potential nicht ausreichend genutzt bzw. kann nicht ausreichend genutzt werden, denn es handelt sich bei den Arbeitsprojekten für Langzeitarbeitslose oder Straffällige nicht um feste Bestandteile der Aufgabenwahrnehmung der Forstverwaltung, d.h. kein dauerhaftes Angebot. Anders wird die Umweltbildung für Kinder und Jugendliche gesehen. Diese ist gesetzlich verankerte Aufgabe der Forstverwaltung (NWaldLG, §15(4)), wobei hier erwähnt werden muss, dass dieser Aufgabenbereich ausgelagert und von anderen Institutionen übernommen wird. 


\subsubsection{Fallstudie Kassel}

Tabelle 14: Institutionen in Kassel, deren Maßnahmen und Partner

\begin{tabular}{|c|c|c|}
\hline \multicolumn{3}{|c|}{ Kassel } \\
\hline Institution & Maßnahme / Projekt & Partner \\
\hline Umwelt- und Gartenamt & - Ableistung von Sozialstunden & $\begin{array}{l}\text { - Sozialamt, Gerichtshilfe, } \\
\text { Jugendamt }\end{array}$ \\
\hline Sozialamt & $\begin{array}{l}\text { - Akquirierung von Arbeit für Nutzer } \\
\text { illegaler Drogen, z.B. auf } \\
\text { Grünflächen }\end{array}$ & $\begin{array}{l}\text { - Naujob (gemeinnützige } \\
\text { Einrichtung) }\end{array}$ \\
\hline Forstamt & $\begin{array}{l}\text { - Grillplätze } \\
\text { - Zeltplatz für Jugendliche } \\
\text { - Asphaltwanderweg am Herkules } \\
\text { - Umweltbildung } \\
\text { - Waldlehrpfad für Behinderte } \\
\text { - Beschäftigungsprojekt für } \\
\text { Sozialhilfeempfänger } \\
\text { - Arbeitseinsatz von Jugendlichen }\end{array}$ & $\begin{array}{l}\text { - Arillus GmbH } \\
\text { - Marburger Blindensportbund } \\
\text { - AGIL mbH } \\
\text { - Schule für Schwererziehbare } \\
\text { Jugendliche }\end{array}$ \\
\hline Jugendamt & $\begin{array}{l}\text { - Beteiligung von Kindern und } \\
\text { Jugendlichen bei der Planung von } \\
\text { z.B. Gestaltung einer Grünfläche in } \\
\text { der Nähe einer Schule }\end{array}$ & - Ämter der Stadt (Bauamt) \\
\hline Staatl. Schlösser + Gärten & $\begin{array}{l}\text { - Arbeitseinsatz von Jugendlichen } \\
\text { zur Bekämpfung der } \\
\text { Jugendarbeitslosigkeit } \\
\text { - „Arbeit statt Strafe“ } \\
\text { - „Hessenpraktikum“, } \\
\text { Jahrespraktikum für Jugendliche }\end{array}$ & $\begin{array}{l}\text { - freie Einrichtungen u. Stadt } \\
\text { Kassel } \\
\text { - Gerichtshilfe } \\
\text { - Arbeitsamt (Agentur für Arbeit) }\end{array}$ \\
\hline $\begin{array}{l}\text { AGIL mbH (Gemeinnützige } \\
\text { Arbeitsförderungsgesellschaft im } \\
\text { Landkreis Kassel mbH) }\end{array}$ & $\begin{array}{l}\text { - Beschäftigungsprojekte für } \\
\text { Sozialhilfeempfänger } \\
\text { (Obstbaumpflege, } \\
\text { Freischneidearbeiten) } \\
\text { - Ausbildung statt Sozialhilfe für } \\
\text { Jugendliche }\end{array}$ & $\begin{array}{l}\text { - Landkreis Kassel } \\
\text { - Forstamt Kassel, Forstamt } \\
\text { Wolfhagen } \\
\text { - Sozialamt } \\
\text { - Agentur für Arbeit }\end{array}$ \\
\hline
\end{tabular}

Die Maßnahmen und Projekte in Kassel zeigen eine Bandbreite von integrativen Aktionen für problematische Personengruppen. Auch hier soll zur Hypothesenüberprüfung der Fokus auf den Projekten liegen, die in Verbindung mit dem Forstamt bzw. der Forstverwaltung stattfinden.

Neben der Bereitstellung von Grillplätzen hat das Stadtforstamt Kassel einen Zeltplatz für Jugendliche eingerichtet. Die Einrichtung eines solchen Platzes zeigt eine Reaktion des Forstamtes auf die Nachfrage (110). Die Asphaltierung eines Wanderweges zum HerkulesDenkmal zeigt ebenfalls eine Reaktion auf die Bedürfnisse von Menschen, die nicht in der Lage sind auf den unausgebauten Wegen durch den Wald zu wandern (z. B. Menschen, die an den Rollstuhl gebunden sind).

Das Stadtforstamt Kassel arbeitet in der Umweltbildung ebenfalls (s. Göttingen) mit der Arillus $\mathrm{GmbH}$ zusammen. Hier wird von Seiten des Forstamtes deutlich, dass ein Teil des Bildungsbereiches vergeben wird, da es zeitlich und personell nicht zu leisten ist („outsourcing“). Bei der Gestaltung z.B. einer ganzen Bildungswoche im Wald ist dem Forstamt wichtig, dass die eigenen Interessen, nämlich eine „moderne - multiple use Forstwirtschaft" (I10) vertreten wird. 
Als reines Integrationsprojekt kann die Anlage eines Waldlehrpfades für Menschen mit Behinderungen angesehen werden. Dieser wurde in der Nähe eines Altenheimes und in Zusammenarbeit mit dem ,Marburger Blindensportbund' realisiert. Die Nachfrage nach diesem Waldlehrpfad bestand, da gerade Menschen mit Behinderungen, z.B. Blinde oder Menschen in Rollstühlen, einen speziellen Zugang zum Wald benötigen. Beispiele für einen solchen Zugang sind befestigte Wege und Stationen, an denen die Menschen Information des Waldes über die Sinne Hören und Tasten erfahren (I10).

Einen weiteren Schwerpunkt der Projekte mit der Beteiligung des Forstamtes machen die Beschäftigungsprojekte aus. In Zusammenarbeit mit einer Schule für schwererziehbare Jugendliche fanden Arbeiten im Wald, wie Astungen und Pflegearbeiten statt (I10).

Die Gesellschaft AGIL mbH hat die Beschäftigungsförderung im Landkreis Kassel zur Hauptaufgabe. Zu diesem Zweck bestand eine Zusammenarbeit mit dem Stadtforstamt in Kassel. Hierbei wurden vor allem Sozialhilfeempfänger, mit unterschiedlichen persönlichen Hintergründen (z.B. Migration), in Arbeitsprojekten, die Freischneidearbeiten und Pflegemaßnahmen beinhalteten, beschäftigt. Ziele dieser Maßnahmen sind die Qualifizierung und Wiedereingliederung der Sozialhilfeempfänger in den Arbeitsmarkt (I13). In den Interviews wurde betont, dass es sich bei den Arbeiten immer um zusätzliche Arbeiten handelt und somit das Arbeitsfeld der Waldarbeiter nicht eingeschränkt wurde (I13). Ca. 18 Monate arbeiten die Personen in diesen Maßnahmen auf den Flächen des Landkreises und des Forstamtes, so dass von einem langfristig (d.h. über ein Jahr Laufzeit) angelegten

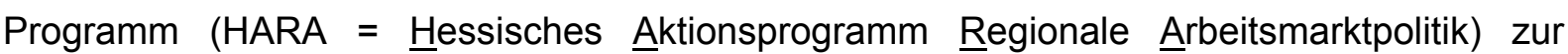
Beschäftigungsförderung gesprochen werden kann, bei dem die integrativen Erfolge in der Qualifizierung gesehen werden (113). Zudem ermöglicht das Zeugnis, das AGIL mbH am Ende der Maßnahme ausstellt, einen besseren Einstieg in das Berufsleben. Als integrativer Erfolg wird die Vermittlung der Maßnahmenteilnehmer in Praktika gesehen, aus denen sich schon Arbeitsverhältnisse entwickelt haben (113). Die Vermittlung oder Übernahme in Arbeitsverhältnisse ist das Ziel fast aller Beschäftigungsmaßnahmen. So beschreibt auch die Institution Staatliche Schlösser und Gärten Kassel als integrativen Erfolg die Vermittlung eines Straffälligen, der eine 25 jährige Haftstrafe abgeleistet und eine Arbeitsbeschaffungsmaßnahme in der Institution geleistet hat, in ein Arbeitsverhältnis bei einer Spedition (112). Auch die Übernahme von Praktikanten in ein Ausbildungsverhältnis wird als erfolgreiche Integration gewertet (112). Den Aspekt des Respekts und der Tolerierung gegenüber Problemgruppen, speziell Arbeitslosen jeglicher Altersstufe, wird vor allem in der Äußerung des Interviewpartners der Staatlichen Schlösser und Gärten deutlich.

„....solchen Leuten sollte die Chance geboten werden (...) es ist immer ein Risiko und eine Gratwanderung (...) aber da sehe ich Möglichkeiten." (I12) 
Es gibt demnach die Möglichkeiten, Langzeitarbeitslose, Sozialhilfeempfänger oder Jugendliche auf den Grün- und Freiflächen zu beschäftigen, sie in die Mitarbeitergruppen einzubinden und innen einen besseren Start in das Arbeitsleben zu ermöglichen. Die interviewte Person gibt aber auch zu bedenken, dass diese Problemgruppen eine Belastung für den Arbeitsablauf darstellen können und dass die Aktionen bei betrieblicher Überlastung nicht mehr durchführbar sind (112). Dass eine Nutzung und Erholung aber gerade für Problemgruppen auf Wald- und Grünflächen wichtig ist, wird durch folgendes Zitat ausgedrückt:

„Randgruppen können sich ja auch im Wald erholen, z.B. die Arbeitslosen, die gefrustet sind, die gehen in das „Psychotop" Wald ..." (I10)

Auch die Öffnung von Parkanlagen zur Nutzung und Erholung zieht das Auftreten von Problemgruppen mit sich. Dies nicht zu unterbinden, sondern eine Nutzung im angemessenen Rahmen zu tolerieren, zu akzeptieren und entsprechend zu lenken, leistet einen Beitrag zur sozialen Integration (112). Die Positionen der interviewten Institutionen im Bezug auf Rand- bzw. Problemgruppen und deren Integration über forstliche Projekte und Maßnahmen variiert sehr stark. Einerseits gibt das Sozialamt zu bedenken, dass Beschäftigungsprojekte im Wald keinen Effekt haben, da zwar eine Qualifikation stattfindet, aber das anschließende Angebot eines Arbeitsplatzes nicht geboten wird (18). Der Vertreter des Forstamtes unterstreicht die Haltung des eigenen Amtes mit dem Zitat von Theodor Heuss:

„Der Wald ist die billigste Sozialeinrichtung Deutschlands“ (I10)

Diese Aussage weist darauf hin, dass sich der Forstvertreter der Verantwortung und des Effektes des Waldes für alle Personengruppen der Stadt bewusst ist. Die Institution Staatliche Schlösser und Gärten ist gerne bereit im Rahmen der Beschäftigungsförderung einen Beitrag zu leisten und hat dies in der Vergangenheit auch getan, ist aber aufgrund von fehlenden Räumlichkeiten, Betreuung und Problemen mit der vorgesetzten Dienststelle, zurzeit nicht in der Lage, diese Projekte weiterzuführen (112). Die Arbeitsförderungsgesellschaft AGIL mbH sieht in der eigenen Arbeit einen positiven Beitrag zur Beschäftigungspolitik des Landkreises („Aushängeschild des Landkreises“ 112) und eine immense Kostenersparnis, da Arbeiten abgeleistet werden, die sonst nicht verrichtet werden können oder nur mit hohem finanziellen Aufwand (112).

Grundsätzlich kann für die Projekte, Maßnahmen, Haltungen und Positionen der Institutionen in Kassel festgehalten werden, dass der Wald, wie im Fallbeispiel Göttingen, ein immenses Potential für soziale Nachhaltigkeit und soziale Integration besitzt. Aber auch hier stellt sich wiederum die Frage, ob der kommunalen Forstverwaltung die Bedeutung von sozialer Nachhaltigkeit und damit sozialer Integration bewusst ist. In fast allen Projekten der 
Fallstudie Kassel werden den Personengruppen Toleranz und Solidarität gegenübergebracht, was die Basis für eine soziale Integration legt. Die Haltung der Forstverwaltung kann aber eher als passiv bezeichnet werden, da nur das Bereitstellen von Flächen nicht als aktiver Beitrag zu werten ist. Dass die Vermittlung von Kenntnissen und Wissen, durch die Waldlehrpfade oder durch Arbeitsprojekte, Kompetenzen schafft und so zur sozialen Integration von Menschen beiträgt, ist der Forstverwaltung nicht bewusst. Das Forstamt ist auch hier zur Durchführung der Maßnahmen auf Partner aus dem sozialen Sektor angewiesen. Die Hypothese I kann für die Fallstudie Kassel verifiziert werden, denn das vordergründige Verständnis von sozialer Nachhaltigkeit und Integration lässt hier nur einen geringen Beitrag der Forstverwaltung zu, z.B. zeitliche Befristung der Arbeitsprojekte und Flächenbereitstellung durch die Forstverwaltung.

\subsubsection{Fallstudie Freiburg}

Tabelle 15: Institutionen in Freiburg i.Br., deren Maßnahmen und Partner

\begin{tabular}{|c|c|c|}
\hline \multicolumn{3}{|c|}{ Freiburg i.Br. } \\
\hline Institution & Maßnahme / Projekt & Partner \\
\hline $\begin{array}{l}\text { Suchtbeauftragter der Stadt } \\
\text { Freiburg }\end{array}$ & $\begin{array}{l}\text { - Aufenthalt von Drogenabhängigen } \\
\text { im Colombipark }\end{array}$ & \\
\hline Jugendförderung & $\begin{array}{l}\text { - Treffpunkte für Jugendliche im } \\
\text { Wald }\end{array}$ & $\begin{array}{l}\text { - Jugendsachbearbeiter der Polizei } \\
\text { - Forstamt (Revierförster } \\
\text { Mooswald) } \\
\text { - Jugendzentrum }\end{array}$ \\
\hline Forstamt & $\begin{array}{l}\text { - Wagenburgplatz im Wald } \\
\text { - Treffpunkte für Jugendliche } \\
\text { - Grillplätze } \\
\text { - Installation eines Waldseilgartens } \\
\text { - Walderlebnispfad } \\
\text { - Festveranstaltungen im Wald } \\
\text { - Beschäftigungsprojekte für } \\
\text { Jugendliche } \\
\text { - Arbeitserziehung im Wald für } \\
\text { Jugendliche, die nicht beschulbar } \\
\text { sind } \\
\text { - Wohnungslosenhilfe }\end{array}$ & $\begin{array}{l}\text { - Ämter der Baubranche } \\
\text { - s. o. } \\
\text { - Ausgleichs- und } \\
\text { Ersatzmaßnahmen (Stadtteil } \\
\text { Rieselfeld) } \\
\text { - Bürgerverein } \\
\text { - Verein zur Förderung kommunaler } \\
\text { Beschäftigung } \\
\text { - Schulen, Handwerker } \\
\text { - Rotes Kreuz, Heilsarmee, } \\
\text { Sozialamt }\end{array}$ \\
\hline Stadtrat & - Treffpunkte für Homosexuelle & \\
\hline
\end{tabular}


Die Übersicht der Maßnahmen für Problemgruppen auf Waldflächen zeigt in Freiburg ein breites Spektrum. Der Betrachtungsschwerpunkt liegt hier erneut auf den integrativen Projekten, die in Zusammenarbeit mit dem städtischen Forstamt stattfinden. In Freiburg sind im Stadtwald Grillplätze vorhanden, die von allen Personengruppen genutzt werden können. Die Koordination der Nutzung liegt beim Forstamt. Zukünftig soll hier ein Ranger eingesetzt werden, der die Grillstellen und Hütten auch spät am Abend noch kontrolliert und bei störendem Verhalten steuernd eingreifen kann. In diese Kategorie fallen auch die vom Forstamt veranstalteten Feste, so genannte "Hocks“, die vornehmlich in Stadtteilen stattfinden, wo die Identifikation der BürgerInnen mit dem angrenzenden Wald nicht so stark behaftet ist (I15).

Im Zuge des Stadtteilneubaus ,Rieselfeld' finden in Zusammenarbeit des Forstamtes mit anderen Institutionen mehrere Projekte statt, die im Folgenden näher erläutert werden sollen. Auf der Fläche des jetzigen Stadtteils war eine Wagenburgsiedlung ansässig, welche durch die Umwandlung weichen musste. Die Frage nach dem Ausgleich für die Bewohner der Wagenburgsiedlung beantworteten das Forstamt und das Bauamt der Stadt. Die Überlegungen, die Wagenburgler nicht einfach zu vertreiben bzw. innen die Wohnfläche zu nehmen, sondern ihnen einen Ausweichplatz zu bieten, zeigen, dass die Ämter dem Lebensstil der Bewohner tolerieren. Nach einigen Vorschlägen seitens des Forstamtes wird in Absprache mit dem Bauamt ein Platz ausgewiesen, auf den die Wagenburgler ausweichen können (115). Da dem Neubau des Stadtteils ,Rieselfeld' Wald zum Opfer gefallen ist, konnte im Zuge der vorgeschriebenen Ausgleichs- und Ersatzmaßnahmen auf einer entsprechenden Fläche ein Waldseilgarten angelegt werden (I15). Dieser wird von einem gemeinnützigen Verein betrieben und hat therapeutische und sozialpädagogische Ansätze zum Ziel, wie Stärkung der Teamfähigkeit, soziale Kompetenzen etc. (I15). Die Nutzungskonditionen für spezielle Nutzergruppen, z.B. Sonderschulen oder soziale Einrichtungen für Schwererziehbare aus dem Stadtbereich sind günstiger als die andrer Nutzergruppen (gestaffelte Preise) (115). Angrenzend an den Seilgarten wurde ein Walderlebnispfad angelegt, der auch die benachbarte Freifläche mit einbezieht. Da dieser Pfad von allen Bevölkerungsschichten gleichermaßen genutzt werden kann und Kenntnisse bzw. Wissen vermittelt, kann dieser zur Identifikation der NutzerInnen mit dem Wald beitragen.

Ein weiteres Projekt stellt die Schaffung von Treffpunkten für Jugendliche im Wald dar. In einem Stadtteil, der an den Mooswald angrenzt, tritt folgendes Problem auf. Jugendliche versammeln sich an ungeeigneten Plätzen, wie Tiefgaragen, Einkaufzentren und öffentlichen Plätzen, wodurch sich die umliegenden Anwohner massiv gestört fühlen (I14). Der Jugendsachbearbeiter der Polizei, der zuständige Förster des Mooswaldes, das Jugendzentrum des Stadtteils und das Amt für Jugendförderung (Sozial- und Jugendamt) 
treten daraufhin zur Problemlösung zusammen. Mit den problematischen Jugendlichen hat man eine Lösung in der Installierung von vier Treffpunkten im Wald gefunden, wo sie sich unter Einhaltung gemeinsam vereinbarter Regeln aufhalten können (I14, I15). Die Jugendcliquen dürfen diese Treffpunkte selbst einrichten und bekommen Unterstützung in Form eines Bauwagens von der Bevölkerung des Stadtteils. Durch das Angebot von Treffpunktalternativen zeigen sich die beteiligten Institutionen solidarisch mit den Jugendlichen und tolerieren den Wunsch nach Zusammenkünften mit der Möglichkeit Musik zu hören, zu rauchen und zu grillen (was in den örtlich Jungentreffs nicht möglich ist; 114).

Die Beschäftigungsprojekte, die vom Forstamt mitgetragen werden, stellen einen weiteren integrativen Bereich dar. In Freiburg gibt es zum einen den ,Verein zur Förderung kommunaler Beschäftigung', der die Qualifizierung von arbeitslosen Jugendlichen zum Ziel hat. Die Arbeiten des Vereins mit den Jugendlichen finden z. T. auf den Flächen des Forstamtes statt. Die Arbeiten sind im Winter von der Brennholzaufbereitung und im Sommer von der Sammlung von Müll geprägt (115). Bei diesen Arbeiten werden die arbeitslosen Jugendlichen zunächst wieder an einen geregelten Tages-, Arbeits- und Lebensrhythmus gewöhnt. Weiterhin qualifizieren sich die Jugendlichen durch den Umgang mit Motorsägen und anderen technischen Gräten und haben somit eine erhöhte Chance, auf dem ersten Arbeitsmarkt eine Beschäftigung zu finden (I15). Die Vermittlung in den Arbeitsmarkt und die Verminderung der Jugendarbeitslosigkeit haben bei den Beschäftigungsprojekten oberste Priorität. Zudem werden die Flächen des Forstamtes genutzt, um nicht beschulbare junge Menschen zu beschäftigen. Hierbei handelt es sich um ein neues Projekt, in dem verhaltensauffällige Jugendliche, die z.B. hyperaktiv sind und nicht im Klassenverband lernen können, mit einem Arbeitserzieher Arbeiten im Wald erledigen (I15). Ziel ist, die Kompetenzen der jungen Menschen zu stärken und z. B. der Hyperaktivität entgegenzuwirken.

Gerade in Großstädten häuft sich die Zahl der Wohnungslosen und es ist ein weit verbreitetes Phänomen, dass sich diese Menschen in den Wald zurückziehen. Das Forstamt äußert hier, dass Einzelsituationen von Personen, die im Wald vornehmlich schlafen, vorübergehend geduldet werden. Hierzu besteht eine Zusammenarbeit des Forstamtes mit dem Roten Kreuz bzw. der Heilsarmee. Die karitativen Einrichtungen haben bisweilen mit Menschen zu tun, denen es aufgrund psychischer Störungen unmöglich ist, in Wohnungen zu leben. Hier werden dann in Absprache mit dem Forstamt Lösungen im Wald gefunden, wie z.B. einen Wohnwagen für diese Person an einem bestimmten Platz abzustellen. Diese Lösungen sind zeitlich begrenzt und diejenigen Personen müssen sich an abgesprochene Regeln halten.

Die Dauer der oben aufgeführten Maßnahmen und Projekte ist unterschiedlich. Die Beschäftigungsprojekte sind meist auf die Dauer von einem halben bis zu einem Jahr 
angelegt um überhaupt Effekte in der Qualifizierung und Vermittlung zu erzielen. Der Ausweichplatz für Wagenburg ist auch auf einen längeren Zeitraum ausgelegt, da sich die Menschen auf ein Leben dort einrichten. Im Falle des Waldseilgartens und des Walderlebnispfads handelt es sich um permanente Einrichtungen, die auf lange Sicht Erfolge zeigen. Bei der Tolerierung von Wohnungslosen im Wald ist jeweils eine zeitliche Begrenzung vereinbart, da hier der Anspruch besteht, diese Menschen in Wohnungen zu vermitteln um deren Gesamtsituation positiv verändern zu können. Bleibt hier die Frage offen, ob die komplexen Probleme dieser Personen durch die Unterbringung in einer Wohnung beseitigt werden können. Die Installation der vier Treffpunkte für Jugendliche im Wald ist auf Langfristigkeit ausgelegt und in den Interviews wird ein jährlicher Bedarf immer wieder unterstrichen (114). Allerdings sind von den ehemals vier Treffpunkten nur noch zwei vorhanden, da rivalisierende Jugendcliquen die Treffpunkte der jeweils anderen zerstört haben und sie sich z. T. nicht an die vereinbarten Regeln gehalten haben.

Den o. g. Projekten werden von den einzelnen Institutionen unterschiedliche integrative Erfolge zugeschrieben. So wird in der Treffpunktgestaltung für Jugendliche die Minimierung der Konflikte im Stadtteil, eine Reduzierung der Anzeigen wegen Ruhestörung und Belästigung und die Verbesserung des sozialen Gefüges der Jugendlichen als integrativer Erfolg betrachtet (114). Wobei hier die Jugendförderung zu bedenken gibt, dass zunächst in der Arbeit das Verantwortungsbewusstsein und die Selbstorganisation der Jugendcliquen überschätzt werden. Es werden z.B. Absprachen über die Nutzung mit Cliquen besprochen, die wenig später nicht mehr in der gleichen Zusammensetzung der Jugendlichen bestehen. Es wurde von einer Unterschätzung der Veränderungen bzw. Fluktuation von Mitgliedern innerhalb dieser Cliquen gesprochen (115). Diese Tatsachen erschweren einen Erfolg dieser Maßnahmen, dennoch wird die Aufrechterhaltung zweier Treffpunkte als Erfolg gewertet. Von einer erfolgreichen Vermittlung in den Arbeitsmarkt durch die Beschäftigungsmaßnahmen konnte nicht berichtet werden. Es wurde von Seiten des Forstamtes von Versuchen zur Übernahme Jugendlicher in Ausbildungsverträge berichtet, die aber nach einiger Zeit an den Lebensumständen der Personen scheiterten, Bsp. Rückfall in die Drogenabhängigkeit (I15).

Die Äußerungen der einzelnen Institutionen zu den Randgruppen und deren Aufenthalt im Wald bzw. auf Grünflächen tragen ebenfalls einen Teil zur Integration bei. Auffällig sind in diesem Fallbeispiel die Meinungen des Forstamtes zu den Gruppierungen. Die liberale Einstellung gegenüber Wohnungslosen, die im Wald Unterschlupf suchen, äußert sich wie folgt:

„Weil wir denken, Wald im Verdichtungsraum und Wald in der Gesellschaft heute, in der Gesellschaft, in der es immer mehr Randgruppen gibt, in der das soziale Gefälle immer mehr auseinander driftet, hat diese Funktion Lebensraum anzubieten für Menschen, die in Not gekommen sind." (I15) 
„Das ist aus meiner Sicht für alle Beteiligten immer noch günstiger, es leben Leute in verträglicher Weise im Wald als unter der Brücke, in der Stadt, an der Dreisam." (I15)

Diese Aufgabensicht macht deutlich, dass das Potential zur Integration von sozialen Randgruppen erkannt ist und aktiv verfolgt wird. Auch der folgende Auszug belegt die Einstellung des Amtes zum Potential des Waldes:

„Wenn Jugendliche im Stadtteil keine solchen Freiräume finden, wenn sie überall stören,
ja dann müssen wir sie halt in Gottes Namen (...) Dann müssen wir sie woanders bieten.
Dann kann Wald per se nicht sakro sankt sein für solche Dinge, sondern dann müssen
wir gucken, wo ist es verträglich, wo machen wir so was, so war das auch mit der
Wagenburg." (I15)

Ein Erkennen der sozialen Problematik und ein aktiver Beitrag zur Lösung kann an dieser Textstelle abgelesen werden. Die Haltung des Forstamtes zu den sozialen Problemen wird durch eine starke Einbindung in die Gesamtinteressen der Stadt unterstützt. Zudem sieht der Vertreter des Amtes es als unerlässlich an, sich an einem offenen Informationsaustausch und Diskussionen über Problemlagen zu beteiligen. Vor allem die aktive Mitgestaltung dieser Diskussion kommt dem integrativen Potential des Waldes entgegen:

\begin{abstract}
„Ich denke, der stadtnahe Wald hat mehr denn je solche Aufgaben im klassischen Bereich Park, Wald, Sportfläche, Kulisse für Freizeit und Erholung, aber auch für solche sozialen Randgruppen, die können dort unterkommen und ich denke, noch mehr als das heute der Fall ist, wenn man so die gesellschaftliche Entwicklung sieht, aber das war ein langer Prozess, da muss man sich auch erst einfinden und die Diskussion auch ein bisschen mitgestalten." (I15)
\end{abstract}

Diese Einstellung zeigt, dass eine Nutzung des Waldes durch Randgruppen toleriert wird. Die allgemeine Position des Forstamtes legen die Textbeispiele offen und weiterhin wird deutlich, dass das Amt in Konfliktfällen an Einigungen mit den Gruppierungen und nicht an Sanktionen interessiert ist (115). Die Positionen des Forstamtes lassen sich auf das selbst erstellte Leitbild zurückführen, worin die Ziele und Produkte zu finden sind, die das Amt erreichen und anbieten will (115). Hierin findet sich auch die Zusammenarbeit mit dem „Verein zur kommunalen Beschäftigungsförderung" als ausgewiesenes Ziel wieder (Burgbacher, 2001). Diese Leitbilderarbeitung hat eine deutliche Ausrichtung auf ein Dienstleistungsunternehmen, in dem Erwartungshaltungen der Bevölkerung und die Vorschläge der eigenen Mitarbeiter in Einklang gebracht werden. Um sich in die Problemlagen der Stadt einbringen zu können, müssen aktiv Kontakte zu den unterschiedlichen Gruppierungen (z.B. Jagd, Naturschutz) und kommunalen Institutionen gehalten werden (115). Der Vertreter des Forstamtes macht weiterhin deutlich, dass gesellschaftliche Probleme nur gemeinsam mit anderen Disziplinen gelöst werden können und der Forst in der Lage ist, einfache, schnelle und kostengünstige Lösungen anzubieten (I15). Als Beispiel einer Position des Konfliktlösers kann die Suche nach einem Standort für die Wagenburg gesehen werden, denn hier hat das Forstamt aktiv Vorschläge im Wald 
angeboten, die dann baurechtlich geklärt werden mussten und so eine Lösung des Problem herbeigeführt haben.

Die Fallstudie Freiburg zeigt durch die Vielzahl von Projekten ein immenses integratives Potential des Waldes auf. Somit ist der erste Teil der Hypothese I zu bestätigen. Die unterschiedlichen Projekte weisen alle eine Solidarisierung und Tolerierung von Problemund Randgruppen auf und sind zunächst als geringer Beitrag zur sozialen Integration durch die Forstverwaltung zu sehen. Auffällig sind hierbei aber die Position und die Haltung des Forstamtes gegenüber diesen Projekten. Es wird bisher in keinem Beispiel eine derartig aktive Mitgestaltung der Projekte aufgedeckt. Hier scheint das integrative Potential des Waldes seitens des Forstamtes deutlich erkannt und genutzt zu sein. Dies lässt auf die Bewusstmachung der Bedeutung von sozialer Nachhaltigkeit im Wald gerade in Großstadtnähe schließen. Dies wird vor allem durch die Maßnahmen zur Identifikation der BürgerInnen (Feste, Grillhütten) deutlich, wobei die Sozialintegration von allen gesellschaftlichen Gruppen positiv beeinflusst wird. Die Projekte zur Arbeitsförderung sind vor allem auf die Qualifizierung ausgerichtet. Die Vermittlung von Kenntnissen und Wissen kann zu einer Plazierung der Personen auf dem Arbeitsmarkt beitragen und somit deren soziale Positionierung verbessern. Bemerkenswert an dieser Stelle ist die Aufnahme der Kooperation mit einem gemeinnützigen Verein zur Beschäftigungsförderung in das Leitbild der Forstverwaltung. Dies weist zum einen auf die gesellschaftliche Verantwortung der Forstverwaltung hin. Zum anderen kann leider noch kein nachhaltiger Erfolg der Beschäftigungsmaßnahme nachgewiesen werden (Arbeitsplatzvermittlung). Dennoch sind die eigenen Aktivitäten des Forstamtes hier herauszustellen, die eine Orientierung an einem neuen Aufgabenverständnis andeuten. Der zweite Teil der Hypothese I (geringer Beitrag der Forstverwaltung zur Erschließung des integrativen Potentials) kann demnach für die Fallstudie Freiburg nicht bestätigt werden. Die Forstverwaltung hat die eigene Aufgabe als Löser von sozialen Problemen erkannt und arbeitet bewusst an diesen Integrationsprojekten mit. 
5.3.4 Fallstudie Frankfurt a.M.

Tabelle 16: Institutionen in Frankfurt a. M., deren Maßnahmen und Partner

\begin{tabular}{|c|c|c|}
\hline \multicolumn{3}{|c|}{ Franfurt a. M. } \\
\hline Institution & Maßnahme / Projekt & Partner \\
\hline Jugend- und Sozialamt & $\begin{array}{l}\text { - integrative Ferienspiele sog. } \\
\text { „Ferienkarussell“ }\end{array}$ & $\begin{array}{l}\text { - CeBeeF (Club Behinderter und } \\
\text { ihrer Freunde e.V), Lebenshilfe } \\
\text { Frankfurt, Naturschule Hessen }\end{array}$ \\
\hline $\begin{array}{l}\text { CeBeeF e.V. (Club Behinderter und } \\
\text { ihrer Freunde e.V.) }\end{array}$ & $\begin{array}{l}\text { - Freizeitangebote für Menschen } \\
\text { mit Behinderungen }\end{array}$ & $\begin{array}{l}\text { - Jugend- und Sozialamt, } \\
\text { Umweltamt, Naturschule Hessen }\end{array}$ \\
\hline Werkstatt Frankfurt e.V. & $\begin{array}{l}\text { - Beschäftigungsprojekte für } \\
\text { Langzeitarbeitslose }\end{array}$ & $\begin{array}{l}\text { - Agentur für Arbeit, Sozialamt, } \\
\text { Ämter der Stadt (Grünfächenamt, } \\
\text { Umweltamt, Sport- und Badeamt), } \\
\text { Kindergärten, Schulen }\end{array}$ \\
\hline Stadtgrün Forst & $\begin{array}{l}\text { - Beschäftigungsprojekte für } \\
\text { Langzeitarbeitslose (vor } 15 \text { Jahren) } \\
\text { - Beschäftigungsprojekte für } \\
\text { straffällige Jugendliche } \\
\text { - Behindertengerechte Spielplätze }\end{array}$ & $\begin{array}{l}\text { - Sozialamt (Sozialarbeiter) } \\
\text { - Jugendgerichtshilfe } \\
\text { - Behindertenverein, Elternverein } \\
\text { behinderter Kinder }\end{array}$ \\
\hline
\end{tabular}

Die Tabelle zeigt die Projekte und Maßnahmen, die sich während der Untersuchungsphase in Frankfurt aufdecken ließen. Der Kontakt zum Jugend- und Sozialamt wurde über die Ferienspiele geknüpft, da hier integrative Maßnahmen stattfinden. Bei den Ferienspielen handelt es sich vornehmlich um Freizeitveranstaltungen für Kinder in den Sommerferien (118). Unter dem Motto „Miteinander leben und erleben“ treffen sich Kinder mit und ohne Behinderungen über den Zeitraum von einer Woche und unternehmen täglich verschiedene Aktivitäten, wie z.B. der Besuch eines Bauernhofes oder eines Spielplatzes (118). Zudem finden Reiterfreizeiten statt, die ausschließlich auf behinderte und nicht behinderte Mädchen ausgerichtet sind. Hierbei fahren die Mädchen eine Woche auf einen Reiterhof und erleben die Gegebenheiten, von Stallarbeit bis hin zum Reitunterricht, gemeinsam (118). Ziel dieser Aktionen ist die Integration von Menschen mit Behinderungen in die Gesellschaft, d.h. die Annerkennung der Menschen mit ihren Einschränkungen und der unbeschwerte Umgang mit innen. Mit dem gleichen Ziel und der Unterstützung der Teamfähigkeit der Teilnehmer wurde eine Floßfahrt in Zusammenarbeit mit dem CeBeeF e.V. ins Leben gerufen. Hier haben behinderte und nicht behinderte Kinder zunächst ein Floß gebaut und sind damit auf der Nidda, einem nahe gelegen Fluss, gefahren (I18, I19). Bei dieser Aktion sind noch weitere Institutionen beteiligt, wie das Umweltamt und die Naturschule Hessen, wobei das Umweltamt den Kontakt zur Naturschule herstellt und diese wiederum für die Durchführung zuständig ist (I19). Der Leitsatz dieser Aktion "Gemeinsam gehen wir nicht unter" macht deutlich, worauf es bei der Interaktion der Menschen mit unterschiedlichen Einschränkungen 
ankommt, nämlich gegenseitiges Vertrauen, sich aufeinander verlasen können und dass mit der Hilfe Anderer das Ziel besser erreicht wird (I19). Die Aktivitäten umfassen eine Dauer von einem Tag bis zu einer Woche. Hierbei können kurzzeitige Effekte im Verhalten der Teilnehmer erreicht werden (I18, I19). Bei den o. g. Projekten konnte keine Beteiligung des Forstsektors festgestellt werden, obwohl Maßnahmen mit der Klientel behinderte und nicht behinderte Kinder und Jugendliche im Wald vorstellbar wären und dieses Potential bis dato ungenutzt bleibt.

Die Werkstatt Frankfurt, als eingetragener Verein, fungiert als Beschäftigungsträger für Langzeitarbeitslose. Der Verein organisiert und akquiriert Arbeit aus verschiedenen Bereichen für diese Klientel. Das Ziel der Projekte ist die qualifizierende Arbeit, an deren Ende ein Zertifikat stehen soll, welches die Personen in eine verbesserte Bewerbungssituation auf dem Arbeitsmarkt versetzen soll. Das Sozialamt und die Agentur für Arbeit versorgt die Einrichtung mit Klienten, die dann in die einzelnen Bereiche der Einrichtung eingewiesen werden. Vornehmlich finden die Beschäftigungsmaßnahmen im Garten- und Landschaftsbau statt, wie z.B. Begrünung von Parkplätzen, Instandsetzen von Wegen (I20). Hierbei treten einzelne Ämter der Stadt als Auftraggeber auf, vor allem das Grünflächenamt. Einen weiteren Bereich der Beschäftigungsprojekte stellt die Brennholzaufarbeitung dar. Das Brennholz fällt bei eigenen Tätigkeiten an oder wird von Firmen an die Einrichtung geliefert, wo es aufbereitet und verkauft wird (I20). Bei den Maßnahmenteilnehmern handelt es sich zu 90\% um ungelernte Kräfte (I20), die über neun Monate, mit der Option einer Verlängerung von drei Monaten, in der Einrichtung arbeiten mit dem Ziel der Qualifizierung. Als integrativer Erfolg wird die Vermittlung der Klientel in eine weitere Maßnahme, die Heranführung an einen geregelten Tagesablauf, die Bekämpfung anderer Probleme, wie z.B. Alkoholsucht oder psychischer Probleme oder die Vermittlung von „Helferjobs“ in Zeitarbeitsfirmen oder in Gartenbaubetrieben angesehen (I20).

Der Fachbereich Stadtgrün, als Abteilung des Grünflächenamtes, nutzt das integrative Potential des Waldes durch folgende Projekte. Zum einem bestand vor 15 Jahren (I21) ein Beschäftigungsprojekt für Langzeitarbeitslose in Zusammenarbeit mit dem Sozialamt in Frankfurt. Bei diesen Arbeitsprojekten stand die Wiedereingliederung in das soziale Umfeld und das Berufsfeld der Personen im Vordergrund (121). Als integrativer Erfolg der Projekte ist die Einstellung eines Maßnahmenteilnehmers im Fachbereich Stadtgrün zu nennen (I21). Diese Person hat eine Beschäftigungsmaßnahme durchlaufen, sich durch Eigeninitiative und großes Engagement hervorgehoben und bekam die Möglichkeit, sich in zwei weiteren Jahren im Fachbereich zu bewähren, was in einer festen Anstellung mündete (I21).

Als weiteres Projekt, was das integrative Potential des Waldes nutzen könnte, ist eine Vereinbarung mit der Jugendgerichtshilfe und dem Fachbereich Stadtgrün zu sehen. Die Vereinbarung besteht in der Beschäftigung von straffällig gewordenen Jugendlichen. In 
diesen Projekten sollen Jugendliche, die eine Strafe in Form von Arbeitsstunden bzw. Sozialstunden ableisten müssen, eine Beschäftigung in den Revieren des Fachbereiches finden. Diese Einsätze von Jugendlichen sind mit den verantwortlichen Revierbeamten im Vorfeld abgesprochen worden (I21). Im Interview verweist der Gesprächspartner auf die Vereinbarung zwischen den beiden Institutionen, da eine solche Maßnahme bis zu diesem Zeitpunkt noch nicht stattgefunden hat. D.h. der Fachbereich Stadtgrün steht auf Anfrage bereit und könnte eine Beschäftigungsmaßnahme durchführen. Auf eine Besonderheit soll hier kurz eingegangen werden, denn exakt zum Zeitpunkt des Interviews kontaktiert die Jugendgerichtshilfe den Interviewpartner bezüglich der Beschäftigung eines 19-jährigen, der eine Strafe in Form von Arbeitsstunden abzuleisten hat. Die betreffende Person sollte sich wegen organisatorischer Fragen direkt an einen zuständigen Revierleiter wenden und den Arbeitseinsatz besprechen. Auf Nachfrage via E-Mail der Verfasserin am Fachbereich Stadtgrün, ob diese Maßnahme erfolgreich stattgefunden hat und ob aus diesem Projekt weitere Projekte mit der Jugendgerichtshilfe entstanden seien, folgte folgende Antwort:

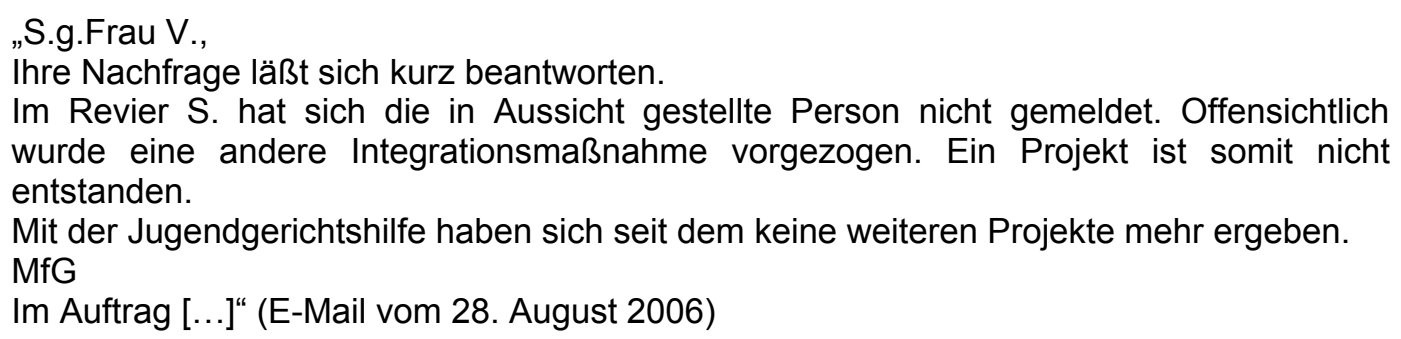

Es bleibt festzuhalten, dass die Bereitschaft des Fachbereiches Stadtgrün einen Beitrag zur Integration von Problemgruppen leisten zu wollen, grundsätzlich besteht. Dies Angebot bzw. das Potential der Beschäftigung im Wald wird dennoch von Seiten der Jugendgerichtshilfe bis zu diesem Zeitpunkt nicht genutzt.

Des Weiteren hat der Fachbereich Stadtgrün die Einrichtung von behindertengerechten Spielplätzen mitgetragen. Hier fungieren als Partner ein Behindertenverein, zu dem keine näheren Angaben gemacht werden, und engagierte Eltern von Kindern mit Behinderungen. Die Spielparke sind so konzipiert, dass Kinder mit und ohne Behinderungen dort spielen und sich bewegen können. Die einzelnen Spielgeräte können mit Rollstühlen über Rampen befahren werden und viele akustische Elemente, wie z.B. die „Schiffsverständigung“ legen die Grundlage für ein Zusammenspiel von Kindern mit und ohne Behinderungen (I21). Diese Einrichtungen sind dauerhaft angelegt und weisen eine intensive Nutzung gerade in den Sommermonaten auf. Im Interview wurde die Tatsache betont, dass die Gestaltung solcher Spielparke dem Forst oblag und durch die Zusammenlegung mit dem Grünflächenamt die Anerkennung für diese Projekte an einen anderen Fachbereich verliehen wird (I21). Das Ziel dieser Projekte ist, Kinder mit unterschiedlichen gesundheitlichen Hintergründen zusammenzuführen und deren Zusammenleben zu normalisieren. 
Zudem geben die allgemeinen Äußerungen der Interviewpartner Auskunft über die Tolerierung der verschiedenen Personengruppen. Diese sprachlichen Äußerungen sind als Handlungen zu verstehen, die den Ausdruck eigener Wertungen darstellen (Fuchs, 1999, S. 170) Im Gespräch mit dem Forstamt konnten sowohl wohlwollende als auch kritische Aussagen zu den Personengruppen aufgedeckt werden. Auf der einen Seite wird das Verhalten der Personen, die an einer Arbeitsmaßnahme im Fachbereich Stadtgrün teilnehmen, kritisiert.

„....weil wenn einer die Forstarbeit Herbst, Winter, Frühling nicht gewöhnt ist, die kommen einfach nicht, die sind am dritten Tag krank und nicht nur einen Tag, sondern dauerhaft." (I21)

„Der sagt ihnen, ich habe schon 100 Jahre gepinselt, gestrichen, ich habe schon Steine geschleppt und da soll ich jetzt zu euch kommen. Wenn ich so einen Mann schon sehe, der von der Sprache schon so lethargisch ist, wenn man meint, er hätte eine Schlaftablette geschluckt,..." (121)

Die Unzuverlässigkeit und Lethargie der Maßnahmenteilnehmer stehen bei der Kritik im Vordergrund, so dass es den fachbereichseigenen Mitarbeiter kaum zuzumuten ist, diese Problemklientel in die tägliche Arbeit einzugliedern. Auch die Gefährlichkeit der Waldarbeit ließe eine Eingliederung von z.B. Suchtkranken nicht zu (I21). Dennoch wird die Stärkung des sozialen Umfeldes durch Heranführung an Arbeit als äußerst positiv bewertet.

„Ich finde es ja nicht schlecht, dass solche Leute wieder herangeführt werden und es ist besser, sie arbeiten gering als gar nicht und...ja, stärken unter Umständen auch das Sozialfeld wieder." (I21)

Diese Beispiele sollen die Ambivalenz der Meinung der Personen, die sich verantwortlich für die Projekte zeichnen, deutlich machen.

Ähnlich verhält es sich mit den Äußerungen gegenüber Obdachlosen Personen im Wald. Einerseits wird versucht sie aus ihren Refugien im Wald zu vertreiben. Anderseits wird hingenommen, dass sich die Personengruppe nach der Aufforderung den Platz im Wald zu verlassen, eine neue „Nische“ suchen.

„Wenn die den Ort verlassen und ich bin oder ein Kollege dann, was weiß ich, am nächsten Tag wieder da und der ist weg, dann ist ja in Ordnung, wenn der drei Abteilungen weiter gezogen ist, muss ich inn erst mal wieder finden und das ist Methode Zufall, ich habe nicht Zeit, Leute zu suchen." (I21)

„....ich kann nicht täglich kontrollieren, wo der sich hingezogen hat. Der kann wirklich wenige $100 \mathrm{~m}$ sich versetzen und ich finde den erst wieder nach drei Jahren, dann hat er ja das was er braucht letzten Endes." (I21)

Auch diese Äußerungen können als Ausdruck eigener Wertungen verstanden werden, nämlich als Tolerierung der Anderen in der Andersartigkeit, womit ein Beitrag zur sozialen Integration geleistet wird. Eine Äußerung, die eher missbilligenden Charakter besitzt und Personen, die an Beschäftigungsprojekten teilnehmen, negativ bewertet, ist der Verdacht des Diebstahls.

„Es gibt aber auch Unterkünfte, die abseits liegen und da hatten wir just immer so zwei, drei Monate nach den Beschäftigungen, dicke Einbrüche. Dass wir also hohe 
Materialverluste und Materialwertschäden hatten, die in die tausende gingen, wo wir gesagt haben, wir können die nicht mehr nehmen, die kundschaften uns aus, die gehen weg und kommen nachts wieder." (I21)

Aussagen dieser Art leisten, der Definition entsprechend, keinen Beitrag zur sozialen Integration, da sie eine negative Wertung gegenüber den Personengruppen ausdrücken. In diesem Gespräch lassen sich auch Aussagen finden, die den Aufenthalt von sozialen Problemgruppen im Wald generell bewerten. Hier wird ein Unterschied zwischen kriminell auffälligen Randgruppen (I21) und nicht kriminell auffälligen Randgruppen gemacht, wie z.B. Personen, die aufgrund gesundheitlicher Einschränkungen zur Randgruppe werden. Bei erstgenannter Gruppe ist der Aufenthalt im Wald, den Äußerungen zur Folge, nicht gewollt. Während für die letztgenannte Gruppe Maßnahmen initiiert wurden, die gezielt darauf ausgelegt sind, sich im Wald aufzuhalten, z.B. behindertengerechte Spielparke (I21). Gerade die Äußerungen zu einzelnen sozialen Problemgruppen machen deutlich, dass ein eingeschränktes Verständnis von sozialer Integration und Nachhaltigkeit auf Seiten der Forstverwaltung vorherrscht. Durch das eingeschränkte Verständnis können die Projekte nicht tiefgreifend auf die soziale Integration wirken. Es können zwar durch Qualifizierungsmaßnahmen und Vermittlung von Kenntnissen und Wissen die Möglichkeit zu einer neuen Plazierung von unterschiedlichen Personengruppen gegeben werden, was z. Zt. aber nicht aktiv unterstützt wird. Das Forstamt kann eine solche sozialintegrative Maßnahme aus der Vergangenheit aufweisen, bei der eine Stelle für einen Langzeitarbeitslosen geschaffen wurde. Auffällig ist bei den Aktivitäten der Forstseite, dass zum Erhebungszeitpunkt keine integrative Maßnahme stattfindet, was auf das eingeschränkte Verständnis von sozialer Nachhaltigkeit und den Aufgaben des Forstamtes hinweist. Die Bereitstellung von Spielplätzen, Wanderwegen, Erholungseinrichtungen, Informationen in unterschiedlichster Form (z.B. Tafeln, Stadtwaldhaus etc.) umfasst nur einen Teil der Sozialfunktion des Waldes und lässt z.B. die soziale Integrationsfunktion außer Acht. Hypothese I kann demnach für die Fallstudie Frankfurt bestätigt werden. 


\subsubsection{Fallstudie Hamburg}

Tabelle 17: Institutionen in Hamburg, deren Maßnahmen und Partner

\begin{tabular}{|l|l|l|}
\hline \multicolumn{2}{|c|}{ Hamburg } \\
\hline \multicolumn{1}{|c|}{ Institution } & \multicolumn{1}{|c|}{ Maßnahme / Projekt } & \multicolumn{1}{c|}{ Partner } \\
\hline $\begin{array}{l}\text { pessellschaft für Arbeit und } \\
\text { Integration } \mathrm{mbH})\end{array}$ & - Beschäftigungsprojekte für & - Umweltbehörde \\
(ehemals GATE GmbH) & - Beschäftigungsprojekte für & - Forstamt, Umweltbehörde, \\
\hline Revierförsterei des Forstamtes & Suchtkranke & Sozialamt, Agentur für Arbeit \\
Hamburg & - Beschäftigungsprojekte für & - passage gGmbH \\
& Suchtkranke & \\
& - Unweltbildung für Menschen mit & - k. A. \\
\hline
\end{tabular}

Die untersuchten Projekte zur Integration von sozialen Randgruppen in Hamburg weisen einen Schwerpunkt in Beschäftigungsprojekten auf. Als Beispiel soll hier näher auf die Zusammenarbeit einer Revierförsterei des Forstamtes Hamburg (als Abteilung der Behörde für Wirtschaft und Arbeit) und der passage $\mathrm{gGmbH}$ eingegangen werden. Das Beschäftigungsprojekt findet auf den Flächen des Forstamtes statt und wird von der passage gGmbH begleitet und betreut. Es handelt sich bei den Teilnehmern der Maßnahme vornehmlich um (ehemalige) Suchtkranke, wie Drogengefährdete, Drogenabhängige, Menschen, die Substitutionsmittel (z.B. Metadon) konsumieren. Zudem auch Alkoholabhängige jeglichen Alters und mit unterschiedlichen Hintergründen, wie z.B. einschlägige Drogenkarrieren, Absolvierung mehrerer Therapien. In den meisten Fällen geht mit der Abhängigkeitserkrankung auch die Abhängigkeit von Sozialhilfe einher. Diese Klientel wird in der Revierförsterei zum Entfernen der Spätblühenden Traubenkirsche eingesetzt, wobei es sich hier um eine körperlich schwere Arbeit handelt, die aber von ungelernten Kräften geleistet werden kann. Diese Beschäftigungsmaßnahmen sind längerfristig ausgerichtet, da sie auf ein ganzes Jahr angelegt sind und insgesamt drei Jahre in dem beschriebenen Rahmen stattfinden. Die Vorgabe des Forstamtes war die Bearbeitung von ca. 80 bis 100 ha der Revierförsterei, was auch in den ersten zwei Jahren geleistet wurde (I 23). Neben der Bekämpfung der Spätblühenden Traubenkirsche wurden die Maßnahmenteilnehmer auch für das sog. Heidekusseln (I22; 123) eingesetzt (das Entfernen von jungen Bäumen aus der Heidelandschaft). Diese Tätigkeit findet auf den Heideflächen der Revierförsterei statt und wird als "sehr arbeitsintensive, händische Arbeit" (I23) beschrieben, für die eigene Fachkräfte überqualifiziert sind. Die integrativen Ziele der beschriebenen Projekte sind zum einen, den Teilnehmern durch die Beschäftigung eine Tagesstruktur zu geben um Rückfalle in die Abhängigkeit zu minimieren (I22). Zum anderen soll versucht werden, die Menschen in Arbeitsverhältnisse zu vermitteln, wobei hier nicht 
unbedingt auf den ersten Arbeitsmarkt abgezielt wird, sondern z.B. die Unterbringung bei einer Zeitarbeitsfirma (122). Laut passage $\mathrm{gGmbH}$ ist mit einer Vermittlungsrate von $10 \%$ das Ziel erreicht. Im Vordergrund steht demnach die Stabilisierung des Umfeldes der Maßnahmenteilnehmer, wie die Klärung der Wohnsituation und offener Justizfragen oder die Hilfe einer Schuldnerberatung anzunehmen.

Auch hier sollten die getätigten Äußerungen der Gesprächspartner in die Betrachtung der sozialen Integration einbezogen werden. So wird von Seiten des Forstamtes Bedenken geäußert, dass sich gewisse Randgruppen, wie Obdachlose, wieder in die Gesellschaft integrieren lassen, wenn sie einmal ganz am Rande standen (123). Grundsätzlich wird der Aufenthalt von Problemgruppen im Wald positiv bewertet. Gerade für Kinder und Jugendliche, sei dies sehr wichtig, aber im Zuge der Mediatisierung schwer zu realisieren (123). Da die dauerhafte Einrichtung von Menschen im Wald als Wohnraum gesetzlich nicht gestattet ist, muss als Forstbeamter dagegen vorgegangen werden. Dennoch berühren die Menschen und ihre Geschichte und es wird versucht, einen Weg zur Hilfe zu finden.

„Ich kann es ja nicht tolerieren, dass sie da dauerhaft leben oder auf Dauer einrichten. Auf der anderen Seite will ich innen das Letzte, was sie haben noch wegnehmen und sagen, so jetzt verschwinde hier (...), das würde ich nie tun, das wäre völlig unsozial, da muss man auch irgendwo versuchen einen Weg zu finden." (I23)

„Und das ist schon manchmal bitter, wenn ich auch im Winter auszeichne und dann schneebedeckt so einen Hügel sehe und dann merke ich, das ist jemand, der im Schlafsack im Freien da schläft, im Winter, dann tun mir diese Leute richtig leid." (I23)

Diese Aussagen weisen Verständnis und Toleranz der unterschiedlichen Lebenssituationen der Menschen auf und können somit als Beitrag zur sozialen Integration angesehen werden. Die allgemeinen Haltungen der beteiligten Ämter tragen ebenfalls zur Integration von Randgruppen bei. So sieht sich die passage $\mathrm{gGmbH}$ unter einem gewissen Vermittlungsdruck durch die Wirtschaftsbehörde und versucht dies über die human geregelten Beschäftigungsmöglichkeiten zu erreichen (I22). Als wichtigste Aussage ist hier festzuhalten, dass es möglich ist, diese schwierige Klientel überhaupt zu beschäftigen, was mit hohem Aufwand (Zeit, Werkzeug etc.) verbunden ist, aber zur Anerkennung und Selbstbestätigung der Maßnahmenteilnehmer führt. Die Haltung des Forstamtes ist durch einige Bedenken gekennzeichnet. Es werden Schwierigkeiten in der Maßnahmengestaltung für Jugendliche gesehen, da die positive Einstellung dieser zum Wald angezweifelt wird. Außerdem muss bei den Maßnahmen aus forstlicher Sicht auch die Abstimmung mit dem Berufsfeld der eigenen Mitarbeiter beachtet werden, da es hier keine Einschränkungen oder Beschneidungen geben darf. Das Forstamt bzw. die Revierförsterei wäre hier zur Durchführung weiterer Maßnahmen bereit, wenn die Rahmenbedingungen geweitet und eine annehmbare Leistungsbasis geschaffen würde (I23). Diese Haltungen zeigen deutlich, dass die Institutionen bereit sind, einen Beitrag zur sozialen Integration zu leisten, aber auch Bedenken äußern und Änderungen wünschen. Z.B. wird die finanzielle Situation der 
Maßnahmenteilnehmer von der sozialen Institution als relativ gut eingeschätzt, da durch die staatliche Unterstützung und die zusätzlich Beschäftigung einen Nettoverdienst von 14 EURO pro Stunde berechnet wurde (Grundlage: verheiratete Person, zwei Kinder, ALG II Empfänger; 122). Auch das Forstamt positioniert sich gegenüber den Maßnahmen und deren Teilnehmer durch kritische Äußerungen. Zum einen sollten derartige Projekte weiterhin stattfinden, aber die finanzielle Situation macht dies nicht möglich. Projekte im Wald, speziell die Bekämpfung der Traubenkirsche, zehren nach forstlicher Meinung an der Motivation der Gruppen, die dies über längere Zeit verrichten müssen. Gerade in diesem Projekt fand eine große Unterstützung durch das forstliche Personal statt, die größere Bäume, die von der Gruppe nicht beseitigt werden konnten, mit Forstspezialschleppern entfernt haben. Angemerkt wird an dieser Stelle, dass der betriebene Aufwand im Zusammenhang mit der Zertifizierung nach FSC zu sehen sei, da keine Pestizide zur Bekämpfung der Traubenkirsche eingesetzt werden dürfen (123). Um das Aufgabenfeld des eigenen Personals nicht einzuschränken und trotzdem benachteiligte Gruppen zu beschäftigen, wurde das Sammeln von Müll auf den Flächen angeregt. Dies zeigt ein Bemühen aus forstlicher Sicht, weiterhin einen Beitrag zur Beschäftigung leisten zu wollen. Dabei sollte das Tätigkeitsfeld des eigenen Personals und die Nachhaltigkeit der Kontakte zu anderen forstlichen Unternehmen nicht einschränkt werden.

Insgesamt betrachtet, wird durch die Projekte in Hamburg, die Äußerungen und Positionen der beteiligten Institutionen deutlich, dass es Potential zur Integration von sozialen Randgruppen im Wald gibt und dies genutzt wird bzw. zukünftig genutzt werden kann. Die Hypothese I kann für die Fallstudie Hamburg ebenfalls als bestätigt angesehen werden. Es finden Projekte statt, die Integration von sozialen Problemgruppen zum Ziel haben, aber dies ist nur in eingeschränkter Form zu betrachten. Das Beschäftigungsprojekt kann einen kurzen sozial nachhaltigen Effekt nach sich ziehen, aber eine Platzierung der Personen auf dem Arbeitsmarkt und damit in der Gesellschaft kann nicht nachgewiesen werden. An dieser Stelle ist zu beachten, dass das Ziel der Maßnahme nicht die Vermittlung in den ersten Arbeitsmarkt ist, sondern ggf. in den zweiten Arbeitsmarkt, auf dem auch ungelernte Kräfte die Möglichkeit zur Arbeit finden können. Die Wahl der Tätigkeit in diesem Projekt kann nicht als Qualifizierungsmaßnahme bewertet werden, denn die einfach strukturierte Arbeit ist eher als Gewöhnung an einen geregelten Tagesablauf und das (Wieder)Erlernen von Verantwortung für die Maßnahmenteilnehmer zu sehen. Dennoch kann die Vermittlung der genannten Kompetenzen die Basis für eine neue Positionierung in der Gesellschaft legen. 
5.3.6 Fallstudie Düsseldorf

Tabelle 18: Institutionen in Düsseldorf, deren Maßnahmen und Partner

\begin{tabular}{|c|c|c|}
\hline \multicolumn{3}{|c|}{ Düsseldorf } \\
\hline Institution & Maßnahme / Projekt & Partner \\
\hline $\begin{array}{l}\text { Garten-, Friedhofs- und } \\
\text { Forstamt }\end{array}$ & $\begin{array}{l}\text { - Beschäftigungsprojekte für } \\
\text { Langzeitarbeitslose: } \\
\text { 1. Servicekräfte } \\
\text { 2. Grünmobile } \\
\text { 3. Garten- u. Landschaftsbau } \\
\text { - Beschäftigungsprojekte für Menschen mit } \\
\text { Behinderungen } \\
\text { - AB Maßnahmen (ehemals) }\end{array}$ & $\begin{array}{l}\text { - ARGE Düsseldorf } \\
\text { 1. Zukunftswerkstatt Düsseldorf } \\
\text { GmbH, } \\
\text { 2. Caritas, Renatec, } \\
\text { Zukunftswerkstatt Düsseldorf } \\
\text { GmbH } \\
\text { 3. Beschäftigungsträger (keine } \\
\text { näheren Angaben) } \\
\text { - Werkstatt für angepasste Arbeit } \\
\text { GmbH }\end{array}$ \\
\hline
\end{tabular}

Die Maßnahmen zur sozialen Integration von sozialen Randgruppen beziehen sich für die Landeshauptstadt Nordrhein-Westfalens Düsseldorf auf das Garten- Friedhofs- und Forstamt. Im Gespräch wurde deutlich, dass in den vergangenen Jahren eine Vielzahl von AB-Maßnahmen stattgefunden hat. Um auf den gesellschaftlichen und arbeitsmarktpolitischen Wandel zu reagieren, hat sich die Institution zur „Schaffung von Arbeitsgelegenheiten für erwerbsfähige Bezieher/innen von Arbeitslosengeld (ALG) II“ entschlossen (Ö Vorlagen - Nr. 70/ 3/2005). Diese Maßnahmen werden in vier unterschiedliche Kategorien bezüglich des Betreuungsaufwandes eingeteilt, Maßnahmen mit 1) geringem, 2) etwas umfangreicherem, 3) starkem Anleitungs-, Betreuungs- und Qualifizierungsaufwand durch Beschäftigungsträger und 4) Maßnahmen mit Anleitungs- und Betreuungsaufwand beim Garten-, Friedhof- und Forstamt (ebd.). Deutlich wird bei dieser Auflistung die starke Einbindung von Beschäftigungsträgern in diesen Projekten. Die vier Teilbereiche der Beschäftigung bauen aufeinander auf und sind nach Zielerreichung der Teilnehmer abgestuft (124). Der erste Maßnahmentyp, der drei Tätigkeitsfelder beinhaltet, wird als „einfach" und mit geringem Aufwand charakterisiert (124). Hierbei handelt es sich um „Servicekräfte“, die in den drei Tätigkeitsfeldern Friedhöfe / Pförtner, Parks und Spielplätze und Mülltrennung auf Friedhöfen eingesetzt werden. Die Aufgaben der Servicekräfte beziehen sich auf Rundgänge auf den jeweiligen Flächen, die Information der BesucherInnen auf den Flächen und die Benachrichtigung bei Störungen, Verschmutzungen und Beschädigungen von Einrichtungen und Flächen. Die Stellen der Servicekräfte werden mit 1-Euro Kräften besetzt, die ALG II Empfänger sind und einen Euro pro Stunde Arbeitseinsatz erhalten. Die Anzahl der beschäftigten Servicekräfte variiert in den 
Tätigkeitsfeldern, so sind auf den Friedhöfen und als Pförtner 26 Personen, auf den Parks und Spielplätzen 20 Personen und zur Mülltrennung auf Friedhöfen 18 Personen eingesetzt (Ö Vorlagen - Nr. 70/ 3/2005). Ein weiterer großer Einsatzbereich für Langzeitarbeitslose mit 63 Stellen ist der zweite Maßnahmentyp, die „Grünmobile“. Hier werden zehn Kolonnen mit Fahrzeugen, Maschinen und Geräten ausgestattet, deren Hauptaufgabe die Beseitigung von Müll aus öffentlichen Grünanlagen ist. Die dritte Kategorie von Beschäftigungsmaßnahmen, die in Zusammenarbeit mit einem Beschäftigungsträger und dem Garten-, Friedhofs-, und Forstamt stattfindet, greift in den Bereich der Ausbildung (I24). In dieser Maßnahme erfolgt für 30 Personen neben einer Anleitung und Betreuung auch eine fachliche Qualifizierung für Tätigkeiten im Garten- und Landschaftsbau, z.B. Gehölzpflege, Ausbesserung von Wegen (Ö Vorlagen - Nr. 70/ 3/2005; 124). Ausdrückliches Ziel dieser Maßnahme ist eine Integration in den ersten Arbeitsmarkt nach Abschluss der Qualifikation (Ö Vorlagen - Nr. 70/ 3/2005). In der vierten Kategorie der Beschäftigungsmaßnahmen liegt der Betreuungs- und Anleitungsaufwand direkt beim Garten-, Friedhofs- und Forstamt, denn hier werden die 12 Personen von Vorarbeitern des Amtes angeleitet und für einfache manuelle Tätigkeiten auf öffentlichen Grünflächen und Friedhöfen eingesetzt (ebd.). Diese Stellen sind auf eine Dauer von 12 Monaten ausgelegt, besitzen somit langfristigen Charakter, werden nach Tariflohn bezahlt und sind sozialversicherungspflichtig (I24). Insgesamt schafft die Institution durch die Projekte in den vier Kategorien eine Beschäftigungsmöglichkeit für ca. 169 Personen, die aus der Problemgruppen der Langzeitarbeitslosen akquiriert werden. Bemerkenswert an dieser Stelle ist die Entstehung der Projekte, die direkt an die Situationen der Klientel angepasst wurden. Zusätzlich zu den o.g. Projekten vergibt das Garten-, Friedhofs- und Forstamt Aufträge der Grünflächenpflege, wie die Pflege einer Parkanlage, an die „Werkstatt für angepasste Arbeit“. Hierbei sollen Menschen mit geistigen, körperlichen und seelischen Behinderungen schrittweise und gezielt in das Arbeitsleben eingegliedert werden (I24; wfaa 2006). Eine gesellschaftliche Verantwortung des Amtes macht die Tatsache deutlich, dass diese Aufträge auch an Unternehmen vergeben werden könnten, die einen ökonomischen Vorteil für das Amt darstellen würden.

„Mit Behinderten, die kriegen dann... anstatt dass wir das an Unternehmen vergeben, geben wir das denen, ist zwar ein bisschen teurer, aber macht gesamt gesellschaftlich Sinn (...)." (I24)

Alle genannten Maßnahmen besitzen langfristigen Charakter wodurch die Chance gegeben ist, durch die Qualifikation von bis zu einem Jahr den Einstieg in den ersten Arbeitsmarkt zu schaffen. Ein Teil der Maßnahmen ist auf ein halbes Jahr begrenzt und kann bei Bedarf um weitere sechs Monate verlängert werden. Auch diese Projekte, als kurz- bzw. mittelfristig bezeichnet, zeigen Wirkung.

„(...), Ziel ist es, pro Jahr fünf zu übernehmen auf ungelernte Tätigkeiten im Garten- und Landschaftsbau." (I24) 
Das Ziel der Beschäftigungsmaßnahmen wird in dieser Aussage ganz deutlich, denn die Teilnehmer der Maßnahmen erhalten eine reelle Chance der Integration in den Arbeitsmarkt im Garten-, Friedhofs- und Forstamt. Wenn es sich hierbei auch um die Beschäftigung in einem ungelernten Beruf handelt, so ist die soziale Integrationsleistung für die Menschen als äußerst positiv zu werten. An dieser Stelle wird von dem Gesprächspartner angemerkt, dass der Aufbau der Beschäftigungsprojekte es zulässt, dass sich für die Teilnehmer, die zunächst als "Servicekraft" begonnen haben, im Verlauf der Arbeitsleistung eine Festanstellung im niedrigschwelligen Bereich (Garten- und Landschaftsbau) ergeben kann. Hier wird auf den Forstbereich verwiesen, der genau diese Möglichkeit nicht bieten kann.

„Und also auch für den Forstbereich muss man sagen, früher hatte man viel mehr Beschäftigte, die einfach mitgelaufen sind im normalen Betrieb, seit wir eine hohe Technisierung haben, geht das gar nicht." (I24)

Im Zuge der Technisierung des Forstbereiches hat der so genannte zweite Arbeitsmarkt, in dem ungelernte Kräfte einfache Arbeiten verrichten konnten, an Bedeutung verloren und bietet daher keine Möglichkeiten, ungelernte Kräfte nach kurzer Einarbeitungszeit einzusetzen.

Als integrativer Erfolg der gesamten Maßnahmen des Amtes ist die Übernahme von Personen in unbefristete Beschäftigungsverhältnisse zu werten, wenn dies auch nicht für den Forstbereich gilt.

„(...) und das sind dann auch immer welche, die wir danach mit übernehmen in unbefristete Beschäftigtenverhältnisse." (I24)

In dem erklärten Ziel des Amtes, Menschen für den ersten Arbeitsmarkt zu qualifizieren, steckt nach Aussage des Amtsvertreters das oben angesprochene Problem, dass dieser erste Arbeitsmarkt keine ausreichenden Stellen für dieses Klientel vorhält.

Neben der Schwierigkeit, die Maßnahmenteilnehmer in den Arbeitsmarkt zu integrieren, steht die persönliche Verlässlichkeit der Teilnehmer als Problem der Integration gegenüber.

„Wie gesagt, hier kommen Leute, wo ich nicht weiß, ob die morgen noch da sind, dann soll ich denen Arbeitsschuhe geben, dann soll ich denen Werkzeug geben. Also wir hatten so Fälle, die sind morgens gekommen, haben ihr Werkzeug bekommen und sind verschwunden." (I24)

Die Äußerung ist von einer Skepsis geprägt, die in gewisser Weise der Tolerierung und Akzeptanz der Anderen entgegensteht.

Die Haltung des Amtes, die durch Aussagen des Interviewpartners untermauert werden, variieren in der Bedeutung für Integration. Zum einen wird aufgezeigt, dass der Verwaltungsaufwand der Beschäftigungsmaßnahmen sehr hoch ist, Effekte ausbleiben (bis auf die Grünmobile) und ein ökonomischer Nutzen für das Amt nicht zu ersehen ist (I24). Die o.g. Servicekräfte werden in den Betrieb so integriert, dass der betriebliche Ablauf möglichst wenig gestört wird, da diese Klientel einen hohen Betreuungsaufwand erfordert. Dieser ist von den eigenen Mitarbeitern kaum zu leisten (124). Hier werden auch 
Beschäftigungsmaßnahmen angesprochen, die den Aufgabenbereich des Amtes beschneiden, wenn es sich um den Einsatz von Hilfskräften im Schulbereich handelt, die für die Grünpflege eingesetzt werden. Weiterhin wird den Maßnahmen ein niedriger Anspruch an das Ergebnis zugewiesen, da den Teilnehmer der Ablauf der Arbeiten und eine Orientierung näher gebracht werden soll. Ein ökonomischer Nutzen für das Amt ist den Beschäftigungsmaßnahmen laut Interviewpartner nicht zu entnehmen, betont wird dennoch die Möglichkeit der Tätigkeit für die Teilnehmer.

„Wie gesagt, dass lohnt sich für uns alles nicht, aber dann haben die eine Beschäftigung." (I24)

Der Gesprächspartner weist in diesem Zuge auch auf ein zukunftsfähiges Modell der Beschäftigungsförderung hin. Die Personengruppen, die einen hohen Betreuungsaufwand in der Anleitung einer Arbeit benötigen und so die Kosten in die Höhe treiben, werden auf bestimmten Flächen eingesetzt und können in den Kosten mit einer Unternehmerleistung gleichgesetzt werden. Durch dieses Modell kann Integration in den ersten Arbeitsmarkt entstehen (124). Zudem ist der Amtsvertreter der Meinung, dass die Personen die ALG II erhalten, für einen Zuschuss Arbeit leisten sollten (124). Der Haltung des Amtes durch die Äußerungen ist ein ambivalentes Verhältnis zur Integration zu entnehmen. Auf der einen Seite wird durch Akzeptanz und Solidarität des Amtes die Integration der Problemgruppen gefördert. Andererseits werden die Maßnahmen mit sehr großer Skepsis betrachtet und der Effekt deutlich in Frage gestellt, wobei es sich nicht direkt um einen Beitrag zur sozialen Integration handelt.

Die allgemeine Position des Amtes trägt ebenfalls einen Teil zur sozialen Integration bei, denn durch Äußerungen kann eine positive oder negative Haltung zur Integration abgeleitet werden. Der Gesprächspartner beschreibt zunächst die Schwierigkeiten in der Zuweisung für die Arbeitsstellen durch die ARGE in Düsseldorf. Dies zeigt, dass das Garten-, Fiedhofs- und Forstamt einen Beitrag zur Beschäftigung von Problemgruppen und somit einen sozialen Integrationsbeitrag leisten will, aber die Basis, nämlich Maßnahmenteilnehmer fehlen. Ein genereller Kritikpunkt der Beschäftigungsmaßnahmen lässt sich in der Diskussion finden, ob die Beschäftigten die Arbeit des qualifizierten Fachpersonals übernehmen. Dieses Argument wird von dem Gesprächspartner entkräftet, denn im eigenen Betrieb wurde eine Arbeitsübernahme durch das Projektklientel gewünscht:

„... der Druck, die dann doch einzusetzen, kommt aus dem Betrieb selber, also wir hatten hier schon mehrere Gespräche mit den Meistern und Vorarbeitern, die dann gesagt haben, wenn die eh da sind, dann können sie das doch machen." (I24)

Die Regelung, die daraufhin gefunden wurde, stellt einen zehnprozentigen Anteil an der Arbeit des Stammpersonals für die Maßnahmenteilnehmer dar. Dass dieser Anteil nicht überschritten wird, kontrolliert die Verbandsbehörde des Amtes. Kritik wird an der Maßnahmengestaltung von seiten des Amtes geübt, da durch die originär zugewiesenen 
Aufgaben, wie z. B. die Servicekräfte, die ausschließlich für die Kontrolle der Anlagen zuständig sind, keine Qualifizierung der Teilnehmer für den ersten Arbeitsmarkt stattfindet. Die Aussagen des Stammpersonals und des Amtsvertreters zeigen eine komplette Integration der Personen in die Arbeitsabläufe.

Abschließend bleibt festzuhalten, dass auch für die Fallstudie Düsseldorf ein großes Potential für soziale Integration nachgewiesen werden kann. Die aufgedeckte Situation in stimmt mit dem ersten Teil der formulierten Annahmen der Hypothese I überein. Die Verwaltung des Garten-, Grünflächen- und Forstamtes ist sich über den eigenen Beitrag zur sozialen Nachhaltigkeit und sozialen Integration durchaus bewusst, da ein Konzept zur Arbeitsförderung entwickelt wurde. Durch die Maßnahmen können die Teilnehmer Kompetenzen und Wissen erlernen und sich somit auf dem Arbeitsmarkt und im gesellschaftlichen Leben neu positionieren. Dies wird direkt und aktiv vom Garten-, Friedhofs- und Forstamt unterstützt, indem feste Stellen für dies Klientel geschaffen werden. So kann festgestellt werden, dass eine eingeschränkte Sichtweise bei dieser Institution nicht vorliegt, sondern der umfassende Ansatz zur Hilfe aktiv gefördert wird. Demnach stimmen die im zweiten Teil der Hypothese I formulierten Annahmen mit der hier aufgefundenen Situation nicht überein. Dennoch sollte erwähnt werden, dass die Konzeptionalisierung der Maßnahmen als Reaktion auf die politische Vorgabe der Stadt Düsseldorf, Beschäftigung zu schaffen, gesehen werden kann. Die nachhaltig angelegten und aufeinander aufbauenden Maßnahmen sind, dessen ungeachtet, als entscheidender Beitrag zur sozialen Integration von Langzeitarbeitslosen zu werten. 


\subsubsection{Fallstudie Berlin}

Tabelle 19: Institutionen in Berlin, deren Maßnahmen und Partner

\begin{tabular}{|c|c|c|}
\hline \multicolumn{3}{|c|}{ Berlin } \\
\hline Institution & Maßnahme / Projekt & Partner \\
\hline Landesforstamt & $\begin{array}{l}\text { - Waldschulen } \\
\text { - Umweltbildung für Problemgruppen (in } \\
\text { Planung) } \\
\text { - „Förster in die Schule“ (in Planung) } \\
\text { - „Arbeit statt Strafe“ } \\
\text { - „Außenkommando“ }\end{array}$ & $\begin{array}{l}\text { - verschiedene Schulen Berlins } \\
\text { - Quartiersmanagement (Projekt } \\
\text { „Soziale Stadt“) } \\
\text { - verschiedene Schulen Berlins } \\
\text { - Gerichte, Sozialämter, } \\
\text { Freigängerhaftanstalt }\end{array}$ \\
\hline $\begin{array}{l}\text { Grünflächenamt } \\
\text { „Berlin Mitte“ }\end{array}$ & $\begin{array}{l}\text { - Beschäftigungsprojekte für Straffällige } \\
\text { - Beschäftigungsprojekte für } \\
\text { Langzeitarbeitslose } \\
\text { (Arbeitsgelegenheiten mit Mehrauf- } \\
\text { wandsentschädigung; kurz: MAE) }\end{array}$ & $\begin{array}{l}\text { - Gerichte, Sozialämter } \\
\text { - Beschäftigungsgesellschaften, Job - } \\
\text { Center }\end{array}$ \\
\hline
\end{tabular}

Aus forschungsökonomischen Gründen hat in der Bundeshauptstadt Berlin ausschließlich die Befragung der "grünen Behörden“ stattgefunden. Die Projekte, die in oben stehender Tabelle aufgeführt sind, weisen einen Schwerpunkt in der Beschäftigungsförderung auf.

Das Landesforstamt Berlin betreibt eine breit gefächerte Umweltbildung in eigenen Waldschulen. Hier werden vornehmlich Schulklassen an das Ökosystem Wald herangeführt. Diese Schulen sind, nach eigenen Aussagen, in der Peripherie von Berlin angesiedelt (I25). Angedacht ist eine verstärkte Zuwendung der Umweltbildung auf soziale Problemgruppen. Hierzu soll das Projekt „Soziale Stadt“ als Verteiler dienen. In dem Bund-Länder-Programm „Soziale Stadt" geht es um Stadtteilsanierung auf ganzheitlicher Ebene, von der Gebäudesanierung bis hin zur Stabilisierung des sozialen Gefüges im Stadtteil. Die Bundestransferstelle „Soziale Stadt" beschreibt die Ziele des Programms wie folgt:

- „ „die physischen Wohn- und Lebensbedingungen sowie die wirtschaftliche Basis in den Stadtteilen zu stabilisieren und zu verbessern,

- die Lebenschancen durch Vermittlung von Fähigkeiten, Fertigkeiten und Wissen zu erhöhen,

- Gebietsimage, Stadtteilöffentlichkeit und die Identifikation mit den Quartieren zu stärken.“ (Bundestransferstelle Soziale Stadt, 2007)

Gerade in problematischen Stadtteilen, mit vielfältigen Problemen, sollen die Umweltbildungsmaßnahmen des Landesforstamtes ausgedehnt werden. Mit Hilfe des Quartiermanagers der Stadtteile soll der Kontakt zu Schulen und schulunabhängigen Gruppen aufgebaut und Angebote im bzw. rund um den Wald gefunden werden (I25). Da dieses Vorhaben noch mit einigen organisatorischen Problemen zu kämpfen hat, konnten 
hier noch keine Erfahrungswerte oder Effekte berichtet werden. Der Ansatz und die Zielsetzung, dem Quartiersmanagement „unter die Arme zu greifen“ (I25) und soziale Brennpunkte bewußt mit Angeboten anzusteuern, deutet auf eine hohe soziale Kompetenz und Verantwortung des Landesforstamtes hin.

Ein weiterer Punkt des Engagements des Landesforstamtes liegt in der Planung einer Aktion „Förster in die Schule“. Hier sollen Forstfachleute Unterricht bzw. Projekte in Schulen übernehmen. Dies soll gerade die Vermittlung fachspezifischer Themen betreffen, wie, z.B. Walbau, Einschlag und Jagd (125). Der Gesprächspartner ist hierbei der Meinung, dass diese Themen effektiver von Fachpersonal vermittelt werden können:

„,.., da wollen schon lieber wir kommunizieren, d.h. also mit unseren Förstern.“ (I25)

Ein weiteres Projekt des Landesforstamtes stellt die Maßnahme „Arbeit statt Strafe“ dar. Hier werden vornehmlich jugendliche Straftäter zu sozialer Arbeit verurteilt anstelle der Inhaftierung. Diese Arbeitsstunden konnten von den Verurteilten auf den Flächen des Forstamtes abgeleistet werden (I25). Aus der Arbeit mit dieser Klientel hat sich ein weiteres Projekt entwickelt. Das sogenannte „Außenkommando“ wird in Kooperation mit einer Freigängerhaftanstalt durchgeführt. Die Zielsetzung dieses Projektes ist die erste Stufe der Resozialisierung von Straffälligen (125). Die Personen kommen aus dem Vollzug in diese Freigängeranstalt, wo sie auf das Leben außerhalb der Haftanstalt vorbereitet werden. $\mathrm{Zu}$ dieser Vorbereitung gehört auch der Nachweis von geleisteter Arbeit außerhalb der Haftanstalt. Diese Arbeiten können auf den Flächen des Landesforstamtes organisiert und geleistet werden.

„Ja, jetzt sind wir sozusagen oben Außenkommando, sprich also ein wesentlicher Punkt zur Umsetzung dieser Freigänger $\ldots$ ja überhaupt des Freiganges als Strafvollzugsmaßnahme." (I25)

Diese Aussage macht die Bedeutung des Landesforstamtes in diesem Projekt deutlich. Denn ohne die Kooperation mit dem Forstbereich könnte die Art der Resozialisierung nicht stattfinden, da es, nach Aussagen des Forstexperten, kaum Anbieter von Arbeit für Straffällige im öffentlichen Raum gab (125). Über den immensen Einfluss auf soziale Eingliederungsprozesse ist sich die Forstpartie in diesem Falle bewusst, was aber in anderen Städten noch stärker genutzt werden könnte, da hier ein Teil dieses Potentials brach liegt.

Das Grünflächenamt im Bezirk Berlin Mitte weist ebenfalls Projekte auf, die Beiträge zur sozialen Integration leisten. Auch hier liegt der Schwerpunkt auf Beschäftigungsprojekten. Zum einen können ebenfalls auf den Flächen des Grünflächenamtes Straffällige, vor allem Jugendliche, ihre Arbeitsstunden bzw. Sozialstunden ableisten, zu denen sie verurteilt wurden. Diese Klientel wird in Arbeiten, wie Papierkörbe säubern, Laubfegen oder Rasenmähen eingebunden, wobei sie unter der Aufsicht des amtseigenen Personals stehen (I26). Als „Beschäftigungsstelle“ bezeichnet der Gesprächspartner das eigene 
Grünflächenamt für die Beschäftigungsmaßnahmen für Langzeitarbeitslose. Im Rahmen der Arbeitsbeschaffungsmaßnahmen (ABM) und Arbeitsgelegenheiten mit Mehraufwandsentschädigung (MAE) sind etwa 150 Personen im Grünflächenamt beschäftigt (I26). Bei letztgenannten Arbeitsgelegenheiten wird den erwerbsfähigen Hilfebedürftigen eine angemessene Entschädigung für Arbeiten gezahlt, welche die Kriterien des öffentlichen Interesses und der Zusätzlichkeit erfüllen. Zu beachten ist hier, dass es sich um eine zusätzliche Zahlung zum Arbeitslosengeld II handelt (SGB II, §16 Abs. 3). Diese Maßnahmen werden größtenteils von Beschäftigungsfirmen organisiert, begleitet und abgewickelt, so dass das Grünflächenamt nur noch die Fläche bzw. die Arbeit zur Verfügung stellt.

„Wir stellen die Arbeit zur Verfügung, wo sie arbeiten können, alles Andere, Arbeitsgeräte, Material, die ganze Abrechnung, Beaufsichtigung, Anleitung, das läuft dann über diese Firma, das sind uns eigentlich die Liebsten, wo wir praktisch nur den Nährwert haben, also die machen die Arbeit bei uns, aber die ganze Organisation und was da so an Man-power oder Frauen-power noch dahinter hängt, das bleibt eben bei der Firma." (I26)

Diese Aussage macht deutlich, dass es sich bei der Zusammenarbeit mit den Beschäftigungsfirmen für das Grünflächenamt zunächst um die Erledigung von Arbeiten handelt. Über den Effekt, den die Maßnahme für die Teilnehmer haben kann, wird an dieser Stelle keine Aussage getroffen. Aus den Maßnahmen können sich allerdings weitere Beschäftigungsverhältnisse auf dem Arbeitsmarkt ergeben. Bei den Teilnehmern dieser Maßnahmen handelt es sich in den meisten Fällen um Langzeitarbeitslose, die über diese Beschäftigungen für den Arbeitsmarkt qualifiziert und arbeitsfähig gehalten werden.

Die Beschäftigungsmaßnahmen umfassen im Grünflächenamt einen Zeitraum von sechs Monaten mit der Option zur Verlängerung um weitere drei Monate, wobei diese zu den kurzfristigen Maßnahmen gezählt werden können. In dieser Zeit kann, nach Meinung des Amtsvertreters, eine Integration in den ersten Arbeitsmarkt kaum stattfinden, aber die Struktur der Maßnahmen zeigt, dass eine Heranführung an das Arbeitsleben für die Langzeitarbeitslosen möglich ist.

„[...] Langzeitarbeitslosen, dass die überhaupt mal wieder lernen früh aufzustehen, fünf Tage in der Woche am Stück zu arbeiten [...]." (I26)

Auch diese Erlernung eines strukturierten Tagesablaufes kann als Beitrag zur Integration dieser Personengruppe gewertet werden. $\mathrm{Zu}$ den Personengruppen äußert sich der Gesprächspartner wie folgt:

„[...] im Prinzip haben die null Chancen und genauso verhalten die sich auch, das wissen die auch, wenn sie die hier sehen, mit welchem Elan die hier arbeiten, denen kann man, ich sage mal, beim Laufen die Schuhe besohlen." (I26)

Hier wird deutlich, dass den Personen auf dem Arbeitsmarkt keine Chance eingeräumt wird, auch nicht nach Absolvierung einer Beschäftigungsmaßnahme. Ob diese Aussage als Ablehnung der Personen oder als eine realistische Einschätzung der Situation zu werten ist, 
kann zu diesem Zeitpunkt nicht beantwortet werden. Da Äußerungen ein Indiz der sozialen Integration darstellen, findet sich im Zusammenhang mit dem Aufenthalt im öffentlichen Grünraum eine tolerierende Aussage.

„Ja, wenn die eine Nacht auf der Bank liegen, da wird keiner was sagen.“ (I26)

Hier wird der Bedarf an Schlafstätten für Obdachlose erkannt und die Nutzung des öffentlichen Raumes toleriert. Wird hierzu die allgemeine Haltung des Amtsvertreters den Beschäftigungsmaßnahmen gegenüber herangezogen, kann festgestellt werden, dass es sich um eine eher ablehnende Haltung handelt. Durch die Maßnahmen wird in der Realität kein Effekt auf dem ersten Arbeitsmarkt erzielt, was sich, nach Meinung des Gesprächspartners, z.T. aus dem Verhalten der Maßnahmenteilnehmer ergibt.

„Ja, ich bin da relativ leidenschaftslos, die Leute verhalten sich ja auch nicht unbedingt so als würden sie den Job behalten wollen." (I26)

Dennoch solidarisiert sich der Amtsvertreter auch mit der Personengruppe, da er auf die Bezahlung hinweist und betont, dass eine angemessene Entlohnung äquivalent zu den Lebenshaltungskosten gewährleistet sein muss (I26).

Die allgemeine Position eines Amtes kann ebenfalls einen Effekt auf die soziale Integration von Randgruppen haben. Das Landesforstamt Berlin versucht sich durch aktive Mitarbeit in städtische Belange einzubringen, wozu die Bemühungen für Randgruppen zu zählen sind. Werden daraufhin Langzeitarbeitslose in Beschäftigungsmaßnahmen eingesetzt, wird stets darauf geachtet, dass die Tätigkeiten nicht mit den Regeltätigkeiten des Stammpersonals in Konflikt geraten. Aus den zusätzlichen Arbeiten soll aber ein Nutzen für das Amt gezogen werden, indem Arbeiten, die sonst nicht verrichtet werden können von den Maßnahmenteilnehmern erledigt werden. Diese Projekte werden außenwirksam dargestellt „Tue Gutes und rede darüber." (126) und für die amtseigene Profilierung genutzt. Wobei hier eine klassische win-win-Situation angesprochen wird, nämlich eine Selbstdarstellung und Reputation für zukünftige Projekte auf Seiten des Landesforstamtes und die Beschäftigung von Langzeitarbeitslosen auf der Seite gesellschaftlicher Belange.

Das Grünflächenamt sieht die Beschäftigungsmaßnahmen eher kritisch, da kein Effekt erzielt wird und dies auf das soziale System mit seinen grundsätzlichen Fehlern zurückzuführen ist (I26). Nach Meinung des Amtsvertreters kann eine Integration der Arbeitslosen in den Arbeitsmarkt nicht stattfinden, da diese kurzfristigen Maßnahmen keine Entlastung für die Staatsausgaben darstellen, sondern eine erhöhte Belastung. Das System der 1-EUROJobber verleite Unternehmen dazu, die Arbeit nicht mehr mit dem eigenen Personal zu erledigen, sondern einen Großteil zu entlassen, die dann als 1-EURO-Jobber in den Betrieb zurückkehren und ihre vorherige Arbeit zu einem Bruchteil des vorherigen Gehaltes erledigen (I26). Da auch das Grünflächenamt an Sparmaßnahmen gebunden ist, besteht hier ebenfalls keine andere Möglichkeit, als Arbeiten von Beschäftigungsmaßnahmen 
übernehmen zu lassen. Insgesamt steht das Grünflächenamt den Integrationsmaßnahmen ablehnend gegenüber.

Der erste Teil der Hypothese I deckt sich mit der Situation der Fallstudie Berlin, denn das soziale Potential des Waldes gilt durch die Projekte als belegt. Es werden Unterschiede in der Wahrnehmung der sozialen Nachhaltigkeit und Integration zwischen den Institutionen festgestellt. Das Landesforstamt setzt die Maßnahmen bewusst zur Eigendarstellung ein und verkennt dadurch den Effekt, den die Beschäftigungsmaßnahmen für die Gruppe der Freigänger haben. Durch diese Stufe der Resozialisierung können die Teilnehmer Wissen und Kompetenzen erlernen, die innen die Wiedereingliederung in das gesellschaftliche Leben erleichtern. Möglicherweise wird so eine positive Bindung an den Wald erreicht und eine Identifikation mit dem gesellschaftlichen Gut und darüber hinaus mit der Gesellschaft allgemein ermöglicht. Der Schritt der Wiedereingliederung in die Gesellschaft würde sein Optimum in der Übernahme eines ehemals Straffälligen in ein Arbeitsverhältnis im Landesforstamt finden. Hierdurch wäre das Durchdringen des Begriffs der sozialen Nachhaltigkeit erwiesen. Da dies weder im Forst- noch im Grünflächenamt der Fall ist, muss auch hier von einem eingeschränkten Verständnis der Sozialfunktionen des Waldes und der Grünflächen gesprochen werden. So decken sich ebenfalls die Annahmen der Hypothese I im zweiten Tel mit der vorgefundenen Situation in Berlin.

\subsubsection{Fallstudie Nürnberg}

Tabelle 20: Institutionen in Nürnberg, deren Maßnahmen und Partner

\begin{tabular}{|l|l|l|}
\hline \multicolumn{2}{|c|}{ Nürnberg } \\
\hline \multicolumn{1}{|c|}{ Institution } & \multicolumn{1}{|c|}{ Maßnahme / Projekt } & \multicolumn{1}{c|}{ Partner } \\
\hline $\begin{array}{l}\text { mudra - Alternative } \\
\text { Jugend- und Drogenhilfe } \\
\text { e.V. }\end{array}$ & - Waldprojekte mit Suchtkranken & - Forstamt Nürnberg, Sozialamt \\
\hline
\end{tabular}

In Nürnberg konnte nur ein Projekt mit Waldbezug untersucht werden. Das Gespräch fand vor Ort im Wald statt, so dass keine Digitalaufnahme und somit keine Transkription des Gesprächs vorliegt. Die Auswertungen beziehen sich auf ein Gedächtnisprotokoll und Internetrecherchen. Das mudra Waldprojekt wurde vor 20 Jahren gegründet als Einrichtung zur Wiedereingliederung von Menschen mit einer Abhängigkeitserkrankung in das Arbeitsleben (mudra Jahresbericht 2006, S. 31). Es werden im Rahmen der beruflichen Rehabilitation verschiedene Forstarbeiten durchgeführt (mudra Waldprojekt, 2005), die folgende Arbeitsfelder umfassen: 
- „Forstliche Dienstleistungen: Aufforstungsarbeiten, Beseitigung von Wind und Schneebruch, Wegesicherung

- Brennholzproduktion, Verkauf und Auslieferung

- Vertrieb von Holz- und Rindenbriketts

- Natur- und Dekorationsmaterial

- Produktion von Grobmöbeln für den Außenbereich

- Spielgeräte, Holzspielzeug, Möbel, Reparaturarbeiten“ (mudra Jahresbericht 2006, S. 62)

Diese Arbeitsfelder werden mit einzelnen Arbeitsgruppen abgedeckt. Die Arbeiten werden auf den Flächen des Nürnberger Stadtwaldes durchgeführt in enger Kooperation mit dem zuständigen Forstbetrieb. Die Zielgruppe für diese Tätigkeiten sind ehemalige Abhängigkeitskranke und Menschen, die sich in Substitutionsbehandlungen befinden. Gerade für diese Menschen ist der Weg zur beruflichen Rehabilitation besonders schwer, denn welcher Arbeitgeber stellt ohne Schwierigkeiten Menschen mit einem Abhängigkeitshintergrund ein. Die Brennholzproduktion läuft seit mehren Jahren sehr erfolgreich, denn hier wird das Holz von den Projektteilnehmern selber eingeschlagen, zu einem zentralen Lagerplatz gefahren, dort gelängt, gespalten und zum Trocknen gestapelt (I27). Von dort aus wird das Brennholz direkt vermarktet, d.h. verkauft und ausgeliefert. Der Gesprächspartner weist in diesem Zuge auf die hohe Flexibilität der Vermarktung hin, denn es wird versucht, den Wünschen der Kunden individuell zu entsprechen. Gegen einen geringen Aufpreis wird das Holz sogar in den Lagerraum der Kunden gebracht und dort aufgestapelt (127). Die Flächen, auf denen das Brennholz anfällt, werden von dem ansässigen Förster ausgezeichnet und dann eigenständig von den Mitarbeitern des mudra Waldprojekts bearbeitet. In dieser Weise findet ebenfalls die Holzentnahme für die Schreinerei der Institution statt, in der die Grobholzmöbel für den Außenbereich gefertigt werden. Auch hier werden die Arbeiten in Verantwortung der mudra durchgeführt und mit dem eigenen Maschinenpark transportiert und verarbeitet. Die Stellensituation im Waldprojekt stellt sich wie folgt dar:

„3 Dauerarbeitsplätze (2 Wald, 1 Schreinerei)

9 ABM-Stellen für ALG II-EmpfängerInnen

4 ABM-Stellen für ALG I-EmpfängerInnen

ca. 4 Zusatzjobs nach SGB II § 16 (Ein-Euro-Jobs)“ (mudra Jahrebericht 2006, S. 62)

Diese Stellen unterliegen einer zeitlichen Begrenzung, die als kurz- bis langfristig bezeichnet werden kann. Der Erfolg der Rehabilitation hängt eben von diesem zeitlichen Rahmen ab. So kann die Aussage getroffen werden, je länger die Abhängigkeitskranken an einer Maßnahme teilnehmen können, desto besser stehen die Chancen für eine Weitervermittlung. In diesem Zuge wird von einem integrativen Erfolg nicht nur gesprochen, wenn die Teilnehmer in den Arbeitsmarkt vermittelt werden, sondern auch dann, wenn die Erhaltung der Arbeitsfähigkeit erreicht wird. Zudem sollen Schlüsselqualifikationen vermittelt werden, welche dem Klientel ermöglicht, überhaupt berufliche Bildungsmaßnahmen zu ergreifen (mudra Jahresbericht 2006, S. 32). Zu dem integrativen Erfolg der Arbeitsprojekte zählt auch die Einrichtung bzw. 
das Angebot von Lehrstellen für Drogenabhängige innerhalb der Institution. Erwähnenswerte Nebeneffekte der Beschäftigungsmaßnahmen sind eine sinkende Kriminalitätsrate und Rückfallquote der Drogenabhängigen. Der Begriff der Gesundheitsökonomie macht deutlich, dass Arbeitsprojekte dieser Art eine volkswirtschaftlich sinnvolle Alternative zur Arbeitslosigkeit darstellen (mudra Jahresbericht 2006, S. 32). Denn werden arbeitslose Menschen, auch jene mit Suchtkrankheiten, arbeitsfähig gehalten, fallen im Gesundheitssystem geringere Kosten an. Diese Entwicklung würde sich ebenso auf Senkung der Kosten für die Arbeitslosigkeit auswirken, die sich im Jahre 2004 auf 86 Mrd. EURO beliefen (Institut für Arbeitsmarkt- und Berufsforschung, 2007).

Im Rahmen des Gespräches mit dem Vertreter der Institution wurde der integrative Erfolg der Beschäftigungsmaßnahmen in der Schaffung einer festen Arbeitstelle im Waldprojekt gesehen, die von einem ehemaligen Maßnahmenteilnehmer bekleidet wird. Dieser aktive Beitrag zur sozialen Integration, nämlich die Vermittlung in den Arbeitsmarkt stellt nur einen kleinen Teil des Erfolges dar, da Menschen mit einem Suchthintergrund erschwert Arbeit finden. In erster Linie soll in den Arbeitsprojekten den Personen bei der Suchttherapie Hilfestellung geleistet werden und sie wieder an einen geregelten Tagesablauf gewöhnen. Unter diesen Voraussetzungen, arbeitsfähig und -willig zu sein, kann eine soziale Rehabilitation stattfinden.

„Arbeit ist nicht das Wundermittel gegen die negativen Folgen der Drogenabhängigkeit, aber eine wichtige Station auf dem Weg zur Rehabilitation." (mudra Jahresbericht 2006, S. 33)

Dieses Zitat macht die Haltung und Position der Institution deutlich, denn durch die Projekte im Wald können die vielseitigen Probleme von Suchtkranken nicht in ihrer Komplexität gelöst werden. Dennoch kann ein entscheidender Beitrag zur sozialen Integration, durch Tolerierung und Solidarität von Menschen mit Suchterkrankungen leisten. Die formulierte Annahme der Hypothese I muss für den ersten Teil als bestätigt angesehen werden, denn das große soziale Potential des Waldes liegt vor. Für den zweiten Teil der Hypothese gelten die Annahmen, bezogen auf die Situation des Projektes in Nürnberg, als nicht bestätigt. Der Grund hierfür liegt in der Tatsache, dass ausschließlich eine soziale Organisation Auskunft über das Projekt liefern konnte. Hier kann demnach nicht von einer eingeschränkten Sichtweise der sozialen Nachhaltigkeit und Integration gesprochen werden, da eben diese Ziele in den Projekten verfolgt werden. Eine Definition von sozialer Integration fällt einer solchen Institution wesentlich leichter als einer Forstverwaltung. Somit wird das Potential des Waldes genutzt, um sozial integrative Projekte umzusetzen und zur Neupositionierung der Personengruppen in der Gesellschaft und zur Identifikation mit dieser beizutragen. Bedauerlicherweise war es nicht möglich, die Forstseite zu diesem interessanten und erfolgreichen Projekt zu befragen. Da dies Projekt schon seit langen Jahren besteht, kann angenommen werden, dass die Forstverwaltungen zur sozialen Integration durch Toleranz 
und Solidarität mit der Problemgruppe der Suchtkranken beiträgt und diese unterstützt. Dennoch lässt die Bereitstellung der Flächen für das mudra Waldprojekte auf geringen Beitrag der Forstverwaltung schließen.

\subsection{Schlussbetrachtung der Hypothese I}

Die Annahme, dass im Wald ein großes Potential für soziale Nachhaltigkeit und soziale Integration vorhanden ist, kann durch die Analyse der o.g. Fallstudien als bestätigt angesehen werden. Das geringe Verständnis der kommunalen Forstverwaltung von sozialer Nachhaltigkeit und die daraus resultierende eingeschränkte Nutzung können in fünf von acht Fallstudien nachgewiesen und als verifiziert betrachtet werden.

Die Analyse der Interviews hat ergeben, dass die Forstverwaltungen dennoch einen Beitrag zur sozialen Integration von sozialen Rand- bzw. Problemgruppen leisten. Fraglich ist hierbei die vollständige Erschließung des integrativen Potentials des Waldes, da ein Defizit in der Definition von sozialer Nachhaltigkeit festgestellt wurde (s. Kap. 1.2). Die Grundvoraussetzung der sozialen Integration, eben die Solidarität mit Anderen und die Tolerierung der Anderen, gerade der Problemgruppen, konnte in der Analyse für die Forstverwaltungen nachgewiesen werden. Die Weiterführung der Integration, die sich in einer Anstellung oder weiteren Unterstützung der Personen auch über die Projekte hinaus, niederschlägt, kann nur in Ausnahmefällen (Freiburg, Düsseldorf, Nürnberg) aufgezeigt werden. Die Vermittlung von Wissen und Kompetenzen durch die Waldarbeit, die in anderen Berufen eingesetzt und genutzt werden können (Plazierung), wie z.B. Teamarbeit, können als Integrationsbeitrag (Kulturation) angesehen werden. Auch die Identifikation der Menschen mit dem Wald und die Interaktion zwischen den Menschen beeinflussen die soziale Integration positiv. Dies Verständnis von Integration konnte nur in den Ausnahmefällen bewiesen werden. In den übrigen Fallstudien ist die Bedeutung von sozialer Integration von den Forstverwaltungen nicht vollständig erschlossen worden.

Die Analyse der Maßnahmen hat ergeben, dass eine erfolgreiche Integration nur in Zusammenarbeit der Forstverwaltungen mit anderen Institutionen erreicht wird. Dies soll in den folgenden Kapiteln näher beleuchtet werden. 


\title{
5.5 Überprüfung der Hypothese II
}

\begin{abstract}
Hypothese II: a) Die Forstverwaltung kann trotz ihres eingeschränkten Verständnisses durch Kooperation mit anderen Institutionen Lösungen für die Nutzung des Waldes zur sozialen Integration finden.

b) Die Forstverwaltung kann durch die Kooperation mit anderen Institutionen neue Ressourcen erschließen und den eigenen Handlungsspielraum erweitern.
\end{abstract}

\subsubsection{Kooperation}

Um die Hypothese II zu überprüfen, lässt sich zunächst allgemein festhalten, dass die Projekte, die Integration zum Ziel haben und an denen der Forstsektor beteiligt ist, in allen genannten Fallstudien in Kooperation mit Ämtern vorwiegend des sozialen Bereiches ablaufen. Die Kompetenz, mit unterschiedlichen Problemgruppen umzugehen und auf die individuellen Bedürfnisse einzugehen, fehlt im Forstbereich. Da im Wald Potential für integrative Maßnahmen steckt, sollten starke Verbindungen geschaffen werden, um dieses Potential nutzbar zu machen. In den folgenden Kapiteln sollen Ausführungen über die Entstehung von Kooperation, die Kooperationsstruktur und die forstliche Beteiligung zur Hypothesenüberprüfung herangezogen werden. Nachfolgend werden die Faktoren nicht mehr für jede Fallstudie in einzelnen Kapiteln abgehandelt, sondern die Faktoren bilden die Kapitel und werden nach den bedeutensten Beispielen aus den Fallstudien ausgewertet.

\subsubsection{Beteiligte Ämter}

Die beschriebenen Fallstudien geben einen Überblick über die Integrationsprojekte und deren beteiligten Institutionen (s. Tabellen 13 bis 20). Als weitere Organisationen, mit denen eine Zusammenarbeit besteht, wurden in Freiburg von Seiten des Forstamtes die einzelnen Bürgervereine der Stadtteile genannt. Diese Angabe stand in keinem direkten Zusammenhang mit einem Projekt zur Integration. Bei den Bürgervereinen handelt es sich um freiwillige Zusammenschlüsse, die Interessen des Stadtteils vertreten (I15). Falls das Forstamt Aktionen und Projekte plant, wird der Bürgerverein befragt und schon zu Beginn einbezogen und eine enge Zusammenarbeit angestrebt. In Hamburg wurde ebenfalls ergänzend eine weitere Organisation genannt, die nicht spezifisch Integrationsprojekten zugeordnet werden können. So richtet die "Schutzgemeinschaft Deutscher Wald“ auf 
Flächen des Forstamtes die Waldspiele für Kinder und Jugendliche aus (I23). Diese Zusammenarbeit bedeutet für das Forstamt einen geringen Aufwand, denn hier werden lediglich die Flächen einer Revierförsterei zur Verfügung gestellt. Zusätzlich wurden in den Gesprächen keine Institutionen genannt, die an Integrationsprojekten in Kooperationen beteiligt waren.

\subsubsection{Kontaktaufnahme}

Für die Entstehung von Kooperation zwischen Institutionen ist zunächst interessant zu erfahren, von wem die Kontaktaufnahme zu Beginn einer Zusammenarbeit ausgeht. Bei dieser Frage traten unterschiedliche Möglichkeiten des Kontaktes auf, die über die Analysekategorien hinausgehen. Zum einen entstehen die Kontakte zur Zusammenarbeit aus vorhandenen Kontakten und Strukturen, die sich über eine lange Zeit aufgebaut haben. Von diesen Strukturen profitieren die Verantwortlichen der Institutionen und müssen die Kontakte nicht selbst aufbauen, sondern übernehmen. Dies lässt sich in der Theorie nach Benz (1994) wieder finden, denn es wird beschrieben, dass Institutionen leichter zu einer Kooperation bereit sind, wenn diese im Vorfeld bereits erfolgreich stattgefunden hat. Zum anderen sind private Kontakte und das Engagement einzelner Personen genannt, wodurch eine Zusammenarbeit entsteht. Die Variable der Kontaktaufnahme ist in vier Codes eingeteilt. Der erste Code bezeichnet die Kontaktaufnahme auf Anregung der Rand- bzw. Problemgruppen. Hierzu konnten in den Fallstudien keine Beispiele gefunden werden. Dies Ergebnis zeigt, dass die betroffenen Personengruppen keine Initiative ergreifen, Projekte im Wald zur Integration anzustoßen. Hieraus lässt sich die Frage ableiten: Benötigen diese Personen überhaupt Integrationsprojekte und Projekte mit Waldbezug? Es wird davon ausgegangen, dass dies ein häufiger Befund ist, denn den Betroffenen ist die eigene Situation nicht bewusst. So könnte z.B. einem Menschen, der als langzeitarbeitslos gilt, die eigene Randständigkeit zur bzw. Abgeschlagenheit von der Gesellschaft nicht bewusst sein. Aus diesem Empfinden heraus wird sich, nach Meinung der Verfasserin, keine Eigeninitiative entwickeln. Der zweite Code ist die Kontaktaufnahme auf Anregung einzelner Personen. Diese Form der Kontaktaufnahme tritt in den Fallstudien Göttingen, Freiburg und Berlin auf. In Göttingen ist dies von der Gesprächspartnerin von Arillus $\mathrm{GmbH}$ benannt, die mit der Idee, ein "Survival-Wochenende“ für Suchtkranke im Wald, an die Institutionen herangetreten ist und eine Kooperation hergestellt hat. Einzelne Personen zeichnen sich auch in Freiburg für das „Treffpunkte im Wald“ - Projekt verantwortlich. Hier werden von den Gesprächspartnern explizit der Jugendsachbearbeiter der Polizei und der ansässige Revierförster benannt. Diese Personen sind die treibenden Kräfte für das Projekt, ohne deren Anregung und 
Engagement die Zusammenarbeit nicht stattgefunden hätte. Auch in Berlin werden von Seiten des Landesforstamtes ausdrücklich engagierte Mitarbeiter genannt, die ein Projekt und damit die Kooperation von verschiedenen Institutionen möglich gemacht haben. Der dritte erwähnenswerte Code der Kontaktaufnahme geht von Seiten eines anderen Amtes aus. In dieser Kategorie sind die meisten Zuordnungen zu finden. Erwähnung sollen hier das Treffpunktprojekt in Freiburg finden, welches durch die Anregung der Polizei angestoßen wird, da für sie das Problem besteht, dass sich die Jugendlichen an unpassenden Orten aufhalten, aber keine Ausweichmöglichkeit vorhanden ist. So ist die Polizei an die Jugendförderung herangetreten, was eine sofortige Beteiligung des Forstbereichs nach sich zog. Die Beschäftigungsfirma AGIL mbH berichtet von einem lockeren Kontakt zum Forstamt zu Beginn der Kooperation. Nach einmaliger Projektdurchführung tritt nun das Forstamt an die Institution heran, um weitere Einsätze der Langzeitarbeitslosen zu planen. Auch die mudra in Nürnberg berichtet, dass das Forstamt zur Einsatzabsprache an die Institution herantritt. Das Beispiel aus Hamburg zeigt den umgekehrten Fall. Hier wird ausdrücklich berichtet, dass das Forstamt bzw. die Mitarbeiter im Revierdienst nicht aktiv auf die Problemgruppen und die Institutionen, die sich mit diesem Klientel beschäftigt, zugehen, sondern zurzeit nur auf Anfragen reagieren (I23). Die Kontaktaufnahme von Forstamtsseite in den Fallstudien zeigt eine erkennbare Struktur. Wenn das Forstamt den ersten Kontakt aufnimmt, geschieht dies fast ausschließlich in Projekten an denen Beschäftigungsfirmen beteiligt sind. Hieraus lässt sich ein gewisser Nutzen der Projekte für das Forstamt ableiten, denn es werden zusätzliche Arbeiten getätigt, die allein vom Forstamt bzw. mit den eigenen Mitarbeitern nicht zu leisten sind. Diese Aussage deckt sich mit den Zuordnungen des Codes Kontaktaufnahme von Seiten des eigenen Amtes, dem vierte Code der Variable. Hier spricht ein Vertreter einer Beschäftigungsfirma von Akquirierung der Arbeit, indem seine Organisation auf Behörden zugeht und nach nützlichen Beschäftigungsfeldern sucht (I22). Es liegt nahe, dass eine Firma, die Langzeitarbeitslose qualifizieren und beschäftigen will, sich zunächst um Einsatzfelder kümmern muss. Sind dieser Kontakt und Strukturen geschaffen, dann besteht auch Nachfrage auf der Forstamtsseite. Zusammenfassend bleibt festzuhalten, dass die kommunalen Forstämter in den seltensten Fälle Initiator der Projekte sind, sondern eher auf Anfrage reagieren. Kommt die erste Anregung von der Forstseite, dann handelt es sich meist um gewachsene Kontakte, die weiter genutzt werden oder engagierte Mitarbeiter, die eine neue Idee ins Leben rufen. Die Initiative wird häufig von den Organisationen ergriffen, die sich mit Problemgruppen auseinandersetzen. Ist die Forstpartie der Motor für Projekte, die Integration zum Ziel haben, geht dies mit einem wandelnden Selbstverständnis bzw. Aufgabenverständnis einher, dass sich z.B. in Freiburg gezeigt hat. 


\subsubsection{Ablauf von Kooperation}

Nach der Theorie von Benz (1994) ist Kooperation zwischen Partnern durch definierte Merkmale gekennzeichnet. Diese werden in strukturelle, prozessuale und ergebnisbezogene Merkmale unterteilt und aus diesem Grund wird von der Mehrdimensionalität von Kooperation gesprochen (Benz, 1994). Das Hauptaugenmerk liegt im Folgenden auf den Kooperationen an denen die Forstverwaltung beteiligt ist. Strukturelle Merkmale von Kooperation sind gekennzeichnet durch wechselseitige Gleichberechtigung der Kooperationspartner, die gleiche Verfahrens- und Entscheidungsrechte besitzen. Die Partner verfügen über gleiche Rechte und Pflichten während der Zusammenarbeit. Demgegenüber stehen Strukturmerkmale der Kooperation, wie einseitige Handlungsmöglichkeiten und Entscheidungsmacht oder gesetzliche Regelungen, die zeigen, dass ein Partner nicht „freiwillig“ oder sich aus freien Stücken für eine Kooperation entschieden hat (Benz, 1994). Die prozessualen Merkmale beschreiben den Umgang bzw. die Art der Kommunikation der Kooperationspartner. Hierbei ist zu klären, ob während der Zusammenarbeit eine dialogische Kommunikation herrscht oder ob nur ein Partner kommuniziert und die Gegenseite reagiert. Weiterhin ist festzustellen, inwiefern die Kontakte ablaufen. Gibt es in der Zusammenarbeit direkte Kontakte, sog. „face-to-face“ Kontakte oder findet dieser via Telefon oder e-mail statt und der persönliche Kontakt steht nicht im Vordergrund. Der Prozess der Kooperation ist weiterhin gekennzeichnet durch die Abstimmung zwischen den Partnern. Diese Abstimmung erfolgt als direkte Kommunikation über Ziele, Interessen, Problemdefinitionen, Situationseinschätzungen, Kenntnisse und Werthaltungen, mit dem Ziel diese in Einklang zu bringen (Benz, 1994).

Als letzte Dimension von Kooperation ist die Ergebnisorientierung zu nennen. Alle Akteure und Organisationen sollen sich hierbei freiwillig auf eine von allen akzeptierte, konkrete Lösung der Probleme einigen. Nur wenn eine von allen Beteiligten anerkannte gemeinsame Entscheidung durch Interaktions- und Kommunikationsprozesse erzielt wird, kann von kooperativen (Verwaltungs-) Handeln gesprochen werden (Benz, 1994).

Die Mehrdimensionalität von Kooperation wird in den Interviews durch einen Fragenkomplex erörtert, der in die Codes Struktur, Prozess und Ergebnis unterteilt ist. Zur Struktur von Kooperation wurden in den Fallstudien folgende Angaben gemacht. Die wechselseitige Gleichberechtigung der Kooperationspartner wurde von Seiten des Forstes in Hamburg im Beschäftigungsprojekt mit (ehemals) Suchtkranken als Beziehung „auf Augenhöhe, gegenseitig" (I23) bezeichnet. An anderer Stelle werden die gleichen Verfahrens- und Entscheidungsrechte mit dem Beispiel der Arbeitsteilung erläutert. In Düsseldorf kümmert sich die soziale Institution um die Anliegen der Klientel und entscheidet, welche Maßnahmen ergriffen werden müssen. Auf der anderen Seite entscheidet das Garten- Friedhofs- und Forstamt über die technische Ausstattung und den Einsatzort der Personen. Zwei 
Entscheidungsbereiche, die von unterschiedlichen Institutionen gedeckt werden. Natürlich steht hierbei die entsprechende Kompetenz der Beteiligten im Vordergrund und ist Basis für die Arbeitsteilung. Als häufigstes auftretendes strukturelles Merkmal von Kooperation lässt sich jedoch die einseitige Handlungsmöglichkeit ausmachen. Diese geht in der Regel mit der einseitigen Entscheidungsmacht einher. Vor allem in dem Projekt der Treffpunkte im Wald wird von den beteiligten Partner über einseitige Handlungsmöglichkeit berichtet. Die Jugendarbeiter sind aufgrund ihrer Ausbildung in der Lage mit den Jugendlichen zu kommunizieren (I14) und die Grundlage für dieses Projekt herzustellen. Die Einrichtung der Plätze im Wald lag in der Verantwortung und im Entscheidungsbereich des Forstamtes.

\footnotetext{
„Der Jugendsachbearbeiter der Polizei hat zwischendurch mal von einem Container im Wald geträumt, da hat natürlich der Förster gesagt, vollkommen unmöglich hier feste Installationen rein zu setzen, wir machen hier keine Fundamente, das wird alles so wassergebundene Oberflächenschichten gemacht, dass das alles reversibel ist, selbstverständlich." (I14)
}

Dieses Beispiel der Fallstudie zeigt auf, dass die beteiligten Institutionen, ihres Kompetenzbereiches entsprechend, Entscheidungen treffen, durchsetzen und diese auch von allen anderen Partnern akzeptiert werden.

Die prozessualen Merkmale einer Kooperation stellen die Kommunikationswege und die Abstimmung der Partner in den Vordergrund. In den Fallstudien ist ein Schwerpunkt in den „face-to-face“ Kontakten und der Abstimmung über Ziele, Interessen und Werte zu finden, was die aufgeführten Beispiele belegen sollen. In Göttingen sind in dem Suchttherapieprojekt mit Arillus $\mathrm{GmbH}$ und dem Forstbereich runde Tische zur Abstimmung der Interessen und Ziele veranstaltet worden (17). Gleiches gilt für die Kooperation zwischen dem Garten-, Friedhofs- und Forstamt in Düsseldorf und der Caritas im Bereich der Ausbildung im Gartenund Landschaftsbau. Da Praktikumsteile im Garten-, Friedhofs- und Forstamt stattfinden, muss über den Lehrplan Einstimmigkeit herrschen und setzt einen intensiven Kontakt voraus (I24). Als intensiv wird auch der Kontakt mit der Zukunftswerkstatt beschrieben. So wird der Einsatz der Servicekräfte laufend besprochen und die Effektivität für alle Beteiligten reflektiert. Dies kann nur in Abstimmung der Interessen und Ziele auf Seiten beider Institutionen erfolgen. Als Beispiel der Kommunikation in den Kooperationsprojekten sollen die "face-to-face" Kontakte in Hamburg zwischen Forstbereich und passage gGmbH gelten. Um die vereinbarte Aufgabe zu erfüllen, setzen sich hier die Vertreter der Institutionen zusammen und beraten über die Erfüllung der Aufgaben und erhalten eine Rückkopplung des Partners (123). Nur diese direkten Kontakte stellen die Grundlage einer kooperativen Aufgabenerfüllung dar (Benz, 1994). Die Kooperationsprojekte der weiteren Fallstudien weisen ähnliche prozessuale Merkmale auf, die z. T. als normale Arbeitskontakte beschrieben werden. Schwierig sind diese Merkmale festzustellen, wenn es sich um lange Zeit bestehende Projekte handelt, die eines intensiven Austausches nicht mehr bedürfen. Hier verlaufen die Kontakte sehr routiniert und häufig am Telefon oder per e-mail ab, wobei 
es sich meist nur um die Erneuerung von Daten, z.B. Namen oder Adressen der Projektteilnehmer handelt. Zusammenfassend lassen sich die theoriegeleiteten prozessualen Merkmale von Kooperation in den untersuchten Projekten aufdecken.

Die dritte Dimension von Kooperation bezieht sich auf das Ergebnis. Ziel ist hierbei die freiwillige Einigung der Partner auf eine gemeinsame Aufgabenerfüllung. Die Lösung eines Problems muss von allen Beteiligten akzeptiert sein. Nach der Analyse des Datenmaterials lässt sich dieses Merkmal ebenfalls in einem Großteil der Kooperationsprojekte aufzeigen. Hier sollen daher nur einige markante Beispiele dieser Kooperationsdimension beleuchtet werden. Das Kasseler Jugendamt spricht in der Vielzahl der Projekte und Partner von „ergebnisorientierten Lösungsversuchen“ für die Probleme, die häufig mit dem Instrument des Runden Tisches einher gehen. In Freiburg i. Br. wird im Zuge des Projektes „Treffpunkte im Wald“" von einer Problemlösung gesprochen, die von allen Partner akzeptiert ist. Dies kann nur erreicht werden, wenn die Beteiligten an diesem Prozess mitwirken. Laut Aussage der Vertretung der Jugendförderung finden zur Lösung des Problems, Aufenthalt von Jugendlichen an unangebrachten Orten, Treffen der beteiligten Organisationen statt. Hierbei wird die Lösung gemeinsam entwickelt, die durch den Beteiligungsprozess von allen akzeptiert wird. Die Mitwirkung aller Institutionen erweist sich für die Problemlösung als unerläßlich. In Zuge des Treffpunkteprojektes wird in Bezug auf das ergebnisorientierte Merkmal von Kooperation auch darauf hingewiesen, dass eine Lösung, die von allen betroffenen Gruppen akzeptiert wird, nicht immer erreicht werden kann, z.B. Anwohner, die sich über die Treffpunkte im Wald beschweren. Ist in einer solchen Situation kein Konsens möglich, gilt das Ziel bei dieser Interessensgruppe wenigstens eine gewisse Akzeptanz zu erwirken. Dies ist mit einem hohen Maß an Aufklärungsarbeit verbunden.

Ein Beispiel aus Kassel zeigt durch eine konkrete Problemlösung das Ergebnis von Kooperation. Ein Container für Jugendliche sollte aufgestellt werden, da Jugendliche sich am Rande des Parks aufhielten und ihnen keine Räumlichkeiten zur Verfügung standen. Einen Platz für diesen Container zu finden, stellte sich als schwierig heraus. Weder die Parkeigenschaft sollte beeinträchtigt werden, noch sollten sich die Anwohner belästigt fühlen. Räumlich wurde ein Platz für den Container am Rande des Parks gefunden und die Belästigung der Anwohner sollte durch eine zeitlich begrenzte Nutzung des Objektes verhindert werden (I12). Im Vordergrund dieses Projektes steht als ergebnisorientiertes Merkmal die konkrete Problemlösung. Zusammenfassend lassen sich für die Kooperationsprojekte aller Fallstudien die ergebnisorientierten Merkmale aufzeigen. Häufigstes Merkmal sind hierbei die konkreten Problemlösungen und die gemeinsamen Entscheidungen, die Ziel der Kooperationen sind. 


\subsubsection{Entstehung von Kooperation}

Da in den letzten Kapiteln festgestellt wurde, wie die Kontaktaufnahme zur Zusammenarbeit stattgefunden hat, bleibt die Entstehung von Kooperation zu klären. Warum entschließen sich kommunale Forstämter zur Kooperation mit anderen Institutionen um Integration zu gewährleisten bzw. einen Beitrag hierzu zu leisten? Nach Benz (1994) ist die Bereitschaft zur Kooperation das Ergebnis einer Strategieentscheidung. Strategien wiederum können als Mittel zur Verfolgung von bestimmten Zielen bezeichnet werden (Benz, 1994). Die Theorie gibt die Ausprägungen der Entstehung von Kooperation vor (s. Kap. 3.3.2). In den Fallstudien konnten hierzu Beispiele gefunden werden. In Frankfurt wird die Kooperation mit einem Elternverband von Kindern mit Behinderungen von Pragmatismus gelenkt. Die Anlage von behindertengerechten Pfaden hätte aus dem eigenen Haushalt des Forstamtes nicht finanziert werden können. Da die Nachfrage aber sehr groß ist, wird gemeinsam mit dem Elternverband ein Weg der Finanzierung gefunden. Das Handeln des Forstamtes kann hier als pragmatisch bezeichnet werden, da es auf die Realisierbarkeit des Projektes abgestellt ist. In dem Treffpunktprojekt für Jugendliche in Freiburg kann der Grund für die Entstehung von Kooperation seitens der Jugendförderung in der Informations- und Kenntnisbeschaffung gesehen werden. Der Revierförster, mit dem in diesem Fall kooperiert wird, ist zuständig für das angrenzende Revier, hat Kenntnisse über die Nutzung des Waldes durch die Anwohner und den Zustand der Waldlehrpfade (114). Zudem wird hier die Kommunikation zwischen Forstamt und unterer Naturschutzbehörde zur Informationsbeschaffung genutzt. Diese Informationen und Kenntnisse des Revierförsters sind für die Jugendförderung, als Projektkoordinator, unbedingt nötig, um das Aufenthaltsproblem der Jugendlichen zu lösen. So führt kein Weg an der Beteiligung des Forstes an diesem Projekt vorbei. In einem Projekt des Jugend- und Sozialamtes in Frankfurt im Rahmen der Ferienspiele für Kinder und Jugendliche kooperieren zwei Einrichtungen. Das Projekt soll Kinder mit und ohne Behinderungen zusammenführen und an einem Tag in den Ferien stattfinden. Da dem Jugend- und Sozialamt die Information und Kenntnis fehlt um entscheiden zu können, welche Art von Projekt mit behinderten Kindern durchgeführt werden kann, wird eine Kooperation mit dem CeBeeF eingegangen. Nach einem gemeinsamen Brainstorming (I18) hat sich ein Schwerpunkt herauskristallisiert und ein Tauchprojekt entsteht, das von beiden Institutionen betreut und vom CeBeeF hauptverantwortlich durchgeführt wird. Ohne die Kompetenzen des CeBeeF's hätte das Projekt nicht stattfinden können. Ein weiterer Faktor, warum sich Institutionen zu einer Kooperation entschließen, liegt in der Risikovermeidung. Hierbei sollen die Unsicherheiten über Ergebnisse und das Risiko des Scheiterns vermindert werden (Benz, 1994).

„[...] zweitens machen wir keine Fehler, wir haben nicht das Risiko, dass wir Energie und Geld aufwenden und hinterher war es ein Flop, [...]" (I15) 
Dieses Zitat zeigt eindeutig, warum sich das Forstamt in Freiburg in verschiedenen Projekten für die Kooperation mit anderen Institutionen, wie andere Ämter der Stadt oder soziale Organisationen, entschieden hat. Gleiche Gründe gelten für das Garten-, Friedhofs- und Forstamt in Düsseldorf. Das Amt geht die Kooperation mit Beschäftigungsträgern zur Integration von Langzeitarbeitslosen in den Arbeitsmarkt zur Risikovermeidung ein. Ursprünglich war eine Integration ohne die Hilfe und Mitarbeit von Beschäftigungsträgern geplant, aber die Lebensumstände der Personen sind so komplex, dass das GartenFriedhofs- und Forstamt der sozialen Betreuung nicht gerecht werden kann. Es kann eine Suchtproblematik oder die Schuldensituation der Klienten nicht auffangen und lösen. Um das eigentliche Ziel, nämlich die Integration der Personen in das Arbeitsleben nicht zu gefährden, muss sich das Amt mit einem Partner zusammenschließen, der eben diese Kompetenzen besitzt (124). Als weiterer Grund für die Entstehung von Kooperation ist das Treffen von Entscheidungen zu nennen. Nur wenn Institutionen miteinander arbeiten, können überhaupt Entscheidungen zur Lösung von Problemen getroffen werden. Dies ist der Fall im Treffpunktprojekt in Freiburg. Da ein Aufenthaltsort für Jugendliche gefunden werden muss, wird der Förster frühzeitig eingebunden, um zu entscheiden, ob eine solche Möglichkeit im Randbereich des Waldes besteht. Der Revierförster kann an dieser Stelle mit seinem fachlichen Hintergrund einen entscheidenden Beitrag zur Umsetzung des Projektziels beitragen, denn auf Basis seiner Fachkenntnis werden die Treffpunkte ausgewählt. In Frankfurt ist ebenfalls Kooperation zwischen dem Jugend- und Sozialamt und dem CeBeeF aufgrund der Entscheidungsfindung entstanden. Die Aktionen im Ferienprogramm für Kinder mit und ohne Behinderungen werden mit dem CeBeeF abgestimmt, denn nur die Mitarbeiter des CeBeeF können einschätzen was und wie viel den Kindern, die z.B. auf den Rollstuhl angewiesen sind, zugemutet werden kann. Somit hat der CeBeeF einen ausschlaggebenden Einfluss auf die Entscheidungen des Jugend- und Sozialamtes und erst im Austausch bzw. in Kooperation können diese Entscheidungen überhaupt getroffen werden. Das Engagement einzelner Personen kann ebenfalls als Faktor zur Entstehung von Kooperation beitragen. Aus dem persönlichen Kontakt zweier Vertreter unterschiedlicher Organisationen kann eine Zusammenarbeit in einem bestimmten Projekt entstehen. Dies ist der Fall in Göttingen zwischen dem Forstamt und der Arillus $\mathrm{GmbH}$. Aus beidseitigem Engagement entstehen so die Projekte zur Umweltbildung und das Therapieprojekt mit Suchtkranken (17).

Die aktive Einbringung von Institutionen kann ebenfalls der Auslöser von Kooperation sein. Hierbei handelt es sich um einen Faktor, der im Verlauf des Forschungsprozesses in das Kategoriensystem aufgenommen wurde. In Freiburg und Berlin nannten die jeweiligen Vertreter der Forstverwaltung die eigene aktive Einbringung in die Fragen der Stadt als Auslöser von Kooperationsbeziehungen. 


\begin{abstract}
„Also da schotten wir uns nicht ab und sagen, nein wir warten bis die Probleme an uns herangetragen werden, sondern wir versuchen selbst aktiv die Probleme zu erkennen und einen Beitrag zu leisten." (I15)
\end{abstract}

„Das ist von uns aktiv, ...."

Diese Zitate zeigen eine außerordentliche Betonung und Bedeutung der eigenen Initiative. In beiden Fälle haben sich die Forstämter aktiv in den Problemlösungsprozess eingebracht und werden diesen mitgestalten. Dies ist besonders hervorzuheben, da es zunächst nicht dem Selbstbild der traditionellen Forstverwaltung erspricht, die gesellschaftlichen und sozialen Probleme der Städte zu lösen bzw. hierzu einen Beitrag zu leisten. Nach der Theorie von Benz entscheiden sich Unternehmen bzw. Institutionen für Kooperation, um einen kontinuierlichen Einfluss auf politisch - administrative Entscheidungen zu erlangen (Benz, 1994). Dies kann durch die aktive Einbringung in die gesamtgesellschaftlichen Fragen und Probleme der Großstädte geschehen und gerade auch von Institutionen, die nicht direkt mit der Klientel in Verbindung gebracht wird. In den Interviews nennen die Gesprächspartner weitere Gründe, die zur Entstehung von Kooperation geführt haben bzw. führen können. In Bezug auf die Zusammenarbeit zwischen dem Forstamt Göttingen und Arillus GmbH wird als Ausgangsbasis Vertrauen genannt. Die durchgeführten integrativen Aspekte finden nur statt, weil dem jeweiligen Partner ausreichend Vertrauen entgegengebracht wird (17). Von öffentlichem Druck, der zur Entstehung von kooperativen Projekten führt, wird in den Projekten in Freiburg gesprochen. Zum einen haben massive Beschwerden der Bewohner im Stadtteil den Anstoss zu dem Treffpunktprojekt geliefert (114). Zum anderen hat als Reaktion auf den öffentlichen Druck ein Ortstermin mit anschließender Einigung auf die Aufrechterhaltung von Treffpunkten für Homosexuelle stattgefunden (I17). Diese Beispiele lassen erkennen, dass öffentliche Reaktionen Druck auf Institutionen ausüben und einen Grund für die Entstehung von Kooperationen darstellen. An das Garten-, Friedhofs- und Forstamt trat die örtliche Politik mit dem Beschluss der Eingliederung von Langzeitarbeitlosen durch Beschäftigungsprojekte heran (I24). An dieser Stelle kann von einer Erwartungshaltung der politischen Ebene gesprochen werden, welche die Institution veranlasst, in Kooperation mit sozialen Organisationen zu treten. Von freiwilliger Initiative zur Kooperation um Integration zu gewährleisten, kann hier nicht gesprochen werden. Zudem ist die Kompetenz der kooperierenden Institution ebenfalls als Grund für die Entstehung von Kooperation zu nennen. Gerade in Projekten mit sozialen Randgruppen verfügen einzelne Institutionen, gerade aus dem grünen Bereich, nicht über ausreichend Hintergrundwissen, um sich dieser Klientel zielführend und umfassend zu widmen. Im Falle der Beschäftigungsfirma Werkstatt Frankfurt e.V. entstehen Kooperationen mit anderen Ämtern der Stadt auf direkte Anfrage bei der Institution. Die Ämter verfügen über Projekte, die direkt vergeben werden können. Diese werden daraufhin häufig von der Werkstatt Frankfurt ausgeführt (I20). In welchem Maße es sich hierbei um die sogenannte Amtshilfe handelt, 
kann an dieser Stelle nicht geklärt werden. Zusammenfassend lassen sich unterschiedliche Faktoren zur Entstehung von Kooperation in den Fallstudien ausmachen. Ein Schwerpunkt liegt allerdings auf dem Faktor der Informations- und Kenntnisbeschaffung, der zur Kooperation zwischen Institutionen führt. Mit diesem Faktor geht der Kompetenzaustausch der einzelnen Institutionen einher, der für Projekte zur sozialen Integration gerade von Seiten des Forstbereiches unerlässlich ist.

\subsubsection{Interessen der Kooperationspartner}

Die Interessen der Kooperationspartner zur Lösung von Problemen können nach der Theorie verschiedene Ausprägungen aufweisen. Die Interessen der Akteure geben Auskunft darüber, wie sich die inhaltlichen Ziele der Akteure zueinander verhalten (Benz, 1994). Die vier Interessenskonstellationen sollen im Folgenden beschrieben werden: Identische Interessen können vollständig miteinander vereinbart werden, was bedeutet, was ein Akteur anstrebt, wollen die Übrigen auch. Auf das gleiche Ziel gerichtet bzw. in die gleiche Richtung wirken komplementäre Interessen. Hierbei unterscheidet sich aber der Bezugsgegenstand (ebd.). Treffen konkurrierende Interessen von Akteuren aufeinander, wird die Interessensbefriedigung des einen Akteurs durch die Handlungen des anderen Akteurs behindert (ebd.). Antagonistische Interessen von Akteuren schließen sich gegenseitig aus (ebd.). In den untersuchten Projekten der Fallstudien treten vor allem komplementäre Interessen der kooperierenden Partner auf. Die Interessen des Forstsektors und die der sozialen Einrichtungen sind auf das gemeinsame Ziel, die Integration von Problemgruppen, gerichtet. Hier besteht aber ein Unterschied im Bezugsgegenstand, der auf der einen Seite durch den Wald und auf der anderen Seite durch die soziale Festigung oder Therapie dargestellt ist.

\subsubsection{Verhandlungsmodus}

Haben Akteure ihre Kooperationsbereitschaft signalisiert, treten die potentiellen Partner in Verhandlungen. Bei den Verhandlungen über die Kooperationsziele zwischen Partnern werden drei Modi unterschieden. Die positionsbezogenen Verhandlungen haben zum Ziel die Partner zur Einwilligung in die jeweils eigenen Ziele zu bringen. Diese Einwilligungen werden durch Tausch erreicht, d.h. Akteure werden durch das Abrücken von Zielen von der Gegenseite entschädigt (Benz, 1994). Diese Form der Verhandlung konnte in den Fallstudien nicht aufgedeckt werden. Allerdings wurde der zweite Modus von 
Verhandlungen, die kompromissorientiert verlaufen, mehrfach genannt. Kennzeichen dieser Verhandlungen ist, dass alle beteiligten Akteure von ihren ursprünglichen Zielen abrücken und eine Übereinstimmung anstreben. Dies kann nur geschehen, wenn die eigenen Ziele und Forderungen nur teilweise umgesetzt werden (ebd.). Im Freiburger Treffpunktprojekt hat ein Kompromiss in der Auswahl und Ausstattung der Plätze stattgefunden, wobei der Förster einen entscheidenden Beitrag zu dem Kompromiss leistet.

„Also der Kompromiss in Bezug auf die Plätze und die Ausstattung der Plätze da war natürlich der Förster derjenige, der gesagt hat a) was geht von den gesetzlichen Grundlagen her, von dem was im Wald möglich ist und nicht möglich ist." (I14)

Auch das Forstamt in Freiburg konzentriert sich in der Lösung von Problemen auf unterschiedlichster Ebene auf die Kompromissfindung (115). Das Ergebnis von Verhandlungen zwischen Vertretern der Stadt Freiburg und der Schwulenvereinigung kann ebenfalls als Kompromiss bezeichnet werden. Das Problem der Nutzung einer öffentlichen Grünfläche durch viele gesellschaftliche Gruppen, speziell Junkies und Homosexuelle, wurde durch einen abgetrennten Bereich am Tage für die Drogenabhängigen und die Öffnung in der Nacht als Treffpunkte für Homosexuelle gelöst (I17). Hier rücken beide Verhandlungsparteien von ihrem eigentlichen Ziel, nämlich der ständigen Öffnung des Parkteils für alle Gruppen, ab und lassen sich auf diese Lösung des Interessensproblems ein. Der dritte Verhandlungsmodus in Kooperationsprozessen ist der integrative Konsens. Dies soll in verständigungsorientierten Verhandlungen erreicht werden, wobei Akteure Ergebnissen zustimmen sollen, indem sie sachlich überzeugt werden sollen (Benz, 1994). Dieser Lernprozess, an dem alle Akteure beteiligt sind, findet in offenen Diskussionen statt. Obwohl diese Form der Verhandlung nicht explizit in den Interviews genannt wird, kann davon ausgegangen werden, das ein Lernprozess bei den unterschiedlichen Institutionen stattfindet und sich die Konsensfindung in den Projekten zum Großteil durch offene Diskussionen ergeben. Dies geht mit der Kompetenz der einzelnen Institutionen einher. Die kommunalen Forstämter müssen sich auf einen Lernprozess einlassen, um die Ziele und Interessen der sozialen Einrichtungen nachvollziehen zu können. Im umgekehrten Fall müssen die sozialen Institutionen auf die Kompetenz der Forstverwaltung vertrauen und von ihr lernen. Nur wenn diese Voraussetzung gegeben ist und die Abstimmung der Ziele offen diskutiert wird, kann ein Projekt zur sozialen Integration von Problemgruppen in Kooperation im Wald stattfinden. 


\title{
5.5.1.7 Innovation durch Kooperation
}

Für die meisten untersuchten Institutionen stellt die Kooperation in den integrativen Projekten mit Wald- bzw. Grünbezug eine Neuerung im Arbeitsalltag dar. Im Forstbereich könnte sogar von einer neuen zusätzlichen Aufgabe gesprochen werden. Zur Verdeutlichung, wie die Innovationen gerade in diesem Sektor beschrieben werden, sollen drei Zitate angeführt werden.

\begin{abstract}
„Natürlich wäre auch wiederum der Förster nicht allein auf die Problematik aufmerksam geworden, denn dass im Stadtteil Jugendliche sitzen, geht inn ja auch erstmal nichts an. Und diesen aufmerksamen Blick auf den Stadtteil insgesamt zu haben, zu sehen, dass der Wald auch eine soziale Funktion für den Stadtteil hat, dass das also Zusammenhänge und Verbindungen gibt, das ist natürlich auch eine besondere Qualität gewesen, die also auch von Seiten des Försters mit eingebracht worden ist." (I14)
\end{abstract}

Diese Äußerung zeigt, dass ein solches Engagement des Forstbereiches noch nicht in Erscheinung getreten ist. Die Mitwirkung des Revierförsters bei der Problemlösung kann im Treffpunktprojekt in Freiburg als Innovation gesehen werden.

„Für uns in Hamburg war es innovativ, aber ansonsten denke ich, ist es ein Projekt, was bundesweit schon oft gelaufen ist. Für uns war das ein einmaliger Versuch damals." (I23)

Der Einsatz einer Beschäftigungsfirma, die mit (ehemals) Suchtkranken arbeitet, zur Entfernung der Traubenkirsche mit dem Ziel der sozialen Integration stellt für das städtische Forstamt eine Neuerung des Arbeitsablaufes dar. Sich darauf einzulassen und den Problemgruppen eine Möglichkeit zur Beschäftigung zu geben, stand vor dem Projekt nicht im Blickpunkt des Forstes.

„[...] innovativ war es in dem Moment, wo wir so eine aufeinander aufbauende Maßnahme hatten, das war neu." (I24)

Die Maßnahmen des Garten-, Friedhofs- und Forstamts gelten als innovativ, da die Beschäftigungsmaßnahmen unter eigener Mitwirkung neu strukturiert worden sind. Kennzeichen der Projekte ist nun die unterschiedliche Betreuungsintensität von z.B. den Servicekräften und den Personen in der Ausbildung. Außerdem zählt die „Durchgängigkeit“ (I24) der Projekte, d.h. eine Person kann bestimmte Maßnahmen durchlaufen und dann eine Festanstellung in dem Amt bekommen, als immenses innovatives Kriterium. So gelten zusammenfassend die meisten Projekte, auch diejenige, die hier keine Erwähnung finden, als Innovationen im Aufgabenverständnis der Institutionen. Sowohl die kommunalen Forstverwaltungen lassen sich auf innovative Projekte im Wald ein, als auch die sozialen Institutionen, die in ihrer Aufgabenerfüllung einen innovativen Waldbezug herstellen. 


\subsubsection{Merkmale einer erfolgreichen, integrativen Maßnahme}

Die Frage nach den Merkmalen einer erfolgreichen, integrativen Maßnahme wird von den befragten Institutionen sehr unterschiedliche beantwortet. Zunächst muss eine Klärung des Erfolgsbegriffes erfolgen, um die Antworten der Experten richtig interpretieren zu können. Unter Erfolg wird die Erreichung der Ziele verstanden. Bei der Erreichung von Zielen spielen Erfolgsfaktoren eine wesentliche Rolle, denn sie begünstigen die Zielerreichung. Zudem treten im Prozess der Zielereichung positive Nebeneffekte auf, die kein angestrebtes Ziel darstellen, jedoch positiv zu bewerten sind. Im Folgenden sind die Antworten der Experten, nach den angestellten Vorüberlegungen, den Kategorien Erfolg, Erfolgsfaktoren und positive Nebeneffekte zugeteilt. In der nachstehenden Tabelle ist der Faktor Erfolg und die Häufigkeit der Nennungen der einzelnen Kategorien des Faktors aufgelistet.

Tabelle 21: Erfolg der Maßnahmen

\begin{tabular}{|l|c|}
\hline \multicolumn{1}{|c|}{ Erfolg } & $\begin{array}{c}\text { Anzahl der } \\
\text { Nennungen }\end{array}$ \\
\hline Integration in den ersten Arbeitsmarkt & 5 \\
\hline Qualifikation & 2 \\
\hline Durchhalten im Projekt & 1 \\
\hline Soziale Stabilität & 1 \\
\hline Beschäftigung & 1 \\
\hline Ersatzarbeit & 1 \\
\hline
\end{tabular}

Der Erfolg der Maßnahmen liegt in der Erreichung der Ziele. Das wesentliche Ziel der Kooperationsprojekte ist die Integration von Problemgruppen in die Gesellschaft. Dieses Ziel wird auf unterschiedliche Arten erreicht. Einen Schwerpunkt zur Zielerreichung stellen die Beschäftigungsprojekte dar. Der größte Erfolg der Beschäftigungsprojekte wird in der Integration der Maßnahmenteilnehmer in den ersten Arbeitsmarkt gesehen, was an der Häufigkeit der Nennung zu erkennen ist. Eine weitere Ausprägung des Erfolgs von Beschäftigungsmaßnahmen stellt die Qualifikation der Teilnehmer dar. Nur wenn Personen nach einem Projekt eine Bescheinigung über die Weiterentwicklung ihrer Fähigkeiten vorlegen können, haben sie eine Chance auf dem (ersten) Arbeitsmarkt eine Anstellung zu finden. Das Durchhalten der Teilnehmer über die gesamte Projektdauer hinweg ist als Erfolg der Maßnahme zu werten. Können die Personen z.B. ein Beschäftigungsprojekt zu Ende bringen ohne längere Ausfallzeiten nachzuweisen, gilt dies als Schritt zur gesellschaftlichen Integration. Ein weiterer Erfolg von Integrationsprojekten ist die soziale Stabilität. Hierbei besteht der Erfolg darin, dass nach einer Maßnahme die Schulden eines Teilnehmers überschaubar und die Tilgung geregelt sind, dass gerade bei suchtkranken Personen auf die Gesundheit geachtet wird und wieder einigermaßen geregelte Lebensumstände herrschen, 
wie die Pünktlichkeit am Morgen zum Arbeitsantritt. Sind diese Grundlagen durch eine Maßnahme geschaffen, kann die Integration der Personen in die Gesellschaft erfolgen. Weiterhin äußert eine Beschäftigungsfirma, dass der Erfolg für einige Personen allein schon darin besteht, überhaupt eine Beschäftigung zu haben, auch wenn dies nur für einen befristeten Zeitraum gilt. Wenn die Integration in den ersten Arbeitsmarkt über Beschäftigungsprojekte für einige Teilnehmer unrealistisch ist, so ist der Einsatz für Ersatzarbeiten, wie z.B. das Freischneiden von Magerrasenflächen, als integrativer Erfolg zu bewerten. Für einige Langzeitarbeitslose, die entweder einen Sucht- oder Straffälligkeitshintergrund besitzen, muss diese Form der Arbeit als Integrationserfolg gelten, denn sie werden nur in den seltensten Fällen in Arbeitsverhältnisse auf dem ersten Arbeitsmarkt aufgenommen.

Die Erfolgsfaktoren, die das Ziel Integration begünstigen, sind in der nachfolgenden Tabelle aufgeführt.

Tabelle 22: Erfolgsfaktoren der Maßnahmen

\begin{tabular}{|l|c|}
\hline \multicolumn{1}{|c|}{ Erfolgsfaktoren } & $\begin{array}{c}\text { Anzahl der } \\
\text { Nennungen }\end{array}$ \\
\hline Motivation & 5 \\
\hline Perspektiven bieten & 4 \\
\hline Finanzieller Anreiz & 3 \\
\hline Akzeptanz & 3 \\
\hline Persönlicher Kontakt & 2 \\
\hline Beteiligung der Betroffenen & 2 \\
\hline Sinnvolle Arbeiten & 2 \\
\hline Angemessene Arbeiten & 1 \\
\hline
\end{tabular}

\begin{tabular}{|l|c|}
\hline Sichtbarer Erfolg & 1 \\
\hline Gemeinsames Ergebnis & 1 \\
\hline Verständnis & 1 \\
\hline Konflikte vermeiden & 1 \\
\hline Systemorientierung & 1 \\
\hline Kontrolle & 1 \\
\hline Anleitung & 1 \\
\hline Rückkopplung & 1 \\
\hline
\end{tabular}

Die Erfolgsfaktoren beschreiben auch hier schwerpunktmäßig Merkmale von Beschäftigungsprojekten. Der Erfolgsfaktor, der die häufigste Erwähnung in den Interviews findet, ist die Motivation der Maßnahmenteilnehmer. Da sich dieser Faktor auf Beschäftigungsmaßnahmen bezieht, besteht die Motivation vor allem in der Abwechslung der Arbeiten, d.h. nicht über Monate die Durchführung von identischen Arbeiten. Die Motivation der Personen geht einher mit dem Erfolgsfaktor Perspektiven, welcher den zweiten Rang in der Nennungshäufigkeit belegt. Werden nach den Beschäftigungsmaßnahmen Perspektiven geboten, wie z.B. eine weitere Arbeitsmöglichkeit, ist eine Voraussetzung für den erfolgreichen Abschluss einer Maßnahme gegeben, d.h. das Ziel der Integration könnte erreicht werden. Der Faktor Bezahlung bzw. finanzieller Anreiz trägt, nach Aussagen der Gesprächspartner, ebenfalls einen entscheidenden Teil zum Erfolg der Maßnahme bei. Wird der Einsatz der Arbeitskräfte honoriert, steigt die Motivation und die Maßnahme wird in den meisten Fällen erfolgreich abgeschlossen. Einen weiteren 
Erfolgsfaktor stellt die Akzeptanz für integrative Maßnahmen im direkten Umfeld der Teilnehmer und Projekte dar. Somit wird der Erfolg der Projekte gesteigert. Als Beispiel ist die Treffpunkteinrichtung für Jugendliche zu nennen, wobei in der gesamten angrenzenden Nachbarschaft für Akzeptanz geworben werden musste, um dieses Projekt erfolgreich durchzuführen. Der persönliche Kontakt zu den Projektteilnehmern kann als Erfolgsfaktor einer integrativen Maßnahme gesehen werden. Nur durch die direkten Kontakte und den persönlichen Austausch kann die Situation der Projektteilnehmer eingeschätzt und effektiv Hilfe angeboten werden. Als Erfolgsfaktor wird ebenfalls die Beteiligung aller Betroffenen beschrieben, denn nur so wird gewährleistet, dass sich die unterschiedlichen Gruppen mit den Projekten identifizieren und Projekte einen nachhaltigen Bestand haben. Sinnvolle und angemessene Arbeiten in den Beschäftigungsprojekten werden als Erfolgsfaktoren von Beschäftigungsprojekten bezeichnet. Die Arbeit sollte demnach sinnvoll sein, d.h. für die kooperierende Institution von Bedeutung sein. Z.B. ist hier die Entfernung der Traubenkirsche im Forstamt Hamburg zu nennen, die ohne den Einsatz der passage Mitarbeiter nicht geleistet werden könnte. Der Aspekt der angemessenen Arbeit zielt auf den Umfang und die Überschaubarkeit der Arbeiten. Mit den ungelernten Arbeitskräften sind Tätigkeiten auszuführen, die nicht zu anspruchsvoll sind und das Leistungsvermögen der oft körperlich sehr eingeschränkten Teilnehmer nicht übersteigen. Ebenso kann als Erfolgsfaktor von integrativen Maßnahmen die Sichtbarkeit des Einsatzes und die Gemeinschaft angesehen werden. Ist bei Beschäftigungsmaßnahmen das Ergebnis direkt sichtbar, wird die Motivation der Teilnehmer und somit der Integrationserfolg erhöht. Auch die Schaffung eines gemeinsamen Ergebnisses führt zur Motivation und ist als Erfolgsfaktor eines Projektes zu nennen. Von Seiten des Forstsektors wird der Erfolgsfaktor Verständnis genannt. Denn nur wenn die Positionen und Interessen des Gegenübers verstanden werden, seien es die der kooperierende Institution oder die der Problemgruppe, kann ein Projekt Erfolg haben. Ein Abwägen der Interessen der Beteiligten bei der Planung neuer Maßnahmen trägt zur Vermeidung von Konflikten bei, sichert so die Durchführung der Aktionen und sollte als Erfolgsfaktor nicht unberücksichtig bleiben. Ein Erfolgsfaktor für Integrationsprojekte ist die Systemorientierung, d.h. die Aktionen müssen durchführbar sein, auch wenn Maßnahmenteilnehmer, z.B. aufgrund von erfolgreichen Bewerbungen auf dem ersten Arbeitsmarkt, ausfallen. Eine Orientierung der Projekte auf einzelne Personen stellt einen Unsicherheitsfaktor dar und kann, nach deren Ausscheiden aus dem Projekt, zum Abbruch der Maßnahme führen (122). Gerade in Beschäftigungsprojekten stellen die Kontrolle und die Anleitung der teilnehmenden Personen Faktoren dar, die zu einem erfolgreichen Projektabschluss führen. Im Beschäftigungsprojekt „Entfernung der Traubenkirsche“ wurde die Gruppe der arbeitenden Personen zunächst von dem passage gGmbH Mitarbeiter und dem ansässigen Revierförster angeleitet und im Verlauf des Projektes auch kontrolliert. Fehlt 
diese Kontrolle, kann das erwünschte Arbeitsziel nicht erreicht werden (I23). Dies ist für die kooperierenden Institutionen unbefriedigend und kann keinen Beitrag zur Integration der Personen leisten. Ein wesentlicher Erfolgsfaktor ist die Rückkopplung der Kooperationspartner. Hierbei stehen das gemeinsame Ziel und das gegenseitige Interesse im Vordergrund. Wird den Partnern und Maßnahmenteilnehmer vermittelt, dass das Projekt für alle Beteiligten von Bedeutung ist und ein Ziel verfolgt wird, sind alle Seiten motiviert und tragen ihren Teil zum Gelingen der Maßnahme bei (123). Zusammenfassend kann eine Vielzahl von Erfolgsfaktoren aus dem Datenmaterial gewonnen werden, die zum Ziel der Integration von Problemgruppen beitragen. Bei der Analyse der Faktoren ist ein Schwerpunkt in den Beschäftigungsmaßnahmen zu erkennen. Demnach beeinflussen die Erfolgsfaktoren vor allem das übergeordnete Ziel der Integration in den Arbeitsmarkt positiv. Viele der Erfolgsfaktoren sind dennoch auf eine große Bandbreite von integrativen Maßnahmen anzuwenden. Denn die Motivation der Projektteilnehmer aufrecht zu erhalten, gilt nicht nur für Beschäftigungsmaßnahmen.

Neben den Erfolgsfaktoren und der Erreichung der Ziele treten häufig positive Nebeneffekte der Maßnahmen auf. Bei der Analyse der Integrationsmaßnahmen wurde ein Nebeneffekt aufgedeckt. Der positive Nebeneffekt von Integrationsmaßnahmen ist in der Entstehung von Einzelerfolgen zu sehen. So können Erfolge von Integrationsprojekten auch ganz individuelle Merkmale aufweisen, die als Nebenerfolge im Vergleich zu dem eigentlichen Integrationsziel zu bewerten sind. Dementsprechend ist eine Aktion erfolgreich, wenn eine Person, die als Schläger bekannt ist, im Projekt eine gute Teamarbeit zeigt oder wenn ein körperlich und geistig behinderter Mensch es schafft, über einen Baumstamm zu klettern (I7).

Abschließend bleibt festzuhalten, dass die Tabelle 22 der Erfolgsfaktoren hier als richtungweisend gelten soll, denn durch Beachtung einiger dieser Erfolgsfaktoren im Vorfeld der Projekte, können Integrationsprojekte zu einem erfolgreichen Abschluss gebracht werden. Sicher ist auch, dass die Liste noch um weitere Merkmale ergänzt werden kann und hier nur ein Ausschnitt der Möglichkeiten präsentiert wird. Dennoch werden, nach Meinung der Verfasserin, die wichtigsten Faktoren genannt, die den integrativen Erfolg von Projekten unterstützen. Hier sind vor allem die Gewinnung der Akzeptanz aller Beteiligten und Betroffenen, deren Einbindung in die Projekte und der persönliche Kontakt mit den einzelnen Gruppen zu nennen. Werden diese und weitere Faktoren beachtet, ist die Basis für einen integrativen Erfolg geschaffen. 


\subsubsection{Nutzen von Kooperation}

Die Analysekategorie Nutzen der Kooperation wird durch einzelne Codes beschrieben. Der Code, dem die meisten Aussagen der Interviewpartner zugeordnet werden konnten, ist die Steigerung der eigenen Position. Die Theorie unterscheidet hier die Positionssteigerung der Institution und die der ausführenden Person, die durch die Kooperation versucht, die eigene Stellung innerhalb der Institution zu stärken. In der vorliegenden Arbeit sind ausschließlich Aussagen zur Kategorie Positionssteigerung des Amtes / der Institution gefunden worden. Die Steigerung der Position des Amtes / der Institution liegt in unterschiedlichster Ausprägung vor. So erfährt das Forstamt Göttingen eine Positionssteigerung durch Bürgerbeteiligungen, die in Form von Umfragen stattfinden. Diese positiven Ergebnisse werden in der Öffentlichkeit dargestellt und unterstützen das Amt in seiner Positionierung. Durch diese Art der Rückkopplung mit den Nutzern des Waldes, kann auf deren Wünsche und Anregungen reagiert werden (15). Die Steigerung der Position des Amtes wird in Freiburg durch die Verleihung eines Preises betont. Für das Beschäftigungsprojekt für problematische Jugendliche erhalten das Forstamt und der Beschäftigungsträger den ersten Preis des Umweltministeriums. Diese Auszeichnung hat eine positive Wirkung in der Öffentlichkeit und trägt zum Image des Forstamtes bei. Eine positive Präsenz in und die Aufmerksamkeit der Öffentlichkeit sind wichtige Faktoren, um die Position der eigenen Institution zu steigern. Das folgende Zitat soll nochmals verdeutlichen, wie sich die Aufgaben und somit auch die Position des Forstamtes verändert.

„[...] Das, wir brauchen die als Problemlöser, als Einrichtung, die Konflikte steuert und moderiert und das ist die sicherste Möglichkeit zu überleben. Allein mit dem Forstbetrieb hätten wir uns nicht halten können." (I15)

Sich demnach aktiv an dem Problemlösungsprozess innerhalb der städtischen sozialen Probleme zu beteiligen, stellt in Freiburg eine entscheidende Veränderung im eigenen Aufgabenverständnis dar. In Berlin ist der Vertreter des Landesforstamtes überzeugt, dass die Position des Amtes mit der Anzahl der Fürsprecher zusammenhängt. Hat sich das Amt einen öffentlichkeitswirksamen Status erarbeitet, wird es den regierungsabhängigen Veränderungen in der Behördenstruktur standhalten können.

„Wenn man weiß, man hat so ein paar Fürsprecher, die das gar nicht so schlecht finden, wie das läuft, ist das schon mal gut, dann ist man vor diesen völlig spontanen Sachen (Umstrukturierungen, Budgetierung (Anmerk. d. Verf.)) eigentlich gefeit." (I25)

Die Kooperationen zwischen dem Forst- und dem sozialen Sektor in den integrativen Projekten führen nach der Analyse der Aussagen zu einer Steigerung der Position der Institution.

Die Erweiterung des Handlungsspielraumes wird z.B. in der Fallstudie Berlin als Nutzen der Kooperationsprojekte genannt. In Bezug auf die Zusammenarbeit mit der Freigängerhaftanstalt spricht der Vertreter des Landesforstamtes von einer Erweiterung des 
Wirkens im Wald, die nur mit Unterstützung der Personen der Haftanstalt stattfinden kann. Zum einen wird hier der Handlungsspielraum an direkten Tätigkeiten im Wald, wie Pflegemaßnahmen in Jungbeständen, vergrößert (125). Das Forstamt ist demnach in der Lage, diese Arbeiten überhaupt zu erledigen und kann ebenfalls den Handlungsspielraum auf die öffentliche Diskussion über soziale, gesellschaftliche Integration ausweiten, was nicht originär zum Aufgabenbereich des Forstsektors zählt.

Ein weiterer forstlicher Nutzen aus den Kooperationsprojekten ist der Zugang zu finanziellen Ressourcen. In Berlin werden Projekte genutzt, um Aufwendungen, die originär mit betrieblichen Mitteln gedeckt werden müssen, durch eben diese Projektmittel zu ersetzen (I25). Hierzu ist es notwendig, erfolgreich Fördermittel zu akquirieren.

Viele der interviewten Institutionen berichten von den Kontakten für weitere Projekte als Nutzen aus den Kooperationsprojekten. Auch hier soll die Aussage des Landesforstamtes Berlin beispielhaft für den forstlichen Nutzen stehen.

„[...] und wir gehören mit zu den Leuten, wo sie gute Erfahrungen gemacht haben, also sagen sie, Forsten muss das machen, dann machen wir das Ding auch irgendwie so mit. Ich finde, das ist schon eine gute Reputation [...]." (I25)

Tritt das Forstamt in einem Kooperationsprojekt als Partner auf und ist dies erfolgreich verlaufen, kann der Kontakt für weitere Projekte genutzt werden. Im nächsten Integrationsprojekt wird mit Wahrscheinlichkeit wieder auf den Forstsektor zurückgegriffen, da Strukturen und Mechanismen der Institutionen bekannt sind. Nutzt ein Forstamt ein Kooperationsprojekt bzw. die Kontakte des Projektes für weitere Maßnahmen, kann es ein fester Bestandteil der Kooperationsbeziehung werden. Dies wird von der Theorie von Benz (1994) bestätigt, die besagt, dass sich Institutionen eher für eine Kooperation entscheiden, wenn diese schon einmal erfolgreich stattgefunden hat. Diese Kontakte sollten die Forstämter für weitere integrative Projekte nutzen und nicht als lästige Pflicht sehen.

Der Synergieeffekt als forstlicher Nutzen von integrativen Kooperationsprojekten soll an dem Beispiel aus Freiburg aufgezeigt werden.

„Und natürlich für den Verein selbst, ist es eine Finanzierungsmöglichkeit, da ist es auch
eine Frage der Wirtschaftlichkeit und für uns ist es ein doppelter Synergieeffekt, wir
haben Jungbestandspflege und Durchforstungsflächen damit durchgearbeitet, also wir
sparen Pflegekosten bei uns und haben einen wirklichen pflegerischen Nutzen, einen
erheblichen sogar und der ist doppelt, dass sie im Winter Pflegeflächen durcharbeiten
und im Sommer haben wir natürlich überall die Problematik an den
Erholungsschwerpunkten, dass es jeden Tag aussieht, wie wenn eine Wildschweinhorde
durchgegangen sei und da schicken wir dann die Leute hin und die räumen das wieder
auf.“ (I15) Von einem Synergieeffekt wird hier als Vorteil aus der Kooperation für die beteiligten Institutionen gesprochen. Die Beschäftigungsfirma hat den Vorteil, dass sie ihre Klientel auf den Flächen des Forstamtes beschäftigen kann und somit eine Finanzierungsmöglichkeit gefunden hat. Das Forstamt profitiert durch jahreszeitliche Tätigkeiten von dem Kooperationsprojekt. Zum einen durch die Pflegemaßnahmen, wodurch erhebliche Kosten 
eingespart werden und zum anderen durch die Aufräumarbeiten an Erholungsschwerpunkten, die mit eigenem Personal nicht zu leisten sind. Diese Vorteile für die Kopperationspartner können als Synergieeffekte bezeichnet werden, denn ohne die Zusammenarbeit der Institutionen treten diese erst gar nicht auf.

Neben Synergieeffekt, Kontakten und Steigerung der eigenen Position gilt der Lerneffekt aus kooperativen Beziehungen ebenfalls als Nutzen für die einzelnen Partner.

„Es gibt viele Leute, die verstehen von einzelnen Fragen vielmehr als wir und da kann man auch was lernen und die können auch von uns was lernen. Und das kriegt man am besten hin, indem man so einen offenen Dialog hat, in dem man sich da austauscht." (I15)

Der Gesprächspartner aus dem Forstamt Freiburg macht deutlich, dass die unterschiedlichen Kompetenzen der kooperierenden Institutionen einen Lerneffekt nach sich ziehen. Von der fachlichen Qualifikation der Organisationen können die Kooperationspartner wechselseitig profitieren, wobei die Voraussetzung hierfür die Bereitschaft einen offenen Dialog zu führen, gegeben sein muss. Das Forstamt kann in den konkreten integrativen Projekten lernen, was es heißt mit einer Klientel von auffälligen, schwer erziehbaren Jugendlichen zu arbeiten und diese sinnvoll zu beschäftigen. Erlernen Forstämter generell Kompetenzen im sozialen Bereich, können sie aktiv zum Erfolg von integrativen Maßnahmen beitragen. Das Wissen der Verantwortlichen der kommunalen Forstämter über Maßnahmen, die für bestimmte Problemgruppen geeignet sind und welche Hilfestellungen sie selbst und andere Institutionen geben können, macht sie zu einem kompetenten Partner in integrativen Kooperationsprojekten. Der Lerneffekt sollte bewusst zur Erlangung von sozialen Kompetenzen genutzt werden.

Die Entscheidung zur Kooperation mit anderen Institutionen kann den Nutzen der Akzeptanz nach sich ziehen. Zum einen geht es um die Akzeptanz des jeweiligen Projektes (I15). Denn nimmt sich das Forstamt allein problematischen Gruppen an und will zur Problemlösung beitragen, wird dies Projekt weniger Akzeptanz in der Öffentlichkeit hervorrufen, als die Kooperation mit Institutionen des sozialen Sektors. Zum anderen wird die Akzeptanz der Einrichtung durch die Kooperation gestärkt.

„Das fördert die Akzeptanz einer Einrichtung, einer Verwaltung, das prägt, das wirkt in die Breite und das kommt ganz gut so." (I15)

Die bewusste Entscheidung für die Kooperation in integrativen Projekten bringt der Institution eine breite Akzeptanz in der Öffentlichkeit. Auch die Bevölkerung einer Stadt ist sich in gewisser Weise über die Aufgaben eines Forstamtes bewusst und die Hinwendung zu sozialen Problemen und deren Lösungen werden sicherlich besser angenommen, wenn die Zusammenarbeit mit Institutionen des sozialen Sektors stattfindet. So trägt Kooperation zur Annerkennung des Projektes und der eigenen Einrichtung bei.

Zu dem Nutzen von Kooperation aus forstlicher Sicht sind weitere Aussagen getätigt worden, die den vorhandenen Kategorien des Analyseschemas nicht zugeteilt werden konnten. Im 
Folgenden sollen diese kurz angesprochen werden. Als ein wichtiger Nutzen von Kooperationsprojekten wird die Konfliktvermeidung genannt. Hier werden durch die Zusammenarbeit Fehler vermieden, die entstehen könnten, wenn sich nur eine Institution einseitig mit der Lösung des Problems beschäftigen würde. Mit der Kombination von Kompetenzen, die Einrichtungen auf unterschiedlichen Fachgebieten in das Projekt einbringen, werden Fehlschläge direkt zu Beginn vermindert. Die Identifikation der Menschen mit dem Wald ist als weiterer Nutzen zu nennen. Durch die Beteiligung von Personengruppen an Projekten und Aktionen im Wald kann ein positives Gefühl entstehen, dass sich nachhaltig auf die Meinungsbildung und das Verhalten gegenüber dem Wald auswirken kann. Wird diese Identifikation erreicht, ist es von Nutzen für die Forstbehörde, die hierdurch Fürsprecher gewinnt.

„Selbst die Dinge, wenn man sie initiiert, aber dann versucht nur noch zu moderieren, es nur noch laufen zu lassen und es zu steuern, das zahlt sich sehr gut aus. Das ist eigentlich der größte Nutzen. (I15)

Dies Zitat von dem Vertreter des Forstamtes Freiburg nennt einen Nutzen von großer Bedeutung. Die Projekte, die von der Forstamtsseite z.T. ins Lebens gerufen, durchgeführt wurden und bei einer Wiederholung nur noch moderiert und gesteuert werden, können als „Selbstläufer" bezeichnet werden. Der Nutzen entspricht dem relativ niedrigen Aufwand von Forstamtsseite in der Wiederholungsphase und der weiterhin großen Öffentlichkeitswirksamkeit der Projekte. Der Nutzen, der die häufigste Nennung in den Fallstudien erfährt, ist der, dass zusätzliche, einfache Arbeit im Wald und dessen direkter Umgebung erledigt wird. Dabei sind z.B. das Entfernen der Traubenkirsche (Hamburg), das weißen einer Unterkunft (Frankfurt), Freischneidearbeiten (alle untersuchten Städte), Pflegezustände beibehalten (Berlin) zu nennen. Diese zusätzlichen Arbeiten können die Forstämter mit der derzeitigen Personaldecke nicht leisten. So spricht der Großteil der Vertreter der Institutionen von klassischen win-win-Situationen. Das Forstamt kann über integrative Beschäftigungsprojekte Arbeiten durchführen, die im Arbeitsalltag des Amtes nicht erledigt werden können und die Beschäftigungsträger haben die Möglichkeit ihre Klientel auf den Waldflächen sinnvoll zu beschäftigen. So profitieren alle Beteiligten von der Kooperation in den Projekten.

Zusammenfassend lässt sich festhalten, dass der Nutzen, den die kommunalen Forstämter aus den verschiedenen Kooperationsprojekten ziehen können, unterschiedliche Ausprägungen aufzeigt. Schwerpunkte des Nutzens liegen in der Steigerung der Position des eigenen Amtes durch die Beteiligung an integrativen Projekten und der Erledigung der Arbeiten im Wald, die ohne die Kooperation mit Beschäftigungsträgern nicht stattfinden könnten. 


\subsection{Schlussbetrachtung der Hypothese II}

Hypothese II: a) Die Forstverwaltung kann trotz ihres eingeschränkten Verständnisses durch Kooperation mit anderen Institutionen Lösungen für die Nutzung des Waldes zur sozialen Integration finden.

b) Die Forstverwaltung kann durch die Kooperation mit anderen Institutionen neue Ressourcen erschließen und den eigenen Handlungsspielraum erweitern.

Die Annahmen der Hypothese II können nach der Analyse des Datenmaterials in der Gesamtbetachtung als bestätigt angesehen werden. Die aufgedeckten Integrationsprojekte mit Beteiligung der Forstämter finden alle in Kooperation mit Institutionen statt, die aus dem sozialen Bereich stammen. Die Unkenntnis über Faktoren der sozialen Integration zwingt die Forstverwaltung dazu, Kooperation mit Institutionen einzugehen, deren Fachgebiet die Unterstützung von sozialen Problemgruppen ist. Zu bemerken ist an dieser Stelle, dass die Kooperation in Integrationsprojekten für einige Institutionen, gerade die Forstverwaltungen, eine Innovation im eigenen Aufgabenverständnis darstellt. Zuvor wurde nicht darüber nachgedacht, solche Projekte in die Aufgabenerfüllung aufzunehmen. Fraglich ist, ob die kommunale Forstverwaltung die Integration von Problemgruppen in die Gesellschaft als neue Aufgabe ansieht. Die Kontaktaufnahme für Projekte im Wald wird in den meisten Fällen nicht von der Forstverwaltung forciert, was auf einen geringen Aufwand seitens der Forstverwaltung für den Eintritt in Kooperationsbeziehungen hinweist. Ausnahmen bilden hier ausgewählte Projekte in Freiburg und Nürnberg, wo gerade die Forstverwaltung die Initiative für die Zusammenarbeit ergriffen hat. Warum sich die Institutionen zu Kooperationen entschließen, hat unterschiedliche Gründe. Der Schwerpunkt der Kooperationen dieser Untersuchung liegt auf der Informations- und Kenntnisverschaffung und in der Kompetenzverteilung der einzelnen Institutionen. Kompetenzen und Information werden als Ressourcen bezeichnet, die eine Institution zur Durchsetzung der eigenen Ziele einsetzen kann. So kann die Forstverwaltung mit der Information und der Kenntnis aus den Integrationsprojekten neue Ressourcen erschließen und den eigenen Handlungsspielraum erweitern. Die Erfolgsfaktoren der Integrationsprojekte stellen ein Beispiel hierzu dar. Die Kenntnis über diese Faktoren, versetzt die Forstverwaltung in die Lage, als kompetenter Partner in zukünftigen Projekten mit dem Ziel der Integration aufzutreten. Ein entscheidender Aspekt der Kooperationsbeziehungen zwischen den Forstverwaltungen und den Institutionen des sozialen Sektors ist im Nutzen der Kooperationsprojekte zu sehen. Die Forstverwaltungen können durch die Kooperationen den eigenen Handlungsspielraum erweitern, indem sie auf gesellschaftliche Themen innerhalb der Stadt Einfluss nehmen können und in Bereichen präsent sind, die zuvor nicht zum direkten Aufgabenbereich 
gehörten. Bei der Interpretation des Nutzens für die Institutionen darf sicher nicht außer Acht gelassen werden, dass sich Institutionen in erster Linie für eine Kooperation entscheiden, weil diese Vorteile für die eigene Verwaltung verspricht. Die Vorteile für die Forstverwaltungen liegen vornehmlich in der Steigerung der Position des eigenen Amtes innerhalb der städtischen Ämterstruktur und in der Verrichtung zusätzlicher Tätigkeiten, die aus eigener Kraft (personell und zeitlich) nicht zu bewältigen sind.

\title{
5.7 Überprüfung der Hypothese III
}

\begin{abstract}
Hypothese III: a) Die Forstverwaltung kann durch die Vermarktung des Produktes „Integration“ Lösungen zur Nutzung des sozialen Potentials des Waldes bieten.

b) Die Forstverwaltung kann durch die Vermarktung des Produktes "Integration“ neue Ressourcen erschließen und den eigenen Handlungsspielraum erweitern.
\end{abstract}

\subsubsection{Neues Handlungsfeld / Produkt}

Nachdem festgestellt werden konnte, dass Forstverwaltungen einen Beitrag zur sozialen Integration leisten können, stellt sich die Frage nach einem neuen Produkt, das sich aus der Teilnahme an Integrationsprojekten die kommunalen Forstämter entwickeln kann. Ist die Forstverwaltung durch Integrationsprojekte in der Lage, ein neues Aufgabenfeld zu definieren, Ressourcen zu erschließen und den eigene Handlungsspielraum zu erweitern? Zunächst soll die finanzielle und personelle Ausstattung der Maßnahmen der Fallstudien untersucht werden.

\subsubsection{Finanzielle Ausstattung der Maßnahmen}

Für die finanzielle Ausstattung der Projekte sind zunächst einige allgemeine Finanzierungsformen zu nennen. In Freiburg werden die Projekte, die im Umfeld des neuen Stadtteils Rieselfeld stattfinden mit einem Budget von mehr als 100.000 EURO finanziert (I15). In Frankfurt a.M. existiert ein Etat der Stadt in Höhe von 100.000EURO. Dieser steht für innovative, trägerübergreifende Projekte zur Verfügung. Institutionen können Anträge an die Grundsatzabteilung der Stadt stellen, die eine detaillierte Projektbeschreibung und die Kostenkalkulation beinhalten muss (I19). In entgegengesetzte Richtung hat sich die Finanzierung des Projektes in Hamburg entwickelt. Die Mittel für die Entfernung der 
Traubenkirsche wurden eingestellt, so dass das Projekt beendet werden musste (I23). Weiterhin sollen die einzelnen Finanzierungsformen der Maßnahmen analysiert und diskutiert werden.

Tabelle 23: Finanzierungsformen der Projekte und Anzahl der Nennungen

\begin{tabular}{|l|c|}
\hline Finanzierungsform & $\begin{array}{c}\text { Anzahl der } \\
\text { Nennungen }\end{array}$ \\
\hline ARGE (Job-Center) & 6 \\
\hline Mittel der Stadt & 6 \\
\hline Sponsoring & 4 \\
\hline EU - Mittel (z.B. ESP) & 3 \\
\hline Eigenfinanzierung & 3 \\
\hline Landkreise & 2 \\
\hline
\end{tabular}

\begin{tabular}{|l|c|}
\hline Soziale Stadt & 1 \\
\hline Beiträge & 1 \\
\hline $\begin{array}{l}\text { Ausgleichs- und } \\
\text { Ersatzmaßnahmen }\end{array}$ & 1 \\
\hline Wohnungsbaugesellschaften & 1 \\
\hline
\end{tabular}

In der Tabelle findet sich eine Vielzahl von Finanzierungsformen. Im Folgenden sollen für die Formen der Finanzierung einige markante Beispiele genannt werden.

Ein Schwerpunkt der Finanzierung von Beschäftigungsmaßnahmen in den Fallstudien liegt bei den Arbeitsagenturen bzw. den Job - Centern der Städte. Hier werden vornehmlich die Maßnahmen für Langzeitarbeitslose finanziert. Die Beschäftigungsfirmen erhalten eine Fallkostenpauschale von ca. 290 EURO für jeden Teilnehmer (I22), die den Aufwand der Arbeiten finanzierbar machen.

Ein weiterer Finanzierungsweg für integrative Projekte sind die direkten Mittel der einzelnen Städte bzw. der verschiedenen Ämter der Stadt. Z.T. werden hierbei die Lohnkosten der Maßnahmenteilnehmer in Beschäftigungsprojekten von den Sozialämtern mitfinanziert. Dem Jugendamt in Kassel steht ein Stadtetat von 7.000 EURO für Maßnahmen auf Grünflächen zur Verfügung. Das o.g. Budget der Stadt Frankfurt in Höhe von 100.000 EURO für trägerübergreifende integrative Maßnahmen stellt eine hervorragende Möglichkeit zur Finanzierung dar. Die Stadt Nürnberg stellt ebenfalls Mittel für Investitionen der Institution mudra, z.B. den Kauf von Schleppern für die Waldarbeit, bereit (I27).

Als wichtige Finanzquelle ist das Sponsoring zu nennen. Für Projekte werden Partner aus unterschiedlichen Bereichen angeworben, die mit finanzieller Unterstützung das Projekt ermöglichen. Für die Sponsoren ist dies ein Teil der Öffentlichkeitsarbeit und den Projektverantwortlichen ist es möglich, teure Ausrüstung zu kaufen, das z.B. für die Übernachtung im Freien mit Suchtkranken benötigt wird, wie Schlafsäcke, Geschirr etc. (17). Auch die integrativen Ferienspiele in Frankfurt werden durch Sponsoren unterstützt, die im aktuellen Programmheft Erwähnung finden (I18). Aus Sponsorenmitteln konnte ebenfalls in Frankfurt die Anlage eines behindertengerechten Spielplatzes finanziert werden (I21).

Eine Finanzierungsquelle für sozial integrative Projekte stellen die Förderprogramme der Europäischen Union dar. Der Europäische Sozialfonds (ESF) fördert z.B. das 
Beschäftigungsprojekt für Straffällige in Göttingen der Institution KiK e.V. in Bezug auf Personal- und Sachmittel. Nach Aussagen der Gesprächpartner wäre das Projekt ohne diese Unterstützung nicht möglich (12). Der ESF fördert ebenfalls die Aktivitäten des GartenFriedhofs und Forstamtes in Düsseldorf zur Wiedereingliederung von Langzeitarbeitslosen (124).

Die Fallstudien weisen die Bedeutung der Eigenfinanzierung der Maßnahmen durch die Institutionen auf. So finden sich die Institutionen z.B. in Kassel und Düsseldorf in der Situation wieder, die Lohn- oder Materialkosten der Maßnahmen aus den eigenen Haushaltsmitteln zu zahlen. Gerade die Lohnkosten der Beschäftigungsmaßnahmen werden in einigen Fällen auch von den jeweiligen Landkreisen getragen, die einen Auftrag zur Förderung der Beschäftigung auf regionaler Ebene haben (12, I24).

In Göttingen werden über das Bund-Länder-Programm „Soziale Stadt“ die Projekte in einem benachteiligten Stadtteil mit dem Ziel der qualitativen Stadtteilverbesserung finanziert. Hier werden insgesamt 16 Mio. EURO zur Sanierung des Stadtteils zur Verfügung gestellt. Zu bemerken ist hier, dass das Stadtforstamt diese Finanzquelle nicht für eigene Projekte oder eine Beteiligung nutzt. Als weitere Finanzierungsformen sind die Beiträge der Maßnahmenteilnehmer, was in der Umweltbildung eine wesentliche Rolle spielt (17), zu nennen. Ein Budget von Ausgleichs- und Ersatzmaßnahmen, aus dem Maßnahmen im Wald bezahlt werden (115) und eine Wohnungsbaugesellschaft, die einen Obolus an die Beschäftigungsfirma zahlt, die in einem Areal Müll aus den Grünanlagen sammelt (I22), stellen ebenfalls Finanzierungsformen dar.

In den oben aufgeführten Fällen handelt es sich zum größten Teil um die direkte Maßnahmenförderung in Bezug auf die Beschäftigungsprojekte (I14, I15, 124). Wobei die Gesprächspartner bei der Bezahlung der Projekte häufig darauf hingewiesen haben, dass es sich nicht um eine Vollfinanzierung, sondern um die Aufwandsentschädigung für die Anfahrt oder Werkzeuge handelt $(110,122)$. Die Frage nach der finanziellen Ausstattung der einzelnen Projekte identifizierte weitere Beispiele. So werden die Kosten der Maßnahme mit Suchtkranken im Freien je nach Aufwand der durchführenden Organisation berechnet und gestalten sich demnach variabel (17). Hat eine Institution mehr Aufwand in einem Kooperationsprojekt, so kommen ihr auch ein entsprechend größerer Teil der Mittel zu (I14). Ein wichtiger zu erwähnender Punkt ist die Bezahlung der Tätigkeiten von Beschäftigungsfirmen durch die Forstämter. In zwei Fallstudien fließen Geldmittel für die Arbeiten, die im Wald mit Problemgruppen verrichtet werden (I15, I23). In der Fallstudie Hamburg zahlt das Forstamt für das Entfernen der Traubenkirsche einen Betrag von ca. 180 EURO (360 DM, I23) pro ha an die Beschäftigungsfirma passage gGmbH. Wird abschließend die gesamte finanzielle Ausstattung der einzelnen Projekte betrachtet, muss festgestellt werden, dass die kommunalen Forstverwaltungen keine finanziellen Ressourcen 
durch die Kooperation in Integrationsprojekten erschließen können. Im Gegenteil wird aufgezeigt, dass die Forstämter die Leistungen der Institutionen erkaufen. Eine Aussage, die dem entgegensteht, ist der Vorstoß des Forstamtes in Göttingen, die eine Nutzungsgebühr der Umweltbildungseinrichtung erhebt, welche die Infrastruktur (z.B. Hütten) des Forstamtes nutzt (15). Das Landesforstamt in Berlin verweist auf die Akquirierung von Drittmitteln, um bestimmte Zielsetzungen auf den Waldflächen umsetzen zu können (I25). D.h. hier müssen Gelder angeworben werden, um z.B. zusätzliches Personal zu beschäftigen, die Tätigkeiten im Bereich Naturschutz leisten. Die aufgeführten Fallbeispiele zeigen deutlich, dass die Integration von Problemgruppen keine Ressource darstellt, die den Forstverwaltungen im kommunalen Raum finanzielle Mittel einbringt.

\subsubsection{Personelle Ausstattung der Maßnahmen}

Nach der Betrachtung der finanziellen Ausstattung der Projekte zur sozialen Integration schließt sich eine Analyse der personellen Ausstattung dieser an. Diese Variable soll Aufschluss darüber geben, wie hoch der Einsatz an Personal ist, um ein integratives Projekt durchzuführen. Die Angaben zeigen einerseits den Personalaufwand von Seiten der durchführenden Institutionen und andererseits die Personenzahl, die an den Maßnahmen teilnimmt. Außerdem variieren die Ausführungen hinsichtlich der Aussagekraft, von ganz konkreten Zahlen bis hin zu sehr unscharfen Angaben. Dies sei an zwei Beispielen verdeutlicht.

\footnotetext{
„Im Moment fünf ABM Kräfte, die das ganze Jahr über arbeiten, ein Gärtner als Fachanleiter, 1 Sozialarbeiter als Anleiter, der den Gärtner anleitet, wie er mit den Leuten umgehen muss." (I2)
}

„Das geht natürlich nur mit viel Personal [...]." (17)

Im ersten Beispiel handelt es sich um das Beschäftigungsprojekt für Straffällige in Göttingen, wo die genaue Anzahl der beteiligten Personen benannt werden kann. Im zweiten Beispiel wird der personelle Einsatz sehr vage beschrieben. Hierbei geht es um den Aufwand für eine Umweltbildungsveranstaltung mit behinderten Menschen. Insgesamt kann für diese Variable festgestellt werden, dass gerade die Beschäftigungsprojekte eine hohe personelle Ausstattung erfordern, da es sich meist um eine große Anzahl an Maßnahmenteilnehmern handelt, wie z.B. 30 Beschäftigte im Bereich Garten-Landschafts-Bau der Werkstatt Frankfurt e.V., die von vier Personen betreut werden. Der Betreuungsaufwand richtet sich zudem nach dem Hintergrund der Klientel, denn suchtkranke Projektteilnehmer benötigen umfassendere Hilfestellung als „normale“ Langzeitarbeitslose. Es bleibt hier zusammenfassend zu konstatieren, dass der personelle Aufwand für die integrativen Projekte einerseits von der 
Teilnehmerzahl abhängt und andererseits von der Klientel mit ihren individuellen Problemen und Schwierigkeiten. Weiterhin lassen sich Äußerungen der Gesprächspartner zum zeitlichen Aufwand der kooperativen Projekte finden. Gerade das Treffpunktprojekt in Freiburg ist mit einem hohen zeitlichen Aufwand verbunden. Die Vertreterin der Jugendförderung beschreibt den zeitlich intensiven Beginn des Projektes, der von Koordinations- und Konzeptionsgesprächen geprägt ist. In der Koordination und Leitung nimmt das Projekt pro Woche ein bis zwei Stunden Arbeitszeit in Anspruch (114). Der pädagogische Mitarbeiter des Projektes verwendet dagegen fünf Stunden pro Woche für dieses Projekt. Der Gesamteindruck, der in den Fallstudien entsteht, verstärkt den allgemeinen zeitlichen Mehraufwand zu Beginn aller kooperativen Projekte, denn hier findet die Abstimmung der einzelnen Institutionen über die Ziele der Maßnahmen, die Durchführung und die Arbeitsaufteilung statt. Findet eine Maßnahme zum wiederholten Male statt, kann diese Phase übergangen werden und der zeitliche Aufwand der Beteiligten sinkt.

\subsubsection{Erschließung eines neuen Handlungsfeldes}

Nachdem der Aufwand von Kooperationsprojekten auf verschiedenen Ebenen betrachtet wurde, schließt sich die Frage an, wie sich integrative Maßnahmen und Projekte in das Aufgabenfeld der kommunalen Forstverwaltungen einfügen. Handelt es sich hierbei um eine Verlagerung oder Erweitung der Aufgaben und welchen Stellenwert nehmen die Projekte im zukünftigen Tätigkeitsfeld ein? Diese Fragen gilt es im Folgenden zu klären.

Um über eine Verlagerung des Aufgabenfeldes des Forstsektors zu sprechen, sollen zunächst einige allgemeine Aussagen zu den integrativen Projekten die Einstellung der Forstämter in den verschiedenen Großstädten beleuchten. Die Forstverwaltung ist sich in urbanen Zentren darüber bewusst, dass sie einen Beitrag zur Lösung von allgemeinen sozialen Problemen leisten kann (I10). Diese Beiträge führen zu der Diskussion, bei welchen Aufgaben des Forstamtes es sich um Pflichtaufgaben und freiwillige Aufgaben handelt (I15). Klar herausgestellt wird auch, dass die Forstseite die eigene Zuständigkeit auf bestimmten Flächen erhalten will und so mit neuen Aufgaben konfrontiert ist (115). Das Bewusstsein der kommunalen Forstämter, dass eine Fülle von Angeboten aus dem Wald vorliegt, bringt diese in eine positive Position, sich mit den sozialen Problemen auseinander zusetzen (I25). 


\title{
5.7.1.4 Aufgabenerweiterung
}

Die allgemeinen, einleitenden Aussagen zeigen das veränderte Aufgabenverständnis der Forstverwaltung in Großstädten auf. Zu betrachten sind nun die Aussagen, die von den Forstämtern zum Bereich der Aufgabenerweiterung getätigt werden. Sie sollen zeigen, ob sozial integrative Projekte in den Aufgabenbereich aufgenommen werden oder bereits aufgenommen sind und als neue Dienstleistung verstanden werden. Das eigene Verständnis des Forstamtes, als Dienstleister zu fungieren, beschreibt das erste Beispiel. Hier wird der Wald als Landschaftsraum gesehen, in dem von eigener Seite eine personelle und technische Infrastruktur sichergestellt werden muss. Aber auch Dienstleistungen von der Umweltbildung bis hin zur Kunst können im Wald mit Unterstützung des Forstamtes angeboten werden (15). In den Gesprächen wird auch die Differenzierung der eigenen Aufgaben angesprochen. So sind Pflichtaufgaben zu leisten und freiwillige Aufgaben bedürfen eines offiziellen Auftrages und eines Budgets zur Durchführung. Kann aber für eine freiwillige Aufgabe die Wirtschaftlichkeit nachgewiesen werden, kann diese umgesetzt werden. Als Beispiel ist hier das „Hüttenwesen“ des Forstamtes Freiburg zu nennen, wobei es sich um die Vermietung von Grillhütten im Stadtwald handelt. Da dieser Bereich als wirtschaftlich zu bezeichnen ist, kann diese freiwillige Aufgabe umgesetzt werden (I15). Im Aufgabenverständnis einiger befragter Forstverwaltungen ist der Beitrag zur Lösung sozialer Probleme bereits verankert und wird als Anspruch der Gesellschaft an die eigene Institution gesehen.

\begin{abstract}
„Also ich will jetzt eigentlich da hin, dass wir gesagt haben, das ist eine Leistung, die man von uns eigentlich erwartet, weil es sind erhebliche soziale Probleme da und wenn wir mit unserem Spektrum der Aufgaben dazu ein kleines Stück Lösung beitragen können, dann müssen wir das eigentlich machen. Dann hat die Gesellschaft einen Anspruch darauf, dass wir solche Dienstleistungen zur Verfügung stellen." (I15)
\end{abstract}

Dies Beispiel macht deutlich, dass die Sozialfunktion des Waldes, über das klassische Verständnis (Erholung, Sport etc.) hinaus, als Dienstleistung an der Gesellschaft verstanden wird. Zum Aufgabenverständnis der Forstveraltung in Großstädten zählt auch, die Zuständigkeit für problematische Waldflächen zu erhalten, z.B. die Flächen, die sehr klein parzelliert sind. Hierzu bedarf es der Erschließung neuer Aufgaben, die auf diese Flächen zugeschnitten sind und in das Tätigkeitsfeld neu aufgenommen werden (I15). Das Angebot von kostengünstigen Lösungen für soziale Probleme ist ein Vorteil des Forstsektors (I15). Wo andere Institutionen nur mit erheblichem finanziellen Aufwand zu einer Integration von Problemgruppen beitragen können, liegt im Wald das Integrationspotential kostengünstiger vor. Hierzu ist es aber nötig, neue Dienstleistungen zu erarbeiten und anzubieten. Die aufgeführten Beispiele zeigen eine größtenteils bewusste Erweiterung der Aufgaben des Forstsektors, die den Ansprüchen der städtischen Bevölkerung entspricht. Bleibt nun zu klären, ob es sich bei der Aufgabenerweiterung um eine Momentaufnahme handelt, also eine 
einmalige Aktion zur Integration oder um ein zukunftsfähiges Aufgabengebiet. Aus den Angaben der Gesprächspartner zeichnet sich ab, dass die integrativen Projekte in das Tätigkeitsfeld der Forstämter aufgenommen werden sollen. So wird beschrieben, dass das Forstamt offen für neue Ideen sein sollte und diese in den Arbeitsablauf integrieren sollte (I5). Gerade die Beschäftigungsmaßnahmen werden aufgrund der Effektivität ein Bestandteil der forstlichen Aufgaben sein. Denn durch den Einsatz von Langzeitarbeitlosen auf den Flächen des Forstamtes können zusätzliche Aufgaben erfüllt werden, die mit dem eigenen Personal nicht geleistet werden können (I13, I21). Die Aufnahme der integrativen Projekte in den Tätigkeitsbereich hängt immer wieder von der Finanzierung der Projekte ab. Dieser Fall kann in Hamburg nachgewiesen werden, da die integrative Beschäftigungsmaßnahme wegen der fehlenden Finanzierung nicht fortgesetzt wurde (123). Um die Zukunft der Forstverwaltungen in den Großstädten zu sichern, muss nach eigenen Angaben (I25), eine Verlagerung bzw. eine Erweiterung der Aufgaben stattfinden, sonst verliert der Forstsektor an Bedeutung. So sollten Chancen, die ein neues Tätigkeitsfeld und Finanzierungsmöglichkeiten erschließen, genutzt werden, z.B. Programm „Soziale Stadt“. Zusammenfassend lässt sich festhalten, dass sich die kommunalen Forstveraltungen in urbanen Zentren über die Verlagerung des originären Aufgabenspektrums auf neue und angepasste Aufgaben durchaus bewusst sind. Die Aufnahme der sozialen Integration in das Tätigkeitsfeld, sollte langfristig angelegt sein und ist in den meisten Fällen eine Reaktion auf sich wandelnde gesellschaftliche Probleme. Alle befragten Forstämter verstehen sich als Dienstleister, die sich mit neuen Angeboten an die verlagerte Nachfrage anpassen. Diese Anpassung muss gerade im Forstbereich stattfinden, um die „Daseinsberechtigung“ (I15) aufrecht zu erhalten.

\subsection{Schlussbetrachtung der Hypothese III}

Hypothese III: a) Die Forstverwaltung kann durch die Vermarktung des Produktes „Integration“ Lösungen zur Nutzung des sozialen Potentials des Waldes bieten.

b) Die Forstverwaltung kann durch die Vermarktung des Produktes „Integration“ neue Ressourcen erschließen und den eigenen Handlungsspielraum erweitern.

Der Beitrag der kommunalen Forstverwaltung zur Nutzung des sozialen Potentials des Waldes durch Kooperation mit anderen Institutionen wurde aufgezeigt. Die Analyse zeigt, dass die Annahmen des ersten Teil der Hypothese III nicht bestätigt werden können. Die Lösungen zur Nutzung des sozialen Potentials des Waldes durch die Vermarktung des Produktes Integration können nicht nachgewiesen werden. Die Aufnahme von Integration in die Produktpalette der Forstverwaltung, ist annähernd in drei der untersuchten Fallstudien 
(Freiburg, Düsseldorf, Nürnberg) aufgedeckt worden. In Freiburg ist ein Beschäftigungsprojekt für Jugendliche in Zusammenarbeit mit einer Beschäftigungsfirma in das Leitbild der Forstverwaltung aufgenommen worden und mit einem Preis prämiert, was eine nachhaltige Wirkung auf die Aufgaben der Forstverwaltung hat (Fortführung von Beschäftigungsprojekten). In Düsseldorf hat das Garten-, Friedhofs- und Forstamt ein vierstufiges System von Arbeitsgelegenheiten erarbeitet, welches Menschen in den Arbeitsmarkt integrieren soll. Besonderes Merkmal dieser Arbeitsgelegenheiten ist die Langfristigkeit und die Übernahme von Personen in feste Anstellungen im eigenen Amt. In Nürnberg ist das Arbeitsprojekt für Suchtkranke im Wald auf die soziale Rehabilitation der Klientel angelegt. Auf Forstamtsflächen wird Holz eingeschlagen und als Brennholz durch die soziale Institution bzw. die Problemgruppen vermarktet. Diese drei Beispiele weisen auf eine Annäherung an die Produktgestaltung der Forstverwaltung hin, da diese Maßnahmen öffentlichkeitswirksam eingesetzt werden. Eine tiefe Durchdringung des Produktes „soziale Integration" scheint nur in den wenigsten der untersuchten Fälle geschehen zu sein. Integration bedeutet mehr als nur die zeitlich befristete Auseinandersetzung mit Problemgruppen. Die Hilfe zur Wiedereingliederung in die Gesellschaft muss langfristig angelegt sein, um nachhaltige Effekte zu erzielen. Dies kann von Seiten der Forstverwaltung nur geschehen, wenn sie das Produkt Integration auf langfristige Sicht anbieten kann. Eine Langfristigkeit konnte in den Bemühungen der Forstverwaltung nur in den drei o.g. Fällen festgestellt werden. Die Falsifizierung des ersten Teils der dritten Hypothese zieht die Falsifizierung der Möglichkeit, Ressourcen aus der Vermarktung des Produktes Integration zu erschließen, nach sich. Da Integration nicht als Produkt definiert wird, kann die Erschließung von finanziellen Ressourcen nicht stattfinden. Die Forstverwaltungen sind zum Zeitpunkt der Untersuchung nicht in der Lage, durch die Dienstleistung „Integration“ eine neue Einnahmequelle zu schaffen. Eher das Gegenteil ist der Fall. Einige Forstverwaltungen zahlen für integrative Beschäftigungsmaßnahmen im Wald (z.B. die Entfernung der Spätblühenden Traubenkirsche auf Forstamtsflächen). Auffällig ist auch, dass sich die Forstverwaltungen nicht an finanziell gut ausgestatteten Projekten beteiligen. Als Beispiel sei der Etat der Stadt Frankfurt in Höhe von 100.000 EURO für trägerübergreifende Integrationsprojekte angeführt. Hier wäre die Möglichkeit, finanzielle Ressourcen zu erschließen, gegeben, aber eine Beteiligung des städtischen Forstamtes fehlt gänzlich. Diese Nicht-Beteiligung zeigt eindringlich, dass es einer Hinwendung zu neuen Produkten bzw. Aufgaben und deren Finanzierungsmöglichkeiten von Seiten der Forstverwaltung bedarf.

Trotz des fehlenden Produktes „Integration“ hat die Forstverwaltung ihre Aufgaben um die Integration erweitert, auch wenn dies in einigen Fälle nur für einen begrenzten Zeitraum geschehen ist. So kann die Annahme der Erweiterung des Handlungsspielraumes auch in 
diesem Fall als bestätigt angesehen werden. Der Beitrag zur Lösung gesamtgesellschaftlicher Probleme, z.B. die Wiedereingliederung von Problemgruppen in den Arbeitsmarkt, zeigt einen Wandel in der Aufgabenerfüllung der Forstverwaltung. Durch die Bereitschaft, sich auf neue Aufgabenbereiche einzulassen, gewinnen die kommunalen Forstämter allgemein an Bedeutung in der städtischen Ämterstruktur. Die Analyse zeigt eine Veränderung des Selbstverständnisses im Forstsektor vom „Waldbewahrer“ zum „Dienstleister“ und damit einhergehend ein Wandel im Aufgabenverständnis.

\section{Fazit und Handlungsempfehlungen}

Die Ergebnisse des Forschungsprojektes zeigen zusammenfassend, dass der Forstsektor in der Lage ist, die Diskussion um die soziale Dimension von Nachhaltigkeit mitzugestalten. Die bisherige Definition des sechsten Kriteriums der Helsinki Kriterien „Erhaltung anderer sozioökonomischer Funktionen und Bedingungen" (Deutscher Forstwirtschaftsrat, 2006) reicht mit der Ausrichtung auf ökonomische Faktoren nicht aus, um soziale Nachhaltigkeit umfassend zu definieren. Indikatoren wie der Beitrag des Forstsektors zum Bruttoinlandsprodukt, die Anzahl der Arbeitskräfte im Sektor und die Zugänglichkeit des Waldes zum Zweck der Erholung (MCPFE, 2002) sind nur in Teilen geeignet, um die Funktion des Waldes für die soziale Nachhaltigkeit zu beschreiben. Durch die Projekte zur sozialen Integration erlangen die Forstverwaltungen Kompetenzen und Informationen, durch die sie in der Lage sind, einen entscheidenden Beitrag zur Definition von sozialer Nachhaltigkeit zu leisten, der nicht ungenutzt bleiben sollte.

Die vorliegende Arbeit beweist eine Nutzung des integrativen Potentials des Waldes. Es finden nachgewiesene Projekte zur sozialen Integration, mit dem Schwerpunkt der Beschäftigungsprojekte für verschiedene Problemgruppen, statt. In einigen der untersuchten Fallstudien bleibt dieses Potential aber auch ungenutzt, s. Frankfurt a.M.. Die Möglichkeit einer Finanzierung von integrativen, trägerübergreifenden Projekten durch die Stadt Frankfurt wird nicht in Anspruch genommen. Die häufigste Antwort auf die Nicht-Beteiligung ist die Personalknappheit, aufgrund derer die Integrationsprojekte mit der derzeitigen Personaldecke nicht zu leisten sind. Eine Überlegung wäre hier, die Projekte mit Honorarkräften, die extern angeworben werden, durchzuführen. Diese Strategie würde dazu führen, dass das integrative Potential nicht ungenutzt bliebe oder in eine andere Zuständigkeit übergeht. Das Forstamt wird so mit relativ wenig Aufwand, eben durch die Bereitstellung von Flächen, positiv in das Licht der Öffentlichkeit gerückt. Wenn Personal und 
Kompetenz für Projekte dieser Art fehlen, sollten sich die Forstbehörden genau diese Faktoren von außen zur Hilfe holen und so einen Beitrag zur sozialen Integration leisten.

Bei der Analyse der Ergebnisse fällt auf, dass die Institutionen des sozialen Sektors die Merkmale von erfolgreichen integrativen Maßnahmen besser und schneller definieren können als die Vertreter der Forstämter. Es liegt auf der Hand, dass Institutionen, die sich schwerpunktmäßig mit integrativen Projekten auseinandersetzen, Faktoren geläufig sind, die zum erfolgreichen Abschluss der Projekte beitragen. Ist die Forstverwaltung durch die Kooperation mit diesen Institutionen in der Lage, einen Gewinn von Kenntnissen und Informationen über das Klientel und die Erfolgfaktoren von Integrationsprojekten zu erzielen, kann sie als kompetenter Partner auftreten und sich in zukünftige Projekte aktiv einbringen. Zur Zeit findet die aktive Einbringung eher selten statt, so dass ein Beitrag zur Lösung gesellschaftlicher Probleme nur zeitlich und effektiv eingeschränkt stattfinden kann.

Die Analyse des Datenmaterials weist eine Umweltbildungsmaßname aus, die eine Nutzungsgebühr der Erholungseinrichtungen nach sich zieht. Ein solches System könnte auch für die Leistung von Integration angedacht werden. Besitzt die Forstverwaltung Kompetenzen in Bezug auf die Integration von Problemgruppen, könnte in einem möglichen Projekt die Forstverwaltung einen Mitarbeiter für diese Arbeiten abstellen, dessen Arbeitseinsatz von der Organisation bezahlt würde. Das bedeutet, die Forstverwaltung "verkauft“ die zuvor erworbene Kompetenz sozialer Nachhaltigkeit an nachfragende Organisationen. Zudem kann das Wissen über den Wald und die Arbeiten im Wald der Forstverwaltung von den sozialen Institutionen genutzt werden. Gerade in Beschäftigungsmaßnahmen kann die Forstseite Informationen liefern über die Schwere der Arbeiten, sie kann die Arbeiten als sinnvoll einschätzen und kann lenkend wirken, falls Arbeiten geplant werden, die den Tätigkeitsbereich des forstlichen Personals beschneiden. Dieses Wissen und die Informationen können sich demnach die sozialen Institutionen zu Nutze machen und daraufhin die Planung von Projekten effektiver gestalten. Hier kann von einer klassischen win-win-Situation auf beiden beteiligten Seiten gesprochen werden.

Die gemeinschaftlichen Integrationsprojekte weisen neben dem Effekt für die teilnehmenden Personen und den beteiligten Institutionen auch einen Nutzen für die gesamte Gesellschaft auf. Ein Zitat soll dies verdeutlichen:

„Gut, der Nutzen gesamt für die Stadt Frankfurt ist sicherlich im sozialen Bereich zu sehen, dass Leute, die irgendwo eine Beschäftigung haben besser integriert sind in die Gesellschaft als Leute, die zu Hause sitzen und nichts zu tun haben. Also das ist der Gesamtnutzen, ich denke mal, für die Gesellschaft." (I20)

Der Gesamtnutzen der sich für die Gesellschaft gerade aus Beschäftigungsprojekten ergibt, besteht sicherlich zunächst in der Möglichkeit, über Arbeit eine verbesserte Positionierung im gesellschaftlichen Leben zu erreichen und so integriert zu sein. Der Gesamtnutzen besteht zum anderen aber auch in dem Ziel, langzeitarbeitslose Personen arbeitsfähig zu halten. 
Nach Arbeitslosigkeit, die schon einige Jahre anhält, sind die Menschen nicht mehr in der Lage, körperlich anspruchsvolle Arbeit, wie z.B. Waldarbeit zu verrichten. Außerdem belaufen sich die gesamtfiskalischen Kosten der Arbeitslosigkeit im Jahr 2004 aus insgesamt 86 Mrd. EURO, die sich zu 54\% aus gezahltem Arbeitslosengeld und Arbeitslosenhilfe und zu 46\% aus Mindereinnahmen von Steuern und Sozialbeiträgen zusammensetzen (Institut für Arbeitmarkt und Berufsforschung, 2007). Diese Zahlen stehen für die Belastung der öffentlichen Haushalte von Bund, Länder und Gemeinden auf der Einnahmen- und Ausgabenseite sowie die Kassen der Versicherungsträger. Das Entgegenwirken der finanziellen Belastung soll Ziel der Beschäftigungsmaßnahmen sein und ist definitiv als Gesamtnutzen für die Gesellschaft zu bewerten. Könnten die Kooperationsprojekte mit Waldbezug einen Beitrag zur Senkung der Kosten leisten, wäre der politische Einfluss der Forstverwaltungen, gerade in urbanen Zentren, nicht wegzudiskutieren. Dies kann selbstverständlich nur dann geschehen, wenn durch die Vermittlung von Fähigkeiten und Kenntnisse in den Beschäftigungsprojekten mit Waldbezug Arbeitsverhältnisse entstehen, sei es auf dem ersten oder dem zweiten Arbeitsmarkt. Auch die Projekte für abhängigkeitserkrankte Menschen, seien es Beschäftigungsprojekte oder direkte therapeutische Maßnahmen, mit Waldbezug tragen zu einen gesamtgesellschaftlichen Nutzen bei. Die laufenden Gesundheitsausgaben in Deutschland im Jahr 2004 beliefen sich auf ca. 225 Mrd. ERUO, von denen ca. 9 Mrd. EURO auf Maßnahmen zur „Prävention“ und dem "Gesundheitsschutz" und 12,5 Mrd. EURO auf „therapeutische Leistungen“ entfielen (Gesundheitsberichterstattung des Bundes, 2007). Auch hier könnte angedacht werden, dass die Projekte zur Rehabilitation von Suchtkranken im Wald die Gesundheitsausgaben für diese Klientel senken könnten. Über die billigere Bereitstellung von sozialen Leistungen wurde im Fallbeispiel Freiburg berichtet und dies könnte einen entscheidenden politischen Einfluss der Forstverwaltung nach sich ziehen. Diese Möglichkeit wird aber von Seiten der Forstverwaltungen nicht genutzt, was mit einem eingeschränkten Aufgabenverständnis zu erklären ist.

Die Arbeiten im Wald, die von Gruppen mit erheblichen Problemen durchgeführt werden können, sind nicht sehr abwechselungsreich und senken so die Motivation der Teilnehmer. Um die Arbeiten interessanter zu gestalten, müssten Arbeitsbereiche einbezogen werden, die den Aufgabenbereich des forsteigenen Fachpersonals beschneiden würden. (vgl. Kap.

„Aber das ist ja genau die Problematik, man könnte das sicherlich viel interessanter gestalten, aber dann wären wir sofort im originären Aufgabenbereich der Forstwirte, wenn man sagt, jetzt mäht ihr mal hier oder macht mal da die Bank oder sonst was, was sie mit Sicherheit auch gerne gemacht hätten und wo man auch nicht immer gleich eine qualifizierte Ausbildung für haben muss, aber da kommen wir wirklich in einen großen Konflikt." (I23) 
In der Gesamtbetrachtung der Untersuchung sollten deshalb die kritischen Äußerungen zu diesen Maßnahmen nochmals aufgegriffen werden. In einigen der untersuchten Fallstudien wurde der Einsatz der Klientel der Beschäftigungsfirmen sehr kritisch gesehen. Hier wurde eine Beschneidung des originären Tätigkeitsbereichs des amtseigenen Personals befürchtet. Bevor es in diesen Fällen (v.a. Hamburg) zu einer Kooperation zwischen Forst und Beschäftigungsfirma kam, wurden die Arbeitseinsätze eingehend geprüft und diskutiert. Konnten die Bedenken der Forstseite ausgeräumt werden, stand den effektiven Arbeitsprojekten nichts mehr im Wege. Die Befürchtungen der Forstseite sind durchaus berechtigt, denn eine Studie von Kettner und Rebien (2007) belegt den Einsatz von Zusatzjobbern (ALG II Empfängern) in Betrieben, die eigentliche Tätigkeiten der regulären Belegschaft übernehmen. Dies kann soweit gehen, dass die Zusatzjobs zum Personalabbau benutzt werden, denn der Einsatz von öffentlich finanzierten Beschäftigten rechnet sich für einen Betrieb besser als die Beschäftigung eines/r regulär sozialversicherungspflichtigen Arbeitnehmers/in (Kettner und Rebien, 2007, S. 61).

Weiterhin wurde in den Gesprächen mit den Experten Kritik an Beschäftigungsmaßnahmen laut, die nach dem Sinn der Projekte im Forstbereich fragt, obwohl dort keine Perspektive geboten werden kann. Die Perspektive wird hier in einer Übernahme in ein Beschäftigungsverhältnis nach Absolvierung einer Qualifizierungsmaßnahme gesehen. Die Hauptaufgabe der Waldarbeit, nämlich die Holzernte ist sicherlich nur von ausgebildetem Personal durchzuführen, dennoch lassen sich Arbeiten finden, die von ungelernten oder angelernten Kräften zu erfüllen sind (z.B. Freiräumen der Pflanzfläche). Aber die Übernahme in eine Festanstellung muss nicht ausdrückliches Ziel von Integrationsmaßnahmen sein. Die erlernten Fähigkeiten in der Waldarbeit bzw. in Teilbereichen der Waldarbeit qualifizieren die Personen, so dass eine erhöhte Chance in der Vermittlung in verwandte Fachbereiche besteht. Das Argument wäre hier, solange Beschäftigungsmaßnahmen im Wald die Personen qualifizieren und deren Kenntnisse erweitern, so dass die Aussichten auf eine Beschäftigung in einem beliebigem Bereich erhöht werden, sollten diese durchgeführt werden. Dieser Beitrag zur Sozialintegration kann als Kulturation in Verbindung mit der Plazierung, der verbesserten Position in der Gesellschaft, bezeichnet werden. Maßnahmen, die Interaktionen zwischen Menschen oder die Identifikation mit dem System fördern, können von der Forstverwaltung durchgeführt werden. Der Wald stellt einen günstigen Interaktionsraum zur Verfügung, in dem sich Menschen begegnen und Beziehungen untereinander aufbauen können. Die Identifikation mit dem Wald kann ebenfalls von der Forstverwaltung geleistet werden, ein Beispiel sind die Waldfeste, die von der Forstverwaltung für die Bewohner eines Stadtteils veranstaltet werden.

Den Forstverwaltungen ist weiterhin die ökonomische Waldfunktion wichtig, denn sie spielt für die Städte eine wesentliche Rolle in der Haushaltsplanung. Diese ökonomische 
Argumentation könnte sich die Forstverwaltung zu Nutze machen um integrative Projekte umzusetzen. Denn kann vorgelegt werden, dass die sozialen Leistungen zur Integration kostengünstiger von der Forstseite angeboten werden könnten, hätte dies einen entscheidenden städtepolitischen Einfluss und das Amt würde an Bedeutung gewinnen (Bsp. Freiburg: die Erholungsleistungen des Forstamtes können kostensparender angeboten werden, als die des „Amtes der städtischen Bäder“). Mit der ökonomischen Bilanz ist dem Forstsektor ein treffendes Argument zur Durchsetzung der Projekte in die Hände gelegt worden. Ein Vorschlag zur Eröffnung einer finanziellen Ressource stellt die Flächenbereitstellung dar. Bietet die Forstverwaltung „Integration“ nicht selbständig an, kann sie Flächen für eine soziale Institution und deren Integrationsmaßnahmen ausweisen. Die Ertragsverluste, die als Folge der Abweichungen von der betriebswirtschaftlichen optimalen Waldbewirtschaftung entstehen, müssten durch ein Bewertungskonzept ermittelt werden und von den Nutzern der Flächen ausgeglichen werden (vgl. Möhring, Rüping, 2006).

Weitere Handlungsempfehlungen, die sich aus der Analyse des Datenmaterials für die Forstverwaltungen ergeben, sind die Reaktion auf die Nachfrage integrativer Kooperationsprojekten und diese aktiv zu forcieren, um das Handlungsfeld, den Aufgabenbereich und die Bedeutung der Institution zu erweitern und zu erhöhen. Die Forstverwaltung darf sich vor den sich wandelnden Ansprüchen einer sich ebenfalls wandelnden Gesellschaft nicht verschließen. Zudem sollten Kompetenzen und Wissen in den Kooperationsprojekten wechselseitig ausgetauscht werden und auch die Einstellung von vielleicht fachfremden Fachleuten, wie z.B. Sozialarbeitern in Betracht gezogen werden, wenn dies einer angepassten Aufgabenerfüllung dienlich ist.

Die Forstverwaltungen in Großstädten müssen auf die sich wandelnden Ansprüche der Gesellschaft reagieren und ihre soziale Verantwortung gegenüber einer sich ebenfalls verändernden Gesellschaft übernehmen. Einige Bevölkerungsteile sind immer häufiger auf Hilfesysteme angewiesen und die Forstverwaltungen sind verpflichtet, einen Beitrag zu diesen Hilfesystemen zu leisten 


\section{Zusammenfassung}

In Deutschland ist das Thema Integration ein immer wiederkehrender Schwerpunkt in der politischen Diskussion. Die Lösung dieser gesamtgesellschaftlichen Aufgabe ist auf die Mitarbeit unterschiedlichster Sektoren angewiesen. Vor allem die großen Kommunen im Bundesgebiet sind von den Kosten der verschiedenen Integrationsleistungen besonders betroffen. Der Fokus der vorliegenden Arbeit ist auf diese urbanen Zentren gelegt, da sich hier ein Großteil der Bevölkerung angesiedelt hat und soziale Probleme hier kumulieren. Die Ansprüche, die in diesen Ballungszentren an den Wald und die Grünflächen gestellt werden, sind von einer Vielzahl und Konflikthaftigkeit gekennzeichnet. Die Aufgabe, diese Ansprüche zu erfüllen, obliegt den ansässigen Forstverwaltungen. Die Entwicklungen der letzten Jahre lassen eine abnehmende Tendenz der Bedeutung und des Einflussbereiches der Forstverwaltungen erkennen. Durch die immensen Haushaltsdefizite der Stadtverwaltungen ist ein Bürokratieumbau und -abbau in den Großstädten zunehmend an der Tagesordnung (Pelz, 1996). Von diesem Um- und Abbau von Verwaltungseinheiten bleibt die Forstverwaltung nicht verschont. Neben dem Bedeutungsverlust der Forstverwaltung in den Städten fällt in der Diskussion um die Nachhaltigkeit des Waldes ein Defizit in der Definition von "sozialer Nachhaltigkeit“ auf. Die Forstwirtschaft scheint nicht in der Lage zu sein, soziale Nachhaltigkeit über die Arbeitsplätze in der Forstwirtschaft und den Beitrag zum BIP hinaus zu interpretieren und zu definieren. Die Definition von sozialer Nachhaltigkeit umfasst neben der Chancengleichheit bezüglich der Ressourcenverteilung und der Geschlechter, der Partizipation (Empacher u. Wehling, 2002) auch die Integration als Anerkennung kultureller Unterschiede statt Ausgrenzung und die Vernetzung (Bundesministerium für Gesundheit und Soziale Sicherung, 2005). Da ein sozialer Wandel in der Gesellschaft stattgefunden hat und immer mehr Menschen am Rande dieser stehen, bedarf es Hilfesystemen, die eine Randständigkeit von einzelnen Personen und auch Personengruppen versuchen zu vermindern oder ganz zu verhindern. Diese Hilfesysteme werden in der Sozialarbeit in vielfältiger Form angeboten (Bäcker et al., 2000). Gerade in Großstädten, die ein breites Spektrum an gesellschaftlichen Gruppen auf engstem Raum vereinen, ist die Nachfrage nach Hilfesystemen sehr groß. Die sich anschließende Frage ist demnach: Können die Forstverwaltungen in urbanen Zentren einen Beitrag zur sozialen Integration leisten? Es ist davon auszugehen, dass die Forstverwaltungen dies nicht in Eigenleistung erbringen können. Daher ist zu klären, mit welchen Instrumenten und Strategien die Forstverwaltung das soziale Potential erschließt.

Die theoretische Grundlage dieser Untersuchung bildet zum einen das „Liberale Modell der sozialen Integration“ nach Fuchs (1999). Dieses Modell basiert auf den drei Ebenen des 
demokratischen Systems Kultur (Werte), Struktur (Regeln) und Prozess (Handlungen) (Fuchs 1999, S.165). In dieser Untersuchung gilt das Hauptinteresse der Prozessebene, die sich auf Handlungen bezieht, die zur Solidarität mit den Anderen und der Tolerierung der Anderen beitragen. Weiterhin stützt sich das Forschungsprojekt auf die vier Formen der Sozialintegration von Esser (2001): Kulturation, Plazierung, Interaktion und Identifikation. Das theoretische Grundgerüst der Untersuchung wird durch das Kooperative Verwaltungshandeln nach Benz (1994) ergänzt, weil davon auszugehen ist, dass die Forstverwaltung das integrative Potential des Waldes nicht ohne Hilfe erschließen kann. Kooperation zwischen Institutionen kann als Strategieentscheidung bezeichnet werden und hat die gemeinsame Aufgabenerfüllung bzw. Problemlösung zum Ziel. Der Lösungsansatz der Forstverwaltung, das soziale Potential des Waldes durch Kooperation zu erschließen, kann ergänzt werden. Die Entwicklung eines neuen, marktfähigen Produktes kann einen weiteren Lösungsansatz darstellen. Mantau (2001a,b) beschreibt die Produktlücke, die im Forstsektor besteht, da die Waldfunktionen als öffentliches Gut kaum in ein marktfähiges Produkt umgewandelt werden können. Die theoretischen Ausführungen lassen die Spezifizierung der Fragestellung zu: Kann die Forstverwaltung das soziale Potential des Waldes in Kooperation mit anderen Institutionen oder durch die Entwicklung eines neuen Produktes erschließen und so den eigenen Handlungsspielraum erweitern.

Die theoretischen Fragestellungen werden daraufhin durch leitfadengestützte Experteninterviews in acht ausgewählten Großstädten Deutschlands (Göttingen, Kassel, Freiburg i.Br., Frankfurt a.M., Hamburg, Düsseldorf, Berlin und Nürnberg) empirisch getestet. Die Auswahl der Experten erfolgte über die Institutionen, die an Projekten zur Integration im Wald beteiligt waren. Diese Experten entstammen vornehmlich dem forstlichen und sozialen Sektor. Die gewonnenen Daten wurden einer qualitativen Inhaltsanalyse unterzogen, deren Hauptelement das theoriegeleitete Kategoriensystem darstellt. Zur Auswertung wurde das Auswertungsprogramm MAXQDA herangezogen.

Die Analyse des Datenmaterials hat ergeben, dass die Forstverwaltung durchaus in der Lage ist, das soziale Potential des Waldes zu erschließen. Die Beteiligung an Projekten, die eine Integration von sozialen Problemgruppen zu Ziel haben, konnte nachgewiesen werden. Das eingeschränkte Verständnis von sozialer Nachhaltigkeit lässt den Beitrag der Forstverwaltungen zu diesen Projekten jedoch sehr gering ausfallen. Dieser Beitrag schlägt sich zumeist in der Bereitstellung von Flächen für Beschäftigungsmaßnahmen nieder. Gerade diese Beschäftigungsprojekte machen einen Großteil der Integrationsprojekte aus, deren ausgewiesenes Ziel in der Qualifizierung der Teilnehmer (Jugendliche, 
Langzeitarbeitslose, Migranten, Suchtkranke, Straffällige) und der Integration in den Arbeitsmarkt liegt.

Die weitere Analyse der Daten hat gezeigt, dass die Forstverwaltungen das soziale Potential des Waldes nur in Kooperation mit Institutionen des sozialen Sektors erschließen können. Diese Strategieentscheidung der Forstverwaltungen zieht eine Erweiterung des Handlungsspielraumes nach sich, da durch die Zusammenarbeit Kenntnisse und Informationen gewonnen werden, die in die Lösung von gesellschaftlichen Problemen eingebracht werden können. Der größte Nutzen, den die Forstverwaltung aus den Kooperationen mit sozialen Institutionen zieht, liegt in der Positionssteigerung des eigenen Amtes innerhalb der Ämterstruktur der Stadt und der Öffentlichkeit.

Eine zweite Handlungsstrategie der Forstverwaltung wird in der Entwicklung eines neuen Produktes „Integration“ gesehen, um das soziale Potential des Waldes zu erschließen. Dies kann, nach der Analyse der Daten, als nicht erwiesen betrachtet werden. Die Forstverwaltung ist nur eingeschränkt in der Lage, ein Produkt aus dem sozialen Potential des Waldes anzubieten, was sich in drei der untersuchten Fallstudien in einem längerfristigen Angebot von Integrationsprojekten niederschlägt. Die Forstverwaltung erschließt demnach keine finanziellen Ressourcen mit dem Angebot der Integration. Eher das Gegenteil ist der Fall, denn die Forstverwaltungen zahlen für die Beschäftigungsprojekte auf den amtseigenen Flächen. Eine Hinwendung zu neuen Produkten und Dienstleistungen ist bei einem sich wandelnden Aufgabenverständnis der Forstverwaltungen zu erkennen, bedarf dennoch einer Erweiterung auf soziale Problembereiche. Die Bedeutung des sozialen Potentials des Waldes sollte die Forstverwaltung noch stärker durchdringen. So ist es nicht unbedingt notwendig, einem Langzeitarbeitslosen einen Arbeitsplatz im Wald zu verschaffen. Schon die Vermittlung von Kenntnissen und Fertigkeiten durch die Waldarbeit kann der Person eine bessere Positionierung auf dem Arbeitsmarkt ermöglichen und folglich die soziale Integration positiv beeinflussen. 


\section{Literaturverzeichnis}

aid infodienst Verbraucherschutz, Ernährung und Landwirtschaft (2006) Hrsg.: Leitfaden "Innovative Waldprodukte“ Neue Einkommensquellen durch Vermarktung der Schutzund Erholungsleistungen on Wäldern. 1534/2006

Bäcker, G., Bispinck, R., Hofemann, K., Naegele, G. (2000): Sozialpolitik und soziale Lage in Deutschland, Band 2: Gesundheit und Gesundheitssystem, Familie, Alter, Soziale Dienste. 3. überarb. und erweit. Auflage, Westdeutscher Verlag, Wiesbaden

Benz, A. (1994): Kooperative Verwaltung: Funktionen, Voraussetzungen und Folgen. 1. Aufl., Baden-Baden, Nomos Verlagsgesellschaft

Bittner, A. (2003): Außerschulische Umweltbildung in der Evaluation: Wirkungen kurzzeitpädagogischer Maßnahmen auf Umwelt- und Naturschutzinteressen von Schülerinnen und Schülern der Sekundarstufe I. Kovač, Hamburg

Bogner, A, Menz, W. (2002a): Expertenwissen und Forschungspraxis: die modernisierungstheoretische und die methodische Debatte um die Experten. In: Bogner, A, Littig, B., Menz, W. (Hrsg.): Das Experteninterview - Theorie, Methode, Anwendung. Leske + Budrich, Opladen, S. 7-29

Bogner, A, Menz, W. (2002b): Das theoriegenerierende Experteninterview Erkenntnisinteresse Wissensformen, Interaktion. In: Bogner, A, Littig, B., Menz, W. (Hrsg.): Das Experteninterview - Theorie, Methode, Anwendung. Leske + Budrich, Opladen, S. 33-70

Bolte, K.M., Hradil, S. (1988): Soziale Ungleichheit in der Bundesrepublik Deutschland. 6. Aufl., Leske und Budrich, Opladen

Braun, A. (2000): Wahrnehmung von Wald und Natur. Leske + Budrich, Opladen

Bundesministerium für Gesundheit und Soziale Sicherung (2005a): 2. Armuts- und Reichtumsbericht der Bundesregierung: Lebenslagen in Deutschland. http://www.bmas.bund.de/BMAS/Redaktion/Pdf/Publikationen/lebenslagen-indeutschland-anhaenge-2005, property=pdf, bereich=bmas, sprache=de, $r w b=$ true.. df (22.03.2006)

Bundesministerium für Gesundheit und Soziale Sicherung (2005b): Nachhaltige Entwicklung. http://www.bmgs.bund.de/deu/gra/themen/sicherheit/nachhaltigkeit/index.php (03.11.2005)

Bundesregierung 2006: Nationaler Integrationsplan. http://www.bundesregierung.de (13.12.2006)

Bundestransferstelle Soziale Stadt (2007): Bund-Länder-Programm "Soziale Stadt“. http://www.sozialestadt.de/programm/ (22.01.2007)

Burgbacher, H. (2001): Waldkonvention - Zielsetzungen, Grundsätze der Waldwirtschaft und Betriebsführung im Stadtwald Freiburg.

Burgbacher, H. (1996): Der Stadtwald Freiburg. AFZ/Der Wald 20/1996, S. 1094-1099 
Chassé, K. (1992): Brauchen wir den Randgruppenbegriff? In: Cassé, K., Drygala, A., Schmidt-Noerr, A. (Hg): Randgruppen 2000 - Analysen zu Randgruppen und zur Randgruppenarbeit. Böllert, KT-Verlag, Bielefeld

Deutscher Forstwirtschaftsrat (2006): Nachhaltige Waldbewirtschaftung in Deutschland. http://www.dfwr.de/broschuere de/inhalt.htm (März 2006)

Deutscher Städtetag (2004): Mitglieder des Deutschen Städtetages. www.staedtetag.de (25.05.2004)

Diekmann, A. (2000): Empirische Sozialforschung - Grundlagen, Methoden, Anwendungen. Rowohlts Enzyklopädie, Hamburg

Dieterich, V. (1953): Forstwirtschaftspolitik - Eine Einführung. Verlag Paul Parey, Hamburg, Berlin

Eekhoff, J., Roth, S.J. (2002): Brachliegende Fähigkeiten nutzen, Chancen für Arbeitslose verbessern. Stiftung Marktwirtschaft, Berlin

Empacher, C., Wehling, P. (2002): Soziale Dimension von Nachhaltigkeit - Theoretische Grundlagen und Indikatoren. ISOE - Studientexte, Nr. 11, Frankfurt a.M.

Esser, H. (2000): Soziologie Spezielle Grundlagen. Band 2: Die Konstruktion der Gesellschaft. Campus Verlag, Frankfurt a.M.

Esser, H. (2001): Integration und ethnische Schichtung. Arbeitspapiere - Mannheimer Zentrum für Europäische Sozialforschung, Nr. 40

Friedrich Ebert Stiftung (2006): Gesellschaft im Reformprozess. http://www.fes.de/aktuell/documents/061017 Gesellschaft im Reformprozess komplett. pdf (28.03.2007)

Fuchs, D. (1999): Soziale Integration und politische Institutionen in modernen Gesellschaften. In: Friedrichs, J., Jagodzinkski, W., (Hrsg.): Soziale Integration. Kölner Zeitschrift für Soziologie und Sozialpsychologie, Sonderheft 39/1999, Westdeutscher Verlag GmbH, Opladen/Wiesbaden, S.147-178

Galuske, M. (2005): Methoden der sozialen Arbeit - Eine Einführung. 6. Auflage, Juventa Verlag, Weinheim und München

Garten-, Friedhofs- und Forstamt Düsseldorf (2005): Informationsvorlage, Ö Vorlagen - Nr. $70 / 3 / 2005$

Georg-Ludwig-Hartig-Stiftung (2007): Begriff der Nachhaltigkeit. http://www.glhartig.de/nachhaltigkeit/index.htm (12.03.2007)

Germann-Chiari, C., Seeland, K. (2004): Are urban green spaces optimally distributed to act as placed for social integration? Results of a geographical information system (GIS) approach for urban forestry research. Forest Policy and Economics 6 (2004), 3-13

Gesundheitsberichterstattung des Bundes (2007): Gesundheitsausgaben in Deutschland. http://www.gbe-bund.de (03.04.2007) 
Ginsberg, L. (1996): Understanding Social Problems, Policies, and Programs. $2^{\text {nd }}$ ed. University of South Carolina Press

Glatzer, W. (2004) unter Mitarbeit von Krätschmer-Hahn, R.: Integration und Partizipation junger Ausländer vor dem Hintergrund ethnischer und kultureller Identifikation Ergebnisse des Integrationssurveys des BiB. Materialien zur Bevölkerungswissenschaften Heft 105c, Bundesinstitut für Bevölkerungsforschung beim Statistischen Bundesamt, Wiesbaden

Gouder, D., Neureuter, A., Roesler, M., Bühring, W. (2003): Ich - Draußen - Ein Naturerlebnisprojekt für Gruppen und Teams. ökom Verlag, München

Hafeneger, B., Schröder, A. (2005): Jugendarbeit. In: Otto, H.-U., Thiersch, H. (Hg.): Handbuch Sozialarbeit Sozialpädagogik. 3. Auflage, Ernst Reinhardt Verlag, München, S. 840-850

Hildenbrand, B. (1995): Fallrekonstruktive Forschung. In: Flick, U., v. Kardorff, E., Keupp, H., v. Rosenstiel, L., Wolff, S. (Hrsg.): Handbuch Qualitative Sozialforschung: Grundlagen, Konzepte, Methoden und Anwendungen. 2. Aufl., Beltz Psychologie Verlags Union, Weinheim

Institut für Arbeitsmarkt- und Berufsforschung (2007): Kosten der Arbeitslosigkeit Gesamtfiskalische Kosten der Arbeitslosigkeit 2004. http://iab.de/iab/aktuell/info kostenalo.htm (03.04.2007)

Jungblut, H.-J. (2005): Drogenpolitik. In: Otto, H.-U., Thiersch, H. (Hg.): Handbuch Sozialarbeit Sozialpädagogik. 3. Auflage, Ernst Reinhardt Verlag, München, S. 275-300

Kettner, A., Rebien, M. (2007): Soziale Arbeitsgelegenheiten. Einsatz und Wirkungsweise aus betrieblicher und arbeitsmarktpolitischer Perspektive. IAB Forschungsbericht Nr. $2 / 2007$

Keupp, H. (1976): Abweichung und Altagsroutine - Die Labeling-Perspektive in Theorie und Praxis. Hoffman und Campe Verlag, Hamburg

Killich, S. (2005): Kooperationsformen. In: Becker, T. (Hrsg.): Netzwerkmanagement - Mit Kooperation zum Unternehmenserfolg, Springer Verlag, Berlin, S. 13-22

Knoblauch, H. (2006): Transkription. In: Bohnsack, R., Marotzki, W., Meuser, M. (Hrsg.): Hauptbegriffe Qualitativer Sozialforschung. 2. Aufl., Verlag Barbara Budrich, Opladen \& Farmington Hills, S. $159 f$.

Konijnendijk, C. C. (2000): Die wachsende Bedeutung von „Urban Forestry“ in Europa. AFZ/Der Wald 11/2000, S. 575-576

Kreckel, R. (2005): Soziale Ungleichheit. In: Otto, H.-U., Thiersch, H. (Hg.): Handbuch Sozialarbeit Sozialpädagogik. 3. Auflage, Ernst Reinhardt Verlag, München, S. 17291735

Kromrey, H (1994): Empirische Sozialforschung. 6. Aufl., Leske und Budrich, Opladen 
Krott M. (1990): Öffentliche Verwaltung im Umweltschutz - Ergebnisse einer behördenorientierten Policy-Analyse am Beispiel Waldschutz. Studienreihe Konfliktforschung; 5, Braumüller, Wien

Krott, M. (1998): Urban Forestry: Management within the focus of people and trees. In: Krott, M., Nilsson, K. (Eds.): Urban Forestry - Multiple-Use of town forests in international comparison. $1^{\text {st }}$ Forum on Urban Forestry, Working Group S.6.14.00, Wuppertal, S. 9-19

Krott, M., (2001): Politikfeldanalyse Forstwirtschaft - Eine Einführung für Studium und Praxis. Parey Buchverlag im Blackwell Wissenschafts-Verlag, Berlin - Wien

Kuckartz, U. (2001): Einführung in das Textanalysesystem MAXQDA. Berlin

Küker, S. (2002): Kooperation und Nachhaltigkeit - Ein prozessorientierter Gestaltungsansatz für eine Analyse der Beiträge von Kooperation zum nachhaltigen Wirtschaften. Verlag Dr. Kovač, Hamburg

Lamnek, S. (2005): Qualitative Sozialforschung: Lehrbuch. 4. Aufl. Beltz Verlag, Weinheim, Basel

Leibundgut, H. (1975): Wirkungen des Waldes auf die Umwelt des Menschen. Eugen Rentsch Verlag, Erlennach-Zürich, Stuttgart

Lijphart, A. (1971): Comparative Politics and the Comparative Method. The American Political Science Review, Vol. 65, No. 3. (Sep., 1971), pp. 682-693

Lockwood, D. (1979): Soziale Integration und Systemintegration. In: Zapf, W. (Hrsg.): Theorien des sozialen Wandels. 4. Aufl., Königstein/Ts., S. 124-137

Mantau, U. (2001a): Die forstliche Produktlücke. In: Mantau, U. (Hrsg.): Beiträge zur Vermarktung der Umwelt- und Erholungsleistungen des Waldes. Sonderveröffentlichung von AFZ Der Wald,

Mantau, U. (2001b): Von der Waldfunktionenlehre zur Waldproduktlehre. In: Mantau, U. (Hrsg.): Beiträge zur Vermarktung der Umwelt- und Erholungsleistungen des Waldes. Sonderveröffentlichung von AFZ Der Wald,

Marotzki, W. (2006): Leitfadeninterview. In: Bohnsack, R., Marotzki, W., Meuser, M. (Hrsg.): Hauptbegriffe Qualitativer Sozialforschung. 2. Aufl., Verlag Barbara Budrich, Opladen \& Farmington Hills, S. 114

Mayring, P. (1997): Qualitative Inhaltsanalyse. Grundlagen und Techniken. 6. Aufl. Deutscher Studien Verlag, Weinheim

MCPFE (1993): Resolution H1 - General Guidelines for the Sustainable Management of Forests in Europe. http://www.mcpfe.org/resolutions/helsinki/resolution h1.pdf (12.03.2007)

MCPFE (2002): Improved Pan-European indicators for sustainable forest management. MCPFE Expert Level Meeting 7-8 October 2002, Vienna, Austria

Mertens, B. (2000): Absatzwege und Vertragskonzepte für forstliche Umwelt- und Erholungsprodukte: Schlussfolgerungen aus 98 Fallstudien vor dem Hintergrund des Transaktionskostenansatzes. Lang Verlag, Frankfurt a.M. 
Metzler, H., Wacker, E. (2005): Behinderung. In: Otto, H.-U., Thiersch, H. (Hg.): Handbuch Sozialarbeit Sozialpädagogik. 3. Auflage, Ernst Reinhardt Verlag, München, S. 118-139

Meuser, M. (2006): Inhaltsanalyse. In: Bohnsack, R., Marotzki, W., Meuser, M. (Hrsg.): Hauptbegriffe Qualitativer Sozialforschung. 2. Aufl., Verlag Barbara Budrich, Opladen \& Farmington Hills, S. 89ff.

Meuser, M., Nagel, U. (2002): ExpertInneninterviews - vielfach erprobt, wernig bedacht. Ein Beitrag zur qualitativen Methodendiskussion. In: Bogner, A, Littig, B., Menz, W. (Hrsg.): Das Experteninterview - Theorie, Methode, Anwendung. Leske + Budrich, Opladen, S. 71-93

Möhring, B., Rüping, U. (2006): Bewertungskonzept für forstliche Nutzungsbeschränkungen. Schriften zur Forstökonomie, J.D. Sauerländer's Verlag, Frankfurt a.M.

mudra (2006): mudra Jahresbericht 2006 / Tätigkeitsbericht 2005. www.mudraonline.de/archiv/jahresberichte/mudra ib2006.pdf (15.12.2005)

Mührel, E. (2005): Strafvollzug. In: Otto, H.-U., Thiersch, H. (Hg.): Handbuch Sozialarbeit Sozialpädagogik. 3. Auflage, Ernst Reinhardt Verlag, München, S. 1842-1849

Niedersächsisches Gesetz über den Wald und die Landschaftsordnung (NWaldLG). http://cdl.niedersachsen.de/blob/images/C8354368 L20.pdf

Niedersächsisches Ministerium für den Ländlichen Raum, Ernährung, Landwirtschaft und Verbraucherschutz (2007): Nachhaltigkeit - Verantwortung für die Zukunft. (http://www.ma.niedersachsen.de/master/C24943989 N25081077 l20 D0 I655.html (21.03.2007)

Ottitsch, A., Krott, M. (2005): Urban Forest Policy and Planning. In: Konijnendijk, C., Nielsson, K., Randrup, T. B., Schipperijn, J. (Eds.): Urban Forests and Trees. Springer Verlag, Berlin, Heidelberg, S. 117-148

Pelz, S. (1996): Ein kommunales Forstamt im Spannungsfeld der Verwaltungsreform. AFZ / Der Wald, 20/1996, S. 1100-1103

Randrup, T. B., Konijnendijk, C., Dobbertin, M. K., Prüller, R. (2005): The Concept of Urban Forstery in Europe. In: Konijnendijk, C., Nielsson, K., Randrup, T. B., Schipperijn, J. (Eds.): Urban Forests and Trees. Springer Verlag, Berlin, Heidelberg, S. 9-21

Schanz, H. (1996): Forstliche Nachhaltigkeit - Sozialwissenschaftliche Analyse der Begriffsinhalte und Funktionen. Dissertation. Schriften aus dem Institut für Forstökonomie der Universität Freiburg, Band 4

Simon, T. (2001): Wem gehört der öffentliche Raum. Leske+Budrich, Opladen

SGB II: Sozialgesetzbuch II - Grundsicherung für Arbeitssuchende - (Artikel 1 des Gesetzes vom 24. Dezember 2003, BGBI. I S. 2954), zuletzt geändert durch Artikel 2 des Gesetzes $\begin{array}{lllllll}\text { vom 20. } & \text { April } & 2007 & \text { (BGBI } & \text { I } & \text { S. }\end{array}$ http://bundesrecht.juris.de/bundesrecht/sgb 2/gesamt.pdf (28.05.2007) 
SGB III: Sozialgesetzbuch III - Arbeitsförderung - (Artikel 1 des Gesetzes vom 24. März 1997, BGBI. I S. 594) vom 24. März 1997 (BGBI. I S. 594, 595), zuletzt geändert durch Artikel 4 des Gesetzes vom 26. März 2007 (BGBI. I S. 378); http://bundesrecht.juris.de (14.03.2007)

SGB VIII: Sozialgesetzbuch VIII - Kinder- und Jugendhilfe (Artikel 1 des Gesetzes vom 26. Juni 1990, BGBI. I S. 1163) in der Fassung der bekanntmachung vom 14. Dezember 2006 (BGBI. I S. 3134), geändert durch Artikel 2 Abs. 23 des Gesetzes vom 19. Februar 2007 (BGBI. I S. 122). http://bundesrecht.juris.de/bundesrecht/sgb 8/gesamt.pdf (28.05.2007)

SGB XII: Sozialgesetzbuch XII - Sozialhilfe - (Artikel 1 des Gesetzes vom 27. Dezember 2003, BGBI. I S. 3022) zuletzt geändert durch Artikel 7 des Gesetzes vom 20. April 2007 (BBGI. I S. 554). http://bundesrecht.juris.de/bundesrecht/sgb 12/gesamt.pdf (28.05.2007)

Speidel, G. (1984): Forstliche Betriebswirtschaftslehre. 2. völlig neu bearb. Aufl., Parey, Hamburg - Berlin

Stadt Bonn (2006): Wie ist die Haushaltslage der Kommunen allgemein? http://www.bonn.de/rat verwaltung buergerdienste/buergermitwirkung/buergerhaushalt2 $\underline{006 / 00188 / i n d e x . h t m l ~(15.05 .2006) ~}$

Statistisches Bundesamt (2005): Statistik der Schwerbehinderten Menschen 2003. Kurzbericht, Wiesbaden. http://www.destatis.de/download/d/solei/schwerbbehinderte 03.pdf (03.04.2007)

StVollzG: Gesetz über den Vollzug der Freiheitsstrafe und der freiheitsentziehenden Maßregeln der Besserung und Sicherung - Strafvollzugsgesetz vom 16. März 1976 (BGBI. I S. 581, 2088), zuletzt geändert durch Artikel 2 Abs. 11 des Gesetzes vom 19. Februar 2007 (BGBI. I S. 122 mWv 1.1.2009); http://bundesrecht.juris.de

Tyrväinen, L., Pauleit, S., Seeland, K., de Vries, S. (2005): Benefits and Uses of Urban Forests and Trees. In: Konijnendijk, C., Nielsson, K., Randrup, T. B., Schipperijn, J. (Eds.): Urban Forests and Trees. Springer Verlag, Berlin, Heidelberg, S. 81-114

wfaa 2006: Werkstatt für angepasste Arbeit - Leitbilder. http://www.wfaa.de/index.php?id=3,0,0,1,0,0 (28.11.2006)

Wolf, A. (2005): Obdachlosigkeit. In: Otto, H.-U., Thiersch, H. (Hg.): Handbuch Sozialarbeit Sozialpädagogik. 3. Auflage, Ernst Reinhardt Verlag, München, S. 1292-1300

Wolffersdorff v., C. (2005): Drogen und Sucht. In: Otto, H.-U., Thiersch, H. (Hg.): Handbuch Sozialarbeit Sozialpädagogik. 3. Auflage, Ernst Reinhardt Verlag, München, S. 324-338

Wullschleger, E. (1982): Der Erfassung der Waldfunktionen. Berichte der eidgenössischen Anstalt für das forstliche Versuchswesen Birmensdorf, Nr. 238

http://www.e-geography.de/module/stadt 1/html/theorie 7.htm (Freie Universität Berlin) (20.08.2007) 
http://www.berlin.de (12.06.2007)

http://www.duesseldorf.de (12.06.2007)

http://www.frankfurt.de (12.06.2007)

http://www.freiburg.de (12.06.2007)

http://www.goettingen.de (12.06.2007)

http://www.hamburg.de (12.06.2007)

http://www.kassel.de (12.06.2007)

http://www.nuernberg.de (12.06.2007) 


\title{
9. Anhang
}

I. Interviewleitfaden - theoriegeleitet

Einleitung:

Thema kurz vorstellen, Sinn der Befragung

- Wieviel ha Waldfläche und Grünfläche bewirtschaftet das Forstamt?

- Können Sie in einigen Sätzen die Aufgaben ihres Amtes umreißen (Aufgabe, Zielgruppen)?

- Welche Waldfunktion steht in dem stadtnahen Wald im Vordergrund (Nutz-, Schutz- oder Erholungsfunktion)?

Hauptteil:

Definition:

- Würden Sie folgender Definition von sozialen Randgruppen zustimmen:

„Randgruppen vereinigen so gravierende Anhäufungen von sozialen Benachteiligungen auf sich, dass sie vom üblichen Leben in unserer Gesellschaft ausgeschlossen sind. Gründe für Randständigkeit: - Armut, Obdachlosigkeit

\author{
Betreuung, Haft \\ Isolation“ \\ Vorurteile, Diskriminierung,
}

- Würden Sie dieser Definition noch etwas hinzufügen wollen?

Kontakte und Verhältnis:

- Bestehen in Ihrem normalen Arbeitsalltag Berührungspunkte mit sozialen Randgruppen?

- Wie sehen diese Berührungspunkte aus

(Konflikt, Duldung)?

- Wie würden Sie die Randgruppen charakterisieren? Um welche Personengruppen handelt es sich?

- Wo ergeben sich in ihrer täglichen Arbeit Schwierigkeiten mit Problemgruppen?

- Mit welchen Mitteln wird versucht diese Probleme zu lösen?

(Instrumente: - Information

- Kooperation

- runde Tische

- finanzielle Maßnahmen etc.)

(Je nach Antwort)

- Ist eine Nutzung des Waldes durch Problemgruppen erwünscht?

- Welche Ansprüche haben soziale Randgruppen Ihrer Meinung nach an den Wald und die Grünflächen?

- Glauben Sie diese zu erfüllen? 
Maßnahmen:

„Der Begriff Integration bezieht sich aus soziologischer Sicht auf die verhaltens- und bewusstseinsmäßige Eingliederung und Angleichung von Personen oder Gruppen an Wertestrukturen und Verhaltensmuster."

- Finden sozial-integrative Maßnahmen im Wald oder auf Grünflächen ihres Zuständigkeitsbereich statt an denen sich Ihr Amt beteiligt?

- Langzeitarbeitslose?

Maßnahme?

Ziel

- Jugendliche (schwererziehbare, ausländische)?

- Behinderten - Projekte?

- „Soziale Stadt"?

- Suchtkranke?

- Straffällige?

- Wohnungslose?

- etc.

- Sind diese Projekte von längerer Dauer (z.B. jedes Jahr, jeden Monat, etc.) oder einmalige Aktionen?

- Werden diese Maßnahmen finanziell gefördert? Von wem?

- Werden sie für die Teilnahme an Projekten entschädigt? Wie?

(ökonomisch, Einflusspotentiale etc.)

- Stellt die Teilnahme an den Projekten eine Verlagerung ihrer Aufgaben dar? (Erschließung von Ressourcen, Aufgabenerweiterung, Personalentscheidungen?)

- Kann die Teilnahme an den Projekten auch zukünftig ein Aufgabengebiet darstellen?

- Was ist für Ihr Amt ein Erfolg für Integration?

- Können Sie eine erfolgreiche integrative Maßnahme beschreiben?

- Welche Merkmale charakterisieren eine solche Maßnahme?

Kooperation:

Finden zur Umsetzung der genannten Projekte zwischen Ihnen und anderen Institutionen Kooperationen statt?

- Wenn ja, mit wem und wie ist es zu diesem Kontakt gekommen?

- Wie sehr bringen sie sich in diese Zusammenarbeit ein (Personal, Zeit, Geld)?

- Wie ist die Zusammenarbeit der Partner strukturiert? (Wie würden Sie die Zusammenarbeit charakterisieren?)

(Gleichberechtigung, gleiche Verfahrens- und Entscheidungsrechte, Rechte und Pflichten)

- Wie läuft diese Zusammenarbeit praktisch ab?

(dialogische Kommunikation, „face - to - face" Kontakte, sprachliche Kommunikation über Ziele, Interessen, Problemdefinitionen, Situationseinschätzungen, Kenntnisse und Werthaltungen der Akteure mit der Absicht, diese aufeinander abzustimmen und in Einklang zu bringen)

- Wie würden Sie das Ergebnis der Zusammenarbeit beschreiben? (bzw. den Weg dorthin?) 
(freiwillige Einigung der beteiligten Akteure und Organisationen, Ziel ist die von allen Beteiligten akzeptierte Lösung der Probleme, Konkrete Problemlösung, gemeinsame Entscheidung, die von allen Beteiligten anerkannt wird (nur dann spricht man von kooperativem Verwaltungshandeln))

(o.g. Punkte $=$ Mehrdimensionalität von Kooperation)

- Wie konnten Sie sich auf ein gemeinsames Ziel einigen? Wie haben Sie ein gemeinsames Ziel gefunden?

(Tausch, Kompromiss, Integrativer Konsens)

Würden sie die Zusammenarbeit mit ? (Institution xy) als Kooperation bezeichnen? $(\rightarrow$ Allgemeine Definition:

Kooperation beinhaltet strukturelle, prozessuale und ergebnisbezogene Aspekte. Von kooperativem Handeln wird gesprochen, wenn sich die Kooperationspartner als wechselseitig gleichberechtigt ansehen, sie dialogisch kommunizieren und das Ergebnis eine gemeinsame Entscheidung ist, die von allen Beteiligten anerkannt wird)

Warum haben sie sich in diesem Falle für eine Kooperation entschieden?

Warum kooperieren sie mit anderen Institutionen?

Warum haben sie sich für die Strategie der Kooperation entschieden?

Wie würden sie die Interessen der Akteure bzw. Kooperationspartner beschreiben?

Halten sie diese Zusammenarbeit für innovativ?

Ist diese Zusammenarbeit eine Innovation für ihr Amt?

Werden durch die Zusammenarbeit innovative Lösungen umgesetzt?

Welchen Nutzen hat ihr Amt von der Zusammenarbeit mit anderen Organisationen?

Welchen Nutzen versprechen sie sich von der Zusammenarbeit?

Integration:

Für welche Personengruppen ist der Aufenthalt im Wald am wichtigsten?

Für welche Personengruppen kann eine integrative Maßnahme am wichtigsten sein?

- Setzt sich ihr Amt für die Interessen dieser Gruppen ein?

- Sind Sie der Meinung Problemgruppen sollten den Wald nutzen?

- Kann der Wald eine positive Wirkung auch für Problemgruppen haben?

- Wie würden Sie die Nutzung des Waldes durch Problemgruppen charakterisieren?

- Welche Auswirkungen hat die Nutzung des Waldes durch diese Gruppen?

- Wie werden schwierige Personengruppen im Wald behandelt? Wie wird mit innen umgegangen? 
Schlussfragen:

Position des Amtes:

Können Ihrer Meinung nach Konflikte zwischen sozialen Randgruppen und anderen Interessensgruppen an den Grünflächen identifiziert werden?

- Um welche anderen Interessensgruppen handelt es sich dabei?

- Wie verhalten sie sich als Behörde bzw. Amt in diesen Situationen

(Vermittler, Konfliktlöser, Konfliktpartner)?

\section{Kategoriensystem und Definitionen}

\section{Deskription}

\begin{tabular}{|c|c|c|c|c|c|}
\hline Kategorie & Code 1. Ebene & $\begin{array}{l}\text { Code } 2 . \\
\text { Ebene }\end{array}$ & $\begin{array}{l}\text { Code } 3 . \\
\text { Ebene }\end{array}$ & Code 4 Ebene & $\begin{array}{c}\text { Definition und } \\
\text { Beispiele }\end{array}$ \\
\hline \multirow[t]{9}{*}{$\begin{array}{ll}\text { Kontakte } \\
\text { Verhältnis }\end{array}$} & & & & & $\begin{array}{l}\text { Art des Kontaktes } \\
\text { und Verhältnisses }\end{array}$ \\
\hline & Berührungspunkte & & & & $\begin{array}{l}\text { Wer nimmt den } \\
\text { Kontakt auf (z.B. } \\
\text { Gruppe, } \\
\text { Institution); aus } \\
\text { welchen Gründen } \\
\text { (z.B. Anlaufstelle) }\end{array}$ \\
\hline & & Konflikt & & & $\begin{array}{lr}\text { Konflikt } & \text { als } \\
\text { Auslöser für den } \\
\text { Kontakt }\end{array}$ \\
\hline & & & $\begin{array}{l}\text { Art des } \\
\text { Konfliktes }\end{array}$ & & $\begin{array}{l}\text { Beschreibung des } \\
\text { Konflikts }\end{array}$ \\
\hline & & & & Interessenskonflikt & $\begin{array}{l}\text { Unterschiedliche } \\
\text { Interessen treffen } \\
\text { aufeinander z.B. } \\
\text { in der Nutzung }\end{array}$ \\
\hline & & & & $\begin{array}{ll}\text { Aufenthalt an } \\
\text { Orten }\end{array}$ & $\begin{array}{l}\text { Der Aufenthalt an } \\
\text { bestimmten Orten } \\
\text { ist Auslöser des } \\
\text { Konflikts, z.B. an } \\
\text { öffentlichen } \\
\text { Plätzen, } \\
\text { Grünflächen, im } \\
\text { Wald }\end{array}$ \\
\hline & & & & $\begin{array}{l}\text { Umgang } \\
\text { miteinander }\end{array}$ & $\begin{array}{l}\text { Der Umgang } \\
\text { zwischen } \\
\text { Interessenparteien } \\
\text { führt zum Konflikt, } \\
\text { z.B. durch } \\
\text { rücksichtsloses } \\
\text { Verhalten }\end{array}$ \\
\hline & & & & Lärm & $\begin{array}{ll}\text { Lärmen } & \text { einer } \\
\text { Gruppen } & \text { führt } \\
\text { zum Konflikt } & \\
\end{array}$ \\
\hline & & & & $\begin{array}{l}\text { Nachbarschaftsver- } \\
\text { hältnis }\end{array}$ & $\begin{array}{l}\text { Das Verhalten } \\
\text { einer Gruppe stört } \\
\text { die Menschen im } \\
\text { direkten Umfeld }\end{array}$ \\
\hline
\end{tabular}




\begin{tabular}{|l|l|l|l|l|l|}
\hline Kategorie & Code 1. Ebene & $\begin{array}{c}\text { Code 2. } \\
\text { Ebene }\end{array}$ & $\begin{array}{c}\text { Code 3. } \\
\text { Ebene }\end{array}$ & \multicolumn{1}{|c|}{ Code 4 Ebene } & $\begin{array}{l}\text { Definition und } \\
\text { Beispiele }\end{array}$ \\
\hline & & & & $\begin{array}{l}\text { Einhaltung von } \\
\text { Regeln }\end{array}$ & $\begin{array}{l}\text { Vorgegebene } \\
\text { Regeln werden } \\
\text { nicht eingehalten } \\
\text { und ein Konflikt } \\
\text { entsteht }\end{array}$ \\
\hline & & & Kommunikation & $\begin{array}{l}\text { Die Art der } \\
\text { Kommunikation } \\
\text { führt zum Konflikt }\end{array}$ \\
\hline & & & Müll & $\begin{array}{l}\text { Das Hinterlassen } \\
\text { von Müll führt zum } \\
\text { Konflikt mit } \\
\text { Gruppen }\end{array}$ \\
\hline & & & $\begin{array}{l}\text { Durch das } \\
\text { Mitführen von } \\
\text { Hunden treten } \\
\text { Konflikte auf }\end{array}$ \\
\hline & & & $\begin{array}{l}\text { Sonstige Günde, } \\
\text { die zu Konflikten } \\
\text { mit } \\
\text { Personengruppen } \\
\text { führen }\end{array}$ \\
\hline & Charakterisierung & & $\begin{array}{l}\text { Beschreibungen } \\
\text { und } \\
\text { Eigenschaften der } \\
\text { Gruppen, mit } \\
\text { denen Kontakt } \\
\text { besteht }\end{array}$ \\
\hline & & & & \\
\hline
\end{tabular}

\section{Hypothese I}

\begin{tabular}{|c|c|c|c|c|c|}
\hline Kategorie & Code 1. Ebene & Code 2. Ebene & $\begin{array}{l}\text { Code } 3 \\
\text { Ebene }\end{array}$ & $\begin{array}{l}\text { Code } 4 . \\
\text { Ebene }\end{array}$ & $\begin{array}{c}\text { Definition und } \\
\text { Beispiele }\end{array}$ \\
\hline \multirow[t]{7}{*}{ 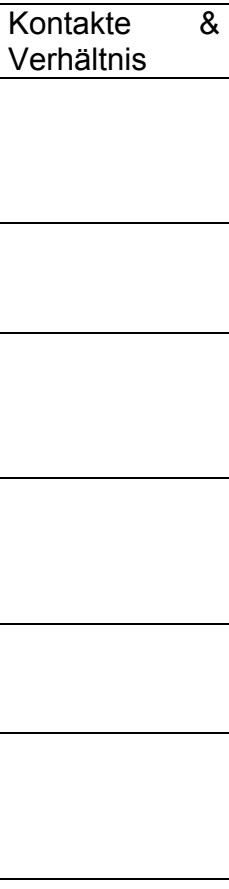 } & & & & & s.o. \\
\hline & $\begin{array}{l}\text { Problem- bzw. } \\
\text { Konfliktlösung }\end{array}$ & & & & $\begin{array}{l}\text { Mit welchen } \\
\text { Instrumenten wird } \\
\text { ein Problem oder } \\
\text { ein Konflikt gelöst }\end{array}$ \\
\hline & & Kooperation & & & $\begin{array}{l}\text { Das Problem wird } \\
\text { durch Kooperation } \\
\text { gelöst }\end{array}$ \\
\hline & & Runde Tische & & & $\begin{array}{l}\text { Das Problem wird } \\
\text { durch die Arbeit an } \\
\text { „Runden Tischen“ } \\
\text { gelöst }\end{array}$ \\
\hline & & $\begin{array}{ll}\text { Angebot } & \text { für } \\
\text { Randgruppen } & \end{array}$ & & & $\begin{array}{l}\text { Das Problem wird } \\
\text { durch ein Angebot } \\
\text { für die Gruppen } \\
\text { gelöst }\end{array}$ \\
\hline & & Finanzielle Mittel & & & $\begin{array}{l}\text { Das Problem wird } \\
\text { durch die Zahlung } \\
\text { von Geld gelöst }\end{array}$ \\
\hline & & Information & & & $\begin{array}{lr}\text { Das } & \text { Problem wird } \\
\text { durch } & \text { Informationen } \\
\text { zur } & \text { Aufklärung } \\
\text { gelöst } & \end{array}$ \\
\hline & & $\begin{array}{l}\text { Gespräche } \\
\text { Kommunikation }\end{array}$ & & & $\begin{array}{l}\text { Das Problem wird } \\
\text { durch } \\
\text { kommunikative } \\
\text { Instrumente gelöst }\end{array}$ \\
\hline
\end{tabular}




\begin{tabular}{|c|c|c|c|c|c|}
\hline Kategorie & Code 1. Ebene & Code 2. Ebene & $\begin{array}{l}\text { Code } 3 \\
\text { Ebene }\end{array}$ & $\begin{array}{c}\text { Code } 4 . \\
\text { Ebene }\end{array}$ & $\begin{array}{c}\text { Definition und } \\
\text { Beispiele }\end{array}$ \\
\hline & & Sonstiges & & & $\begin{array}{l}\text { Sonstige } \\
\text { Instrumente, die zur } \\
\text { Lösung des } \\
\text { Problems oder } \\
\text { Konflikts beitragen }\end{array}$ \\
\hline & $\begin{array}{l}\text { Ansprüche der } \\
\text { Randgruppen }\end{array}$ & & & & $\begin{array}{l}\text { Erläuterung der } \\
\text { Ansprüche der } \\
\text { Gruppen an Grün- } \\
\text { und Waldflächen }\end{array}$ \\
\hline & & Treffpunkt & & & $\begin{array}{l}\text { Anspruch an einen } \\
\text { Ort um sich zu } \\
\text { treffen }\end{array}$ \\
\hline & & $\begin{array}{l}\text { Wohnraumer- } \\
\text { weiterung }\end{array}$ & & & $\begin{array}{lr}\text { Orte um sich } \\
\text { außerhalb } & \text { der } \\
\text { Wohnung } & \\
\text { aufzuhalten } & \end{array}$ \\
\hline & & Soziales Umfeld & & & $\begin{array}{l}\text { Orte als } \\
\text { Interaktionsraum }\end{array}$ \\
\hline & & Aggressionsabbau & & & $\begin{array}{l}\text { Orte an denen } \\
\text { Aggressionen } \\
\text { abgebaut werden } \\
\text { können }\end{array}$ \\
\hline & & Freiraum & & & $\begin{array}{l}\text { Orte, die Freiraum } \\
\text { für eigenes Tun } \\
\text { schaffen }\end{array}$ \\
\hline & & $\begin{array}{l}\text { Eigenverantwort- } \\
\text { lichkeit }\end{array}$ & & & $\begin{array}{l}\text { Orte an denen } \\
\text { eigenverantwortlich, } \\
\text { ohne direkte } \\
\text { Kontrolle gehandelt } \\
\text { werden kann }\end{array}$ \\
\hline & & Spielmöglichkeiten & & & $\begin{array}{l}\text { Orte an } \begin{array}{l}\text { denen } \\
\text { gespielt } \\
\text { kann }\end{array}\end{array}$ \\
\hline & & $\begin{array}{l}\text { Ästhetische } \\
\text { Aspekte }\end{array}$ & & & $\begin{array}{l}\text { Orte die wegen ihrer } \\
\text { Schönheit und } \\
\text { Eigenarten } \\
\text { aufgesucht werden }\end{array}$ \\
\hline & & Sonstiges & & & $\begin{array}{l}\text { Sonstige Ansprüche } \\
\text { der Gruppen an } \\
\text { Grün- } \\
\text { Waldflächen }\end{array}$ \\
\hline & $\begin{array}{l}\text { Erfüllung der } \\
\text { Ansprüche }\end{array}$ & & & & $\begin{array}{l}\text { Werden diese } \\
\text { Ansprüche von } \\
\text { Institutionen erüllt }\end{array}$ \\
\hline Maßnahmen & & & & & $\begin{array}{lc}\text { Projekte } & \text { und } \\
\text { Maßnahmen } & \text { zur } \\
\text { Integration } & \\
\end{array}$ \\
\hline & $\begin{array}{l}\text { Finden } \\
\text { Maßnahmen zur } \\
\text { Integration statt }\end{array}$ & & & & \begin{tabular}{ll}
\multicolumn{2}{l}{ Maßnahmen, die im } \\
Wald oder auf \\
Grünflächen mit \\
dem Ziel der \\
Integration \\
stattfinden
\end{tabular} \\
\hline & & ja & & & $\begin{array}{l}\text { Es finden Projekte } \\
\text { statt }\end{array}$ \\
\hline & & nein & & & $\begin{array}{ll}\text { Es finden } & \text { keine } \\
\text { Projekte statt } & \\
\end{array}$ \\
\hline & $\begin{array}{l}\text { Beschreibung der } \\
\text { Maßnahme }\end{array}$ & & & & $\begin{array}{ll}\text { Genaue } & \\
\text { Beschreibung } & \text { des } \\
\text { Ablaufes } & \text { und } \\
\text { Inhaltes } & \text { der } \\
\text { Maßnahme } & \\
\end{array}$ \\
\hline & Partner & & & & $\begin{array}{l}\text { An den Projekten } \\
\text { beteiligte Personen } \\
\text { und Institutionen }\end{array}$ \\
\hline
\end{tabular}




\begin{tabular}{|c|c|c|c|c|c|}
\hline Kategorie & Code 1. Ebene & Code 2. Ebene & $\begin{array}{l}\text { Code } 3 \\
\text { Ebene }\end{array}$ & $\begin{array}{c}\text { Code } 4 . \\
\text { Ebene }\end{array}$ & $\begin{array}{c}\text { Definition und } \\
\text { Beispiele }\end{array}$ \\
\hline & Personengruppen & & & & $\begin{array}{ll}\text { Zielgruppe } & \text { der } \\
\text { Projekte } & \text { und } \\
\text { Maßnahmen } & \\
\end{array}$ \\
\hline & & Jugendliche & & & $\begin{array}{l}\text { Schwererziehbare } \\
\text { und ausländische } \\
\text { Jugendliche }\end{array}$ \\
\hline & & Migraten & & & $\begin{array}{l}\text { Menschen mit } \\
\text { Migrtionshintergrund }\end{array}$ \\
\hline & & Ausländer & & & $\begin{array}{l}\text { Personen mit } \\
\text { ausländischer } \\
\text { Herkunft }\end{array}$ \\
\hline & & Langzeitarbeitslose & & & $\begin{array}{l}\text { Personen, die sich } \\
\text { seit einem Jahr und } \\
\text { länger nicht in Arbeit } \\
\text { befinden }\end{array}$ \\
\hline & & Behinderte & & & $\begin{array}{l}\text { Menschen, die } \\
\text { körperlichen und } \\
\text { geistigen } \\
\text { Einschränkungen } \\
\text { leben }\end{array}$ \\
\hline & & Suchtkranke & & & \begin{tabular}{lr} 
Menschen, & die \\
psychisch & und \\
körperlich & von \\
Substanzen & \\
abhängig sind, & z.B. \\
\multicolumn{2}{l}{ Alkohol, Drogen }
\end{tabular} \\
\hline & & Straffällige & & & $\begin{array}{l}\text { Menschen, die eine } \\
\text { Strafe ableisten, } \\
\text { abgeleistet haben } \\
\text { oder diese noch } \\
\text { ableisten müssen }\end{array}$ \\
\hline & & Wohnungslose & & & $\begin{array}{l}\text { Menschen, die } \\
\text { keinen festen } \\
\text { Wohnsitz haben }\end{array}$ \\
\hline & & Sonstige & & & $\begin{array}{ll}\text { Sonstige } & \\
\text { Zielgruppen } & \text { der } \\
\text { Projekte } & \text { und } \\
\text { Maßnahmen } & \\
\end{array}$ \\
\hline & $\begin{array}{ll}\text { Dauer } & \text { der } \\
\text { Maßnahmen }\end{array}$ & & & & $\begin{array}{l}\text { Zeitlicher Umfang } \\
\text { der Projekte und } \\
\text { Maßnahmen }\end{array}$ \\
\hline & & Kurzfristig & & & $\begin{array}{l}\text { Tage, Wochen, 1-6 } \\
\text { Monate }\end{array}$ \\
\hline & & Langfristig & & & $1 \mathrm{Jahr}$ und mehr \\
\hline & & Sonstiges & & & $\begin{array}{l}\text { Weitere Angaben } \\
\text { zur Dauer der } \\
\text { Projekte } \\
\text { Maßnahmen }\end{array}$ \\
\hline Integration & & & & & $\begin{array}{l}\text { Ziel der Projekte } \\
\text { und Maßnahmen }\end{array}$ \\
\hline & Integrativer Erfolg & & & & $\begin{array}{l}\text { Auswirkungen, } \\
\text { Effekte der } \\
\text { Maßnahmen auf die } \\
\text { Personengruppen }\end{array}$ \\
\hline & $\begin{array}{l}\text { Äußerungen } \mathrm{zu} \\
\text { den } \\
\text { Personengruppen }\end{array}$ & & & & $\begin{array}{ll}\text { Ablehnende } & \text { oder } \\
\text { respektvolle } & \\
\text { Äußerungen zu den } \\
\text { Personen } \\
\text { Seiten } \\
\text { Institutionen }\end{array}$ \\
\hline & & Aufenthalt im Wald & & & $\begin{array}{l}\text { Bewertung des } \\
\text { Aufenthalts im Wald } \\
\text { (ablehnend oder } \\
\text { tolerierend) }\end{array}$ \\
\hline
\end{tabular}




\begin{tabular}{|c|c|c|c|c|c|}
\hline Kategorie & Code 1. Ebene & Code 2. Ebene & $\begin{array}{l}\text { Code } 3 \\
\text { Ebene }\end{array}$ & $\begin{array}{c}\text { Code } 4 . \\
\text { Ebene }\end{array}$ & $\begin{array}{l}\text { Definition und } \\
\text { Beispiele }\end{array}$ \\
\hline & & $\begin{array}{l}\text { Integrative } \\
\text { Maßnahme }\end{array}$ & & & $\begin{array}{l}\text { Äußerungen der } \\
\text { Institutionen ob } \\
\text { integrative } \\
\text { Maßnahmen } \\
\text { sinnvoll oder sinnlos } \\
\text { sind }\end{array}$ \\
\hline & $\begin{array}{l}\text { Haltung des } \\
\text { Amtes }\end{array}$ & & & & $\begin{array}{ll}\text { Meinungen, } & \\
\text { Aussagen } & \text { und } \\
\text { Bewertungen } & \text { der } \\
\text { Institutionen } & \end{array}$ \\
\hline & & $\begin{array}{ll}\text { Einsetzen für } \\
\text { Randgruppen }\end{array}$ & & & $\begin{array}{l}\text { Institution kümmern } \\
\text { sich um } \\
\text { Problemgruppen }\end{array}$ \\
\hline & & $\begin{array}{l}\text { Nutzung des } \\
\text { Waldes / Grüns }\end{array}$ & & & $\begin{array}{ll}\text { Äußerungen } & \text { und } \\
\text { Haltung } & \text { der } \\
\text { Institution } & \text { zur } \\
\text { Nutzung } & \\
\end{array}$ \\
\hline & & $\begin{array}{l}\text { Positive Wirkung } \\
\text { des Waldes / } \\
\text { Grüns }\end{array}$ & & & $\begin{array}{ll}\text { Äußerungen der } \\
\text { Institutionen zur } \\
\text { Wirkung auf die } \\
\text { Problemgruppen }\end{array}$ \\
\hline & & $\begin{array}{l}\text { Charakterisierung } \\
\text { der Nutzung durch } \\
\text { Randgruppen }\end{array}$ & & & $\begin{array}{ll}\text { Bewertung } & \text { und } \\
\text { Äußerungen } & \text { der } \\
\text { Nutzung durch } & \text { die } \\
\text { Randgruppen } & \\
\end{array}$ \\
\hline & & $\begin{array}{l}\text { Auswirkung der } \\
\text { Nutzung }\end{array}$ & & & $\begin{array}{lr}\text { Bewertung } & \text { der } \\
\text { Auswirkung } & \text { durch } \\
\text { die Institution } & \\
\end{array}$ \\
\hline & & $\begin{array}{l}\text { Umgang mit den } \\
\text { Personengruppen }\end{array}$ & & & $\begin{array}{l}\text { Verhaltensweisen } \\
\text { der Institution } \\
\text { gegenüber den } \\
\text { Randgruppen }\end{array}$ \\
\hline & $\begin{array}{l}\text { Position des } \\
\text { Amtes }\end{array}$ & & & & $\begin{array}{lr}\text { Allgemeine } & \\
\text { Einstellungen } & \text { und } \\
\text { Meinungen } & \text { der } \\
\text { Institution } & \text { zu } \\
\text { Projekten } & \text { für } \\
\text { Problemgruppen } & \\
\end{array}$ \\
\hline & & Vermittler & & & $\begin{array}{l}\text { Vermittlerposition } \\
\text { der Institution im } \\
\text { Konfliktfall }\end{array}$ \\
\hline & & Konfliktlöser & & & $\begin{array}{l}\text { Institution tritt als } \\
\text { Löser des Problems } \\
\text { auf }\end{array}$ \\
\hline & & Konfliktpartner & & & $\begin{array}{l}\text { Institution ist direkt } \\
\text { in den Konflikt mit } \\
\text { Problemgruppen } \\
\text { involviert }\end{array}$ \\
\hline
\end{tabular}




\section{Hypothese II}

\begin{tabular}{|c|c|c|c|c|c|}
\hline Kategorie & Code 1. Ebene & Code 2. Ebene & $\begin{array}{l}\text { Code } 3 . \\
\text { Ebene }\end{array}$ & $\begin{array}{c}\text { Code } 4 . \\
\text { Ebene }\end{array}$ & $\begin{array}{l}\text { Definition und } \\
\text { Beispiele }\end{array}$ \\
\hline \multirow[t]{16}{*}{ Kooperation } & & & & & $\begin{array}{l}\text { Allgemeine Aussage } \\
\text { über } \\
\text { Zusammenarbeit }\end{array}$ \\
\hline & Struktur & & & & $\begin{array}{ll}\text { Aussagen über } & \text { die } \\
\text { Struktur } & \text { der } \\
\text { Kooperation } & \\
\end{array}$ \\
\hline & & $\begin{array}{l}\text { Wechselseitige } \\
\text { Gleichberechtigung }\end{array}$ & & & $\begin{array}{l}\text { Kooperationspartner } \\
\text { sind gleichberechtigt }\end{array}$ \\
\hline & & $\begin{array}{l}\text { Gleiche Verfahrens- } \\
\text { und } \\
\text { Entscheidungsrechte }\end{array}$ & & & $\begin{array}{lr}\text { Partner } & \text { haben } \\
\text { identisch großen } \\
\text { Einfluss auf das } \\
\text { Verfahren und die } \\
\text { Entscheidung }\end{array}$ \\
\hline & & $\begin{array}{l}\text { Gleiche Rechte und } \\
\text { Pflichten der Partner }\end{array}$ & & & $\begin{array}{l}\text { Partner sind im } \\
\text { Kooperationsprozess } \\
\text { mit gleichen Rechten } \\
\text { und Pflichten } \\
\text { ausgestattet }\end{array}$ \\
\hline & & $\begin{array}{l}\text { Einseitige } \\
\text { Handlungsmöglichkeit }\end{array}$ & & & $\begin{array}{lr}\text { Nur ein } & \text { Partner } \\
\text { besitzt } & \text { durch } \\
\text { Ressourcen } & \text { die } \\
\text { Möglichkeit } & \text { zu } \\
\text { handeln, } & \text { z.B. } \\
\text { Kenntnis, Information }\end{array}$ \\
\hline & & $\begin{array}{l}\text { Einseitige } \\
\text { Entscheidungsmacht }\end{array}$ & & & $\begin{array}{lr}\text { Nur ein } & \text { Partner } \\
\text { besitzt } & \text { durch } \\
\text { Ressourcen } & \text { die } \\
\text { Möglichkeit } & \text { zu } \\
\text { entscheiden, z.B. } & \text { Kenntnis, Information, } \\
\text { finanzielle Mittel }\end{array}$ \\
\hline & & $\begin{array}{l}\text { Gesetzliche } \\
\text { Regelungen }\end{array}$ & & & $\begin{array}{l}\text { Verordnungen und } \\
\text { Gesetze geben den } \\
\text { Handlungsrahmen } \\
\text { der Partner vor }\end{array}$ \\
\hline & Prozess & & & & $\begin{array}{ll}\text { Aussagen über } & \text { den } \\
\text { Prozess } & \text { der } \\
\text { Kooperation } & \end{array}$ \\
\hline & & $\begin{array}{l}\text { Dialogische } \\
\text { Kommunikation }\end{array}$ & & & $\begin{array}{l}\text { Kommunikation läuft } \\
\text { wechselseitig ab }\end{array}$ \\
\hline & & $\begin{array}{l}\text { "face-to-face" } \\
\text { Kontakte }\end{array}$ & & & $\begin{array}{l}\text { Direkter Kontakt der } \\
\text { Partner durch Treffen }\end{array}$ \\
\hline & & Abstimmung & & & $\begin{array}{lr}\text { Interessen, Ziel } & \text { und } \\
\text { Werte } & \text { der } \\
\text { Kooperation } & \text { und } \\
\text { Partner } & \text { werden } \\
\text { miteinander } & \\
\text { abgestimmt } & \end{array}$ \\
\hline & Ergebnis & $\begin{array}{l}\text { Ziel ist die von Allen } \\
\text { akzeptierte Lösung }\end{array}$ & & & $\begin{array}{l}\text { Partner akzeptieren } \\
\text { die Lösung des } \\
\text { Problems }\end{array}$ \\
\hline & & Freiwillige Einigung & & & $\begin{array}{l}\text { Partner einigen sich } \\
\text { freiwillig auf ein } \\
\text { Ergebnis }\end{array}$ \\
\hline & & $\begin{array}{l}\text { Konkrete } \\
\text { Problemlösung }\end{array}$ & & & $\begin{array}{l}\text { Das Ziel der Partner } \\
\text { ist eine konkrete } \\
\text { Lösung des Problems }\end{array}$ \\
\hline & & $\begin{array}{l}\text { gemeinsame } \\
\text { Entscheidung }\end{array}$ & & & $\begin{array}{l}\text { Die Lösung des } \\
\text { Problems ist eine } \\
\text { gemeinsame } \\
\text { Entscheidung der } \\
\text { Partner, die von allen } \\
\text { anerkannt wird }\end{array}$ \\
\hline
\end{tabular}




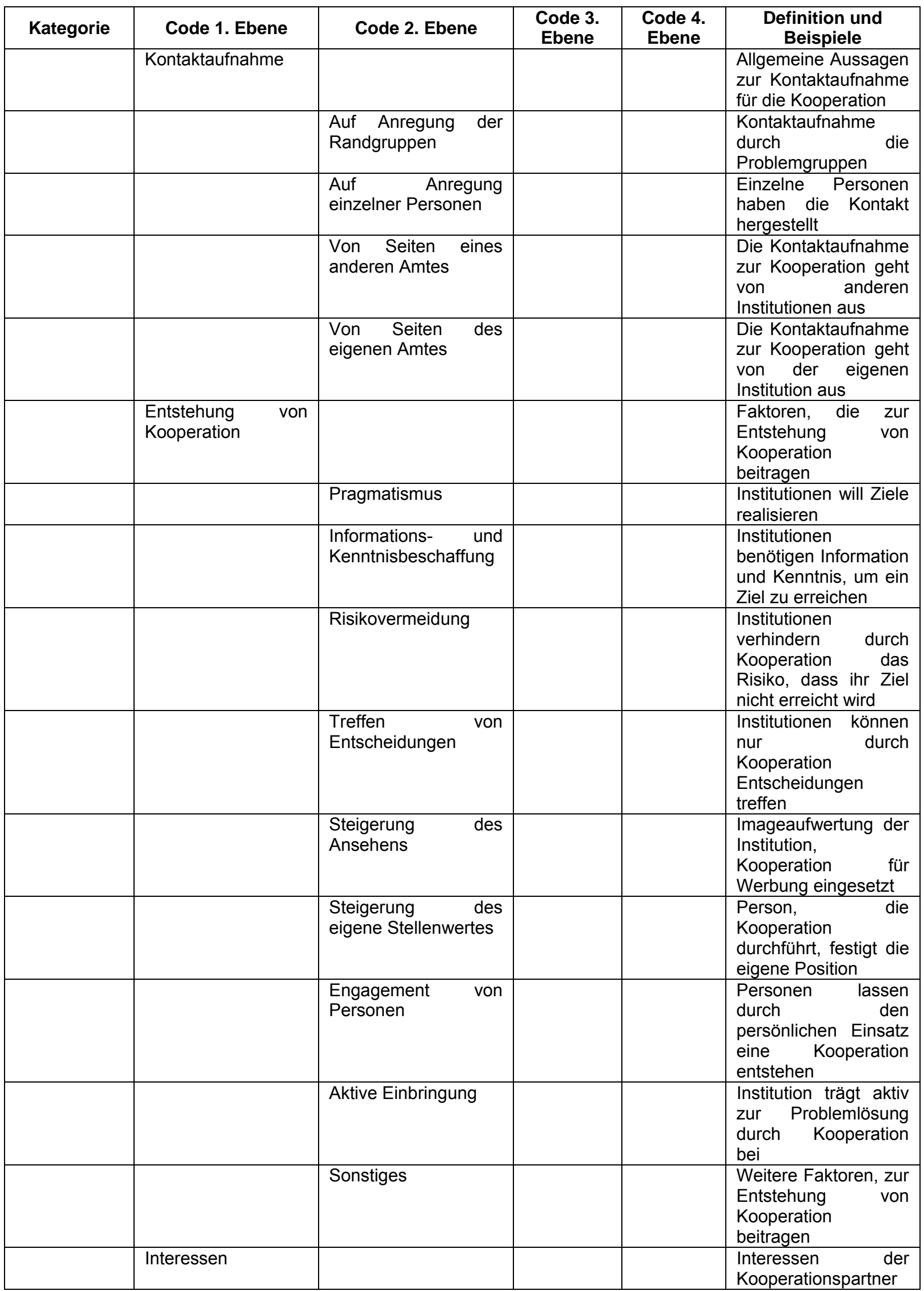




\begin{tabular}{|c|c|c|c|c|c|}
\hline Kategorie & Code 1. Ebene & Code 2. Ebene & $\begin{array}{l}\text { Code } 3 . \\
\text { Ebene }\end{array}$ & $\begin{array}{l}\text { Code } 4 . \\
\text { Ebene }\end{array}$ & $\begin{array}{c}\text { Definition und } \\
\text { Beispiele }\end{array}$ \\
\hline & & identisch & & & $\begin{array}{l}\text { Interessen, die } \\
\text { vollständig } \\
\text { miteinander vereinbar } \\
\text { sind }\end{array}$ \\
\hline & & komplementär & & & $\begin{array}{l}\text { Interessen, die in } \\
\text { eine Richtung wirken, } \\
\text { aber einen } \\
\text { unterschiedlichen } \\
\text { Bezugsgegenstand } \\
\text { besitzen }\end{array}$ \\
\hline & & konkurrierend & & & $\begin{array}{l}\text { Interessen, bei sich in } \\
\text { der Verfolgung } \\
\text { gegenseitig } \\
\text { behindern }\end{array}$ \\
\hline & & antagonistisch & & & $\begin{array}{l}\text { Interessen, die sich } \\
\text { gegenseitig } \\
\text { ausschließen }\end{array}$ \\
\hline & Verhandlungsmodus & & & & $\begin{array}{l}\text { Art und Weise der } \\
\text { Einigung auf ein Ziel } \\
\text { in der Kooperation }\end{array}$ \\
\hline & & Tausch & & & $\begin{array}{lr}\text { Partner } & \text { setzt } \\
\text { Interessen durch und } \\
\text { entschädigt } \\
\text { anderen Partner }\end{array}$ \\
\hline & & Kompromiss & & & $\begin{array}{l}\text { Partner verzichten } \\
\text { auf die vollständige } \\
\text { Verwirklichung ihrer } \\
\text { Ziele }\end{array}$ \\
\hline & & Integrativer Konsens & & & $\begin{array}{lr}\text { Partner finden } & \text { eine } \\
\text { Lösung des Problems } \\
\text { durch } & \text { das } \\
\text { gegenseitige } & \text { Lernen } \\
\text { voneinander } & \end{array}$ \\
\hline & Innovation & & & & 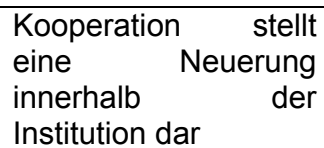 \\
\hline & & Merkmale & & & $\begin{array}{l}\text { Merkmale, die diese } \\
\text { Innovation } \\
\text { beschreiben }\end{array}$ \\
\hline & Nutzen & & & & $\begin{array}{l}\text { Allgemeine Aussagen } \\
\text { zum } \\
\text { Kooperationsnutzen }\end{array}$ \\
\hline & & $\begin{array}{ll}\text { Steigerung } & \text { der } \\
\text { eigenen Position }\end{array}$ & & & $\begin{array}{l}\text { Kooperation führt zur } \\
\text { erhöhtem Ansehen } \\
\text { und Image }\end{array}$ \\
\hline & & & des Amtes & & S.o. für die Institution \\
\hline & & & $\begin{array}{l}\text { des } \\
\text { Ausführ- } \\
\text { enden }\end{array}$ & & $\begin{array}{lcr}\text { S.o. für die } & \text { Person, } \\
\text { die für } & \text { die } \\
\text { Kooperation } & \\
\text { verantwortlich ist }\end{array}$ \\
\hline & & Informationsgewinn & & & $\begin{array}{l}\text { Kooperation liefert } \\
\text { Informationen über } \\
\text { bisher unbekannte } \\
\text { Bereiche }\end{array}$ \\
\hline & & $\begin{array}{lr}\text { Erweiterung des } & \text { des } \\
\text { Handlungsspielraums }\end{array}$ & & & $\begin{array}{l}\text { Institution erweitert } \\
\text { den } \\
\text { Zuständigkeitsbereich }\end{array}$ \\
\hline & & $\begin{array}{l}\text { Erschleißung } \\
\text { finanzieller } \\
\text { Ressourcen }\end{array}$ & & & $\begin{array}{l}\text { Institution erhält } \\
\text { durch Kooperation } \\
\text { finanzielle } \\
\text { Zuwendungen }\end{array}$ \\
\hline & & $\begin{array}{l}\text { Einflusssteigerung in } \\
\text { gesellschaftliche } \\
\text { Problembereiche }\end{array}$ & & & $\begin{array}{l}\text { Institution trägt zur } \\
\text { Lösung sozialer } \\
\text { Probleme bei }\end{array}$ \\
\hline
\end{tabular}




\begin{tabular}{|c|c|c|c|c|c|}
\hline Kategorie & Code 1. Ebene & Code 2. Ebene & $\begin{array}{c}\text { Code } 3 . \\
\text { Ebene }\end{array}$ & $\begin{array}{l}\text { Code } 4 . \\
\text { Ebene }\end{array}$ & $\begin{array}{c}\text { Definition und } \\
\text { Beispiele }\end{array}$ \\
\hline & & $\begin{array}{l}\text { Kontakt für weitere } \\
\text { Projekte }\end{array}$ & & & $\begin{array}{lr}\text { Institutionen nutzen } \\
\text { den Kontakt für neue } \\
\text { Projekte } \\
\text { Maßnahmen }\end{array}$ \\
\hline & & Synergieeffekt & & & $\begin{array}{l}\text { Vorteile, die für die } \\
\text { Partner durch die } \\
\text { Kooperation } \\
\text { entstehen }\end{array}$ \\
\hline & & Lerneffekt & & & $\begin{array}{lr}\text { Institutionen } & \text { lernen } \\
\text { durch } & \text { die } \\
\text { Kooperation, } & \text { z.B. } \\
\text { Aneignung } & \text { von } \\
\text { Qualifikationen } & \\
\end{array}$ \\
\hline & & Akzeptanz & & & 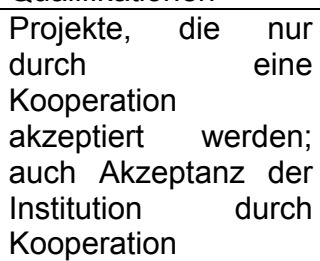 \\
\hline & & Sonstiges & & & $\begin{array}{l}\text { Sonstige Faktoren, } \\
\text { die den Nutzen der } \\
\text { Kooperation } \\
\text { beschreiben }\end{array}$ \\
\hline
\end{tabular}

\section{Hypothese III}

\begin{tabular}{|c|c|c|c|c|c|}
\hline Kategorie & Code 1. Ebene & Code 2. Ebene & $\begin{array}{c}\text { Code } 3 . \\
\text { Ebene }\end{array}$ & $\begin{array}{l}\text { Code } 4 . \\
\text { Ebene }\end{array}$ & Definition und Beispiele \\
\hline \multirow{9}{*}{ Maßnahmen } & & & & & S.o. \\
\hline & $\begin{array}{l}\text { Finanzielle } \\
\text { Ausstattung }\end{array}$ & & & & $\begin{array}{l}\text { Angaben zur Finanzierung } \\
\text { der Projekte und } \\
\text { Maßnahmen }\end{array}$ \\
\hline & & Von wem finanziert & & & $\begin{array}{l}\text { Institutionen, Investoren, } \\
\text { öffentliche Budgets, } \\
\text { Finanzierungswege der } \\
\text { Projekte }\end{array}$ \\
\hline & & Entschädigung & & & $\begin{array}{l}\text { Augleichszahlungen für die } \\
\text { Maßnahmen }\end{array}$ \\
\hline & & Maßnahmenförderung & & & $\begin{array}{lr}\text { Direkte Zahlungen für die } \\
\text { Maßnahmen, } & \text { keine } \\
\text { Übernahme } & \text { von } \\
\text { Personalkosten } & \\
\end{array}$ \\
\hline & & Sonstiges & & & $\begin{array}{l}\text { Weitere } \\
\text { Finanzierungsmöglichkeiten } \\
\text { der Maßnahmen und } \\
\text { Projekte }\end{array}$ \\
\hline & $\begin{array}{l}\text { Personelle } \\
\text { Ausstattung }\end{array}$ & & & & $\begin{array}{l}\text { Angaben zur Personenzahl, } \\
\text { die für die } \\
\text { Maßnahmendurchführung } \\
\text { verantwortlich sind }\end{array}$ \\
\hline & $\begin{array}{l}\text { Verlagerung } \\
\text { des } \\
\text { Aufgabenfeldes }\end{array}$ & & & & $\begin{array}{l}\text { Institution nimmt Integration } \\
\text { in den eigenen } \\
\text { Aufgabenbereich auf (vor } \\
\text { allen Forstverwaltung } \\
\text { interessant) }\end{array}$ \\
\hline & & $\begin{array}{l}\text { Erschließung von } \\
\text { Ressourcen }\end{array}$ & & & $\begin{array}{l}\text { Durch Verlagerung werden } \\
\text { neue Finanzierungswege } \\
\text { für die Institution eröffnet }\end{array}$ \\
\hline
\end{tabular}




\begin{tabular}{|c|c|c|c|c|c|}
\hline Kategorie & Code 1. Ebene & Code 2. Ebene & $\begin{array}{c}\text { Code } 3 . \\
\text { Ebene }\end{array}$ & $\begin{array}{l}\text { Code } 4 . \\
\text { Ebene }\end{array}$ & Definition und Beispiele \\
\hline & & Aufgabenerweiterung & & & $\begin{array}{lrr}\text { Integration wird als neue } \\
\text { Dienstleistung in den } \\
\text { Aufgabenbereich } & \text { der } \\
\text { Forstverwaltung } & \\
\text { aufgenommen } & \\
\end{array}$ \\
\hline & & $\begin{array}{l}\text { Personelle } \\
\text { Veränderung }\end{array}$ & & & $\begin{array}{ll}\text { Die neue Dienstleistung } \\
\text { geht mit einer } \\
\text { Personalveränderung } \\
\begin{array}{l}\text { einher, } \\
\text { Neueinstellungen }\end{array} \\
\end{array}$ \\
\hline & $\begin{array}{l}\text { Zukünftiges } \\
\text { Aufgabengebiet }\end{array}$ & & & & $\begin{array}{l}\text { Integration stellt ein } \\
\text { zukünftiges Aufgabengebiet } \\
\text { dar }\end{array}$ \\
\hline & & $\begin{array}{l}\text { Aufnahme in das } \\
\text { Tätigkeitsfeld }\end{array}$ & & & $\begin{array}{l}\text { Integration wird zukünftig } \\
\text { von der Forstverwaltung } \\
\text { angeboten }\end{array}$ \\
\hline & & Einmalige Aktion & & & $\begin{array}{l}\text { Integration wird von der } \\
\text { Forstverwaltung } \\
\text { zukünftig angeboten }\end{array}$ \\
\hline
\end{tabular}




\section{Institutionen der Interviewpartner}

\section{Göttingen:}

I1 Straßensozialarbeit

12 KiK e.V.

I3 Fachbereich Stadtgrün

14 Sozialplanung

15 Forstamt

16 Jugendamt

17 Arillus $\mathrm{GmbH}$

Kassel:

18 Sozialamt

19 Umwelt- und Gartenamt

110 Forstamt

111 Jugendamt

I12 Verwaltung staatliche Schlösser und Gärten

113 AGIL mbH

\section{Freiburg i.Br.:}

114 Jugendförderung

115 Forstamt

I16 Suchtbeauftragter der Stadt

117 Stadtrat

\section{Frankfurt a.M.:}

I18 Jugend- und Sozialamt „Ferienspiele“

I19 CeBeeF e.V.

120 Werkstatt Frankfurt e.V.

I21 Stadtgrün Fachbereich Forst

Hamburg:
122 passage $\mathrm{gGmbH}$
I23 Forstamt / Revierleitung

\section{Düsseldorf:}

I24 Garten-, Friedhofs- und Forstamt

Berlin:

I25 Landesforstamt

I26 Grünflächenamt (Berlin Mitte)

Nürnberg:

I27 mudra Waldprojekt 


\section{Eidesstattliche Erklärung}

Hiermit versichere ich gemäß $\S 4(2 e)$ der Promotionsordnung des Fachbereiches für Forstwissenschaften und Waldökologie der Georg-August-Universität Göttingen vom 08.01.1986, das ich die vorliegende Arbeit selbständig verfasst habe und keine anderen als die angegebenen Quellen und Hilfsmittel benutzt habe.

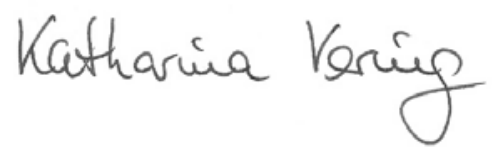

(Katharina Vering) 


\section{Curriculum vitae}

Name

Vering

Vorname

Katharina

Geburtstag

22.05.1977

Geburtsort

Rheda-Wiedenbrück, Nordrhein-Westfalen

Familienstand

ledig

Staatsangehörigkeit

deutsch

Berufliche Tätigkeiten

01/03 - 09/07 Promotion zum Thema „Integrationsprojekte als Aufgabe urbaner Forstwirtschaft - Ein Beitrag zur sozialen Nachhaltigkeit" (Rigorosum am 17.09.2007), gefördert von der GeorgLudwig-Hartig-Stiftung

seit 02 / 2006

Lehrerin an der Berufsbildenden Schule II in Northeim im Fach Politik in der Ausbildung zur/m Forstwirt/in (1. bis 3 . Ausbildungsjahr)

$11 / 01-12$ / 06

Wissenschaftliche Mitarbeiterin am Institut für Forstpolitik und Naturschutz der Georg-AugustUniversität Göttingen

\section{Studium}

10/96 - 11/01

Studium der Forstwissenschaft und Waldökologie an der Georg-August-Universität Göttingen,

Abschluss: Bachelor of Science (B.Sc.)

Studienschwerpunkt: Forstbetrieb und Waldnutzung Abschluss: Master of Science (M.Sc.)

\section{Schulausbildung}

$8 / 87-5 / 96$

Marienschule, Priv. Gymnasium f. Jungen u.

Mädchen -staatlich anerkannt- Lippstadt

Abschluss: allgemeine Hochschulreife

$8 / 83-7 / 87$

Brinkmannschule, Gemeinschaftsgrundschule, Langenberg

\section{Weiterführende Kenntnisse}

Sprachen:

Englisch (fließend in Wort und Schrift)

Software:

MS Office (Word, Excel, Power Point), SPSS, MAXQDA 